\title{
Energy Star Concepts for Highway Vehicles
}

\section{June 2003}

David L. Greene

Oak Ridge National Laboratory

Robert C. Gibson

The University of Tennessee

K. G. Duleep

Energy and Environmental Analysis, Inc. 


\title{
DOCUMENT AVAILABILITY
}

Reports produced after January 1,1996, are generally available free via the U.S. Department of Energy (DOE) Information Bridge:

Web site: http://www.osti.gov/bridge

Reports produced before January 1, 1996, may be purchased by members of the public from the following source:

\author{
National Technical Information Service \\ 5285 Port Royal Road \\ Springfield, VA 22161 \\ Telephone: 703-605-6000 (1-800-553-6847) \\ TDD: 703-487-4639 \\ Fax: 703-605-6900 \\ E-mail: info@ntis.fedworld.gov \\ Web site: http://www.ntis.gov/support/ordernowabout.htm
}

Reports are available to DOE employees, DOE contractors, Energy Technology Data Exchange (ETDE) representatives, and International Nuclear Information System (INIS) representatives from the following source:

Office of Scientific and Technical Information

P.O. Box 62

Oak Ridge, TN 37831

Telephone: 865-576-8401

Fax: 865-576-5728

E-mail: reports@adonis.osti.gov

Web site: http://www.osti.gov/contact.html

This report was prepared as an account of work sponsored by an agency of the United States Government. Neither the United States government nor any agency thereof, nor any of their employees, makes any warranty, express or implied, or assumes any legal liability or responsibility for the accuracy, completeness, or usefulness of any information, apparatus, product, or process disclosed, or represents that its use would not infringe privately owned rights. Reference herein to any specific commercial product, process, or service by trade name, trademark, manufacturer, or otherwise, does not necessarily constitute or imply its endorsement, recommendation, or favoring by the United States Government or any agency thereof. The views and opinions of authors expressed herein do not necessarily state or reflect those of the United States Government or any agency thereof. 


\title{
ENERGY STAR CONCEPTS FOR HIGHWAY VEHICLES
}

\author{
David L. Greene \\ Oak Ridge National Laboratory \\ Oak Ridge, Tennessee \\ Robert C. Gibson \\ The University of Tennessee \\ Knoxville, Tennessee \\ K. G. Duleep \\ Energy and Environmental Analysis, Inc. \\ Arlington, Virginia
}

June 2003

\author{
Prepared by \\ OAK RIDGE NATIONAL LABORATORY \\ P.O. Box 2008 \\ Oak Ridge, Tennessee 37831-6285 \\ managed by \\ UT-Battelle, LLC \\ for the \\ U.S. DEPARTMENT OF ENERGY \\ under contract DE-AC05-00OR22725
}





\section{TABLE OF CONTENTS}

Page

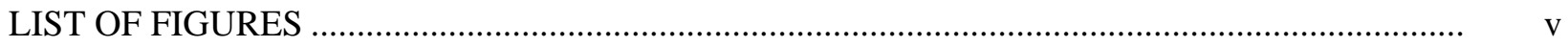

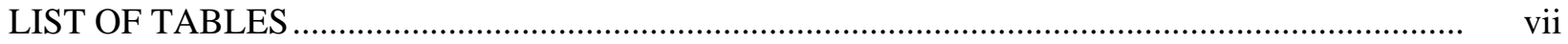

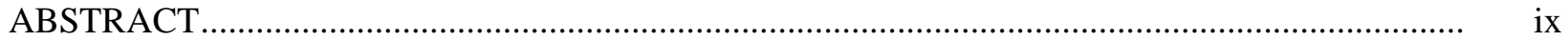

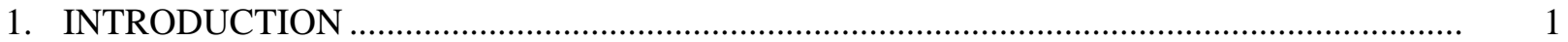

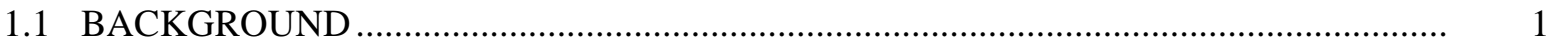

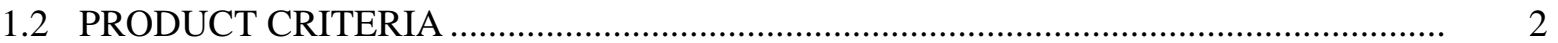

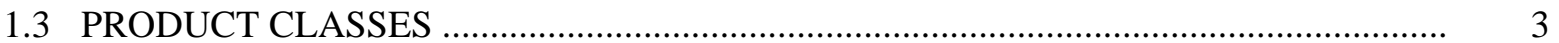

1.4 RAISING STANDARDS FOR EVOLVING TECHNOLOGY …............................... 3

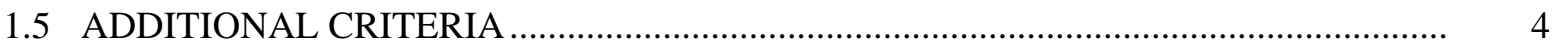

2. ENERGY STAR FOR HIGHWAY VEHICLES …............................................................

3. POTENTIAL ENERGY STAR METRICS FOR VEHICLES .............................................. 7

3.1 THE LIMITATIONS OF SIMPLE MPG ...........................................................................

3.2 ESTABLISHING ENERGY STAR METRICS FOR CARS AND LIGHT TRUCKS ....... 7

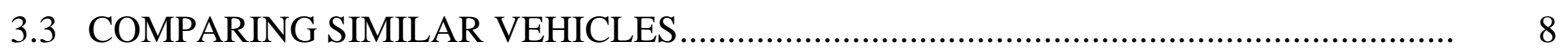

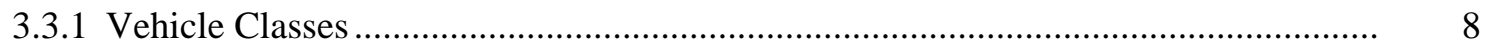

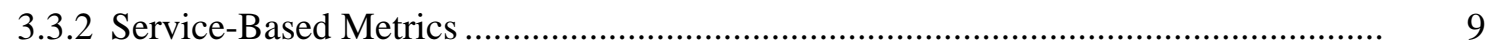

4. OUTCOME-BASED ENERGY STAR CRITERIA EXPLORED ….........................................

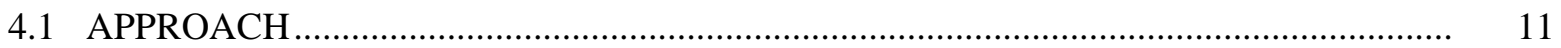

4.1.1 Method 1: Best Fuel Economy (MPG) ......................................................... 12

4.1.2 Method 2: Lowest Annual GHG Emissions ........................................................ 12

4.1.3 Method 3: Lowest Annual Oil Use ......................................................................... 13

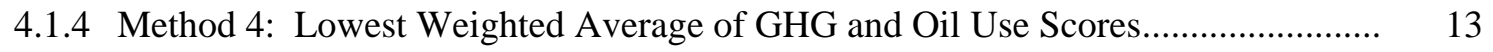

4.1.5 Method 5: Highest Footprint-MPG ..................................................................... 13

4.1.6 Methods 6 \& 7: Best Ratio of Actual to Predicted MPG Based on

Regression Analysis...................................................................................... 13

4.1.7 Method 8: Best Fuel Economy (MPG) in Each Body Type Class (Car vs. Truck) ... 14

4.1.8 Method 9: Best Fuel Economy (MPG) in Each Type-Size Class ............................ 14

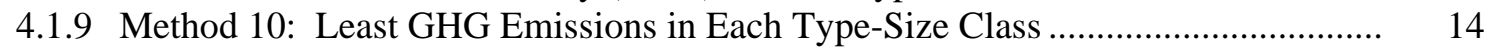

4.1.10 Method 11: Best Fuel Economy (MPG) in Each Market Class .................................. 14

4.1.11 Method 12: Lowest Ratio of Oil Use to Footprint ................................................... 14

4.1.12 Methods 13 \& 14: Best Ratio of GHG and Oil Use to Footprint .............................. 14

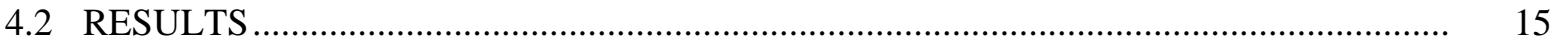

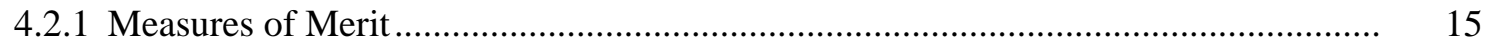

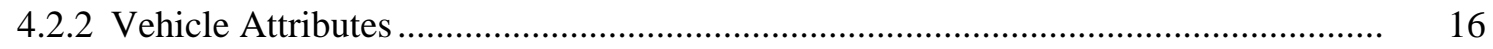

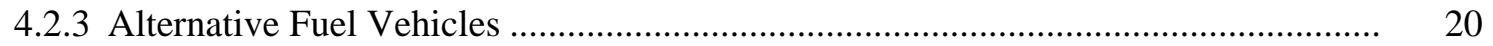

4.2.4 Strengths and Limitations of Each Method ............................................................. 20

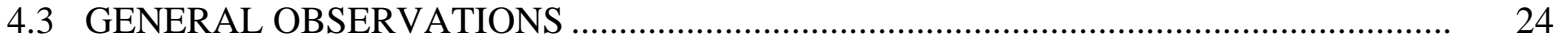

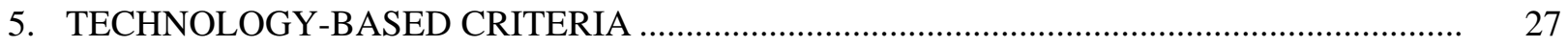

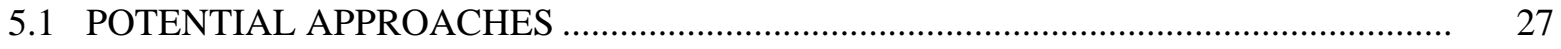

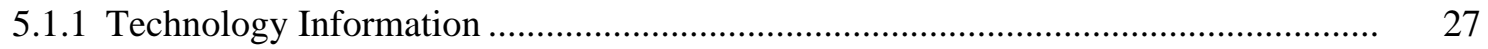

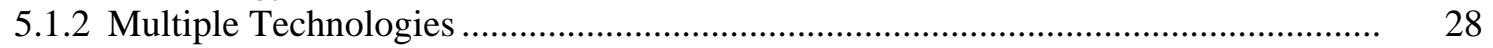




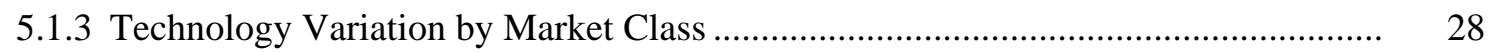

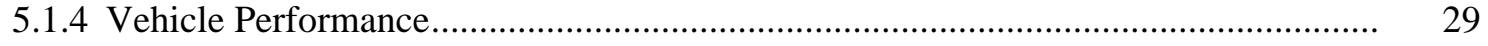

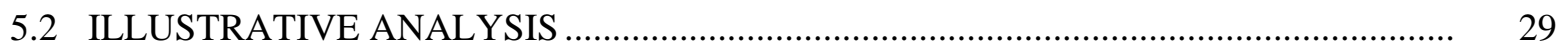

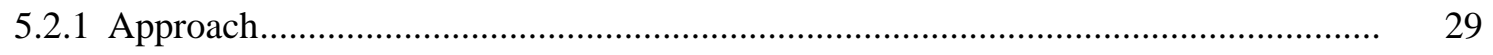

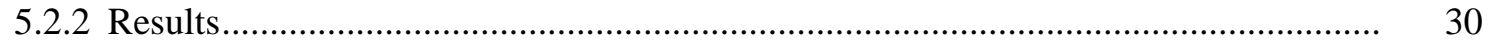

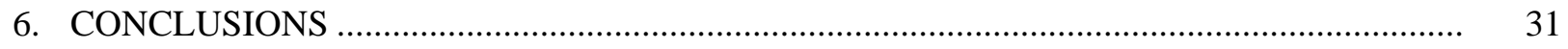

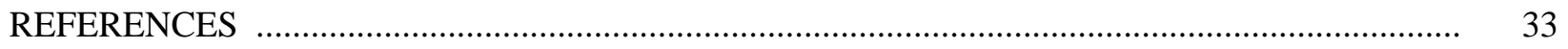

APPENDIX A. ALTERNATIVE EFFICIENCY-BASED APPROACH TO

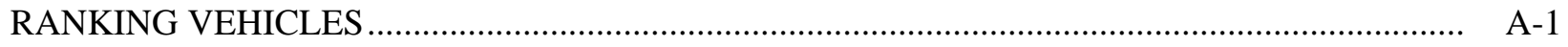

APPENDIX B. VEHICLES SELECTED BY EACH ENERGY STAR METHOD ........................ B-1 


\section{LIST OF FIGURES}

Figure $\quad$ Page

1 Percent Improvement in the Average MPG, GHG Emissions, and Oil Use Scores for Vehicles Selected by Each Energy Star Method.

2 Average Footprint for Vehicles Selected by Each Energy Star Method ............................... 18

3 Average Acceleration Time for Vehicles Selected by Each Energy Star Method ................. 18

4 Percentage of Vehicles Selected by Each Energy Star Method that are Trucks ................... 19

$5 \quad$ Number of Alternative Vehicles Selected by Each Energy Star Method.............................. 20 



\section{LIST OF TABLES}

\section{Table}

Page

$1 \quad$ Energy Star Criteria for Refrigerators by Class …............................................................

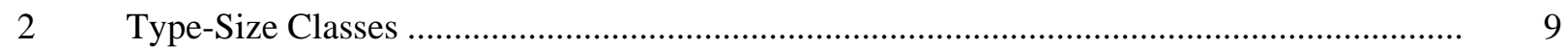

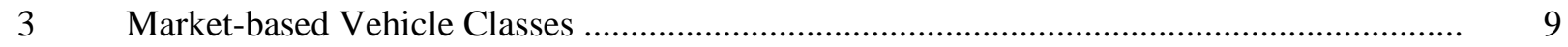

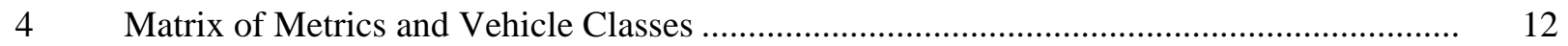

$5 \quad$ Average Measures of Merit for Vehicles Selected by Each Energy Star Rating Method...... 16

6 Average Attribute Scores for Vehicles Selected by Each Energy Star Rating Method ......... 17

$7 \quad$ Summary of Strengths and Limitations of Each Method ............................................... 24

$8 \quad$ Vehicle Technology Impacts and Data Availability ..................................................... 28 



\section{ABSTRACT}

The authors of this report, under the sponsorship of the U.S. Department of Energy (DOE) Office of Energy Efficiency and Renewable Energy (EERE) Weatherization and Intergovernmental Program, have investigated the possible application of Energy Star ratings to passenger cars and light trucks. This study establishes a framework for formulating and evaluating Energy Star rating methods that is comprised of energy- and environmental-based metrics, potential vehicle classification systems, vehicle technology factors, and vehicle selection criteria.

The study tests several concepts and Energy Star rating methods using model-year 2000 vehicle data - a spreadsheet model has been developed to facilitate these analyses. This study tests two primary types of rating systems: (1) an outcome-based system that rates vehicles based on fuel economy, GHG emissions, and oil use and (2) a technology-based system that rates vehicles based on the energy-saving technologies they use. Rating methods were evaluated based on their ability to select vehicles with high fuel economy, low GHG emissions, and low oil use while preserving a full range of service (size and acceleration) and body style choice.

This study concludes that an Energy Star rating for passenger cars and light trucks is feasible and that several methods could be used to achieve reasonable tradeoffs between low energy use and emissions and diversity in size, performance, and body type. It also shows that methods that consider only fuel economy, GHG emissions, or oil use will not select a diverse mix of vehicles. Finally, analyses suggest that methods that encourage the use of technology only, may result in increases in acceleration power and weight rather than reductions in oil use and GHG emissions and improvements in fuel economy. 



\section{INTRODUCTION}

The Energy Star program is an eco-labeling program jointly administered by the U.S. Environmental Protection Agency (EPA) and the U.S. Department of Energy (DOE). The objective of this program is to reduce energy use and carbon dioxide emissions by encouraging the use and manufacture of energy-saving consumer products. To date, Energy Star ratings are available for over 30 product categories, and over 630 million of these products have been sold since the program began.

Cars and light trucks are a logical consideration for the Energy Star program, since these vehicles alone account for roughly one-sixth of the $\mathrm{CO}_{2}$ emissions in the United States and most of the petroleum consumed as fuel. Thus, vehicle fuel efficiency improvements can significantly reduce petroleum consumption and greenhouse gas (GHG) emissions.

Oak Ridge National Laboratory, under the sponsorship of the U.S. Department of Energy (DOE) Office of Energy Efficiency and Renewable Energy (EERE) Weatherization and Intergovernmental Program, has investigated the possible application of Energy Star ratings to passenger cars and light trucks. This study has produced an Energy Star framework for rating these vehicles, along with a spreadsheet model for analyzing potential Energy Star metrics and methods.

This is an exploratory study. Any decision to implement an Energy Star system for highway vehicles would involve broader consultation and consensus-building as well as additional analysis.

\subsection{BACKGROUND}

Energy Star is a voluntary labeling program established to reduce carbon dioxide $\left(\mathrm{CO}_{2}\right)$ emissions by promoting energy-saving products. The program promotes energy conservation, highlighting the cost savings that can be realized by using energy-efficient products. It also helps consumers identify these products by allowing manufacturers to affix an Energy Star Label to those that meet established efficiency criteria. Increased consumer demand encourages manufacturers to produce energy-efficient products, and the Energy Star label allows them to differentiate their products in the marketplace.

Although the program's initial objective was reducing $\mathrm{CO}_{2}$ emissions, it has grown to incorporate the parallel goal of reducing energy use, and there is new interest in reducing energy derived from petroleum. In promoting Energy Star, EPA Administrator Christie Whitman states, "Energy efficiency...can help preserve our natural resources, reduce foreign oil imports, save us billions of dollars, clean our air, and protect our planet for future generations."1

Energy Star was introduced by the US Environmental Protection Agency in 1992. The first Energy Starlabeled products were products such as personal computers and monitors, printers, and fax machines. EPA later partnered with the US Department of Energy in 1996 to promote the Energy Star label, with each agency taking responsibility for particular product categories. Energy Star has expanded to cover new homes, most of the buildings sector, residential heating and cooling equipment, major appliances, office equipment, lighting, consumer electronics, and other product areas. Energy Star ratings are available for over 30 product categories, and over 630 million of these products have been sold since the program began.

Energy Star maintains the attractiveness of labeled products by requiring them to meet quality, performance, and longevity criteria, and by making the Energy Star label available to products with features that consumers

\footnotetext{
${ }^{1}$ Environmental Protection Agency. 2001. Join Us In Making A Change. (Energy Star Fact Sheet) http://www.epa.gov/nrgystar/newsroom/pdf/estarchange9.pdf.
} 
want. One of the principal tenets of the Energy Star philosophy is that Energy Star products should maintain customer satisfaction by increasing energy efficiency without sacrificing product performance and features.

\subsection{PRODUCT CRITERIA}

Products qualify for the Energy Star label by meeting criteria established by EPA and DOE. The types of criteria used for obtaining the Energy Star label vary by product. Most use one or a combination of the following three criteria types.

Technology-based: The criteria for some products are technology-based: products qualify by incorporating the specified energy-saving technology or features. For example, this kind of criterion is used for programmable thermostats. A programmable thermostat is a temperature-sensitive device that lets the consumer choose the temperature to be maintained in one or several rooms of a home during different times of the day. These products are not required to meet any specific energy-efficiency requirements. They need only incorporate the features specified in the eligibility criteria: They must be capable of maintaining 2 separate programs to address the different comfort needs of weekdays and weekends, have up to 4 temperature settings for each program, and be capable of maintaining room temperature swings within $\pm 2^{\circ} \mathrm{F}$.

Absolute Efficiency: Some Energy Star criteria are defined in terms of absolute energy consumption rates during a mode of operation. For the purposes of our discussion, absolute means that the consumption rate is a fixed value independent of the quantity or quality of benefit provided by the product. For example, Energy Star-labeled home audio products must consume 2 watts or less when switched off, and TV and DVD products must consume 3 watts or less when switched off. The rate is the same regardless of the size of the television, output capacity of the amplifier, or the features provided by the DVD player. It should be noted that this type of criteria typically applies to a product's operation in "standby" or "sleep" mode, when only a few low-power functions remain active.

Efficiency Relative to Benefit: For some products, the key criterion may be established as a ratio of benefit or service (e.g., Btus of heating or cooling supplied) per amount of energy used. For example, residential central air conditioners are rated based on the Seasonal Energy Efficiency Ratio (SEER) and the Energy Efficiency Ratio (EER). SEER represents the total cooling (in Btus) during the normal cooling season as compared to the total electric energy input consumed (in watt-hours). Energy Efficiency Ratio (EER) is a measure of the instantaneous energy efficiency of cooling equipment measured as the steady-state rate of heat energy removal (e.g., cooling capacity) in Btus divided by the steady-state rate of energy use in watts. Within this category of criteria, the required efficiency rates are typically established based on (1) a product's rank in its category/class or (2) its improvement over existing standards:

- Rank in Product Category/Class. Some criteria are based on a product's energy efficiency rank within its respective market. For example, commercial buildings that are among the top 25 percent nationwide in terms of energy performance (earning a benchmarking score of 75 or greater) and maintain an indoor environment that conforms to industry standards can qualify for the Energy Star label. A building's energy efficiency is determined by the amount of energy used and other variables that affect energy use, such as building size, number of workers, number of computers, geographical location, weather, and the principal building activity. This data is then entered into a linear regression equation developed by DOE using historical energy consumption data from the 1992 and 1995 Commercial Buildings Expenditures and Consumption Survey (CBECS) conducted by the Energy Information Administration (EIA). If a building is more efficient than 75 percent of the buildings with similar characteristics, it is awarded an Energy Star.

- Improvement Over Existing Standards. Some Energy Star criteria, especially those for appliances, are set as a percent improvement over existing federal standards. For example, Energy Star refrigerators 
must achieve an energy use level 10 percent less than the National Appliance Energy Conservation Act (NAECA) standard. As the standards become more stringent, the Energy Star criteria are typically tightened also.

Combined Criteria: Many of the Energy Star criteria use a combination of the above rating methods. For example, computers must meet two criteria: one that is feature- or technology-based and another that is relative to service. First, an Energy Star computer must be capable of entering a "sleep" or "standby" mode after a given period of inactivity and "waking up" when the mouse is moved or a key is touched. Second, while in sleep mode, they must "power down" to 15 percent of their maximum power use. In this case, maximum power use is used as a surrogate for service since a computer's power supply gives a good indication of the features and computing power of the device.

\subsection{PRODUCT CLASSES}

Some Energy Star criteria further divide product categories into product classes. Product classes can be based on size, capacity, available features, or other attributes. For example, the refrigerator product category is divided into five different classes based on configuration and features (Table 1). Energy efficiency for all classes is measured in $\mathrm{kW}-\mathrm{hr} /$ year; the energy efficiency requirements are all a function of a refrigerator's volume; and all must achieve an energy use level 10 percent less than the National Appliance Energy Conservation Act (NAECA) standard. However, the energy efficiency algorithm for each class is different. By basing criteria on product class, Energy Star allows consumers more choice and allows a product to compete with other products having similar features.

Table 1. Energy Star Criteria for Refrigerators by Class

NAECA Maximum Energy Use

Product Class $(\mathrm{kWh} /$ year $)$

Top-mount freezer without through-the-door ice $9.8 \times$ Adjusted Volume +276

Side-mount freezer without through-the-door ice $4.91 \times$ Adjusted Volume +507.5

Bottom-mount freezer without through-the-door ice $4.6 \times$ Adjusted Volume +459

Top-mount freezer with through-the-door ice $10.2 \times$ Adjusted Volume +356

Side-mount freezer with through-the-door ice $10.1 \times$ Adjusted Volume +406

Adjusted Volume $=$ Fresh Volume $+(1.63 \times$ Freezer Volume $)$.

\subsection{RAISING STANDARDS FOR EVOLVING TECHNOLOGY}

All Energy Star criteria are subject to revision. As energy-saving technologies advance, the requirements for obtaining Energy Star status are strengthened so that only the most efficient products qualify. Energy Star criteria are established such that no more than 25 percent of the products in any given product category qualify for the label.

\subsection{ADDITIONAL CRITERIA}

In addition to energy- and environment-related criteria, most products must meet additional criteria to be awarded the Energy Star label. These additional criteria often include product quality standards, such as 
warranties, and performance requirements. These requirements assure that the Energy Star label is associated with quality, dependable products and that consumers can buy these products with confidence. This is especially important when promoting new technologies. Compact fluorescent light (CFL) bulbs, for example, must meet a myriad of additional photometric, electrical, and lifetime performance requirements. This facilitates market penetration by ensuring that consumers have positive experiences with this new technology. In addition, positive experiences with one Energy Star product increase the probability of consumers trying other such products. 


\section{ENERGY STAR FOR HIGHWAY VEHICLES}

Cars and light trucks are a logical consideration for the Energy Star program. These vehicles alone account for roughly one-sixth of the $\mathrm{CO}_{2}$ emissions in the United States and most of the petroleum consumed as fuel. Thus, vehicle fuel efficiency improvements can significantly reduce petroleum consumption and greenhouse gas (GHG) emissions.

The objective of this study is to explore potential Energy Star rating systems for highway vehicles. Two primary types of systems are discussed: (1) an outcome-based system that rates vehicles based on fuel economy improvements, reductions in greenhouse gas emissions, and/or reductions in oil use and (2) a technology-based system that rates vehicles based on the kinds of energy-saving technologies or features employed. A useful rating system would allow consumers to choose vehicles that emit less carbon dioxide, use less energy, and/or use less petroleum with modest or no sacrifice in quality, performance, or features.

This is an exploratory study. Any decision to implement an Energy Star system for highway vehicles would involve broader consultation and consensus-building as well as additional analysis. Furthermore, non-energyrelated product quality and performance criteria, while important elements of a successful Energy Star rating system, are beyond the scope of this study. They should, however, be explored if an Energy Star labeling system were established for highway vehicles. Additional criteria could require that vehicles meet specified safety, crash test, warranty, and/or emissions standards, or any other criteria that would ensure the Energy Star label is only awarded to quality products. 



\section{POTENTIAL ENERGY STAR METRICS FOR VEHICLES}

\subsection{THE LIMITATIONS OF SIMPLE MPG}

DOE and EPA already provide information to the public for comparing the energy efficiency of passenger cars and trucks based on miles per gallon (MPG). It is a useful metric, and most consumers understand and use it. However, using MPG alone to rate the efficiency of vehicles has a few limitations, especially for use in an Energy Star labeling system.

The MPG metric reflects only the distance a vehicle will go on a gallon of fuel, without reference to other kinds of service provided by the vehicle. Characteristics such as passenger and cargo volume, horsepower, four-wheel drive capability, and payload and towing capabilities are also important to consumers and are part of the purchase decision. Since weight and horsepower are the principal determinants of MPG, vehicles with the highest MPG are typically smaller passenger cars with smaller engines. However, these vehicles may not be a practical alternative for some consumers. It's also possible that using this as an Energy Star rating system would encourage manufacturers to spend more resources on improving the energy efficiency of smaller cars while concentrating less on that of larger vehicles, such as trucks and SUVs.

In addition, the MPG metric alone also doesn't account for the source of the energy used to power a vehicle. With renewed interest in decreasing U.S. oil dependence, it would be useful to develop a metric that would capture the benefit of using non-petroleum or low-carbon fuels as well. If an appropriate metric were identified, an Energy Star rating system could be designed to give extra weight to cars and trucks using nonpetroleum or low-carbon fuels.

\subsection{ESTABLISHING ENERGY STAR METRICS FOR CARS AND LIGHT TRUCKS}

More useful metrics would capture vehicle GHG emissions, energy use, and petroleum use and consider, at least to some degree, the service provided by the vehicle. This would allow consumers to choose among similar vehicles without sacrificing quality, performance, or features. Unfortunately, many vehicle characteristics such as size, weight, and horsepower negatively affect fuel economy. The challenge is to find the proper trade-off between improved energy and environmental performance and sacrifices in consumer choice.

Given Energy Star's objectives of reducing GHGs, energy use, and petroleum consumption, three fundamental metrics, or "measures of merit," can be used, either as a basis for Energy Star ratings for cars and light trucks or as components of a more comprehensive metric for Energy Star ratings:

- Annual Tons of GHG: Since one of the primary objectives of Energy Star is to reduce emissions of $\mathrm{CO}_{2}$ (the most significant manmade greenhouse gas), it would be useful to include a measure that, in part, rates vehicles based on the amount of GHGs they produce. Other GHGs emitted by vehicles include methane $\left(\mathrm{CH}_{4}\right)$ and nitrous oxide $\left(\mathrm{N}_{2} \mathrm{O}\right)$. When calculating $\mathrm{GHGs}$, it is important to consider the full fuel cycle emissions: both emissions produced by the vehicle and those emitted in producing the fuel

- Miles per Gallon: Energy efficiency measured in distance traveled per fuel consumed - typically, in terms of miles per gallon-is still a useful metric when coupled with other metrics or used to compare vehicles with similar features (e.g., comparing vehicles within a class). 
- Annual Barrels of Petroleum Consumed: Continued reliance on petroleum from foreign countries negatively affects our national energy security. Therefore, it would be appropriate to formulate a rating system that rewards vehicles that use less petroleum.

These measures of merit can be used alone, in combination with one another, and/or in combination with service measures described in the following section.

Other metrics could be used, such as criteria pollutant emissions or vehicle life-cycle impacts. However, criteria pollutant emissions are already strictly regulated and are not directly correlated with energy use. On the other hand, while embodied energy and vehicle life-cycle impacts would be useful, the data are not generally available. ${ }^{2}$

\subsection{COMPARING SIMILAR VEHICLES}

The common limitation of the above metrics is that they assume that the only service offered by a vehicle is moving itself from one place to another, each metric placing a premium on moving the vehicle the greatest distance for the least amount of impact, be it energy use, oil use, or GHG emissions. However, vehicles differ in the number of passengers and amount of cargo they can haul, the comfort they provide, the ability to travel on different types of terrain or in inclement weather, comfort (e.g., roominess, climate control, smoothness of ride, features), power, handling, and other attributes. These attributes are important to consumers and impact their purchasing decisions, usually much more than fuel economy or environmental impact. Therefore, it would be useful to construct an Energy Star framework that allows consumers to choose the most energyefficient vehicle that meets their needs. Vehicles are complex consumer products, and it would be infeasible to consider every conceivable difference among vehicles - no current Energy Star system accounts for every product difference. However, a few important vehicle attributes should be taken into consideration, especially those that have an impact on fuel efficiency.

Vehicle characteristics can be factored into an Energy Star rating method in two ways. First, vehicles can be grouped into product classes so that measures of merit for similar vehicles are compared. This method is used for several Energy Star product categories, such as commercial buildings, refrigerators, and room-unit air conditioners. Second, metrics that reflect the service offered by these products (e.g., size and/or power) can be combined with the measures of merit discussed above. Energy Star criteria for several products, such as central air conditioners, computers, and dehumidifiers, use a similar concept.

\subsubsection{Vehicle Classes}

One way to compare the energy-saving potential of similar vehicles is to divide them into classes based on one or more characteristics. Four vehicle groupings are used in this study:

- All: In this grouping, vehicles are compared to the entire fleet regardless of body style, size, features, or other attributes. This grouping method is used primarily to test for the optimal average score for each measure of merit or used with service-based metrics that account for vehicle differences.

- Car vs. Truck: This simple classification system recognizes that cars and trucks offer different services. While vans and sport utility vehicles (SUVs) are classified as trucks, they could logically be classified as either.

\footnotetext{
${ }^{2}$ DeCicco, J. and J. Kliesch. 2001. ACEEE's Green Book: The Environmental Guide to Cars and Trucks—Model Year 2001. American Council for an Energy-Efficient Economy, Washington. DC.
} 
- Type- and size-based classes: A common method of classifying vehicles is to group them based on type (function) and size (in terms of volume or gross vehicle weight rating). For this study, we used a hybrid system based on the Environmental Protection Agency (EPA) and California Energy Commission (CEC) classification systems. The EPA system was used for cars, since it contains a more detailed breakdown of car body types - it has three classes of station wagon, while CEC doesn't group station wagons separate from other cars. The CEC system was used for trucks, since it provides a more detailed breakdown for those vehicles, particularly in terms of size. The type-size class system used for this study is presented in the table below.

Table 2. Type-Size Classes

\begin{tabular}{|c|c|c|c|c|}
\hline \multicolumn{2}{|c|}{ Cars } & \multicolumn{3}{|c|}{ Trucks } \\
\hline Class & $\begin{array}{l}\text { Passenger \& } \\
\text { Cargo Volume } \\
\text { (cu feet) }\end{array}$ & Class & $\begin{array}{l}\text { Passenger \& } \\
\text { Cargo Volume } \\
\text { (cu feet) }\end{array}$ & $\begin{array}{c}\text { Gross Vehicle } \\
\text { Weight Rating } \\
\text { (lbs) }\end{array}$ \\
\hline 2-seater & Any & Compact Pickup & & $<4,500$ \\
\hline Mini & $<85$ & Standard Pickup & & $4,500-8,500$ \\
\hline Sub Compact & $85-99$ & Compact Van & $<=170$ & \\
\hline Compact & $100-109$ & Standard Van & $>170$ & \\
\hline Mid Size & $110-119$ & Mini Utility & $<120$ & \\
\hline Large & $>119$ & Compact Utility & $120-170$ & \\
\hline Small Wagon & $<130$ & Standard Utility & $>170$ & \\
\hline Mid Size Wagon & $130-159$ & & & \\
\hline Large Wagon & $>159$ & & & \\
\hline
\end{tabular}

- $\quad$ Market Classes: This system classifies vehicles based on body style, size, price, and features (e.g., 4wheel drive capability and convertible top). The market classes are somewhat less systematic, allowing vehicles to belong to more than one group. The advantage of this classification is that it reflects the service provided by different body styles, features, and luxury levels (although it uses price as a surrogate for luxury). The market-based vehicle classes are provided below.

Table 3. Market-based Vehicle Classes

\begin{tabular}{lll}
\hline Small Cars & Convertibles & Pickups \\
Coupes (2-doors) & Sporty Cars & Minivans \\
Family Sedans & Upscale Sedans & Sport Utility Vehicles \\
Large Sedans & Luxury Sedans & Four-wheel \& All-wheel Drive \\
Wagons & & \\
\hline
\end{tabular}

\subsubsection{Service-Based Metrics}

An alternative to grouping vehicles into classes is to choose variables that measure the service offered by different vehicle groups. Motor vehicles possess numerous attributes of importance to consumers, some which are easily measured and some which are not. Furthermore, since vehicles are complex consumer goods, no 
single measure can be expected to fully reflect all aspects of a vehicle's value to consumers except, possibly, price. There is no precedent, however, for basing Energy Star ratings on the price of energy-using equipment. Still, several metrics that represent various aspects of a vehicle's service can be easily quantified, such as body style, interior volume, payload, power, four-wheel drive capability, and seating capacity. This study uses just a few service indicators that can be calculated quickly using available data. It is likely that, once an Energy Star system was established, manufacturers would be willing to supply any additional data needed.

- Size: While the sum of passenger and cargo volume would be a desirable measure of a vehicle's capacity, no volume data is readily available for SUVs and vans. However, an alternative measure for size can be readily computed from available data. A vehicle's footprint is defined as the area calculated multiplying a vehicle's width by its wheelbase. Footprint is closely related to several vehicle attributes such as external size, weight, and passenger volume. Preliminary analysis by ORNL has shown that footprint is a good predictor of volume.

- Performance: This metric is based on a vehicle's acceleration time from 0 to 60 and is calculated based on a vehicle's horsepower-to-weight ratio.

In this study, the footprint metric is combined with the measures of merit to form the following metrics:

- Footprint-MPG: Defined as the product of MPG and footprint (with units of square-feet-miles per gallon), this measure incorporates vehicle size and fuel efficiency.

- Oil Use per Footprint: Defined as oil use divided by footprint (with units of annual barrels of oil used per square foot), this measure incorporates oil use and vehicle size.

- Oil Use \& GHG per Footprint: Defined as oil use and GHG emissions per footprint, this metric represents the ratio of GHG emissions and oil use to vehicle size. Since GHG is closely related to energy use, this metric secondarily considers energy use as well. 


\section{OUTCOME-BASED ENERGY STAR CRITERIA EXPLORED}

\subsection{APPROACH}

The Energy Star framework described above has been implemented in a spreadsheet model, making it easy to test and modify various Energy Star rating methods. This model uses EPA vehicle fuel economy data for model year 2000, which has been enhanced by EEA and ORNL.

Several outcome-based Energy Star rating methods were explored in this study. For all methods, vehicles scoring in the top 20 percent for the given metric were selected as Energy Star vehicles. Energy Star guidelines state that no more than 25 percent of a given product type should receive an Energy Star rating. Therefore, a slightly lower percentage was chosen for this study. The software tool developed for this study allows the analyst to specify the percentile of vehicles to be selected, making it easy to generate a new set of qualifying vehicles.

The first four methods use metrics that represented energy and environmental attributes only. These metrics include individual vehicle fuel economy (MPG), annual GHG emissions (in tons), annual oil use (in barrels), and a weighted average score based on GHG emissions and oil use. No attempt was made to consider other forms of service offered by the vehicles or consumer choice, either by using service metrics or by grouping vehicles into classes. These methods provide an estimate of the best average MPG, GHG, and oil use score realized from using each metric.

For the next group of methods $(5,12-14)$, measures of merit were combined with size and performance metrics that represent, to some degree, service and consumer choice. By considering the services provided by vehicles, these methods should make Energy Star choices available across a wider range of vehicle types than methods using measures of merit only. The following metrics are examined in this study:

- Footprint-MPG, which considers fuel economy and vehicle size

- $\quad$ Oil Use per Footprint, which considers oil use and vehicle size

- Oil Use and GHG Emissions per Footprint, which considers oil use, GHG emissions, and vehicle size

- Oil Use and GHG Emissions per Footprint (normalized), which considers oil use, GHG emissions, and vehicle size, but attempts to normalize the oil use and GHG emissions so that they are given equal weight

In addition to these metrics, a regression analysis was performed to determine the relationship between various vehicle characteristics and MPG. The resulting equations were used to predict each vehicle's MPG based on weight, horsepower, and body type (Methods 6 and 7). Vehicles were rated based on how their actual MPG compared to the MPG predicted by the model. These methods are discussed in more detail in the "Results" section that follows. Like the previously described methods, vehicle models were not divided into classes.

Finally, vehicle models were grouped into classes using three different classification systems: body type (i.e., car vs. truck), type-size class, and market class (Methods 8-11). The vehicle models scoring in the top 20 percent in each vehicle class for the specified metric were selected for Energy Star. This allowed each vehicle to compete against other vehicles with similar features (in varying degrees) and ensures that Energy Star choices are available across a wide range of vehicle types. The primary metric examined for each class system was MPG. The GHG metric was also used with the type-size classification system. 
The matrix in the table below shows the metrics and vehicle classification systems used for each method. The results for each method are presented in the following section.

Table 4. Matrix of Metrics and Vehicle Classes

\begin{tabular}{lcccc}
\hline & \multicolumn{3}{c}{ Vehicle Classification Systems } \\
\cline { 2 - 5 } Metric & $\begin{array}{c}\text { All } \\
\text { Vehicles }\end{array}$ & $\begin{array}{c}\text { Car vs. } \\
\text { Truck }\end{array}$ & $\begin{array}{c}\text { Type-Size } \\
\text { Class }\end{array}$ & $\begin{array}{c}\text { Market } \\
\text { Class }\end{array}$ \\
\hline MPG & 1 & 8 & 9 & 11 \\
Annual GHG emissions & 2 & & 10 & \\
Annual petroleum use & 3 & \\
Weighted sum of GHG \& petroleum use & 4 & \\
Footprint-Miles/Gallon (ft' ${ }^{2}$-mi/gal) & 5 & \\
Ratio of actual to predicted MPG from regression model: Eq. & 6 & \\
1 (curb weight \& horsepower) & & \\
Ratio of actual to predicted MPG from regression model: Eq. & 7 & \\
2 (curb weight, horsepower \& car/truck) & & \\
Oil Use/Footprint & 12 & \\
(GHG \& Oil Use)/Footprint & $13 \& 14$ & \\
\hline
\end{tabular}

\subsubsection{Method 1: Best fuel economy (MPG)}

Method 1 ranks vehicles based on fuel economy only; vehicle models with MPG in the top 20 percent are selected for Energy Star. Vehicles are not divided into vehicle classes, and the chosen metric (MPG) alone does not consider vehicle service or choice. Fuel economy estimates for gasoline- and diesel-powered vehicles are measured in miles per gallon, while estimates for alternative fuel, flexible fuel, and dual fuel vehicles are measured in miles per gasoline gallon equivalent. Flexible and dual fuel vehicles were assumed to operate 50 percent of the time on each fuel type. All vehicles were assumed to travel 15 thousand miles annually.

\subsubsection{Method 2: Lowest Annual GHG Emissions}

Method 2 ranks vehicles based on annual emissions of GHGs only; vehicle models with GHG emissions in the lowest 20 percent are selected for Energy Star. Vehicles are not divided into vehicle classes, and the metric does not consider vehicle service or choice. GHG estimates were estimated using fuel specifications and emissions estimates from the Greenhouse Gases, Regulated Emissions, and Energy Use in Transportation (GREET) Model (vs. 1.5) developed by Argonne National Laboratory. ${ }^{3}$ Flexible and dual fuel vehicles were assumed to operate 50 percent of the time on each fuel type. All vehicles were assumed to travel 15 thousand miles annually.

\footnotetext{
${ }^{3}$ Argonne National Laboratory. 2002. The Greenhouse Gases, Regulated Emissions, and Energy Use in Transportation (GREET) Model (http://greet.anl.gov/default.htm).
} 


\subsubsection{Method 3: Lowest Annual Oil Use}

Method 3 selects the vehicle models that consume the least petroleum. Vehicles are not divided into vehicle classes, and the chosen metric alone does not consider vehicle service or choice. Annual oil use is estimated based on assumptions allowed to vehicle manufacturers in calculating CAFE as provided by Section 6 of the Alternative Motor Fuels Act and described in the U.S. Code (49 USC 32905). ${ }^{4,5}$ Full-cycle oil use rates for electric vehicles are estimated based on DOE's regulations for calculating petroleum-equivalent fuel economy of electric vehicles. ${ }^{6}$ Flexible and dual fuel vehicles were assumed to operate 50 percent of the time on each fuel type.

The assumptions regarding the petroleum content of alternative fuels, as put forth by this legislation, provide for favorable CAFE treatment of alternative fuel vehicles. Other assumptions could be used in the model to estimate the petroleum use of these vehicles based on empirical evidence.

\subsubsection{Method 4: Lowest Weighted Average of GHG and Oil Use Scores}

Method 4 rates vehicles based on the weighted average of their GHG and oil use scores as estimated in the previous two methods. In this method, GHG and oil use were given equal weight in selecting vehicles.

\subsubsection{Method 5: Highest Footprint-MPG}

Unlike Method 1, which considers only MPG, Method 5 also considers vehicle size by incorporating the vehicle footprint metric. Footprint-MPG is calculated as the footprint multiplied by the MPG. The resulting unit is square-feet-miles per gallon (sq. ft. mi./gal).

\subsubsection{Methods 6 \& 7: Best Ratio of Actual to Predicted MPG Based on Regression Analysis}

Method 6 selects Energy Star vehicles based on vehicle weight, horsepower, and MPG. Method 7 uses these vehicle characteristics along with basic vehicle body style (i.e., car vs. truck). For these two methods, data for model year 2000 vehicles was used to perform a linear regression analysis to determine the relationship between various vehicle characteristics and MPG. The resulting equation was used to predict the fuel efficiency of each vehicle. The equations used to predict MPG are given below. The predicted MPG was then compared to the unadjusted MPG for each vehicle. Vehicles with high actual-to-predicted MPG ratios were considered more efficient.

$$
\begin{aligned}
& \text { Predicted MPG for Method } 6=e^{\alpha} \times c u r b w t^{\beta} \times h p^{\delta} \\
& \text { Predicted MPG for Method } 7=e^{\alpha} \times c u r b w t^{\beta} \times h p^{\delta} \times e^{b o d y \times \varepsilon} \\
& \text { where }
\end{aligned}
$$

$$
\begin{aligned}
& c u r b w t=\text { the vehicle's curb weight } \\
& h p=\text { the vehicle's horsepower } \\
& b o d y=\text { vehicle body type ( } 0 \text { if car; } 1 \text { if truck) }
\end{aligned}
$$

\footnotetext{
${ }^{4}$ Environmental Protection Agency. 1994. Fuel Economy Test Procedures Alternative-Fueled Automobile CAFE Incentives and Fuel Economy Labeling Requirements; Final Rule. Federal Register, Vol. 59, August 3.

${ }^{5} 49$ U.S.C. Sec. 32905 (http://frwebgate4.access.gpo.gov/cgi-

bin/waisgate.cgi?WAISdocID $=14776724133+0+0+0 \&$ WAISaction=retrieve)

${ }^{6}$ Department of Energy, Office of Energy Efficiency and Renewable Energy. 2000. 10 CFR 474: Electric and Hybrid Vehicle Research, Development, and Demonstration Program; Petroleum-Equivalent Fuel Economy Calculation; Final Rule. Federal Register, Vol. 65, No. 113, June 12, pp. 36986-36992.
} 


$$
\begin{aligned}
& \alpha=\text { intercept }, 8.192504367 \\
& \beta=\text { curb weight coefficient, }-0.409064723 \\
& \delta=\text { horsepower coefficient, }-0.30842115 \\
& \varepsilon=\text { body type coefficient, }-0.131565343
\end{aligned}
$$

\subsubsection{Method 8: Best Fuel Economy (MPG) in Each Body Type Class (Car vs. Truck)}

This method selects vehicles with MPG in the top 20 percent of their respective class. For this method, vehicles are classified as either cars or trucks. The truck class includes pickups, vans, and SUVs.

\subsubsection{Method 9: Best Fuel Economy (MPG) in Each Type-Size Class}

This method selects vehicles with MPG in the top 20 percent of their respective type-size class. (Type-size classes are shown in Table 2.)

\subsubsection{Method 10: Least GHG Emissions in Each Type-Size Class}

This method selects vehicles with annual GHG emissions in the lowest 20 percent of their respective type-size class. (Type-size classes are shown in Table 2.)

\subsubsection{Method 11: Best Fuel Economy (MPG) in Each Market Class}

This method selects vehicles with MPG in the top 20 percent of their respective market class. (Market-based vehicle classes are shown in Table 3.)

\subsubsection{Method 12: Lowest Ratio of Oil Use to Footprint}

This method selects vehicles based on the ratio of oil use to vehicle size. Oil use is estimated as described under Method 3, and footprint is used to represent vehicle size. The result is in units of annual barrels of oil per square foot (bbls/sq. ft.). This method selects vehicles whose oil/footprint scores rank in the bottom 20 percent of all vehicles.

\subsubsection{Methods 13 and 14: Best Ratio of GHG and Oil Use to Footprint}

These methods select vehicles based on the ratio of GHG emissions and oil use to vehicle size. GHG emissions and oil use are estimated as described in Methods 2 and 3. Vehicle size is represented by the footprint metric. The difference between the two methods is that Method 14 normalizes the GHG emissions and oil use scores.

Method 13 rates each vehicle based on the following equation.

Score $=\frac{G H G+\text { Oil }}{\text { Footprint }}$

Method 14 normalizes the GHG and oil use values by taking each measure of merit, subtracting the mean of that metric for all vehicles, and dividing the quotient by the standard deviation for each metric.

Score $=\frac{\frac{G H G-\overline{G H G}}{\sigma_{G H G}}+\frac{\text { Oil }-\overline{O i l}}{\sigma_{\text {Oil }}}}{\text { Footprint }}$ 


\subsection{RESULTS}

The vehicles meeting the Energy Star criteria for each of the above methods were determined, and summary statistics were then calculated on the vehicles chosen by each. Statistics were generated for the measures of merit, vehicle attributes, and number of alternative fuel vehicles selected. These statistics are summarized in the following sections. A summary of each method's strengths and limitations is also included. A complete listing of the vehicles selected by each method is presented in Appendix B.

It should be noted that the tables and figures in this section also contain results for the technology-based Energy Star method discussed later in this report.

\subsubsection{Measures of Merit}

The average value for each measure of merit (i.e., fuel economy, GHG emissions, and oil use) was calculated for the vehicles selected by each method, along with the percent difference compared to the average for the "base case" (i.e., all 2000 model year vehicle models). The methods were then ranked against one another for each measure of merit. The results are presented in Figure 1 and Table 5.

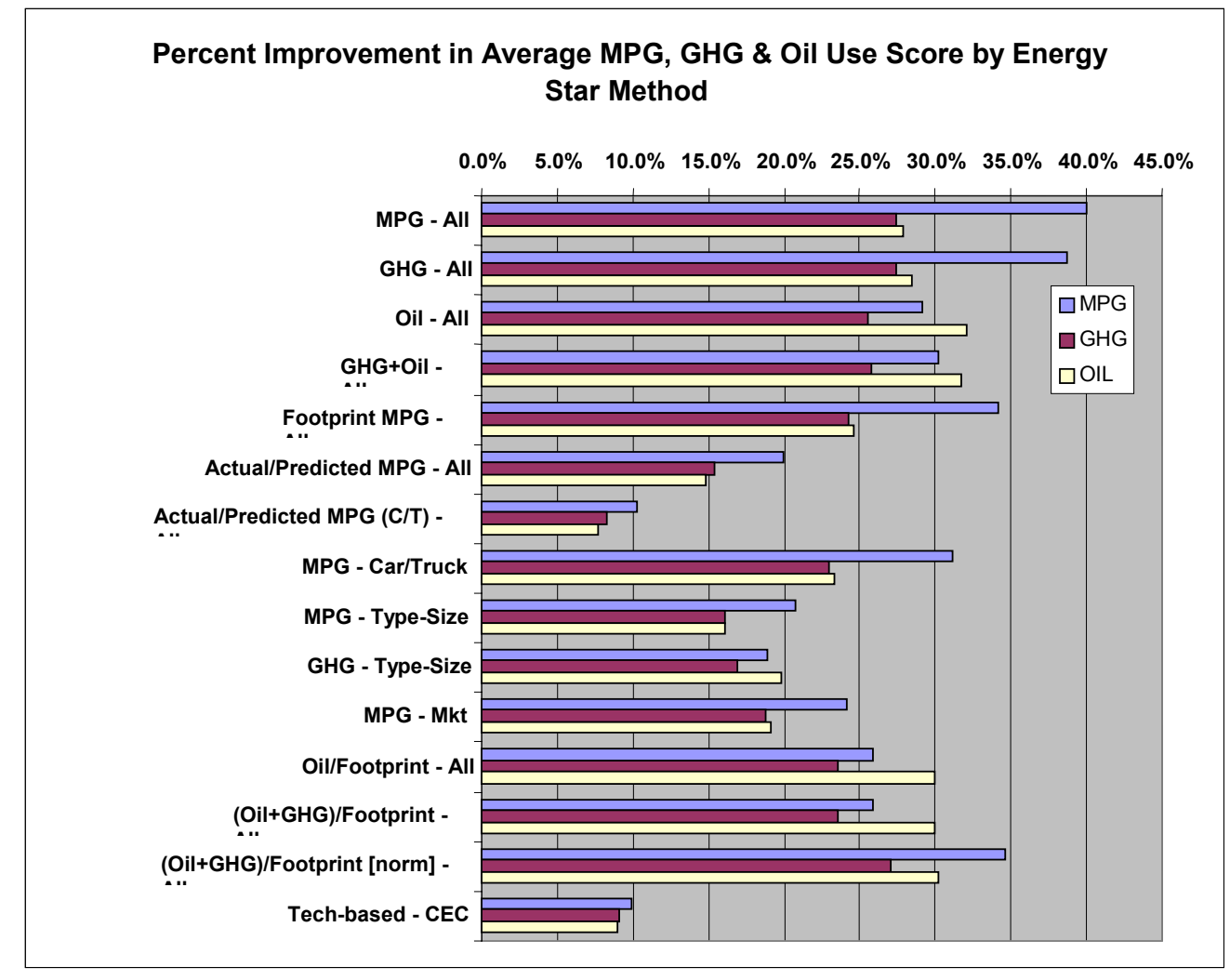

Figure 1. Percent Improvement in the Average MPG, GHG Emissions, and Oil Use Scores for Vehicles Selected by Each Energy Star Method 
Table 5. Average Measures of Merit for Vehicles Selected by Each Energy Star Rating Method

\begin{tabular}{ll|ccc|ccc|ccc}
\hline & & \multicolumn{3}{c}{$\begin{array}{c}\text { Fuel Economy } \\
\text { (miles/gallon) }\end{array}$} & \multicolumn{2}{c}{$\begin{array}{c}\text { GHG } \\
\text { (Ann. Tons GHG/Veh.) }\end{array}$} & \multicolumn{3}{c}{ Oil Use } \\
(Ann. BBLs Oil/Veh.)
\end{tabular}

\subsubsection{Vehicle Attributes}

Since one of the goals of the Energy Star program is to increase energy efficiency without sacrificing performance and features, statistics representing vehicle attributes that are important to consumers were also calculated for the selected vehicles. Although it is impossible to account for all attributes of interest to consumers, three basic characteristics were examined.

1. Vehicle size (represented by footprint). One of the trade-offs typically associated with improved fuel economy is decreased vehicle size. Smaller cars are usually more fuel-efficient but may be less desirable to some consumers. Therefore, the methods are scored based on the average size of the selected vehicles as represented by the footprint metric (Table 6 and Figure 2).

2. Acceleration. Another trade-off is decreased acceleration capability. Acceleration is typically a product of a vehicle's horsepower-to-weight ratio and is measured as the time it takes (in seconds) to go from a stopped position to a speed of 60 miles per hour $(\mathrm{mph})$. Methods were scored based on average acceleration time of the selected vehicles, with lower acceleration times receiving better scores (Table 6 and Figure 3). The acceleration time for each vehicle was estimated using the following equation. 
Acceleration $=e^{-0.00275322-0.77613136 \times \ln \left(\frac{H p}{W t}\right)}$

3. Body style. Body style is one of the most basic attributes that affect vehicle purchase decisions, and it is a good indicator of consumer choice and vehicle diversity. Ideally, a method should select a diverse mix of the many body styles popular in the vehicle market. For the sake of simplicity, this report presents statistics on body style in the most basic sense (i.e., car vs. truck), rather than a more detailed breakdown (e.g., small cars, station wagons, family sedans, sports cars, etc.).

Since the consumer demand for cars and trucks is roughly equal in the new-vehicle market, it would be desirable for consumers to have a choice of energy-efficient cars and trucks. Therefore, methods are judged, at least partially, on selecting a mix of cars and trucks that is representative of consumer demand and/or available manufacturer models (Table 6 and Figure 4). The metric used for this characteristic is percent of the selected vehicles that are trucks. In terms of the vehicle models available in model year 2000, 41.5 percent were trucks; in terms of sales, 44.9 percent were trucks. Methods that have a share of trucks close to 40-50 percent receive higher scores.

Table 6. Average Attribute Scores for Vehicles Selected by Each Energy Star Rating Method

\begin{tabular}{|c|c|c|c|c|c|c|c|c|c|c|}
\hline \multicolumn{2}{|c|}{ Method } & \multicolumn{3}{|c|}{$\begin{array}{c}\text { Footprint } \\
\text { (sq. ft. per vehicle) }\end{array}$} & \multicolumn{3}{|c|}{$\begin{array}{l}\text { Acceleration Time } \\
\text { (seconds) }\end{array}$} & \multicolumn{3}{|c|}{$\begin{array}{c}\% \text { of Vehicles that are } \\
\text { Trucks }\end{array}$} \\
\hline No. & Description & Avg. & $\%$ Chg & Rank & Avg. & \% Chg & Rank & Avg. & Chg & Rank \\
\hline 1 & MPG - All & 47.3 & $-11.2 \%$ & 15 & 10.9 & $-13.3 \%$ & 8 & $5.4 \%$ & $-36.1 \%$ & 14 \\
\hline 2 & GHG - All & 47.5 & $-10.9 \%$ & 13 & 11.0 & $-13.8 \%$ & 11 & $7.1 \%$ & $-34.4 \%$ & 13 \\
\hline 3 & Oil - All & 48.2 & $-9.4 \%$ & 11 & 11.2 & $-15.7 \%$ & 14 & $16.8 \%$ & $-24.7 \%$ & 9 \\
\hline 4 & GHG+Oil - All & 48.2 & $-9.4 \%$ & 10 & 11.1 & $-15.3 \%$ & 13 & $15.4 \%$ & $-26.1 \%$ & 10 \\
\hline 5 & Footprint MPG - All & 50.3 & $-5.6 \%$ & 8 & 10.6 & $-9.6 \%$ & 4 & $9.6 \%$ & $-31.9 \%$ & 12 \\
\hline 6 & $\begin{array}{l}\text { Actual / Predicted } \\
\text { MPG - All }\end{array}$ & 51.6 & $-3.1 \%$ & 2 & 8.7 & $10.0 \%$ & 1 & $0.6 \%$ & $-40.9 \%$ & 15 \\
\hline 7 & $\begin{array}{l}\text { Actual / Predicted } \\
\text { MPG }(\mathrm{C} / \mathrm{T}) \text { - All }\end{array}$ & 54.5 & $2.4 \%$ & 1 & 9.1 & $6.0 \%$ & 2 & $32.9 \%$ & $-8.6 \%$ & 6 \\
\hline 8 & MPG - Car/Truck & 47.6 & $-10.5 \%$ & 12 & 11.3 & $-16.6 \%$ & 15 & $41.5 \%$ & $0.0 \%$ & 3 \\
\hline 9 & MPG - Type-Size & 51.3 & $-3.6 \%$ & 5 & 10.8 & $-11.6 \%$ & 6 & $41.9 \%$ & $0.4 \%$ & 1 \\
\hline 10 & GHG - Type-Size & 51.4 & $-3.5 \%$ & 4 & 10.9 & $-12.4 \%$ & 7 & $41.6 \%$ & $0.1 \%$ & 2 \\
\hline 11 & MPG - Mkt & 48.9 & $-8.1 \%$ & 9 & 10.7 & $-10.4 \%$ & 5 & $36.6 \%$ & $-4.9 \%$ & 5 \\
\hline 12 & Oil/Footprint - All & 50.5 & $-5.2 \%$ & 6 & 11.0 & $-13.5 \%$ & 9 & $18.0 \%$ & $-23.5 \%$ & 7 \\
\hline 13 & $(\mathrm{Oil}+\mathrm{GHG}) /$ Footprint & 50.5 & $-5.2 \%$ & 6 & 11.0 & $-13.5 \%$ & 9 & $18.0 \%$ & $-23.5 \%$ & 7 \\
\hline 14 & $\begin{array}{l}(\mathrm{Oil}+\mathrm{GHG}) / \text { Footprint } \\
\text { [norm] }\end{array}$ & 47.4 & $-10.9 \%$ & 14 & 11.1 & $-14.6 \%$ & 12 & $12.0 \%$ & $-29.5 \%$ & 11 \\
\hline $\mathrm{T}$ & Tech-based-CEC & 51.5 & $-3.3 \%$ & 3 & 10.3 & $-6.6 \%$ & 3 & $40.8 \%$ & $-0.7 \%$ & 4 \\
\hline \multirow[b]{2}{*}{ Base } & \multirow[b]{2}{*}{ All vehicle models } & Avg. & \multicolumn{2}{|c|}{ Sales-wt. Avg. } & Avg. & \multicolumn{2}{|c|}{ Sales-wt. Avg. } & Avg. & \multicolumn{2}{|c|}{ Sales-wt. Avg. } \\
\hline & & 53.2 & 54.4 & & 9.7 & 10.2 & & 41.5 & 44.9 & \\
\hline
\end{tabular}




\section{Average Footprint for Each Energy Star Method}

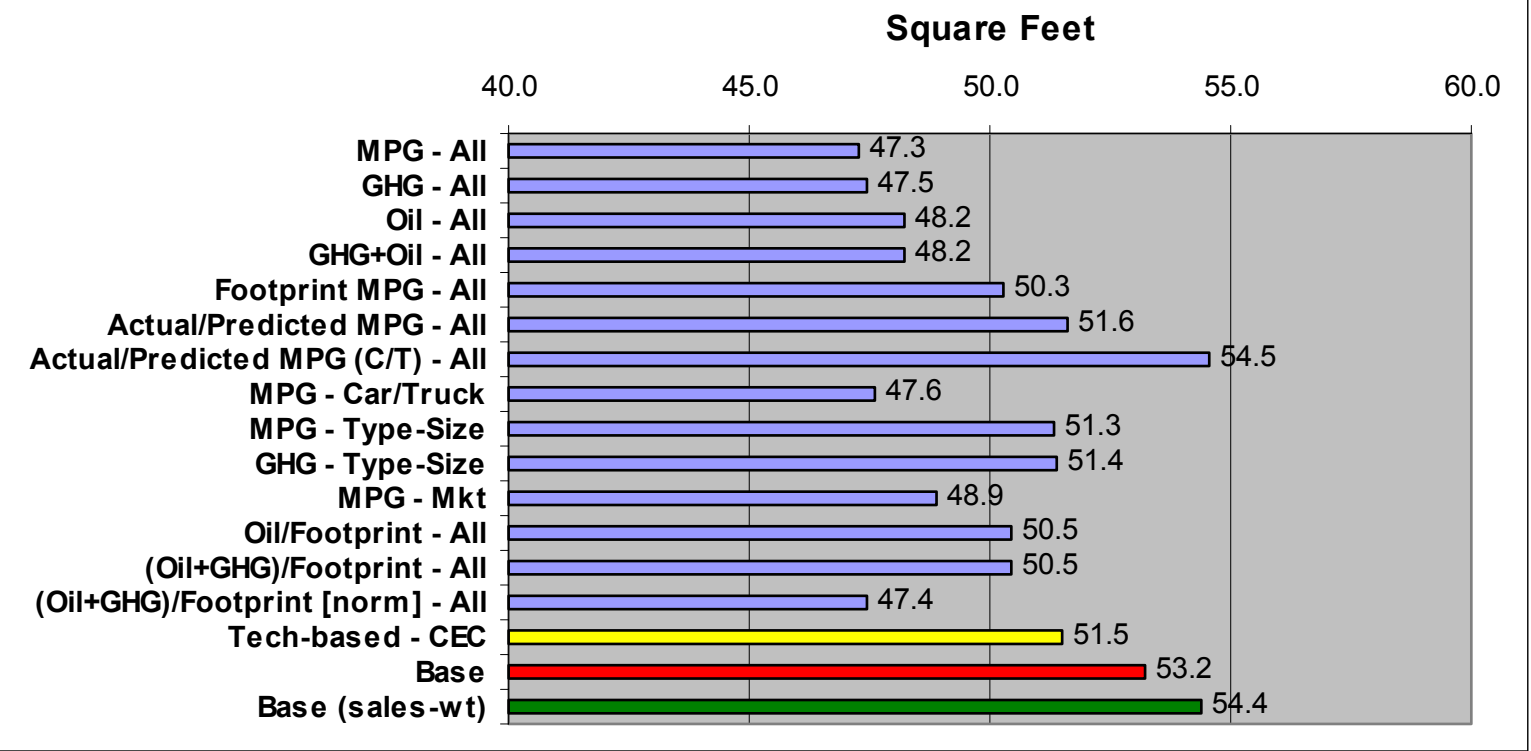

Figure 2. Average Footprint for Vehicles Selected by Each Energy Star Method

\section{Average 0-60 ( $\mathrm{mph}$ ) Acceleration Time for Each Energy Star Method}

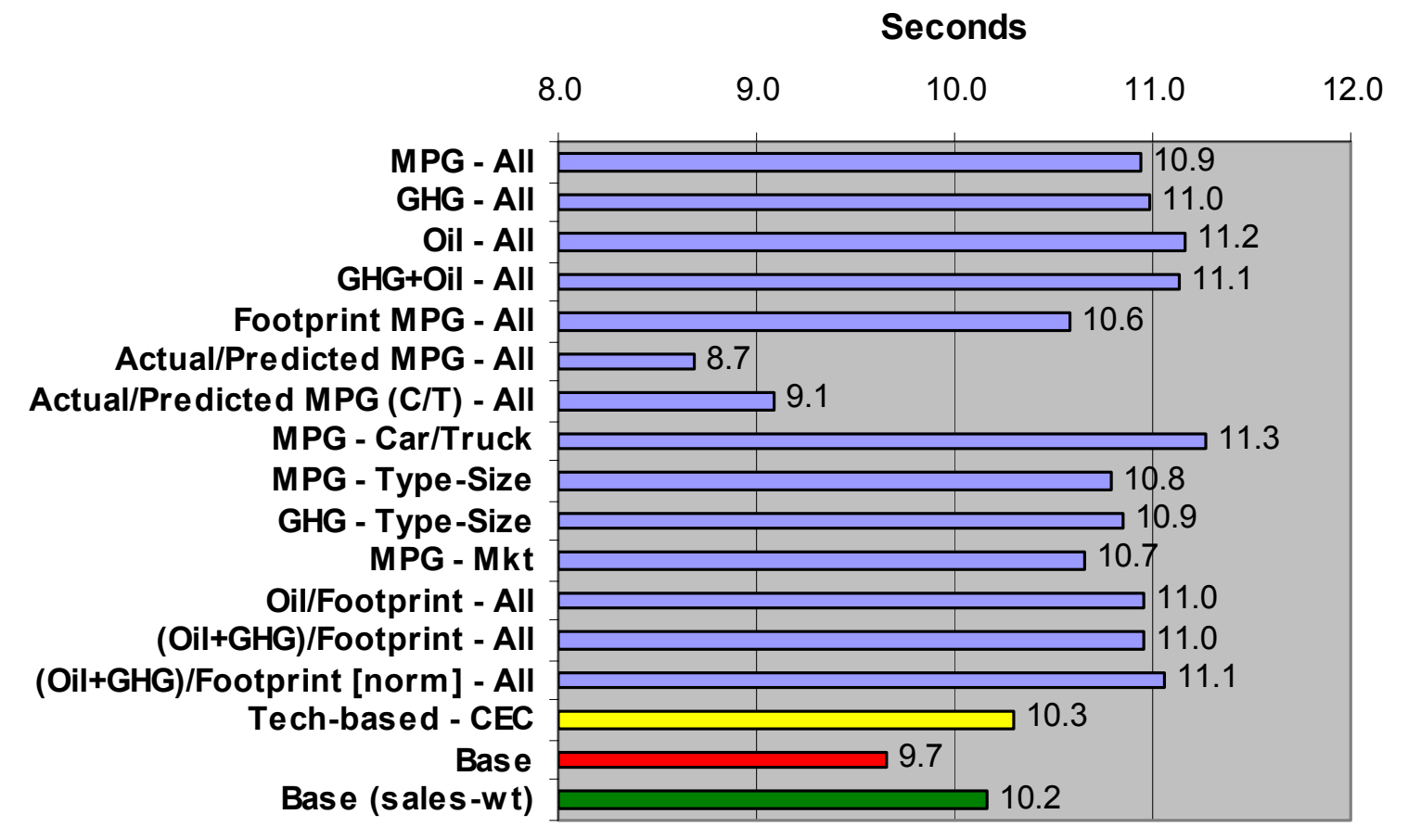

Figure 3. Average Acceleration Time for Vehicles Selected by Each Energy Star Method 


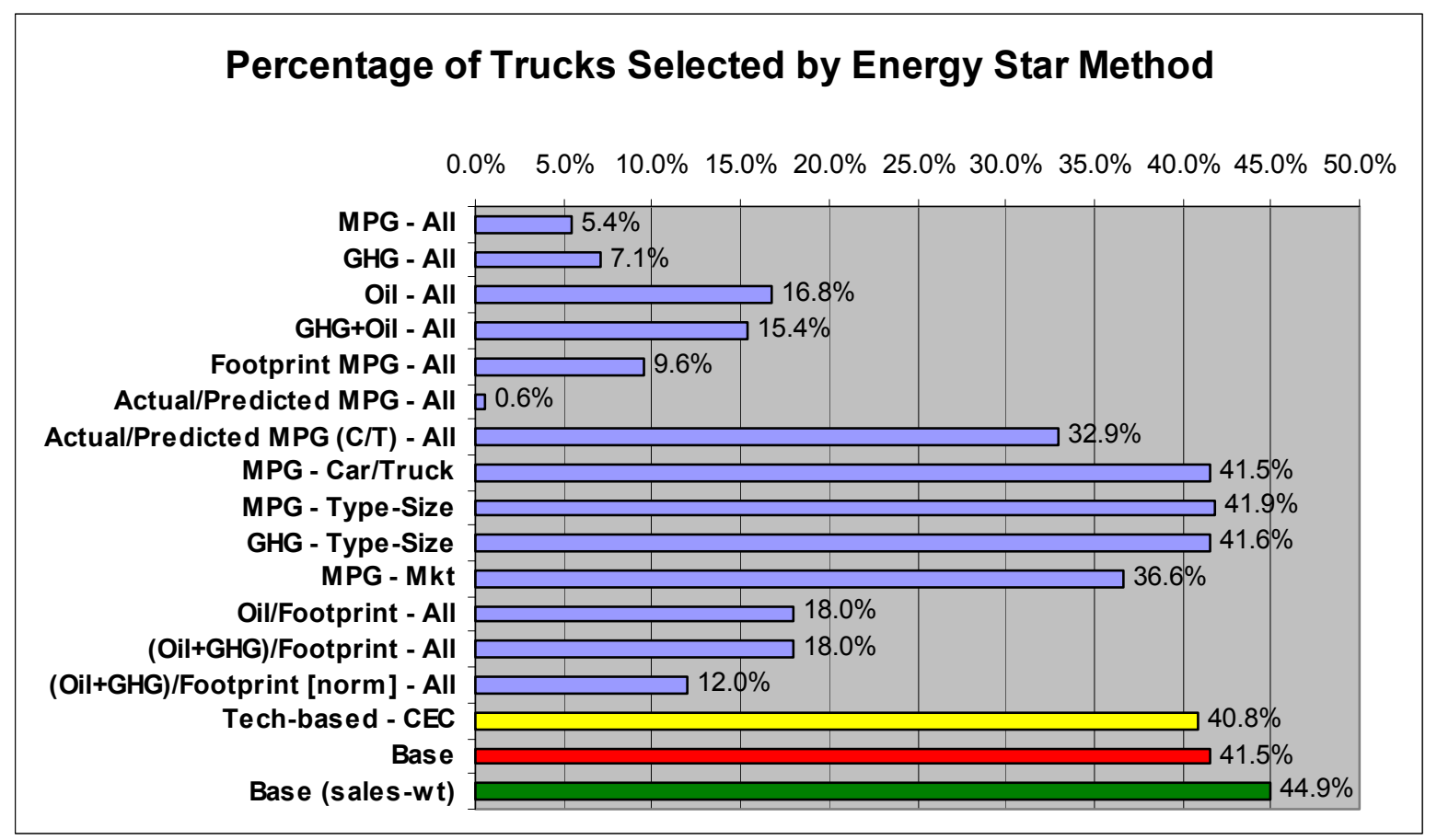

Figure 4. Percentage of Vehicles Selected by Each Energy Star Method that are Trucks

The attribute averages for each method were calculated and compared to the average for the base case. Two averages are calculated for the base case: the simple average and the sales-weighted average. The simple average is indicative of the characteristics of the vehicle models available, while the sales-weighted average is more indicative of consumer preference-vehicles with preferred attributes have higher sales. For example: On a model basis, the average acceleration time for all models produced by manufacturers is 9.7 seconds $(0$ to $60 \mathrm{mph}$ ). However, the sales-weighted average is 10.2, indicating that consumers may actually be satisfied with less acceleration power.

High average footprints and acceleration times are also good indicators of vehicle diversity. Most of the methods will automatically select the smallest vehicles and those with longer acceleration times (mostly due to low horsepower ratings). Higher averages for these scores indicate that larger vehicles with faster acceleration are also selected.

The sales-weighted average footprint for all model year 2000 vehicles was 54.4 sq. ft. Methods selecting a set of vehicles with non-sales weighted average of $52 \mathrm{sq}$. $\mathrm{ft}$. or greater were scored as "good." Examples of vehicles in the 52-56 sq. ft. range, regardless of whether they were selected for Energy Star, include the Honda Accord (52.2), Nissan Maxima (52.9), Ford Taurus (55.3), and Ford Ranger Pickup (53.8) and Jeep Grande Cherokee SUV (53.2).

Methods selecting a group of vehicles with an average footprint greater than or equal to $50 \mathrm{sq}$. $\mathrm{ft}$. and less than 52 sq. $\mathrm{ft}$. were considered to do a fair job of selecting vehicles close to the sales-weighted average. Examples of common vehicles in this size range include the Mercury Cougar (50.1), Ford Contour (50.9), Toyota Camry (51.2), Chevy S10 Pickup (51.1), and the Nissan Pathfinder SUV (51.5).

Methods selecting a set of vehicles with an average footprint of less than $50 \mathrm{sq}$. $\mathrm{ft}$. were considered to do a poor job of selecting vehicles close to the sales-weighted average. Common vehicles with a footprint just under 50 sq. ft. include the Honda Civic (48.1), Dodge Neon (49.1), Mitsubishi Gallant (49.8), Nissan Frontier Pickup (48.2), and Toyota 4Runner SUV (48.6). This is not so say that these vehicles are not of a desirable size, just that they represent the smaller end of their respective classes. 
Estimated acceleration time for all model year 2000 vehicles ranged from a minimum of 4.8 seconds (Dodge Viper, Ferrari Modena) to 17.8 seconds (Ford Ranger Electric Pickup). The average acceleration time for all models in MY2000 was 9.7 seconds. The sales-weighted average (10.2 seconds) suggests that consumers were content with somewhat less powerful vehicles. In examining each method, acceleration times of 10.5 or less were considered good, while average acceleration times greater than 10.5 seconds and less than or equal to 11.5 seconds were considered fair. Average acceleration times greater than 11.5 seconds were considered poor. However, no method selected vehicles with an average greater than 11.3 seconds.

Forty-one and a half (41.5) percent of the models available for model year 2000 were trucks, while 44.9 percent of vehicles sold were trucks. This is a good indicator of consumer preference in body style. Methods selecting 30-60 percent trucks were considered as providing a good mix of body styles (45 percent \pm 15 percent). Methods selecting between 15 and 30 percent trucks were considered offering a fair mix of body styles. Methods selecting less than 15 percent trucks were considered offering a poor mix of body styles.

\subsubsection{Alternative Fuel Vehicles}

The goal of reducing oil dependence makes alternative fuel vehicles of special significance. Therefore, the number of alternative fuel vehicles selected by each method was also calculated (Figure 5). Twenty-six (26) models that use alternative fuels were available in 2000.

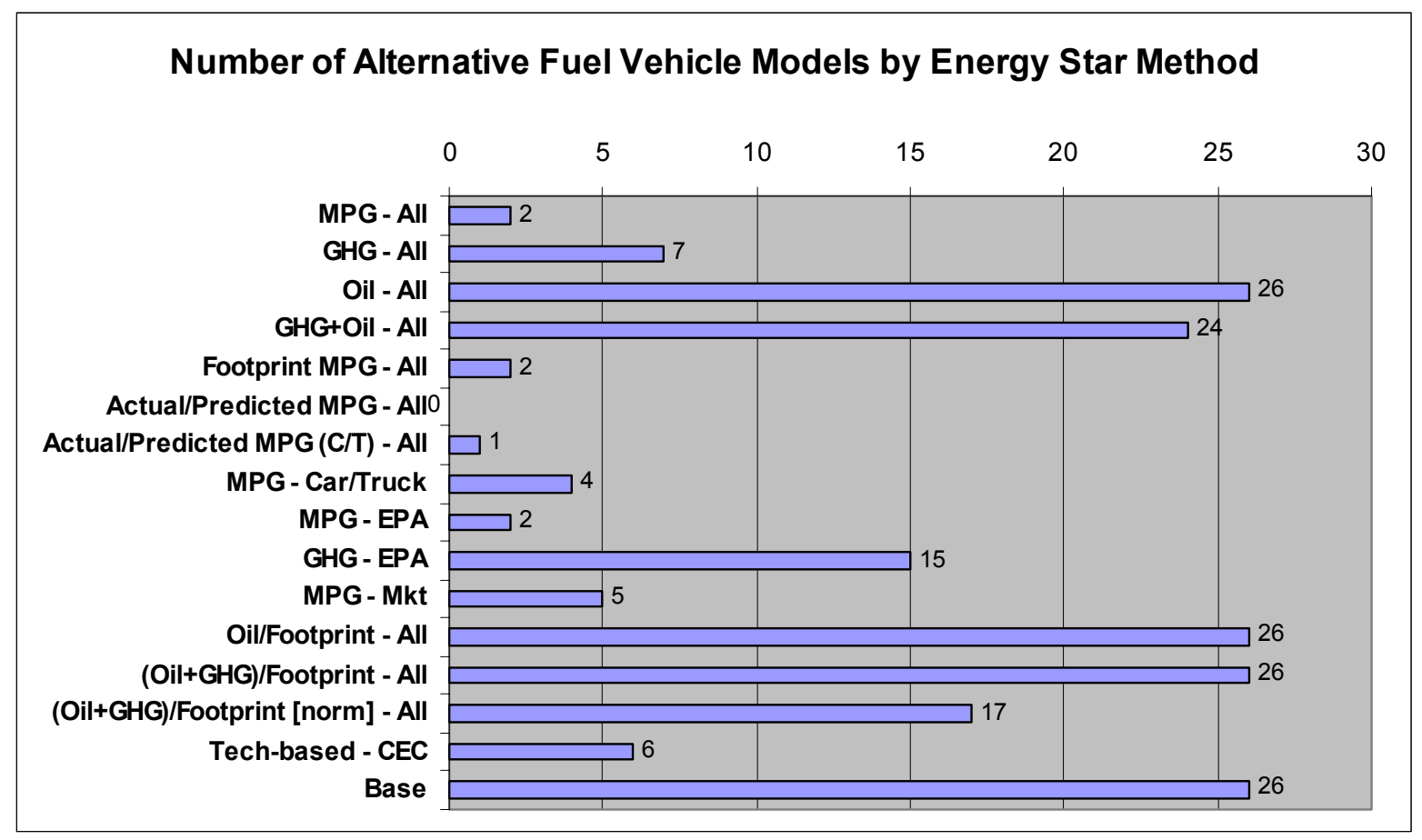

Figure 5. Number of Alternative Vehicles Selected by Each Energy Star Method

\subsubsection{Strengths and Limitations of Each Method}

\subsubsection{Method 1: Highest fuel economy (MPG)}

Method 1 selects vehicle models with MPG in the top 20 percent. Vehicles are not divided into vehicle classes, and the chosen metric (MPG) alone does not consider vehicle attributes. As the statistics show, the vehicles selected by this method have the highest average MPG and lowest GHG emissions of the methods in this study; average fuel economy is 40 percent higher than the base case average, and GHG emissions are 27.4 
percent less than average. Oil use by selected vehicles is, on average, fair to good. This method's average oil use for the selected vehicles ranks somewhat lower (seventh) since the MPG metric is somewhat biased against alternative fuel vehicles (alternative fuels contain less energy and have a lower gasoline-equivalent gallon fuel efficiency).

While this method does a good job of selecting vehicles with desirable measures of merit, it scores poorly at selecting vehicles with desirable attributes. Average acceleration time is fair, about 0.7 seconds more than the sales-weighted average. However, it ranks last in average vehicle size and second to last in number of trucks chosen. In addition, this method selects only two alternative fuel vehicles.

\subsubsection{Method 2: Lowest GHG emissions}

Method 2 selects vehicle models with estimated annual GHG emissions in the lowest 20 percent. Vehicles are not divided into vehicle classes, and the chosen metric (MPG) alone does not consider vehicle attributes. On average, this method does well at selecting vehicles with high fuel economy and low GHG emissions and oil use, ranking second for fuel economy, tied for first for low GHG emissions, and sixth for low oil use. However, it rates poorly on vehicle size and acceleration time, and it selects only 12 trucks, all of which are compact pickups and SUVs. Finally, it only chooses a few alternative fuel vehicles ( 7 of the 26 available models).

\subsubsection{Method 3: Least oil use}

Method 3 selects the vehicle models with petroleum use in the bottom 20 percent. Vehicles are not divided into vehicle classes, and the chosen metric (barrels of oil used annually) alone does not consider vehicle service or choice. The group of vehicles selected by this method rank first in low petroleum consumption; on average, these vehicles use 32 percent less petroleum than the average for model year 2000 vehicles. It also selects vehicles that emit significantly less GHGs, about 25.6 percent less than the average vehicle. The average fuel economy for these vehicles is fair, 29.1 percent better than the average for the base case.

Like the previous two methods, this method does rate poorly at selecting vehicles of typical size and acceleration time. Since alternative fuel vehicles use little to no petroleum, it selects all 26 of these vehicles. It does select more trucks than the previous two methods - about 32 percent of the selected vehicles - but this is partly because many of the alternative fuel vehicles are trucks.

\subsubsection{Method 4: Best combined GHG and oil use scores}

Method 4 rates vehicles based on the weighted average of the GHG and oil use scores from the previous two methods. In this study, GHG and oil use were given equal weight in selecting vehicles. As expected, the resulting vehicle statistics were quite similar to those for the previous two methods, but more closely matching those for the oil-based metric.

\subsubsection{Method 5: Best footprint MPG score}

Method 5 considers both fuel economy and vehicle size (represented by footprint). The average MPG for vehicles selected by this method is somewhat lower than the average for Method 1, which considers MPG only. However, the average fuel efficiency is still almost $32 \mathrm{MPG}, 34.1$ percent higher than the average for all vehicles. This method also selects vehicles with GHG emissions and oil use 24 percent less than the base case. In addition to selecting vehicles with desirable measures of merit, this method also selects vehicles of fair size and acceleration power. Average vehicle footprint is 5.6 percent less than the base-case average, and acceleration time is 9.6 percent longer. However, this method selects a relatively small number of trucks, and it selects only two alternative fuel vehicles. 


\subsubsection{Methods 6 \& 7: Best ratio of actual to predicted MPG based on regression analysis}

The vehicles selected by Methods 6 and 7 have the lowest average fuel economy and highest GHG emissions and oil use of all methods in the study. Those selected by Method 7 have an average MPG only 10 percent better than the average for all vehicles, and the average GHG and oil use scores are only about 8 percent better than the base case. Method 6 was somewhat better; average MPG of the selected vehicles was 20 percent better than the base case, and GHG and oil use were roughly 15 percent lower than the base case.

Both methods scored well in terms of average vehicle size and performance. Although it selected only one truck, Method 6 actually selected bigger vehicles with more acceleration power than the base case average. Method 7, which also considered body style, selected more trucks (55 of the 167 selected vehicles were trucks). It also selected more-powerful vehicles than the base case average, and average vehicle size was only about 3 percent less than the average for all vehicle models. These results suggest that the equations produced by the regression analysis may correct too much for horsepower and weight. Although these vehicles are quite energy efficient in terms of the amount of power provided for the amount of fuel used, promoting these highperformance vehicles will not yield significant energy, GHG, or oil use benefits.

In addition, the noticeable difference in the number of trucks selected by these two methods reflects the significant difference in the fuel efficiency of cars and trucks. Without a variable that accounts for this difference, only one truck is selected using Method 6.

\subsubsection{Method 8: Best MPG in each body type class (car vs. truck)}

This method selects vehicles with fuel economy in the top 20 percent of their respective classes, either car or truck. This method selected a vehicle set with a good average MPG, 31.2 percent higher than the base case. Average GHG emissions and oil use were fair for these vehicles, around 23 percent less than the base case for each. The class system used in this method guarantees a good mix of cars and trucks relative to the base case. However, the vehicles selected by this method rank last in terms of average acceleration time and near the bottom in average vehicle size (10 percent smaller than the overall average). In addition, this method chose only four alternative fuel vehicles.

\subsubsection{Method 9: Best MPG in each type-size class}

This method selects vehicles with fuel economy in the top 20 percent of their respective type-size classes. The vehicle set selected by this method scored fair in most assessment categories. It selected a good mix of cars and trucks (ranking first), and average vehicle size was larger than for most other methods (it ranked fifth overall). Average acceleration time was fair, ranking sixth among the methods explored.

In terms of fuel economy, the method also rates "fair," with an average MPG about 20 percent better than the overall average. Decreases in GHG emissions and oil use were modest—about 16 percent for each. In addition, this method chose only two alternative fuel vehicles.

\subsubsection{Method 10: Lowest GHG emissions in each type-size class}

This method selects vehicles with GHG emissions in the bottom 20 percent of their respective type-size classes. Similar to the previous method, the vehicles selected by this method ranked fair in all measures of merit, as well as vehicle size and acceleration time. It selected a good number of trucks, ranking second among all methods. This method chose 15 alternative fuel vehicles. 


\subsubsection{Method 11: Highest MPG in each market class}

This method selects vehicles with fuel economy in the top 20 percent of their respective market classes. The average MPG and GHG scores were fair, while the oil use score was poor. Average vehicle size was poor, ranking ninth, and acceleration time was fair. The number of trucks selected was comparable to those for the method using type-size classes; both were ranked as good for this metric. However, since there are fewer truck classes in the market class system than in the type-size system, this method selected fewer trucks. It also selected fewer alternative fuel vehicles (only five).

\subsubsection{Method 12: Best ratio of oil use to footprint}

This method rates vehicles based on oil use and size, selecting those with an oil-to-footprint ratio in the top 20 percent. It selected vehicles with fair fuel economy (26 percent above the base case) and GHG emissions (24 percent below the base case). Average oil use by the selected vehicles is good, significantly lower (30 percent less) than the base case average. Sacrifices in size and acceleration are moderate, as this method scored fair for both metrics. This method ranks seventh, along with Method 13, in the number of trucks selected (30 models) and selects all 26 alternative fuel models available.

\subsubsection{Methods 13 and 14: Best ratio of GHG and oil use to footprint}

These methods rate vehicles based on GHG emissions, oil use, and size. Both select the vehicles scoring in the top 20 percent for their respective criteria. Method 13 yields the same results as Method 12: selected vehicles scored fair for fuel economy and GHG emissions, and good for oil use. The statistics for size, acceleration, and vehicle mix are also identical; the method scored fair for each. Like Method 12, this method also selects all 26 alternative fuel vehicles.

Method 14, which normalizes GHG and oil use, selects vehicles with significantly different traits than those selected by Method 13. The vehicles selected by this method scored good on all measures of merit. In fact, it ranks third in each category. However, the vehicles selected by this method rank poorly in average size and vehicle mix, and they rank fair in average acceleration time. This method does, however, select a moderate number of alternative fuel vehicles (17 of 26). 
Table 7. Summary of Strengths and Limitations of Each Method

\begin{tabular}{|c|c|c|c|c|c|c|c|c|}
\hline \multicolumn{2}{|c|}{ Method } & \multicolumn{3}{|c|}{ Measures of Merit } & \multicolumn{3}{|c|}{ Vehicle Attributes } & \multirow[b]{2}{*}{$\begin{array}{l}\text { Alt. Fuel } \\
\text { Vehicles }\end{array}$} \\
\hline No. & Description & MPG & GHG & $\begin{array}{l}\text { Oil } \\
\text { Use }\end{array}$ & Size & $\begin{array}{l}\text { Accel. } \\
\text { Time }\end{array}$ & Mix & \\
\hline 1 & MPG - All & + & + & + & - & 0 & - & - \\
\hline 2 & GHG - All & + & + & + & - & 0 & - & - \\
\hline 3 & Oil - All & 0 & + & + & - & 0 & 0 & + \\
\hline 4 & GHG+Oil - All & + & + & + & - & 0 & 0 & + \\
\hline 5 & Footprint $\times$ MPG - All & + & 0 & 0 & 0 & 0 & - & - \\
\hline 6 & Regress - All & 0 & 0 & - & 0 & + & - & - \\
\hline 7 & Regress $(\mathrm{C} / \mathrm{T})-\mathrm{All}$ & - & - & - & + & + & + & - \\
\hline 8 & MPG - Car/Trk & + & 0 & 0 & - & 0 & + & - \\
\hline 9 & MPG - Type-Size Class & 0 & 0 & 0 & 0 & 0 & + & - \\
\hline 10 & GHG - Type-Size Class & 0 & 0 & 0 & 0 & 0 & + & 0 \\
\hline 11 & MPG - Mkt Class & 0 & 0 & - & - & 0 & + & - \\
\hline 12 & Oil/Footprint - All & 0 & 0 & + & 0 & 0 & 0 & + \\
\hline 13 & (GHG+Oil)/Footprint - All & 0 & 0 & + & 0 & 0 & 0 & + \\
\hline 14 & $\begin{array}{l}\text { (GHG+Oil)/Footprint }- \text { All } \\
\text { [Normalized] }\end{array}$ & + & + & + & - & 0 & - & 0 \\
\hline $\mathrm{T}$ & Technology-based - All & - & - & - & + & + & 0 & - \\
\hline
\end{tabular}

Key: Good (+), Fair (0), Poor (-)

\subsection{GENERAL OBSERVATIONS}

The results suggest that several of the outcome-based methods explored in this study could be used as a basis for an Energy Star rating system for cars and trucks. Several of the methods explored in this study yield reasonable tradeoffs between selecting vehicles with energy and environmental benefits and selecting those that offer diversity in size, performance, and body style. It also shows that methods that only consider fuel economy, GHG, and/or oil use do a poor job of retaining vehicle diversity and consumer choice.

Methods 5 and 8-14 offered reasonable trade-offs between improved measures of merit and sacrifices in vehicle attributes. Since each places emphasis on different measures of merit and vehicle attributes, judgment would have to be made as to the kind of tradeoff that would best prompt a change in consumer and manufacturer behavior.

While Methods 6 and 7 selected a group of vehicles with average MPG, GHG, and oil use that were only modestly better than the base case, this method could be improved by limiting the coefficient for horsepower in the regression equation.

Methods 1 through 4 selected a group of vehicles with good average MPG, GHG, and oil use scores. However, these methods did a poor job of selecting a group of vehicles with diversity of size, performance, 
and body type. This shows that methods using measures of merit alone are unlikely to result in a group of vehicles that sufficiently retain vehicle diversity and consumer choice.

The results from this study suggest a difference between the fuel economies of light trucks and passenger cars of the same size, weight, and horsepower. For the methods explored in this study, those where trucks compete only against other trucks (class-based methods) or where trucks are given special consideration (such as the regression equation that corrects for the lower efficiency of trucks) are the only methods that select a significant number of these vehicles. This relative inefficiency is not solely a result of the heavier weight of trucks, since the regression analysis of Method 6 indicates that trucks are less efficient even when weight and horsepower are considered. This raises an important issue regarding the use of Energy Star for vehicles. Should concessions be given to trucks in order to provide choice and/or increased service or should Energy Star be used to encourage light truck manufacturers to increase the efficiency of these vehicles?

Another notable result concerns alternative fuel vehicles. Only those methods that specifically include oil use in their criteria select a significant number of alternative fuel vehicles. Therefore, unless oil use is explicitly incorporated into an Energy Star criterion, alternative fuel vehicles are likely to be overlooked. Measures combining oil use and GHG emissions also select AFVs. This result is also dependent upon the precise method for calculating oil use. 



\section{TECHNOLOGY-BASED CRITERIA}

\subsection{POTENTIAL APPROACHES}

As previously discussed in this report, an Energy Star system could be based on a vehicle's use of advanced fuel economy technology. For a given set of attributes of size, performance, and features, a vehicle's fuel economy is determined by the technology it uses. There are several issues related to developing a technologybased Energy Star index.

1. Although the technologies that improve fuel economy are well known, complete information on the use of each technology in each vehicle model is not available. However, manufacturers might be willing to provide this information to secure an Energy Star rating.

2. Each vehicle model incorporates "bundles" of technologies that are a subset of identified technologies for fuel efficiency. A comparative index for all cars must weight the contribution of different technologies to fuel efficiency.

3. Fuel economy technologies can usually be used to provide higher levels of performance instead of fuel economy, and so technological characteristics may not be as closely correlated with the measures of merit as desired.

These issues are addressed below.

\subsubsection{Technology Information}

Technologies that improve fuel economy have been studied extensively. A list of current technologies drawn from several recent studies is presented in Table $8 .^{7}$ This list is illustrative rather than comprehensive.

As can be seen from the table, information on a few variables is typically not publicly available, although the situation could change if manufacturers found it in their interest to provide such information in order to obtain an Energy Star rating (i.e., the information is compiled but not published by manufacturers). Two significant variables, tire rolling resistance and engine internal friction, fall in this category, while an aerodynamic drag coefficient is available for many but not all vehicles. For some technologies, such as reduction of power to accessories, or torque converter efficiency, no benchmark level for comparison exists, and the extent of variation among models and manufacturers is not known. However, anecdotal data suggests that these technologies do not have large effects on fuel economy and are unlikely to be major determinants of an Energy Star rating scheme.

\footnotetext{
${ }^{7}$ Energy and Environmental Analysis. 1993. Documentation of Attributes of Technologies to Improve Automotive Fuel Economy. Arlington, Virginia, February.
} 
Table 8. Vehicle Technology Impacts and Data Availability

\begin{tabular}{|c|c|c|c|}
\hline Technology & $\begin{array}{c}\text { Fuel Economy } \\
\text { Improvement }(\%)\end{array}$ & $\begin{array}{l}\text { Information } \\
\text { Availability }\end{array}$ & Comments \\
\hline Weight reduction & $\begin{array}{l}6.6 \% \text { per } 10 \% \\
\text { reduction in weight }\end{array}$ & Indirect & $\begin{array}{l}\text { Computed from } \\
\text { weight/volume ratio }\end{array}$ \\
\hline Drag coefficient & $\begin{array}{l}2.2 \% \text { per } 10 \% \\
\text { reduction in drag }\end{array}$ & $\begin{array}{c}\text { Not } \\
\text { uniformly } \\
\text { available }\end{array}$ & \\
\hline Tire coefficient & $\begin{array}{l}1.8 \% \text { per } 10 \% \\
\text { reduction in rolling } \\
\text { resistance }\end{array}$ & No & \\
\hline \multicolumn{4}{|c|}{ Engine Technologies } \\
\hline 4-valve & $5 \%$ & Yes & \\
\hline Variable valve timing (VVT) & $2 \%$ & Yes & \\
\hline Variable valve timing + lift & $6 \%$ & Yes & \\
\hline Friction reduction & $\begin{array}{c}2 \% \text { per } 10 \% \text { reduction } \\
\text { in friction }\end{array}$ & No & $\begin{array}{l}\text { Auto makers have this } \\
\text { information }\end{array}$ \\
\hline $5 \mathrm{~W}-30$ oil & $0.5 \%$ & No & \\
\hline Efficiency engine accessories & $\begin{array}{c}0.2 \% \text { per } 10 \% \\
\text { reduction in power } \\
\text { use }\end{array}$ & No & $\begin{array}{l}\text { No benchmark for } \\
\text { comparison }\end{array}$ \\
\hline Diesel & $40 \%$ & Yes & \\
\hline Hybrid drive-train & $33 \%$ & Yes & \\
\hline \multicolumn{4}{|c|}{ Transmission Technologies } \\
\hline 5-speed automatic & $2.5 \%$ & Yes & \\
\hline $\begin{array}{l}\text { Continuously variable } \\
\text { transmission (CVT) }\end{array}$ & $6.0 \%$ & Yes & \\
\hline Torque converter lockup & $3.0 \%$ & Yes & \\
\hline Efficient converter & $0-2.0 \%$ & No & No benchmark \\
\hline
\end{tabular}

\subsubsection{Multiple Technologies}

Issues related to technology bundles can be solved by constructing an index comprised of a weighted sum of technology contributions. The estimated percent improvements to fuel economy shown in Table 8 are an appropriate set of weights. Since the weights are to establish a comparative scale, issues of modest synergies or negative synergies between technologies need not be considered, and the index can simply be the sum of weights for the technologies in a particular vehicle. Fortunately, combinations of technologies with significant negative synergies are unlikely to be employed in the same vehicle.

\subsubsection{Technology Variation by Market Class}

The class-specific aspects of technology can be significant. For example, the weight efficiency as defined by the weight-to-volume ratio varies significantly by market class and for high-luxury versus low-luxury 
vehicles. Hence, it may be necessary to perform the index computation at a market-class level to avoid spurious results.

\subsubsection{Vehicle Performance}

Lastly, the issue of harnessing technology potential to improve attributes other than fuel economy is a major concern. This has clearly been the case with acceleration performance during the last decade, as manufacturers have used improved engine designs to increase performance, often at the expense of fuel economy. One can adjust for this by adjusting the point score as a function of the horsepower-to-weight or torque-to-weight ratio. Fuel economy typically decreases 2.2 percent per 10 percent increase in torque-toweight ratio. Hence, increases or decreases of this ratio to the average can be adjusted for easily. However, making such an adjustment requires specifying a reference performance level and implies that higher performance levels are in some sense appropriate.

Sub-optimal applications of technology can also occur in the marketplace. However, earlier statistical analyses and tracking of vehicle fuel economy over time shows that there are a relatively small number of such applications and that, over time, sub-optimal applications move closer to the average. However, such applications could result in minor distortions to the ranking scheme.

\subsection{ILLUSTRATIVE ANALYSIS}

\subsubsection{Approach}

For model year 2000 vehicles, a detailed technology index was constructed that includes several of the technologies (weighted by fuel economy effect) listed in Table 8. Vehicles were awarded points for technologies such as variable valve timing, four-valve engine designs, low-drag design, and 5-speed automatic and continuously variable transmissions. Tire rolling resistance, engine friction, engine accessories, and torque converter efficiency were not included. In addition, corrections for the weight/volume ratio, torque/displacement ratio, and torque/weight ratio were made by determining the class average for each variable and determining the percent difference between a specific model in the class and the class average.

The California Energy Commission's (CEC) vehicle classification system was used for this method. Within each CEC class, vehicles were also classified as "high luxury" or "low luxury." High luxury vehicles were defined as those with a price 30 percent higher than the class average. The percent differences were then weighted for fuel economy effects using the values listed in Table 8 . When the drag co-efficient for a particular model was unavailable, the drag credit was set to zero. The equation used to compute the technology index for each vehicle is as follows:

$$
\begin{aligned}
\text { Index } & \left.=\left(\left\{\left[\frac{\left(\frac{T r q}{C I D}\right)_{v m}}{\overline{\left(\frac{T r q}{C I D}\right)_{\text {class }}}}\right]-1\right\} \times \alpha\right)+\left(\left\{\frac{\left(\frac{T r q}{W t}\right)_{v m}}{\overline{\left(\frac{T r q}{W}\right)_{\text {class }}}}\right]-1\right\} \times \beta\right)+\left\{\left[\frac{\left(\frac{W t}{P v o l}\right)_{v m}}{\left.\left.\left.\overline{\left(\frac{W t}{P v o l}\right)_{\text {class }}}\right]-1\right\} \times \delta\right)}\right.\right. \\
& +4 \text { Val }+V V T+\text { Trans }+ \text { Drag }
\end{aligned}
$$

where

$\left(\frac{T r q}{C I D}\right)_{v m}=$ Ratio of engine torque to engine displacement for the vehicle model; $\operatorname{Trq}=$ torque in Newton meters $(\mathrm{Nm}) ; \mathrm{CID}=$ engine displacement in cubic inches (cu. in.); vm = vehicle 
model

$\overline{\left(\frac{\operatorname{Trq}}{C I D}\right)_{\text {class }}}=$ Average ratio of engine torque to engine displacement for that vehicle model's class
$\left(\frac{\operatorname{Trq}}{W t}\right)_{v m}=$ Ratio of engine torque to vehicle curb weight for the vehicle model; Trq $=$ torque in Newton meters $(\mathrm{Nm}) ; \mathrm{Wt}=$ vehicle curb weight in pounds $(\mathrm{lbs}) ; \mathrm{vm}=$ vehicle model

$\overline{\left(\frac{\operatorname{Trq}}{W t}\right)_{\text {class }}}=$ Average ratio of engine torque to vehicle curb weight for that vehicle model's class

$\left(\frac{W t}{P v o l}\right)_{v m}=$ Ratio of vehicle curb weight for the vehicle model; $\mathrm{Wt}=$ vehicle curb weight in pounds (lbs); Pvol = passenger volume in cubic inches (cu. in.); vm = vehicle model

$\overline{\left(\frac{W t}{P v o l}\right)_{\text {class }}}=$ Average ratio of vehicle curb weight to passenger volume for that vehicle model's class

$\alpha=$ Factor adjusting for the difference between a vehicle's ratio of torque to engine displacement compared to the average for its class (25)

$\beta=$ Factor adjusting for the difference between a vehicle's ratio of torque to curb weight compared to the average for its class $(-22)$

$\delta=\quad=\quad$ Factor adjusting for the difference between a vehicle's ratio of curb weight to passenger volume compared to the average for its class (-66)

$4 \mathrm{Val}=$ Technology factor for having a four-valve engine (5)

VVT $=$ Technology factor for having variable valve timing; 2 for VVT, 6 for VVT and lift

Trans $=$ Transmission technology factor; 2.5 for five-speed automatic, 4 for six-speed automatic, 6 for continuously variable transmission

Drag $=$ Technology factor for coefficient of drag (varies by coefficient of drag)

The vehicles scoring in the top 20 percent of their respective CEC vehicle classes were chosen as Energy Star vehicles.

\subsubsection{Results}

The technology-based method used in this study selected vehicles with poor average measures of merit. Average fuel economy was only 10 percent higher than the average for all vehicles; average GHG emissions were only 8.9 percent lower than the base case average; and average oil use was only 8.6 percent lower. The method did well, however, at selecting a set of vehicles of good size and with good acceleration, ranking third for both measures. It did not necessarily select a good mix of body types, at least in terms of the number of trucks. Only 12 percent of the selected vehicles were trucks since there were about half as many truck classes as car classes. It also selected only six alternative fuel vehicles.

A list of the vehicles selected by this method is provided in Appendix B. 


\section{CONCLUSIONS}

The authors of this report have developed a framework for formulating and evaluating Energy Star ratings for passenger cars and light trucks. This framework is comprised of energy- and environmental-based metrics (measures of merit), potential vehicle classification systems, vehicle technology factors, and vehicle selection criteria. Several concepts and Energy Star rating methods have been tested using model-year 2000 vehicle data, and a spreadsheet model has been developed to facilitate these analyses. This study tests two primary types of rating systems: one that is outcome-based (vehicles are rated based on fuel economy, GHG emissions, and oil use) and another that is technology-based (vehicles are rated based on the energy-saving technologies they use). The methods tested in this study were evaluated based on their ability to select vehicles with high measures of merit (i.e., high fuel economy, low GHG emissions, low oil use) while still preserving a full range of service (size and acceleration) and body style choice.

Results suggest that several methods could be used to achieve reasonable tradeoffs between selecting vehicles with low energy use and emissions and selecting vehicles that represent reasonable diversity in size, performance, and body type. The results also show that any method that considers only fuel economy, GHG emissions, and oil use will not select a diverse mix of vehicles. Analysis suggests that reasonable trade-offs can be achieved by using a method that considers GHG emissions and oil use along with some metric representing vehicle diversity, such as class, size, or performance.

Analysis of technology-based measures suggest that encouraging the use of technology only, may result in increases in acceleration power and weight rather than reductions in oil use and GHG emissions and improvements in fuel economy.

The analysis also suggests that trucks achieve generally poor fuel economy, even when their horsepower and weight are considered. The data also suggest that these vehicles are less likely to use energy-efficient technologies.

Test results also suggest that the only way to select alternative fuel vehicles using one of the outcome-based methods is to explicitly include a metric that considers oil use.

This study concludes that an Energy Star rating for passenger cars and light trucks is feasible. Any decision to implement an Energy Star system for highway vehicles, however, would involve broader consultation and consensus-building as well as additional analysis. Furthermore, including non-energy-related product quality and performance criteria, which were beyond the scope of this study, should be explored. Additional criteria could require that vehicles meet specified safety, crash test, warranty, and/or emissions standards, or any other criteria that would ensure the Energy Star label is only awarded to quality products that are in keeping with Energy Star's market image. 


\section{REFERENCES}

Argonne National Laboratory. 2002. The Greenhouse Gases, Regulated Emissions, and Energy Use in Transportation (GREET) Model (http://greet.anl.gov/default.htm).

Argonne National Laboratory. GREET Model, (http://www.transportation.anl.gov/ttrdc/greet/index.html).

DeCicco, J. and J. Kliesch. 2001. ACEEE's Green Book: The Environmental Guide to Cars and TrucksModel Year 2001. American Council for an Energy-Efficient Economy, Washington, DC.

U.S. Department of Energy, Office of Energy Efficiency and Renewable Energy. 2000. 10 CFR 474: Electric and Hybrid Vehicle Research, Development, and Demonstration Program; PetroleumEquivalent Fuel Economy Calculation; Final Rule. Federal Register, Vol. 65, No. 113, June 12, pp. 36986-36992.

(EEA) Energy and Environmental Analysis, Inc. 2002. Memo to Oak Ridge National Laboratory, November 25.

(EEA) Energy and Environmental Analysis. 1993. Documentation of Attributes of Technologies to Improve Automotive Fuel Economy. Arlington, Virginia, February.

(EPA) Environmental Protection Agency. 2001. Join Us In Making A Change. (Energy Star Fact Sheet) (http://www.epa.gov/nrgystar/newsroom/pdf/estarchange9.pdf).

(EPA) Environmental Protection Agency. Energy Star Program Requirements for Programmable Thermostats. (http://estar4.energystar.gov/estar/espartnerlogos.nsf/ pdf/files/\$file/program.pdf).

(EPA) Environmental Protection Agency. Energy Star Program Requirements for Residential Air-Source Heat Pumps (ASHPs) and Central Air Conditioners. (http://134.67.99.43/

Estar/consumers.nsf/attachments/HVACSpec2.pdf/\$File/ HVACSpec2.pdf?OpenElement).

(EPA) Environmental Protection Agency. Energy Star Program Requirements for Consumer Audio and DVD Products. (http://estar4.energystar.gov/estar/espartnerlogos.nsf/pdf/files/\$file/ audiodvdprogreq.pdf).

(EPA) Environmental Protection Agency. Energy Star Specifications: Appliances. (http://www.energystar.gov/products/ appliancespecs.pdf).

(EPA) Environmental Protection Agency. Energy Star Labeled Computers and Monitors: Key Product Criteria. (http://www.epa.gov/nrgystar/purchasing/6a_c\&m.html\#specs_cm).

(EPA) Environmental Protection Agency. 1994. Fuel Economy Test Procedures Alternative-Fueled Automobile CAFE Incentives and Fuel Economy Labeling Requirements; Final Rule. Federal Register, Vol. 59, August 3.

Sanchez, M. C.; Clark, R.; Fanara, A.; Hershbert, C.; and Schmeltz, R. 2002. New Product Development: The Pipeline for Future Energy Star Growth. ACEEE Summer Study on Energy Efficiency in Buildings. 
United States Code. Title 49, Sec. 32905 (http://frwebgate4.access.gpo.gov/cgibin/waisgate.cgi?WAISdocID $=14776724133+0+0+0 \&$ WAISaction=retrieve) 
APPENDIX A

\section{ALTERNATIVE EFFICIENCY-BASED APPROACH TO RANKING VEHICLES}





\section{APPENDIX A. ALTERNATIVE EFFICIENCY-BASED APPROACH TO RANKING VEHICLES}

Energy and Environmental Analysis, Inc., has explored several alternative methods for ranking the energy efficiency of vehicles. ${ }^{8}$ These methods use the California Energy Commission's (CEC) interior-volume-based classification scheme to disaggregate vehicles into different groups. This classification system differs from the EPA system in two ways: (1) the EPA system uses a volume definition that includes trunk volume, which results in hatchbacks being classified with larger cars, and (2) the type-size classification system for trucks is not size-based. The CEC system has 6 classes of cars and 7 classes of light trucks. Cars, vans, and SUVs are classified based on passenger volume, while pickups are classified based on Gross Vehicle Weight Rating (GVWR) and drive system (four-wheel drive vs. two-wheel drive).

Table A1. CEC Vehicle Classifications

\begin{tabular}{llcc}
\hline CEC Class & Description & $\begin{array}{c}\text { Passenger Volume } \\
(\mathrm{Cu} . \text { Ft. })\end{array}$ & $\begin{array}{c}\text { Gross Vehicle } \\
\text { Weight Rating (lbs. })\end{array}$ \\
\hline 1 & Mini & $<82$ & - \\
\hline 2 & Sub Compact & $82-85$ & - \\
\hline 3 & Compact & $85-95$ & - \\
\hline 4 & Mid Size & $95-105$ & - \\
\hline 5 & Large & $106+$ & - \\
\hline 6 & Sports & $-89(2$ seats $)$ & - \\
\hline 7 & Compact Pickup & & 4,250 for 2WD \\
\hline 8 & & - & $\leq, 500$ for 4WD \\
\hline 9 & Standard Pickup & & $>4,250$ for 2WD \\
\hline 10 & & $\leq 200$ & - \\
\hline 11 & Compact Van & $>200$ & - \\
\hline 12 & Standard Van & $121-160$ & - \\
\hline 13 & Compact SUV & $>160$ & - \\
\hline & Standard SUV & $\leq 120$ & for 4WD \\
\hline
\end{tabular}

CEC car classes are further categorized into "high luxury" and "low luxury" classes, using price as an indicator for luxury. Vehicles with a retail price 30 percent higher than the sales-weighted average for their size class are classified as luxury vehicles; others are classified as non-luxury. For example, the Mercedes Benz CLK 320 (120 percent above class average) is classified as a luxury vehicle, while the Volkswagen Cabrio (24 percent above class average) is classified as non-luxury. This price-based criterion is not used for trucks (i.e., pickups, vans, and SUVs).

$8 \quad$ Energy and Environmental Analysis, Inc. 2002. Memo to Oak Ridge National Laboratory, November 25. 
Table 2 lists the fuel economy statistics for each class, showing that there is considerable variation among the classes. The last column lists the coefficient of variation, equal to the standard deviation as a percentage of the mean. A low coefficient indicates low variation within that class, which makes it difficult to have a distinction within each class based on MPG. In order to account for the fact that many of the classes do have low coefficients of variation, the following analysis treats volume as a continuous variable while also incorporating variables to account for performance. This provides criteria to distinguish vehicles that are significantly better than the average.

Table A2. Fuel Economy Indicators for Each Class

\begin{tabular}{|c|c|c|c|c|c|}
\hline Class & Luxury & $\begin{array}{l}\text { 10th } \\
\text { Percentile } \\
\text { (MPG) }\end{array}$ & $\begin{array}{c}\text { Mean } \\
\text { (MPG) }\end{array}$ & $\begin{array}{c}\text { 90th } \\
\text { Percentile } \\
\text { (MPG) }\end{array}$ & $\begin{array}{c}\text { Coefficient of } \\
\text { Variation } \\
(\%)\end{array}$ \\
\hline \multicolumn{6}{|l|}{ Cars } \\
\hline Mini & High & 15.3 & 21.0 & 27.5 & 37 \\
\hline Mini & Low & 31.3 & 38.2 & 47.9 & 19 \\
\hline Subcompact & High & 23.2 & 26.3 & 29.3 & 9 \\
\hline Subcompact & Low & 27.2 & 30.9 & 38.6 & 28 \\
\hline Compact & High & 21.1 & 24.5 & 28.4 & 26 \\
\hline Compact & Low & 26.3 & 30.4 & 36.1 & 21 \\
\hline Inter. & High & 20.9 & 22.9 & 26.5 & 21 \\
\hline Inter. & Low & 24.4 & 27.0 & 30.3 & 8 \\
\hline Large & High & 18.1 & 20.8 & 23.5 & 15 \\
\hline Large & Low & 23.7 & 25.9 & 27.6 & 5 \\
\hline Sports & High & 17.4 & 22.2 & 26.0 & 18 \\
\hline Sports & Low & 17.0 & 25.9 & 35.2 & 34 \\
\hline \multicolumn{6}{|l|}{ Trucks } \\
\hline Compact Pickup & & 19.1 & 22.5 & 27.5 & 15 \\
\hline Large Pickup & & 16.8 & 19.2 & 20.9 & 8 \\
\hline Compact Van & & 20.0 & 22.6 & 25.0 & 8 \\
\hline Large Van & & 16.5 & 17.6 & 18.7 & 5 \\
\hline Compact SUV & & 18.7 & 20.9 & 23.9 & 11 \\
\hline Large SUV & & 16.3 & 17.5 & 18.6 & 6 \\
\hline Mini SUV & & 22.6 & 26.7 & 30.7 & 12 \\
\hline
\end{tabular}

The above table indicates significantly more fuel economy variation in the car classes than in the truck classes, and more variation in the luxury classes than in the standard classes. Hence, the identification of Energy Star vehicles clearly favors some classes of vehicles if defined with respect to the class average. 


\section{INCORPORATING OTHER ATTRIBUTES}

Incorporating other vehicle attributes into the analysis suggests alternative criteria while simultaneously giving a point of comparison for the criterion using simple MPG by class. For these purposes, EEA has developed regressions with variables that consider performance and weight or performance and volume.9 Two separate regressions were utilized:

$F E=\left(\frac{\text { Torq }}{W t}\right)^{\alpha} \times \operatorname{Pvol}^{\beta} \times e^{(\delta \times 4 W D)} \times e^{(\varepsilon \times M \text { trans })}$

where

$\mathrm{FE}=$ fuel economy in miles/gallon (MPG)

Torq $=$ torque in Newton meters $(\mathrm{Nm})$

$\mathrm{Wt}=$ vehicle test weight in pounds (lbs)

Pvol $=$ passenger volume in cubic feet (cu. Ft.)

$4 \mathrm{WD}=$ dummy variable indicating whether or not the vehicle has four-wheel drive $(0=$ no; $1=$ yes $)$

Mtrans = dummy variable indicating whether or not the vehicle has a manual transmission $(0=$ no; $1=$ yes $)$

$\alpha=$ coefficient for torque-to-weight ratio

$\beta=$ coefficient for passenger volume

$\delta=$ coefficient for four-wheel drive vehicles

$\varepsilon=$ coefficient for vehicles with manual transmission

$F E=\left(\frac{H p}{W t}\right)^{\alpha} \times W t^{\beta} \times e^{(\delta \times 4 W D)} \times e^{(\varepsilon \times M \text { trans })}$

where

$\mathrm{FE}=$ fuel economy in miles/gallon (MPG)

$\mathrm{Hp}=$ engine power in horsepower

$\mathrm{Wt}=$ vehicle test weight in pounds (lbs)

Pvol = passenger volume in cubic feet (cu. Ft.)

9 Energy and Environmental Analysis, Inc. 2002. Memo to Oak Ridge National Laboratory, November 25. 
$4 \mathrm{WD}=$ dummy variable indicating whether or not the vehicle has four-wheel drive $(0=$ no; $1=$ yes $)$

Mtrans $=$ dummy variable indicating whether or not the vehicle has a manual transmission $(0=$ no; $1=$ yes $)$

$\alpha=$ coefficient for horsepower-to-weight ratio

$\beta=$ coefficient for passenger volume

$\delta=$ coefficient for four-wheel drive vehicles

$\varepsilon=$ coefficient for vehicles with manual transmission

All of the regressions were in log space and were sales-weighted to reduce the effect of special niche market vehicles. This weighting has the effect of reducing luxury cars' impact on the computed coefficients. The reasons for using these variables are as follows:

- Both the horsepower-to-weight ratio and the torque-to-weight ratio are used as performance indicators. The higher the ratio, the higher the performance. Horsepower is generally correlated with torque. However, torque is a more accurate indicator for low-speed towing performance, while horsepower is a more useful indicator of high-speed acceleration.

- Adding extra features or increasing the size of the vehicle will tend to increase the weight of the vehicle. Therefore, "test weight" is used as a composite surrogate for luxury and size. Test weight is the weight to which the dynamometer is set when vehicles are tested for fuel economy.

- Volume is a direct measure of the size of the vehicle, a desirable characteristic with a negative impact on fuel economy.

- Four-wheel drive (4WD) is an increasingly common attribute that does come with a fuel economy penalty. A dummy variable is used here to control for the negative fuel economy impact of this technology.

- Manual transmissions comprise a small percentage of the light-duty vehicle (LDV) market; most consumers purchase automatics and will continue to do so. A dummy variable is used here to control for the fuel economy benefits of this technology.

If either dummy variable was statistically insignificant, it was removed from the regression. The regression coefficients used for the analysis are shown in Tables A3 and A4.

Using these regressions, the predicted value of fuel economy for each car and truck was computed, as was the difference between the predicted and actual fuel economy. A positive value indicates that the vehicle's actual fuel economy is better than would be predicted, given its physical characteristics. 
Table A3. Weight-based Regression Results

\begin{tabular}{|c|c|c|c|c|}
\hline \multirow[b]{2}{*}{ Variable } & \multicolumn{2}{|c|}{ Cars } & \multicolumn{2}{|c|}{ Trucks } \\
\hline & $\begin{array}{l}\text { Regression } \\
\text { Coefficient }\end{array}$ & $\mathrm{T}$ - Statistic & $\begin{array}{l}\text { Regression } \\
\text { Coefficient }\end{array}$ & $\mathrm{T}$ - Statistic \\
\hline $\log (\mathrm{HP} / \mathrm{Wt})$ & -0.316 & -18.10 & -0.176 & -6.95 \\
\hline Log (Weight) & -0.720 & -24.90 & -0.764 & -33.36 \\
\hline 4-Wheel Drive & NS & & -0.014 & -4.26 \\
\hline Manual Transmission & 0.008 & 2.20 & NS & \\
\hline
\end{tabular}

Dependent Variable $=$ Log (Fuel Economy)

$\mathrm{NS}=$ not significant

Table A4. Volume-based Regression Results

\begin{tabular}{l|cc|cr}
\hline \multirow{2}{*}{ Variable } & \multicolumn{2}{|c|}{ Cars } & \multicolumn{2}{c}{ Trucks } \\
& $\begin{array}{c}\text { Regression } \\
\text { Coefficient }\end{array}$ & T - Statistic & $\begin{array}{c}\text { Regression } \\
\text { Coefficient }\end{array}$ & T - Statistic \\
\hline Log (Torque/Wt) & -0.581 & -27.82 & -0.472 & -11.01 \\
Log (Volume) & -0.080 & -2.69 & -0.249 & -10.91 \\
4-Wheel Drive & -0.029 & -3.77 & -0.036 & -5.87 \\
Manual Transmission & NS & & \multicolumn{2}{|c|}{ NS } \\
\hline
\end{tabular}

Dependent Variable $=$ Log (Fuel Economy)

$\mathrm{NS}=$ not significant

Three ranking exercises were performed. The first method ranks all vehicles, by class, in descending order of MPG. The second is based on the difference between the actual MPG and the MPG predicted using the volume-based regression equation. The third is based on the difference between the actual MPG and the MPG predicted using the weight-based regression equation. This facilitates a comparison between the rankings produced from using just MPG and those produced when physical attributes are considered. Rather than comparing the complete rankings, the top ranking vehicles that accounted for at least 15 percent of their class' sales were compared. The table below lists the rankings resulting from these methods, but with all low-sales volume vehicles removed (where low-sales is defined as comprising less than 5 percent of the class' sales). " $\mathrm{M}$ " indicates an automatic transmission, and "(4)" indicates a 4-wheel drive. 
Table A5. Ranking Comparison with Low-Sales Vehicles Removed

\begin{tabular}{|c|c|c|c|c|c|}
\hline \multirow[b]{2}{*}{ Class } & \multirow[b]{2}{*}{$\begin{array}{c}\text { In } \\
\text { All? }\end{array}$} & \multirow[b]{2}{*}{ Model } & \multicolumn{3}{|c|}{ Ranking Method } \\
\hline & & & MPG & $\begin{array}{l}\text { Volume-Based } \\
\text { Residual }\end{array}$ & $\begin{array}{c}\text { Weight-Based } \\
\text { Residual }\end{array}$ \\
\hline $1 \mathrm{~h}$ & $\mathrm{Y}$ & Mer-Benz CLK 320 & $\mathrm{X}$ & $\mathrm{X}$ & $\mathrm{X}$ \\
\hline $1 \mathrm{~h}$ & & Saab Saab 9-3 Cvt M & $\mathrm{X}$ & & \\
\hline 11 & $\mathrm{Y}$ & Toyota Echo M & $\mathrm{X}$ & $\mathrm{X}$ & $\mathrm{X}$ \\
\hline 11 & Y & Toyota Echo & $X$ & $X$ & $X$ \\
\hline $2 \mathrm{~h}$ & & Audi A4 Quattro M (4) & $X$ & & $X$ \\
\hline $2 \mathrm{~h}$ & & Volvo S40 & $\mathrm{X}$ & $\mathrm{X}$ & \\
\hline $2 \mathrm{~h}$ & & Volvo V40 & & & $\mathrm{X}$ \\
\hline 21 & $\mathrm{Y}$ & Toyota Corolla & $\mathrm{X}$ & $\mathrm{X}$ & $\mathrm{X}$ \\
\hline 21 & & Honda Civic Ex & $\mathrm{X}$ & & $\mathrm{X}$ \\
\hline $3 \mathrm{~h}$ & $\mathrm{Y}$ & Mer-Benz C 230 & $\mathrm{X}$ & $\mathrm{X}$ & $\mathrm{X}$ \\
\hline $3 \mathrm{~h}$ & $\mathrm{Y}$ & Acura $3.2 \mathrm{tl}$ & $X$ & $\mathrm{X}$ & $\mathrm{X}$ \\
\hline 31 & $\mathrm{Y}$ & Honda Civic Dx 4dr & $\mathrm{X}$ & $\mathrm{X}$ & $\mathrm{X}$ \\
\hline 31 & & Dodge Neon & $\mathrm{X}$ & $\mathrm{X}$ & \\
\hline \multirow[t]{2}{*}{31} & & Pontiac Grand Am Gt & & $\mathrm{X}$ & \\
\hline & & Pontiac Grand Am & & & $\mathrm{X}$ \\
\hline 31 & & Chevy Cavalier & $\mathrm{X}$ & & \\
\hline $4 \mathrm{~h}$ & Y & Mer-Benz E 320 & $X$ & $X$ & $\mathrm{X}$ \\
\hline $4 h$ & $\mathrm{Y}$ & Infinity I30 & $\mathrm{X}$ & $\mathrm{X}$ & $\mathrm{X}$ \\
\hline $4 h$ & $\mathrm{Y}$ & Cadillac Seville & $\mathrm{X}$ & $\mathrm{X}$ & $X$ \\
\hline 41 & & Nissan Maxima & & $\mathrm{X}$ & $\mathrm{X}$ \\
\hline 41 & & Toyota Camry & $X$ & & \\
\hline 41 & & Honda Accord Lx & $\mathrm{X}$ & & $\mathrm{X}$ \\
\hline 41 & & Chevy Malibu & & $\mathrm{X}$ & \\
\hline 41 & & Honda Accord Coupe & & $\mathrm{X}$ & $X$ \\
\hline $5 \mathrm{~h}$ & Y & Cadillac Deville & $X$ & $\mathrm{X}$ & $\mathrm{X}$ \\
\hline 51 & $\mathrm{Y}$ & Toyota Avalon & $\mathrm{X}$ & $\mathrm{X}$ & $\mathrm{X}$ \\
\hline 51 & & Chevy Impala & $\mathrm{X}$ & $\mathrm{X}$ & \\
\hline 51 & & Chrysler $300 \mathrm{M}$ & & & $X$ \\
\hline $6 \mathrm{~h}$ & & Chevy Corvette M & & $\mathrm{X}$ & $\mathrm{X}$ \\
\hline $6 \mathrm{~h}$ & & BMW Z3 $2.5 \mathrm{M}$ & $X$ & & \\
\hline
\end{tabular}




\begin{tabular}{|c|c|c|c|c|c|}
\hline \multirow[b]{2}{*}{ Class } & \multirow[b]{2}{*}{$\begin{array}{c}\text { In } \\
\text { All? }\end{array}$} & \multirow[b]{2}{*}{ Model } & \multicolumn{3}{|c|}{ Ranking Method } \\
\hline & & & MPG & $\begin{array}{l}\text { Volume-Based } \\
\text { Residual }\end{array}$ & $\begin{array}{l}\text { Weight-Based } \\
\text { Residual }\end{array}$ \\
\hline $6 \mathrm{~h}$ & & Chevy Corvette & & $\mathrm{X}$ & $\mathrm{X}$ \\
\hline $6 \mathrm{~h}$ & & Porsche Boxster M & $\mathrm{X}$ & & \\
\hline $6 \mathrm{~h}$ & & BMW Z3a 2.5 & $\mathrm{X}$ & & \\
\hline 61 & $\mathrm{Y}$ & Toyota Celica & $\mathrm{X}$ & $\mathrm{X}$ & $\mathrm{X}$ \\
\hline 61 & Y & Ford Mustang M & $\mathrm{X}$ & $\mathrm{X}$ & $\mathrm{X}$ \\
\hline 61 & & Mitsubishi Eclipse & & & $\mathrm{X}$ \\
\hline 7 & & Ford Ranger $4 \times 2 \mathrm{M}$ & $\mathrm{X}$ & & $\mathrm{X}$ \\
\hline 7 & & Chevrolet S10 P/U 2wd & $\mathrm{X}$ & & $\mathrm{X}$ \\
\hline 7 & & Dodge Dakota 2wd & & & $\mathrm{X}$ \\
\hline 8 & & Ford F150 4x2 & $\mathrm{X}$ & & $\mathrm{X}$ \\
\hline 8 & & Chevrolet C1500 Silverado 2wd & $\mathrm{X}$ & & $\mathrm{X}$ \\
\hline 8 & & Ford F150 4x4 (4) & & & $\mathrm{X}$ \\
\hline 9 & & Chevrolet Venture & $\mathrm{X}$ & $\mathrm{X}$ & \\
\hline 9 & & Honda Odyssey & & $\mathrm{X}$ & $\mathrm{X}$ \\
\hline 9 & & Toyota Sienna & $\mathrm{X}$ & & $\mathrm{X}$ \\
\hline 10 & $\mathrm{Y}$ & Ford E150 Van & $\mathrm{X}$ & $\mathrm{X}$ & $\mathrm{X}$ \\
\hline 10 & $\mathrm{Y}$ & Chevrolet G15/25 Chevy Van & $\mathrm{X}$ & $\mathrm{X}$ & $\mathrm{X}$ \\
\hline 11 & $\mathrm{Y}$ & Chevrolet Blazer (4) & $\mathrm{X}$ & $\mathrm{X}$ & $\mathrm{X}$ \\
\hline 11 & & Ford Explorer 4x4 (4) & & & $\mathrm{X}$ \\
\hline 11 & & Jeep Cherokee (4) & $\mathrm{X}$ & $\mathrm{X}$ & \\
\hline 11 & & Jeep Grand Cherokee (4) & $\mathrm{X}$ & & $\mathrm{X}$ \\
\hline 12 & Y & Chevrolet K1500 Tahoe 4wd (4) & $\mathrm{X}$ & $\mathrm{X}$ & $\mathrm{X}$ \\
\hline 12 & & Ford Expedition $4 \times 2$ & $\mathrm{X}$ & & $\mathrm{X}$ \\
\hline 12 & & Chevrolet K1500 Sub'n 4wd (4) & & $\mathrm{X}$ & \\
\hline 13 & & Chevrolet Tracker Van 4x4 (4) & $\mathrm{X}$ & $\mathrm{X}$ & \\
\hline 13 & & Honda Cr-V & $\mathrm{X}$ & & \\
\hline 13 & $\mathrm{Y}$ & Honda Cr-V (4) & $\mathrm{X}$ & $\mathrm{X}$ & $\mathrm{X}$ \\
\hline
\end{tabular}




\section{FINDINGS}

The above methods were compared to determine whether ranking vehicles based on MPG within vehicle class would produce arbitrary results. However, the findings are indicative rather than conclusive. The different ranking systems give results that are similar for some classes (e.g., $3 \mathrm{H}$ and $4 \mathrm{H}$ ) and very different for others $(6 \mathrm{H})$. The most notable result is that the volume-based rankings are almost identical to the MPG rankings, with the exceptions of classes $4 \mathrm{~L}$ and $6 \mathrm{H}$.

This similarity has two important implications. First, with low-sales vehicles removed, all three ranking systems produce lists of high-technology cars. This is an appropriate result from an energy-efficiency criterion. However, it also raises the issue of whether it is in fact appropriate to control for performance and/or size. The argument can be made that people should be encouraged to use smaller and/or less powerful vehicles as a means of increasing energy-efficiency. Fortunately, this controversy is avoided, as Table 5 indicates that the MPG rankings are similar to the weight- and volume-based rankings.

\section{COMPARING RESIDUALS TO AVERAGE CLASS FUEL ECONOMY}

Another possible approach is to predict each vehicle's fuel economy based on its attributes and compare the residual (i.e., the difference between the actual and predicted fuel economy) to the average fuel economy of its vehicle class. For this approach, EEA has run separate regressions on cars and trucks. The horsepower-toweight ratio was used for cars, while the torque-to-weight ratio was used for trucks. These were used as performance indicators, as discussed previously, with torque being a better indicator for trucks and giving a better fit for the regression. Four-wheel drive, manual transmission, and van are all dummy variables. Van is set equal to 1 if the vehicle is in CEC class 9 or 10. The regression equations are shown below, and the variables and results are shown in the Table A6.

$$
F E_{C a r}=\left(\frac{H p}{W t}\right)^{\alpha} \times W t^{\beta} \times e^{(\delta \times M t r a n s)}
$$

where

$\mathrm{FE}_{\text {Car }}=$ car fuel economy in MPG

$\mathrm{Hp} / \mathrm{Wt}=$ horsepower-to-weight ratio (hp/lb)

$\mathrm{Wt}=$ test weight (pounds)

Mtrans $=$ dummy variable for manual transmission $(0=$ no; $1=y e s)$

$\alpha=$ horsepower-to-weight coefficient

$\beta=$ weight coefficient

$\delta=$ manual transmission coefficient

$F E_{\text {Trk }}=\left(\frac{\text { Torq }}{W t}\right)^{\alpha} \times W t^{\beta} \times e^{(\delta \times 4 W D)} \times e^{(\varepsilon \times V a n)}$

where

$\mathrm{FE}_{\mathrm{Trk}}=$ truck fuel economy in MPG

Torq $/ \mathrm{Wt}=$ torque-to-weight ratio in Newton-meters per pound $(\mathrm{Nm} / \mathrm{lb})$ 
$\mathrm{Wt}=$ vehicle test weight in pounds $(\mathrm{lbs})$

$4 \mathrm{WD}=$ dummy variable indicating whether or not the vehicle has four-wheel drive $(0=$ no;

$1=\mathrm{yes})$

Van $=$ dummy variable indicating whether or not the vehicle is a van $(0=$ no; $1=y e s)$

$\alpha=$ torque/weight coefficient

$\beta=$ weight coefficient

$\delta=4$ WD coefficient

$\varepsilon=$ van coefficient

All of the regressions were in log space and were sales-weighted to reduce the effect of special niche market vehicles. This weighting has the effect of reducing luxury cars' impact on the computed coefficients.

Table A6. Regression Results

\begin{tabular}{l|cc|cc}
\hline & \multicolumn{2}{|c|}{ Cars } & \multicolumn{2}{c}{ Trucks } \\
Variable & $\begin{array}{c}\text { Regression } \\
\text { Coefficient }\end{array}$ & $\mathrm{T}$ - Statistic & $\begin{array}{c}\text { Regression } \\
\text { Coefficient }\end{array}$ & $\mathrm{T}$ - Statistic \\
\hline Log (HP/Wt) & -0.17 & -9.9 & & -8.3 \\
Log (TQ/Wt) & & & -0.22 & -20.5 \\
Log (Weight) & -0.74 & -31.9 & -0.62 & -5.7 \\
4-Wheel Drive & & & -0.02 & \\
Manual Transmission & 0.01 & 2.4 & & 6.2 \\
Van & & & 0.03 & \\
\hline
\end{tabular}

Vehicles were ranked as follows: First, the difference between the predicted and actual MPG was calculated. If the improvement was more than 10 percent of the class average MPG, the vehicle was considered energy efficient.

$\%$ Diff $=\frac{M P G_{\text {Act }}-M P G_{\text {Pred }}}{\overline{M P G_{\text {Class }}}}$

The following table lists vehicles with MPG improvement greater than 10 percent of the class average MPG. In the table, the fuel economy difference is the difference between a vehicle's actual and predicted MPG. Sales percent is that model's sales as a percentage of the sum of all sales for that vehicle's class. 


\begin{tabular}{|c|c|c|c|c|c|c|c|c|c|}
\hline Class & Name & Drive & $\begin{array}{l}\text { Test } \\
\text { Wt. } \\
\text { (lbs) }\end{array}$ & $\begin{array}{l}\text { Horse- } \\
\text { power }\end{array}$ & $\begin{array}{l}\text { Trans- } \\
\text { mission } \\
\text { Type }\end{array}$ & $\begin{array}{l}\text { Fuel } \\
\text { Econ. } \\
\text { (MPG) }\end{array}$ & $\begin{array}{l}\text { Actual } \\
\text { MPG - } \\
\text { Predicted } \\
\text { MPG }\end{array}$ & $\begin{array}{c}\% \\
\text { Diff. } \\
\text { from } \\
\text { Class } \\
\text { Avg } \\
\text { MPG }\end{array}$ & $\begin{array}{l}\text { Sales } \\
\%\end{array}$ \\
\hline $1 \mathrm{H}$ & $\begin{array}{l}\text { Mer-Benz Clk } 320 \\
\text { (Cabriolet) }\end{array}$ & $\mathrm{R}$ & 3,875 & 214 & L5 & 27.5 & 2.6 & 10.9 & 14.1 \\
\hline $1 \mathrm{~L}$ & Chevy Metro & $\mathrm{F}$ & 2,375 & 79 & S5 & 47.5 & 7.8 & 20.5 & 0.8 \\
\hline $1 \mathrm{~L}$ & Chevy Metro & $\mathrm{F}$ & 2,125 & 55 & S5 & 50.8 & 5.9 & 15.4 & 3.0 \\
\hline $1 \mathrm{~L}$ & Toyota Echo & $\mathrm{F}$ & 2,250 & 108 & M5 & 43.1 & 4.3 & 11.1 & 8.5 \\
\hline $2 \mathrm{~L}$ & Honda Civic Hx & $\mathrm{F}$ & 2,625 & 115 & M5 & 44.7 & 9.5 & 29.2 & 0.3 \\
\hline $2 \mathrm{~L}$ & Honda Civic Hx & $\mathrm{F}$ & 2,750 & 115 & $\mathrm{LV}$ & 41.9 & 8.3 & 25.5 & 0.2 \\
\hline $2 \mathrm{~L}$ & Chevy Prizm & $\mathrm{F}$ & 2,750 & 125 & M5 & 41.5 & 7.7 & 23.6 & 0.3 \\
\hline $2 \mathrm{~L}$ & Toyota Corolla & $\mathrm{F}$ & 2,750 & 125 & M5 & 39.6 & 5.8 & 17.8 & 1.5 \\
\hline $2 \mathrm{~L}$ & Chevy Prizm & $\mathrm{F}$ & 2,750 & 125 & E4 & 38.8 & 5.7 & 17.4 & 0.8 \\
\hline $2 \mathrm{~L}$ & Toyota Corolla & $\mathrm{F}$ & 2,750 & 125 & L4 & 37.4 & 4.3 & 13.1 & 12.2 \\
\hline $2 \mathrm{~L}$ & Mitsubishi Mirage 2d & $\mathrm{F}$ & 2,500 & 92 & M5 & 41.5 & 3.9 & 12.1 & 0.5 \\
\hline $2 \mathrm{~L}$ & Honda Civic Ex & $\mathrm{F}$ & 2,750 & 127 & M5 & 37.2 & 3.5 & 10.7 & 4.1 \\
\hline $2 \mathrm{~L}$ & Honda Civic Ex & $\mathrm{F}$ & 2,875 & 127 & L4 & 35.6 & 3.4 & 10.3 & 8.8 \\
\hline $3 \mathrm{~L}$ & Mazda Protege & $\mathrm{F}$ & 2,875 & 122 & L4 & 36.4 & 4.0 & 12.6 & 0.7 \\
\hline $3 \mathrm{~L}$ & Honda Civic Dx 4Dr & $\mathrm{F}$ & 2,625 & 106 & M5 & 39.5 & 3.8 & 12.2 & 1.3 \\
\hline $3 \mathrm{~L}$ & Dodge Neon & $\mathrm{F}$ & 2,875 & 132 & M5 & 36.1 & 3.4 & 10.9 & 1.3 \\
\hline $3 \mathrm{~L}$ & Saturn Sl & $\mathrm{F}$ & 2,850 & 124 & E4 & 35.8 & 3.3 & 10.4 & 1.9 \\
\hline $4 \mathrm{H}$ & 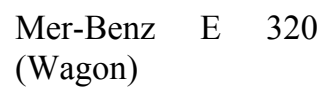 & $\mathrm{R}$ & 4,000 & 221 & L5 & 27.5 & 3.1 & 13.3 & 0.6 \\
\hline $4 \mathrm{H}$ & Mer-Benz E 320 & $\mathrm{R}$ & 3,875 & 221 & L5 & 27.5 & 2.7 & 11.4 & 5.7 \\
\hline $4 \mathrm{H}$ & $\begin{array}{lc}\text { Mer-Benz E } & 320 \\
\text { 4matic(Wagon) }\end{array}$ & 4 & 4,250 & 221 & L5 & 26 & 2.5 & 10.5 & 0.6 \\
\hline $5 \mathrm{~L}$ & Toyota Avalon & $\mathrm{F}$ & 3,750 & 210 & L4 & 28.1 & 2.6 & 10.2 & 11.4 \\
\hline $6 \mathrm{~L}$ & Saturn Sc & $\mathrm{F}$ & 2,750 & 124 & S5 & 38.2 & 4.3 & 15.5 & 0.6 \\
\hline 7 & $\begin{array}{l}\text { Chevrolet } \mathrm{S} 10 \mathrm{P} / \mathrm{U} \\
2 \mathrm{wd}\end{array}$ & $\mathrm{R}$ & 3,500 & 120 & S5 & 29.4 & 3.6 & 15.8 & 3.3 \\
\hline 7 & GMC Sonoma 2wd & $\mathrm{R}$ & 3,375 & 120 & S5 & 29.4 & 3.2 & 14.1 & 0.7 \\
\hline 7 & Ford Ranger $4 \times 2$ & $\mathrm{R}$ & 3,500 & 119 & M5 & 28.5 & 2.9 & 12.9 & 6.3 \\
\hline 7 & Mazda Mazda 4x2 & $\mathrm{R}$ & 3,500 & 119 & M5 & 28.5 & 2.9 & 12.9 & 1.1 \\
\hline 11 & Subaru Forester & 4 & 3,500 & 165 & M5 & 27.9 & 4.0 & 18.9 & 0.3 \\
\hline 11 & Subaru Forester & 4 & 3,500 & 165 & L4 & 27.8 & 3.9 & 18.4 & 1.0 \\
\hline 11 & Lexus Rx 300 4wd & 4 & 4,250 & 220 & L4 & 23.4 & 2.8 & 13.5 & 2.8 \\
\hline
\end{tabular}




\begin{tabular}{|c|c|c|c|c|c|c|c|c|c|}
\hline Class & Name & Drive & $\begin{array}{l}\text { Test } \\
\text { Wt. } \\
\text { (lbs) }\end{array}$ & $\begin{array}{l}\text { Horse- } \\
\text { power }\end{array}$ & $\begin{array}{l}\text { Trans- } \\
\text { mission } \\
\text { Type }\end{array}$ & $\begin{array}{l}\text { Fuel } \\
\text { Econ. } \\
\text { (MPG) }\end{array}$ & $\begin{array}{l}\text { Actual } \\
\text { MPG - } \\
\text { Predicted } \\
\text { MPG }\end{array}$ & $\begin{array}{c}\% \\
\text { Diff. } \\
\text { from } \\
\text { Class } \\
\text { Avg } \\
\text { MPG }\end{array}$ & $\begin{array}{l}\text { Sales } \\
\%\end{array}$ \\
\hline 11 & $\begin{array}{l}\text { Mitsubishi Montero } \\
\text { Sport/Nativa }\end{array}$ & $\mathrm{R}$ & 4,000 & 132 & M5 & 26.7 & 2.5 & 12.0 & 0.0 \\
\hline 11 & Lexus Rx 300 2wd & $\mathrm{F}$ & 4,000 & 220 & L4 & 24.2 & 2.2 & 10.4 & 1.5 \\
\hline 12 & $\begin{array}{l}\text { Chevrolet } \\
\text { Tahoe 4wd }\end{array}$ & 4 & 5,750 & 255 & E4 & 18.6 & 1.8 & 10.2 & 2.9 \\
\hline 13 & Toyota Rav4 2wd & $\mathrm{F}$ & 3,125 & 127 & L4 & 30.5 & 3.5 & 12.9 & 4.7 \\
\hline 13 & Toyota Rav4 2wd & $\mathrm{F}$ & 3,000 & 127 & M5 & 30.9 & 3.4 & 12.7 & 0.6 \\
\hline 13 & $\begin{array}{l}\text { Toyota Rav4 Soft Top } \\
\text { 2wd }\end{array}$ & $\mathrm{F}$ & 3,000 & 127 & M5 & 30.9 & 3.4 & 12.7 & 0.0 \\
\hline 13 & $\begin{array}{l}\text { Toyota Rav4 Soft Top } \\
2 \text { wd }\end{array}$ & $\mathrm{F}$ & 3,000 & 127 & L4 & 30.5 & 3.0 & 11.2 & 0.0 \\
\hline 13 & $\begin{array}{l}\text { Toyota Rav4 Soft Top } \\
\text { 4wd }\end{array}$ & 4 & 3,250 & 127 & L4 & 28.3 & 2.9 & 10.5 & 0.0 \\
\hline
\end{tabular}

Drive: $\mathrm{R}=$ rear-wheel drive, $\mathrm{F}=$ front-wheel drive, $4=$ four-wheel drive

Transmission types: $\mathrm{M}=$ manual, $\mathrm{S}=$ manual with creeper, $\mathrm{L}=$ lockup automatic, $\mathrm{E}=$ =electric automatic; number in code represents the number of speeds.

\section{FINDINGS}

This ranking system selects a higher percentage of cars with manual transmissions than is observed in the general population. This method also selects almost no luxury class cars, which raises the issue as to whether high performance cars should be eligible for an energy-efficient rating. However, the fact that the MercedesBenz E320 was selected indicates that they are not completely excluded.

\section{AVOIDING CLASSES}

All of the methods discussed thus far use the CEC classification system. However, using a volume-based class method may open the door to potential gaming of the system. One means of shutting this door is to avoid this classification system and to treat volume as a continuous variable, with the other variables accounting for performance.

This ranking system uses the volume-based regressions shown in the table below, which have already been shown to give rankings that are very similar to those of the MPG ranking system. However, rather than ranking them within volume-based classes, the vehicles are simply ranked in descending order of the residual (i.e., the difference between the actual and predicted MPG). A threshold criterion is established where the residuals must exceed predicted fuel economy by at least 5 percent. 
Table A8. Regressions Used

\begin{tabular}{l|rr|rr}
\hline & \multicolumn{2}{|c|}{ Cars } & \multicolumn{2}{c}{ Trucks } \\
& $\begin{array}{l}\text { Regression } \\
\text { Coefficient }\end{array}$ & $\mathrm{T}$ - Statistic & $\begin{array}{l}\text { Regression } \\
\text { Coefficient }\end{array}$ & $\mathrm{T}$ - Statistic \\
\hline Log $(\mathrm{HP} / \mathrm{Wt})$ & -0.58 & -25.21 & -0.36 & -6.3 \\
Log (Volume) & -0.13 & -4.01 & -0.32 & -12.98 \\
4-Wheel Drive & -0.03 & -3.63 & -0.04 & -5.98 \\
Manual Transmission & 0.03 & 6.62 & 0.02 & 2.28 \\
\hline
\end{tabular}

Table A9. Ranking Results Using Just Residuals

\begin{tabular}{|c|c|c|c|c|c|c|c|c|c|}
\hline Class & Division/Name & & Drive & $\begin{array}{l}\text { Engine } \\
\text { Type }\end{array}$ & $\begin{array}{l}\text { Trans. } \\
\text { Type }\end{array}$ & $\begin{array}{l}\text { Horse- } \\
\text { power }\end{array}$ & $\begin{array}{c}\text { Fuel Economy } \\
\text { (MPG) }\end{array}$ & $\begin{array}{c}\text { Actual MPG } \\
\text { - Predicted } \\
\text { MPG }\end{array}$ & $\begin{array}{c}\text { MPG Diff. } \\
\text { Relative to } \\
\text { Predicted } \\
(\%)\end{array}$ \\
\hline $1 \mathrm{H}$ & Mer-Benz Clk 430 & & $\mathrm{R}$ & V8 & L5 & 275 & 24.2 & 21.68 & 11.6 \\
\hline $1 \mathrm{H}$ & $\begin{array}{l}\text { Mer-Benz } \quad \mathrm{Clk} \\
\text { (Cabriolet) }\end{array}$ & 320 & $\mathrm{R}$ & V6 & L5 & 214 & 27.5 & 26.25 & 5.1 \\
\hline $1 \mathrm{H}$ & Mer-Benz Clk 320 & & $\mathrm{R}$ & V6 & L5 & 214 & 27.2 & 24.55 & 12.0 \\
\hline $1 \mathrm{~L}$ & Ford Escort Zx2 & & $\mathrm{F}$ & L4 & M5 & 130 & 33 & 31.51 & 6.0 \\
\hline $1 \mathrm{~L}$ & Ford Escort Zx2 & & $\mathrm{F}$ & L4 & L4 & 130 & 32.8 & 29.21 & 8.9 \\
\hline $1 \mathrm{~L}$ & Chevy Metro & & $\mathrm{F}$ & L4 & A3 & 79 & 37.4 & 34.92 & 7.7 \\
\hline $1 \mathrm{~L}$ & Toyota Echo & & $\mathrm{F}$ & L4 & M5 & 108 & 43.1 & 30.11 & 43.1 \\
\hline $1 \mathrm{~L}$ & Toyota Echo & & $\mathrm{F}$ & L4 & L4 & 108 & 39.5 & 28.79 & 37.2 \\
\hline $2 \mathrm{~L}$ & Honda Civic Ex & & $\mathrm{F}$ & L4 & L4 & 127 & 35.6 & 29.53 & 20.6 \\
\hline $2 \mathrm{~L}$ & Nissan Sentra & & $\mathrm{F}$ & L4 & L4 & 126 & 33.9 & 28.71 & 18.1 \\
\hline $2 \mathrm{~L}$ & Toyota Corolla & & $\mathrm{F}$ & L4 & L3 & 125 & 34.9 & 28.80 & 21.2 \\
\hline $2 \mathrm{~L}$ & Toyota Corolla & & $\mathrm{F}$ & L4 & L4 & 125 & 37.4 & 28.80 & 29.9 \\
\hline $3 \mathrm{H}$ & $\begin{array}{ll}\text { Mer-Benz } & \text { C } \\
\text { Kompressor } & \end{array}$ & 230 & $\mathrm{R}$ & L4 & L5 & 185 & 28.5 & 26.40 & 7.6 \\
\hline $3 \mathrm{H}$ & Subaru Legacy Sw & & 4 & $\mathrm{H} 4$ & L4 & 165 & 27.8 & 26.09 & 6.5 \\
\hline $3 \mathrm{H}$ & Acura Acura 3.2tl & & $\mathrm{F}$ & V6 & L5 & 225 & 26.2 & 24.25 & 9.7 \\
\hline $3 \mathrm{~L}$ & Dodge Neon & & $\mathrm{F}$ & L4 & L3 & 132 & 30.9 & 28.41 & 8.8 \\
\hline $3 \mathrm{~L}$ & Honda Civic Dx 4dr & & $\mathrm{F}$ & L4 & L4 & 106 & 35.8 & 30.74 & 16.4 \\
\hline $4 \mathrm{H}$ & Mer-Benz E 320 & & $\mathrm{R}$ & V6 & L5 & 221 & 27.5 & 25.02 & 10.3 \\
\hline $4 \mathrm{H}$ & Cadillac Seville & & $\mathrm{F}$ & V8 & E4 & 300 & 24.1 & 21.86 & 10.2 \\
\hline $4 \mathrm{H}$ & Infinity Infiniti I30 & & $\mathrm{F}$ & V6 & L4 & 227 & 26.5 & 23.48 & 12.4 \\
\hline $4 \mathrm{~L}$ & Honda Accord Coupe & & $\mathrm{F}$ & V6 & L4 & 200 & 27 & 25.06 & 7.7 \\
\hline $4 \mathrm{~L}$ & Nissan Maxima & & $\mathrm{F}$ & L6 & L4 & 222 & 26.6 & 23.31 & 14.1 \\
\hline
\end{tabular}




\begin{tabular}{|c|c|c|c|c|c|c|c|c|}
\hline Class & Division/Name & Drive & $\begin{array}{l}\text { Engine } \\
\text { Type }\end{array}$ & $\begin{array}{l}\text { Trans. } \\
\text { Type }\end{array}$ & $\begin{array}{l}\text { Horse- } \\
\text { power }\end{array}$ & $\begin{array}{c}\text { Fuel Economy } \\
\text { (MPG) }\end{array}$ & $\begin{array}{l}\text { Actual MPG } \\
\text { - Predicted } \\
\text { MPG }\end{array}$ & $\begin{array}{c}\text { MPG Diff. } \\
\text { Relative to } \\
\text { Predicted } \\
(\%)\end{array}$ \\
\hline $5 \mathrm{H}$ & Cadillac Deville & $\mathrm{F}$ & V8 & E4 & 275 & 24.1 & 22.68 & 5.8 \\
\hline $5 \mathrm{~L}$ & Chrysler $300 \mathrm{M}$ & $\mathrm{F}$ & V6 & L4 & 253 & 24.6 & 22.84 & 5.5 \\
\hline $5 \mathrm{~L}$ & Toyota Avalon & $\mathrm{F}$ & V6 & L4 & 210 & 28.1 & 24.92 & 12.8 \\
\hline $6 \mathrm{H}$ & Chevy Corvette & $\mathrm{R}$ & V8 & M6 & 345 & 24.9 & 21.34 & 17.6 \\
\hline $6 \mathrm{H}$ & Chevy Corvette & $\mathrm{R}$ & V8 & E4 & 345 & 23.2 & 19.78 & 14.8 \\
\hline $6 \mathrm{~L}$ & Mitsubishi Eclipse & $\mathrm{F}$ & V6 & L4 & 205 & 26.7 & 24.72 & 5.6 \\
\hline $6 \mathrm{~L}$ & Toyota Celica & $\mathrm{F}$ & L4 & L4 & 140 & 34.9 & 27.41 & 27.3 \\
\hline 9 & Ford Windstar Wagon & $\mathrm{F}$ & V8 & E4 & 200 & 22.4 & 21.0 & 6.5 \\
\hline 9 & Chevrolet Venture & $\mathrm{F}$ & V6 & E4 & 185 & 25 & 20.9 & 19.5 \\
\hline 9 & Honda Odyssey & $\mathrm{F}$ & V6 & L4 & 210 & 23.9 & 20.4 & 17.2 \\
\hline 9 & Toyota Sienna & $\mathrm{F}$ & V8 & L4 & 194 & 24.1 & 20.9 & 15.6 \\
\hline 10 & Ford E150 Van & $\mathrm{R}$ & L4 & E4 & 215 & 19.4 & 17.4 & 11.3 \\
\hline 10 & $\begin{array}{l}\text { Chevrolet G15/25 Chevy } \\
\text { Van }\end{array}$ & $\mathrm{R}$ & V6 & E4 & 200 & 18.8 & 17.8 & 5.9 \\
\hline 10 & $\begin{array}{l}\text { Chevrolet G15/25 Chevy } \\
\text { Van }\end{array}$ & $\mathrm{R}$ & L4 & E4 & 255 & 18.8 & 16.8 & 11.6 \\
\hline 13 & $\begin{array}{l}\text { Chevrolet Tracker Van } \\
4 \times 4\end{array}$ & 4 & L4 & L4 & 127 & 27.6 & 23.2 & 18.8 \\
\hline 13 & Honda Cr-V & 4 & V6 & L4 & 146 & 27.1 & 21.6 & 25.6 \\
\hline 13 & Honda Cr-V & $\mathrm{R}$ & V6 & L4 & 146 & 27.5 & 23.4 & 17.4 \\
\hline
\end{tabular}

FINDINGS

It is notable that several vehicle classes have been omitted completely using this ranking system. No standard or compact pickups were selected, nor were any standard or compact SUVs (only mini-SUVs). Otherwise, this criterion returns a similar list of vehicles as the method ranking vehicles based on MPG within class.

The results contain only three manual-transmission vehicles, which is in line with the small number of these vehicles typically sold. 



\section{APPENDIX B}

\section{VEHICLES SELECTED BY EACH ENERGY STARY METHOD}





\section{APPENDIX B: VEHICLES SELECTED BY EACH ENERGY STAR METHOD}

Energy Star Method 1 Vehicle List

Top $20 \%$ of vehicles by MPG

\begin{tabular}{|c|c|c|c|c|c|c|c|c|c|c|c|}
\hline $\begin{array}{l}\text { Type-Size } \\
\text { Class }\end{array}$ & Manufacturer/Division & Name & $\begin{array}{l}\text { Dom I } \\
\text { Imp }\end{array}$ & DR & Cyl & Disp & Fuel & Trans & MPG & GHG & $\begin{array}{l}\text { Oil } \\
\text { Use }\end{array}$ \\
\hline \multicolumn{12}{|l|}{ Cars } \\
\hline $\mathrm{C} 4$ & DAEWOO & LANOS- 3Dr & 1 & $\mathrm{~F}$ & L4 & 1.5 & $\mathrm{G}$ & $M$ & 34.8 & 5.5 & 10.3 \\
\hline C4 & DAEWOO & LANOS-3Dr & I & $\mathrm{F}$ & L4 & 1.6 & G & M & 34.6 & 5.5 & 10.3 \\
\hline C4 & DAEWOO & LANOS- 3Dr & I & $\mathrm{F}$ & L4 & 1.5 & G & A & 32.9 & 5.8 & 10.8 \\
\hline C4 & DAEWOO & LANOS-4Dr & I & $\mathrm{F}$ & L4 & 1.6 & $\mathrm{G}$ & M & 31.8 & 6.0 & 11.2 \\
\hline C4 & DAEWOO & LANOS-3Dr & I & $\mathrm{F}$ & L4 & 1.6 & $\mathrm{G}$ & $A$ & 31.2 & 6.1 & 11.4 \\
\hline C4 & DAEWOO & NUBIRA & I & $\mathrm{F}$ & L4 & 2.0 & G & M & 30.1 & 6.3 & 11.8 \\
\hline $\mathrm{C7}$ & DAEWOO & $\begin{array}{l}\text { NUBIRA } \\
\text { WAGON }\end{array}$ & I & $\mathrm{F}$ & L4 & 2.0 & G & M & 30.1 & 6.3 & 11.8 \\
\hline $\mathrm{C} 7$ & DAEWOO & $\begin{array}{l}\text { NUBIRA } \\
\text { WAGON }\end{array}$ & 1 & $\mathrm{~F}$ & L4 & 4.0 & G & M & 30.1 & 6.3 & 11.8 \\
\hline C4 & DAEWOO & LANOS-4Dr & 1 & $\mathrm{~F}$ & L4 & 1.6 & G & $A$ & 29.7 & 6.4 & 12.0 \\
\hline C4 & DAEWOO & NUBIRA & I & $\mathrm{F}$ & L4 & 2.0 & G & $A$ & 29.5 & 6.5 & 12.1 \\
\hline $\mathrm{C7}$ & DAEWOO & $\begin{array}{l}\text { NUBIRA } \\
\text { WAGON }\end{array}$ & I & $\mathrm{F}$ & L4 & 2.0 & G & A & 29.5 & 6.5 & 12.1 \\
\hline $\mathrm{C} 7$ & DAEWOO & $\begin{array}{l}\text { NUBIRA } \\
\text { WAGON }\end{array}$ & 1 & $\mathrm{~F}$ & L4 & 3.0 & G & A & 29.5 & 6.5 & 12.1 \\
\hline C4 & DAEWOO & NUBIRA & I & $\mathrm{F}$ & L4 & 2.0 & G & M & 30.1 & 6.3 & 11.8 \\
\hline C4 & DAEWOO & NUBIRA & 1 & $\mathrm{~F}$ & L4 & 2.0 & G & $A$ & 29.5 & 6.5 & 12.1 \\
\hline C3 & DCC - CHRYSLER & NEON & $\mathrm{D}$ & $\mathrm{F}$ & L4 & 2.0 & G & M & 36.1 & 5.3 & 9.9 \\
\hline C4 & DCC - CHRYSLER & CIRRUS & $\mathrm{D}$ & $\mathrm{F}$ & L4 & 2.0 & G & M & 34.3 & 5.6 & 10.4 \\
\hline C3 & DCC - CHRYSLER & NEON & $\mathrm{D}$ & $\mathrm{F}$ & L4 & 2.0 & G & $A$ & 31.9 & 6.0 & 11.2 \\
\hline C3 & DCC - DODGE & NEON & $\mathrm{D}$ & $\mathrm{F}$ & L4 & 2.0 & $\mathrm{G}$ & $M$ & 36.1 & 5.3 & 9.9 \\
\hline C3 & DCC - DODGE & NEON & $\mathrm{D}$ & $\mathrm{F}$ & L4 & 2.0 & G & $A$ & 31.9 & 6.0 & 11.2 \\
\hline C3 & DCC - PLYMOUTH & NEON & $\mathrm{D}$ & $\mathrm{F}$ & L4 & 2.0 & G & M & 36.1 & 5.3 & 9.9 \\
\hline C4 & DCC - PLYMOUTH & BREEZE & $\mathrm{D}$ & $\mathrm{F}$ & L4 & 2.0 & G & M & 35.4 & 5.4 & 10.1 \\
\hline C3 & DCC - PLYMOUTH & NEON & $\mathrm{D}$ & $\mathrm{F}$ & L4 & 2.0 & $\mathrm{G}$ & $A$ & 31.9 & 6.0 & 11.2 \\
\hline $\mathrm{C7}$ & FMC - FORD & FOCUS WAGON & $\mathrm{D}$ & $\mathrm{F}$ & L4 & 2.0 & G & M & 36.2 & 5.3 & 9.9 \\
\hline C3 & FMC - FORD & FOCUS & $\mathrm{D}$ & $\mathrm{F}$ & L4 & 2.0 & G & $M$ & 35.7 & 5.4 & 10.0 \\
\hline C3 & FMC - FORD & ESCORT & $\mathrm{D}$ & $F$ & L4 & 2.0 & G & M & 35.7 & 5.4 & 10.0 \\
\hline C3 & FMC - FORD & FOCUS & $\mathrm{D}$ & $\mathrm{F}$ & L4 & 2.0 & G & $A$ & 33.9 & 5.7 & 10.5 \\
\hline $\mathrm{C7}$ & FMC - FORD & FOCUS WAGON & $\mathrm{D}$ & $\mathrm{F}$ & L4 & 2.0 & $\mathrm{G}$ & $A$ & 32.6 & 5.9 & 11.0 \\
\hline C3 & FMC - FORD & ESCORT & $\mathrm{D}$ & $\mathrm{F}$ & L4 & 2.0 & $\mathrm{G}$ & $A$ & 33.7 & 5.7 & 10.6 \\
\hline C2 & FMC - FORD & ESCORT ZX2 & $\mathrm{D}$ & $\mathrm{F}$ & L4 & 2.0 & G & M & 33.0 & 5.8 & 10.8 \\
\hline C3 & FMC - FORD & FOCUS 3D & $\mathrm{D}$ & $\mathrm{F}$ & L4 & 2.0 & G & M & 33.0 & 5.8 & 10.8 \\
\hline C3 & FMC - FORD & FOCUS 3D & $\mathrm{D}$ & $F$ & L4 & 2.0 & G & $A$ & 32.4 & 5.9 & 11.0 \\
\hline $\mathrm{C} 2$ & FMC - FORD & ESCORT ZX2 & $\mathrm{D}$ & $\mathrm{F}$ & L4 & 2.0 & G & $A$ & 33.1 & 5.8 & 10.8 \\
\hline $\mathrm{C7}$ & FMC - FORD & FOCUS WAGON & $\mathrm{D}$ & $\mathrm{F}$ & L4 & 2.0 & G & $A$ & 32.6 & 5.9 & 11.0 \\
\hline C3 & FMC - FORD & CONTOUR & $\mathrm{D}$ & $\mathrm{F}$ & L4 & 2.0 & G & $M$ & 32.6 & 5.9 & 11.0 \\
\hline C3 & FMC - FORD & CONTOUR & $\mathrm{D}$ & $\mathrm{F}$ & L4 & 2.0 & G & $A$ & 30.3 & 6.3 & 11.8 \\
\hline C3 & FMC - FORD & CONTOUR & $\mathrm{D}$ & $\mathrm{F}$ & L4 & 2.0 & $\mathrm{G}$ & $A$ & 30.3 & 6.3 & 11.8 \\
\hline C3 & FMC - MAZDA & PROTEGE & 1 & $\mathrm{~F}$ & L4 & 1.8 & $G$ & $A$ & 30.3 & 6.3 & 11.8 \\
\hline
\end{tabular}




\begin{tabular}{|c|c|c|c|c|c|c|c|c|c|c|c|}
\hline $\begin{array}{l}\text { Type-Size } \\
\text { Class }\end{array}$ & Manufacturer/Division & Name & $\begin{array}{l}\text { Dom I } \\
\text { Imp }\end{array}$ & DR & Cyl & Disp & Fuel & Trans & MPG & GHG & $\begin{array}{l}\text { Oil } \\
\text { Use }\end{array}$ \\
\hline $\mathrm{C} 3$ & FMC - MAZDA & PROTEGE & $\mathrm{I}$ & $\bar{F}$ & $\mathrm{~L} 4$ & 1.6 & $\mathrm{G}$ & $\bar{A}$ & 33.8 & 5.7 & 10.6 \\
\hline C4 & FMC - MAZDA & 626 & I & $\mathrm{F}$ & L4 & 2.0 & G & M & 33.5 & 5.7 & 10.6 \\
\hline C3 & FMC - MAZDA & PROTEGE & $\mathrm{I}$ & $\mathrm{F}$ & $\mathrm{L} 4$ & 1.8 & G & $M$ & 32.4 & 5.9 & 11.0 \\
\hline C6 & FMC - MAZDA & MX-5 MIATA & I & $\mathrm{R}$ & L4 & 1.8 & G & M & 31.4 & 6.1 & 11.4 \\
\hline C3 & FMC - MAZDA & PROTEGE & I & $\mathrm{F}$ & L4 & 1.6 & $\mathrm{G}$ & M & 36.5 & 5.3 & 9.8 \\
\hline C6 & FMC - MAZDA & MX-5 MIATA & I & $\mathrm{R}$ & L4 & 1.8 & G & M & 30.2 & 6.3 & 11.8 \\
\hline C3 & FMC - MERCURY & MYSTIQUE & D & $\mathrm{F}$ & L4 & 2.0 & G & M & 32.4 & 5.9 & 11.0 \\
\hline C3 & FMC - MERCURY & COUGAR & D & $\mathrm{F}$ & L4 & 2.0 & G & M & 32.4 & 5.9 & 11.0 \\
\hline C3 & FMC - MERCURY & MYSTIQUE & D & $\mathrm{F}$ & L4 & 2.0 & G & A & 29.6 & 6.5 & 12.1 \\
\hline $\mathrm{C} 7$ & FUJI - SUBARU & IMPREZA SW & I & 4 & $\mathrm{H} 4$ & 2.2 & G & A & 29.4 & 6.5 & 12.1 \\
\hline $\mathrm{C} 2$ & GMC - CHEVY & METRO & 1 & $\mathrm{~F}$ & L3 & 1.0 & G & M & 48.9 & 4.0 & 7.3 \\
\hline $\mathrm{C} 2$ & GMC - CHEVY & METRO & 1 & $\mathrm{~F}$ & L4 & 1.3 & G & M & 45.5 & 4.2 & 7.8 \\
\hline C3 & GMC - CHEVY & PRIZM & I & $\mathrm{F}$ & L4 & 1.8 & G & M & 39.6 & 4.9 & 9.0 \\
\hline C3 & GMC - CHEVY & PRIZM & I & $\mathrm{F}$ & L4 & 1.8 & G & A & 37.5 & 5.1 & 9.5 \\
\hline C2 & GMC - CHEVY & METRO & I & $\mathrm{F}$ & L4 & 1.3 & $\mathrm{G}$ & $A$ & 37.6 & 5.1 & 9.5 \\
\hline C3 & GMC - CHEVY & PRIZM & I & $\mathrm{F}$ & L4 & 1.8 & G & A & 34.9 & 5.5 & 10.2 \\
\hline $\mathrm{C} 2$ & GMC - CHEVY & CAVALIER & D & $\mathrm{F}$ & L4 & 2.2 & G & M & 32.1 & 6.0 & 11.1 \\
\hline C2 & GMC - CHEVY & CAVALIER & $\mathrm{D}$ & $\mathrm{F}$ & L4 & 2.2 & G & A & 30.7 & 6.2 & 11.6 \\
\hline C2 & GMC - CHEVY & CAVALIER Z24 & D & $\mathrm{F}$ & L4 & 2.4 & G & M & 30.8 & 6.2 & 11.6 \\
\hline $\mathrm{C} 2$ & GMC - CHEVY & CAVALIER Z24 & D & $\mathrm{F}$ & L4 & 2.4 & G & A & 29.4 & 6.5 & 12.1 \\
\hline C3 & GMC - OLDSMOBILE & ALERO & D & $\mathrm{F}$ & L4 & 2.4 & G & M & 29.5 & 6.5 & 12.1 \\
\hline C3 & GMC - OLDSMOBILE & ALERO & D & $\mathrm{F}$ & L4 & 2.4 & G & A & 29.4 & 6.5 & 12.1 \\
\hline $\mathrm{C} 2$ & GMC - PONTIAC & SUNFIRE & D & $\mathrm{F}$ & L4 & 2.2 & G & M & 32.1 & 6.0 & 11.1 \\
\hline $\mathrm{C} 2$ & GMC - PONTIAC & SUNFIRE CNVT & D & $\mathrm{F}$ & L4 & 2.2 & G & A & 30.7 & 6.2 & 11.6 \\
\hline $\mathrm{C} 2$ & GMC - PONTIAC & SUNFIRE & $\mathrm{D}$ & $\mathrm{F}$ & L4 & 2.4 & G & M & 30.8 & 6.2 & 11.6 \\
\hline C3 & GMC - PONTIAC & GRAND AM & D & $\mathrm{F}$ & L4 & 2.4 & G & M & 29.5 & 6.5 & 12.1 \\
\hline C2 & GMC - PONTIAC & SUNFIRE & D & $\mathrm{F}$ & L4 & 2.4 & G & A & 29.4 & 6.5 & 12.1 \\
\hline C3 & GMC - PONTIAC & GRAND AM & D & $\mathrm{F}$ & L4 & 2.4 & G & A & 29.4 & 6.5 & 12.1 \\
\hline C3 & GMC - SATURN & SL & D & $\mathrm{F}$ & L4 & 1.9 & G & M & 38.7 & 5.0 & 9.2 \\
\hline $\mathrm{C} 2$ & GMC - SATURN & SC & D & $\mathrm{F}$ & L4 & 1.9 & G & M & 35.4 & 5.4 & 10.1 \\
\hline $\mathrm{C} 2$ & GMC - SATURN & SC & D & $\mathrm{F}$ & L4 & 1.9 & G & M & 38.1 & 5.0 & 9.4 \\
\hline C3 & GMC - SATURN & SL & D & $\mathrm{F}$ & L4 & 1.9 & G & M & 36.1 & 5.3 & 9.9 \\
\hline $\mathrm{C} 7$ & GMC - SATURN & SW & D & $\mathrm{F}$ & L4 & 1.9 & G & M & 36.1 & 5.3 & 9.9 \\
\hline C3 & GMC - SATURN & SL & D & $\mathrm{F}$ & L4 & 1.9 & G & A & 34.4 & 5.6 & 10.4 \\
\hline $\mathrm{C} 2$ & GMC - SATURN & SC & D & $\mathrm{F}$ & L4 & 1.9 & G & A & 33.9 & 5.7 & 10.5 \\
\hline $\mathrm{C} 7$ & GMC - SATURN & SW & D & $\mathrm{F}$ & L4 & 1.9 & G & A & 35.4 & 5.4 & 10.1 \\
\hline C2 & GMC - SATURN & SC & D & $\mathrm{F}$ & L4 & 1.9 & G & A & 36.1 & 5.3 & 9.9 \\
\hline C3 & GMC - SATURN & SL & D & $\mathrm{F}$ & L4 & 1.9 & G & A & 36.0 & 5.3 & 9.9 \\
\hline $\mathrm{C} 7$ & GMC - SATURN & SW & D & $\mathrm{F}$ & L4 & 1.9 & G & A & 33.9 & 5.7 & 10.5 \\
\hline C4 & GMC - SATURN & LS & D & $\mathrm{F}$ & L4 & 2.2 & G & M & 31.9 & 6.0 & 11.2 \\
\hline C4 & GMC - SATURN & LS & D & $\mathrm{F}$ & L4 & 2.2 & G & A & 30.5 & 6.3 & 11.7 \\
\hline C8 & GMC - SATURN & LW & D & $\mathrm{F}$ & $\mathrm{L} 4$ & 2.2 & G & A & 29.4 & 6.5 & 12.1 \\
\hline C2 & HONDA - ACURA & $\begin{array}{l}\text { ACURA } \\
\text { INTEGRA }\end{array}$ & I & $\mathrm{F}$ & L4 & 1.8 & G & M & 32.3 & 5.9 & 11.0 \\
\hline C2 & HONDA - ACURA & $\begin{array}{l}\text { ACURA } \\
\text { INTEGRA -R }\end{array}$ & 1 & $\mathrm{~F}$ & L4 & 1.8 & G & M & 31.9 & 6.0 & 11.2 \\
\hline $\mathrm{C} 2$ & HONDA - ACURA & $\begin{array}{l}\text { ACURA } \\
\text { INTEGRA }\end{array}$ & I & $\mathrm{F}$ & L4 & 1.8 & G & A & 30.7 & 6.2 & 11.6 \\
\hline C6 & HONDA & INSIGHT & I & $\mathrm{F}$ & L3 & 1.0 & G/EL & M & 76.3 & 2.6 & 4.7 \\
\hline
\end{tabular}




\begin{tabular}{|c|c|c|c|c|c|c|c|c|c|c|c|}
\hline $\begin{array}{l}\text { Type-Size } \\
\text { Class }\end{array}$ & Manufacturer/Division & Name & $\begin{array}{l}\text { Dom I } \\
\text { Imp }\end{array}$ & DR & Cyl & Disp & Fuel & Trans & MPG & GHG & $\begin{array}{l}\text { Oil } \\
\text { Use }\end{array}$ \\
\hline $\mathrm{C} 2$ & HONDA & CIVIC HX & 1 & $\bar{F}$ & $\mathrm{~L} 4$ & 1.6 & $\bar{G}$ & $M$ & 44.7 & 4.3 & 8.0 \\
\hline $\mathrm{C} 2$ & HONDA & CIVIC HX & I & $\mathrm{F}$ & L4 & 1.6 & G & A & 41.9 & 4.6 & 8.5 \\
\hline $\mathrm{C} 2$ & HONDA & CIVIC dx 4Dr & I & $F$ & L4 & 1.6 & $\mathrm{G}$ & $M$ & 37.2 & 5.2 & 9.6 \\
\hline $\mathrm{C} 2$ & HONDA & CIVIC ex & I & $\mathrm{F}$ & L4 & 1.6 & G & M & 37.2 & 5.2 & 9.6 \\
\hline $\mathrm{C} 2$ & HONDA & CIVIC vp & I & $\mathrm{F}$ & L4 & 1.6 & G & A & 35.5 & 5.4 & 10.1 \\
\hline $\mathrm{C} 2$ & HONDA & CIVIC dx 4Dr & I & $\mathrm{F}$ & L4 & 1.6 & G & A & 35.6 & 5.4 & 10.0 \\
\hline $\mathrm{C} 2$ & HONDA & CIVIC ex & I & $\mathrm{F}$ & L4 & 1.6 & G & A & 35.5 & 5.4 & 10.1 \\
\hline $\mathrm{C} 2$ & HONDA & CIVIC si & 1 & $\mathrm{~F}$ & L4 & 1.6 & G & M & 32.8 & 5.8 & 10.9 \\
\hline C4 & HONDA & ACCORD Ix & I & $\mathrm{F}$ & L4 & 2.3 & G & M & 32.1 & 6.0 & 11.1 \\
\hline C4 & HONDA & ACCORD & I & $\mathrm{F}$ & L4 & 2.3 & G & M & 30.4 & 6.3 & 11.8 \\
\hline $\mathrm{C} 4$ & HONDA & ACCORD IX & I & $\mathrm{F}$ & L4 & 2.3 & G & A & 29.9 & 6.4 & 11.9 \\
\hline $\mathrm{C} 3$ & HYUNDAI & ACCENT & I & $\mathrm{F}$ & L4 & 1.5 & G & M & 36.6 & 5.3 & 9.8 \\
\hline $\mathrm{C} 3$ & HYUNDAI & ACCENT & I & $\mathrm{F}$ & L4 & 1.5 & G & A & 34.2 & 5.6 & 10.4 \\
\hline $\mathrm{C} 3$ & HYUNDAI & ELANTRA & I & $\mathrm{F}$ & L4 & 2.0 & G & M & 32.1 & 6.0 & 11.1 \\
\hline $\mathrm{C7}$ & HYUNDAI & $\begin{array}{l}\text { ELANTRA } \\
\text { WAGON }\end{array}$ & I & $\mathrm{F}$ & L4 & 2.0 & G & M & 31.3 & 6.1 & 11.4 \\
\hline $\mathrm{C} 2$ & HYUNDAI & TIBURON & I & $\mathrm{F}$ & L4 & 2.0 & G & M & 31.1 & 6.1 & 11.5 \\
\hline $\mathrm{C} 3$ & $\mathrm{KIA}$ & SEPHIA L4 & 1 & $\mathrm{~F}$ & L4 & 1.8 & G & $A$ & 29.9 & 6.4 & 12.0 \\
\hline $\mathrm{C} 3$ & $\mathrm{KIA}$ & SEPHIA M5 & I & $\mathrm{F}$ & L4 & 1.8 & G & M & 29.5 & 6.5 & 12.1 \\
\hline $\mathrm{C} 2$ & MITSUBISHI & MIRAGE 2D & I & $\mathrm{F}$ & L4 & 1.5 & G & M & 41.7 & 4.6 & 8.6 \\
\hline $\mathrm{C} 2$ & MITSUBISHI & MIRAGE & 1 & $\mathrm{~F}$ & L4 & 1.8 & G & M & 36.3 & 5.3 & 9.8 \\
\hline $\mathrm{C} 2$ & MITSUBISHI & MIRAGE 2D & I & $\mathrm{F}$ & L4 & 1.5 & G & $A$ & 36.7 & 5.2 & 9.7 \\
\hline $\mathrm{C} 2$ & MITSUBISHI & MIRAGE & I & $\mathrm{F}$ & L4 & 1.8 & G & $A$ & 33.2 & 5.8 & 10.8 \\
\hline $\mathrm{C} 2$ & MITSUBISHI & ECLIPSE & 1 & $\mathrm{~F}$ & L4 & 2.4 & G & M & 30.1 & 6.3 & 11.9 \\
\hline $\mathrm{C} 2$ & MITSUBISHI & ECLIPSE & 1 & $F$ & L4 & 2.4 & G & A & 30.1 & 6.3 & 11.9 \\
\hline $\mathrm{C} 3$ & NISSAN - INFINITY & INFINITI G20 & 1 & $\mathrm{~F}$ & L4 & 2.0 & G & M & 31.0 & 6.2 & 11.5 \\
\hline $\mathrm{C} 3$ & NISSAN - INFINITY & INFINITI G20 & I & $\mathrm{F}$ & L4 & 2.0 & $\mathrm{G}$ & $A$ & 30.2 & 6.3 & 11.8 \\
\hline $\mathrm{C} 3$ & NISSAN & SENTRA & 1 & $\mathrm{~F}$ & L4 & 1.8 & G & M & 34.9 & 5.5 & 10.2 \\
\hline $\mathrm{C} 3$ & NISSAN & SENTRA & I & $\mathrm{F}$ & L4 & 1.8 & G & A & 33.9 & 5.6 & 10.5 \\
\hline $\mathrm{C} 3$ & NISSAN & SENTRA & I & $\mathrm{F}$ & L4 & 2.0 & G & M & 30.9 & 6.2 & 11.6 \\
\hline $\mathrm{C} 3$ & NISSAN & ALTIMA & I & $\mathrm{F}$ & L4 & 2.4 & G & M & 30.9 & 6.2 & 11.6 \\
\hline $\mathrm{C} 3$ & NISSAN & SENTRA & 1 & $\mathrm{~F}$ & L4 & 2.0 & $\mathrm{G}$ & $A$ & 30.8 & 6.2 & 11.6 \\
\hline $\mathrm{C} 2$ & SUZUKI & SWIFT & I & $\mathrm{F}$ & L4 & 1.3 & G & M & 45.5 & 4.2 & 7.8 \\
\hline $\mathrm{C} 2$ & SUZUKI & ESTEEM & 1 & $\mathrm{~F}$ & L4 & 1.6 & G & M & 38.6 & 5.0 & 9.3 \\
\hline $\mathrm{C} 2$ & SUZUKI & SWIFT & I & $F$ & L4 & 1.3 & G & A & 37.6 & 5.1 & 9.5 \\
\hline $\mathrm{C} 2$ & SUZUKI & ESTEEM & 1 & $\mathrm{~F}$ & L4 & 1.8 & G & M & 36.2 & 5.3 & 9.9 \\
\hline $\mathrm{C} 7$ & SUZUKI & $\begin{array}{l}\text { ESTEEM } \\
\text { WAGON }\end{array}$ & I & $\mathrm{F}$ & L4 & 1.8 & G & M & 35.1 & 5.5 & 10.2 \\
\hline $\mathrm{C} 2$ & SUZUKI & ESTEEM & I & $\mathrm{F}$ & L4 & 1.6 & G & A & 35.0 & 5.5 & 10.2 \\
\hline $\mathrm{C7}$ & SUZUKI & $\begin{array}{l}\text { ESTEEM } \\
\text { WAGON }\end{array}$ & 1 & $\mathrm{~F}$ & L4 & 1.6 & $G$ & $A$ & 33.7 & 5.7 & 10.6 \\
\hline $\mathrm{C7}$ & SUZUKI & $\begin{array}{l}\text { ESTEEM } \\
\text { WAGON }\end{array}$ & I & $\mathrm{F}$ & L4 & 1.8 & G & $A$ & 33.7 & 5.7 & 10.6 \\
\hline $\mathrm{C} 2$ & SUZUKI & ESTEEM & 1 & $\mathrm{~F}$ & L4 & 1.8 & G & A & 33.7 & 5.7 & 10.6 \\
\hline $\mathrm{C} 4$ & TOYOTA & CAMRY CNG & I & $\mathrm{F}$ & L4 & 2.2 & NG & A & 30.2 & 5.4 & 1.8 \\
\hline C3 & TOYOTA & $\mathrm{ECHO}$ & I & $\mathrm{F}$ & L4 & 1.5 & $G$ & M & 43.1 & 4.5 & 8.3 \\
\hline C3 & TOYOTA & COROLLA & I & $\mathrm{F}$ & L4 & 1.8 & G & M & 39.6 & 4.9 & 9.0 \\
\hline $\mathrm{C} 3$ & TOYOTA & $\mathrm{ECHO}$ & I & $\mathrm{F}$ & L4 & 1.5 & $\mathrm{G}$ & $A$ & 39.4 & 4.9 & 9.1 \\
\hline C3 & TOYOTA & COROLLA & I & $\mathrm{F}$ & L4 & 1.8 & $G$ & $A$ & 37.4 & 5.1 & 9.6 \\
\hline
\end{tabular}




\begin{tabular}{|c|c|c|c|c|c|c|c|c|c|c|c|}
\hline $\begin{array}{l}\text { Type-Size } \\
\text { Class } \\
\end{array}$ & Manufacturer/Division & Name & $\begin{array}{l}\text { Dom I } \\
\text { Imp }\end{array}$ & DR & Cyl & Disp & Fuel & Trans & MPG & GHG & $\begin{array}{l}\text { Oil } \\
\text { Use }\end{array}$ \\
\hline $\mathrm{C} 2$ & TOYOTA & CELICA & $\mathrm{I}$ & $\mathrm{F}$ & $\mathrm{L} 4$ & 1.8 & $\mathrm{G}$ & $\mathrm{M}$ & 35.2 & 5.4 & 10.1 \\
\hline C2 & TOYOTA & CELICA & I & $\mathrm{F}$ & L4 & 1.8 & G & $A$ & 34.9 & 5.5 & 10.2 \\
\hline C3 & TOYOTA & COROLLA & I & $\mathrm{F}$ & L4 & 1.8 & G & A & 34.9 & 5.5 & 10.2 \\
\hline $\mathrm{C} 6$ & TOYOTA & MR2 & 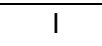 & $\mathrm{F}$ & $\mathrm{L} 4$ & 1.8 & $\mathrm{G}$ & $M$ & 31.8 & 6.0 & 11.2 \\
\hline C3 & TOYOTA & CAMRY SOLARA & I & $\mathrm{F}$ & L4 & 2.2 & G & M & 31.0 & 6.2 & 11.5 \\
\hline C4 & TOYOTA & CAMRY & I & $\mathrm{F}$ & L4 & 2.2 & G & M & 31.0 & 6.2 & 11.5 \\
\hline C2 & TOYOTA & CELICA & I & $\mathrm{F}$ & L4 & 1.8 & G & M & 30.8 & 6.2 & 11.6 \\
\hline C2 & TOYOTA & CELICA & I & $\mathrm{F}$ & L4 & 1.8 & G & A & 30.1 & 6.4 & 11.9 \\
\hline C3 & TOYOTA & CAMRY SOLARA & I & $\mathrm{F}$ & L4 & 2.2 & G & A & 30.1 & 6.4 & 11.9 \\
\hline C4 & TOYOTA & CAMRY & I & $\mathrm{F}$ & L4 & 2.2 & G & A & 30.1 & 6.4 & 11.9 \\
\hline $\mathrm{C} 2$ & TOYOTA & $\begin{array}{l}\text { CAMRY SOLARA } \\
\text { CONVERT }\end{array}$ & I & $\mathrm{F}$ & L4 & 2.2 & G & A & 30.1 & 6.4 & 11.9 \\
\hline C3 & VWA - AUDI & AUDI A4 & I & $\mathrm{F}$ & L4 & 1.8 & G & M & 31.9 & 6.0 & 11.2 \\
\hline C1 & VWA - AUDI & AUDI TT COUPE & I & 4 & L4 & 1.8 & G & M & 29.4 & 6.5 & 12.1 \\
\hline C2 & VWA & NEW BEETLE & I & $\mathrm{F}$ & L4 & 1.9 & $D$ & M & 52.7 & 4.0 & 6.8 \\
\hline C3 & VWA & GOLF TDI & I & $\mathrm{F}$ & L4 & 1.9 & $\mathrm{D}$ & M & 52.7 & 4.0 & 6.8 \\
\hline C3 & VWA & JETTA & I & $\mathrm{F}$ & L4 & 1.9 & $D$ & M & 52.7 & 4.0 & 6.8 \\
\hline C2 & VWA & NEW BEETLE & I & $\mathrm{F}$ & L4 & 1.9 & $D$ & A & 44.7 & 4.7 & 8.0 \\
\hline C3 & VWA & GOLF TDI & I & $\mathrm{F}$ & L4 & 1.9 & $\mathrm{D}$ & A & 44.7 & 4.7 & 8.0 \\
\hline C3 & VWA & JETTA & I & $\mathrm{F}$ & L4 & 1.9 & $D$ & A & 44.7 & 4.7 & 8.0 \\
\hline C2 & VWA & NEW BEETLE & I & $\mathrm{F}$ & L4 & 1.8 & G & M & 32.3 & 5.9 & 11.1 \\
\hline C2 & VWA & CABRIO & I & $\mathrm{F}$ & L4 & 2.0 & G & M & 31.2 & 6.1 & 11.5 \\
\hline C2 & VWA & NEW BEETLE & I & $\mathrm{F}$ & L4 & 2.0 & G & M & 31.2 & 6.1 & 11.5 \\
\hline C3 & VWA & GTI & I & $\mathrm{F}$ & L4 & 2.0 & G & M & 31.2 & 6.1 & 11.5 \\
\hline C3 & VWA & GTI & I & $\mathrm{F}$ & L4 & 1.8 & G & M & 31.3 & 6.1 & 11.4 \\
\hline C3 & VWA & GOLF & 1 & $\mathrm{~F}$ & L4 & 1.8 & G & M & 31.3 & 6.1 & 11.4 \\
\hline C3 & VWA & JETTA & 1 & $\mathrm{~F}$ & L4 & 1.8 & G & M & 31.3 & 6.1 & 11.4 \\
\hline C3 & VWA & GOLF & I & $\mathrm{F}$ & L4 & 2.0 & G & M & 31.2 & 6.1 & 11.5 \\
\hline C3 & VWA & JETTA & I & $\mathrm{F}$ & L4 & 2.0 & G & M & 31.2 & 6.1 & 11.5 \\
\hline C4 & VWA & PASSAT & 1 & $\mathrm{~F}$ & L4 & 1.8 & G & M & 31.3 & 6.1 & 11.4 \\
\hline C8 & VWA & $\begin{array}{l}\text { PASSAT } \\
\text { WAGON }\end{array}$ & 1 & $\mathrm{~F}$ & L4 & 1.8 & G & M & 31.3 & 6.1 & 11.4 \\
\hline \multicolumn{12}{|l|}{ Trucks } \\
\hline$\overline{\mathrm{T} 1}$ & FMC - FORD & $\begin{array}{l}\text { RANGER 4X2 } \\
\text { EV }\end{array}$ & $\mathrm{D}$ & $\mathrm{R}$ & E0 & 2.5 & $\overline{E L}$ & $A$ & 29.7 & 5.2 & 1.8 \\
\hline T7 & GMC - CHEVROLET & $\begin{array}{l}\text { TRACKER } \\
\text { CONV 2WD }\end{array}$ & D & $\mathrm{R}$ & L4 & 1.6 & G & M & 31.1 & 6.2 & 11.5 \\
\hline T7 & GMC - CHEVROLET & $\begin{array}{l}\text { TRACKER } \\
\text { CONV 4X4 }\end{array}$ & D & 4 & L4 & 1.6 & G & M & 30.7 & 6.2 & 11.6 \\
\hline T1 & GMC - CHEVROLET & S10 P/U 2WD & D & $\mathrm{R}$ & L4 & 2.2 & G & M & 29.6 & 6.5 & 12.1 \\
\hline T1 & GMC & SONOMA 2WD & 1 & $\mathrm{R}$ & L4 & 2.2 & G & M & 29.6 & 6.5 & 12.1 \\
\hline $\mathrm{T} 7$ & SUZUKI & $\begin{array}{l}\text { VITARA CONV } \\
2 W D\end{array}$ & 1 & $\mathrm{R}$ & L4 & 1.6 & G & M & 31.1 & 6.1 & 11.5 \\
\hline T7 & SUZUKI & $\begin{array}{l}\text { VITARA CONV } \\
4 \mathrm{WD}\end{array}$ & 1 & 4 & $\mathrm{~L} 4$ & 1.6 & G & M & 30.7 & 6.2 & 11.6 \\
\hline T7 & TOYOTA & RAV4 2WD & I & $\mathrm{F}$ & L4 & 2.0 & G & M & 30.9 & 6.2 & 11.6 \\
\hline T7 & TOYOTA & RAV4 2WD & I & $F$ & L4 & 2.0 & G & A & 30.5 & 6.3 & 11.7 \\
\hline
\end{tabular}


Energy Star Method 2 Vehicle List

Top $20 \%$ of vehicles by Greenhouse Gas Emissions

\begin{tabular}{|c|c|c|c|c|c|c|c|c|c|c|c|}
\hline $\begin{array}{l}\text { Type-Size } \\
\text { Class }\end{array}$ & Manufacturer/Division & Name & $\begin{array}{l}\text { Dom I } \\
\text { Imp }\end{array}$ & DR & Cyl & Disp & Fuel & Trans & MPG & GHG & $\begin{array}{l}\text { Oil } \\
\text { Use }\end{array}$ \\
\hline \multicolumn{12}{|l|}{ Cars } \\
\hline C4 & DAEWOO & LANOS- 3Dr & I & $\mathrm{F}$ & L4 & 1.5 & G & $\mathrm{M}$ & 34.8 & 5.5 & 10.3 \\
\hline C4 & DAEWOO & LANOS-3Dr & I & $\mathrm{F}$ & L4 & 1.6 & G & M & 34.6 & 5.5 & 10.3 \\
\hline C4 & DAEWOO & LANOS- 3Dr & 1 & $\mathrm{~F}$ & L4 & 1.5 & G & $A$ & 32.9 & 5.8 & 10.8 \\
\hline C4 & DAEWOO & LANOS-4Dr & I & $\mathrm{F}$ & L4 & 1.6 & G & M & 31.8 & 6.0 & 11.2 \\
\hline C4 & DAEWOO & LANOS-3Dr & 1 & $\mathrm{~F}$ & L4 & 1.6 & G & $A$ & 31.2 & 6.1 & 11.4 \\
\hline C4 & DAEWOO & NUBIRA & I & $\mathrm{F}$ & L4 & 2.0 & G & M & 30.1 & 6.3 & 11.8 \\
\hline $\mathrm{C7}$ & DAEWOO & NUBIRA WAGON & I & $\mathrm{F}$ & L4 & 2.0 & G & M & 30.1 & 6.3 & 11.8 \\
\hline $\mathrm{C7}$ & DAEWOO & NUBIRA WAGON & I & $\mathrm{F}$ & L4 & 4.0 & G & M & 30.1 & 6.3 & 11.8 \\
\hline C4 & DAEWOO & LANOS-4Dr & I & $\mathrm{F}$ & L4 & 1.6 & G & $A$ & 29.7 & 6.4 & 12.0 \\
\hline C4 & DAEWOO & NUBIRA & I & $\mathrm{F}$ & L4 & 2.0 & G & A & 29.5 & 6.5 & 12.1 \\
\hline $\mathrm{C} 7$ & DAEWOO & NUBIRA WAGON & I & $\mathrm{F}$ & L4 & 2.0 & G & A & 29.5 & 6.5 & 12.1 \\
\hline $\mathrm{C7}$ & DAEWOO & NUBIRA WAGON & I & $\mathrm{F}$ & L4 & 3.0 & G & A & 29.5 & 6.5 & 12.1 \\
\hline C4 & DAEWOO & NUBIRA & I & $\mathrm{F}$ & L4 & 2.0 & G & M & 30.1 & 6.3 & 11.8 \\
\hline C4 & DAEWOO & NUBIRA & I & $\mathrm{F}$ & L4 & 2.0 & G & $A$ & 29.5 & 6.5 & 12.1 \\
\hline $\mathrm{C} 3$ & DCC - CHRYSLER & NEON & D & $\mathrm{F}$ & L4 & 2.0 & G & M & 36.1 & 5.3 & 9.9 \\
\hline $\mathrm{C} 4$ & DCC - CHRYSLER & CIRRUS & D & $\mathrm{F}$ & L4 & 2.0 & G & M & 34.3 & 5.6 & 10.4 \\
\hline $\mathrm{C} 3$ & DCC - CHRYSLER & NEON & D & $\mathrm{F}$ & L4 & 2.0 & G & $A$ & 31.9 & 6.0 & 11.2 \\
\hline $\mathrm{C} 3$ & DCC - DODGE & NEON & D & $\mathrm{F}$ & L4 & 2.0 & G & M & 36.1 & 5.3 & 9.9 \\
\hline $\mathrm{C} 3$ & DCC - DODGE & NEON & D & $\mathrm{F}$ & L4 & 2.0 & G & A & 31.9 & 6.0 & 11.2 \\
\hline $\mathrm{C} 3$ & DCC - PLYMOUTH & NEON & D & $\mathrm{F}$ & L4 & 2.0 & G & M & 36.1 & 5.3 & 9.9 \\
\hline $\mathrm{C} 4$ & DCC - PLYMOUTH & BREEZE & D & $\mathrm{F}$ & L4 & 2.0 & G & M & 35.4 & 5.4 & 10.1 \\
\hline C3 & DCC - PLYMOUTH & NEON & D & $\mathrm{F}$ & L4 & 2.0 & G & A & 31.9 & 6.0 & 11.2 \\
\hline C5 & FMC - FORD & TAURUS FFV & D & $\mathrm{F}$ & V6 & 3.0 & FF & A & 24.6 & 5.9 & 7.8 \\
\hline C8 & FMC - FORD & $\begin{array}{l}\text { TAURUS WAGON } \\
\text { FFV }\end{array}$ & D & $\mathrm{F}$ & V6 & 3.0 & FF & A & 23.3 & 6.2 & 8.3 \\
\hline $\mathrm{C7}$ & FMC - FORD & FOCUS WAGON & $\mathrm{D}$ & $\mathrm{F}$ & L4 & 2.0 & G & M & 36.2 & 5.3 & 9.9 \\
\hline $\mathrm{C} 3$ & FMC - FORD & FOCUS & D & $\mathrm{F}$ & L4 & 2.0 & G & M & 35.7 & 5.4 & 10.0 \\
\hline C3 & FMC - FORD & ESCORT & D & $\mathrm{F}$ & L4 & 2.0 & G & M & 35.7 & 5.4 & 10.0 \\
\hline C3 & FMC - FORD & FOCUS & D & $\mathrm{F}$ & L4 & 2.0 & G & A & 33.9 & 5.7 & 10.5 \\
\hline $\mathrm{C7}$ & FMC - FORD & FOCUS WAGON & $\mathrm{D}$ & $\mathrm{F}$ & L4 & 2.0 & G & A & 32.6 & 5.9 & 11.0 \\
\hline C3 & FMC - FORD & ESCORT & D & $\mathrm{F}$ & L4 & 2.0 & G & A & 33.7 & 5.7 & 10.6 \\
\hline $\mathrm{C} 2$ & FMC - FORD & ESCORT ZX2 & D & $\mathrm{F}$ & L4 & 2.0 & G & M & 33.0 & 5.8 & 10.8 \\
\hline $\mathrm{C} 3$ & FMC - FORD & FOCUS 3D & D & $\mathrm{F}$ & L4 & 2.0 & G & M & 33.0 & 5.8 & 10.8 \\
\hline $\mathrm{C} 3$ & FMC - FORD & FOCUS 3D & D & $\mathrm{F}$ & L4 & 2.0 & G & $A$ & 32.4 & 5.9 & 11.0 \\
\hline $\mathrm{C} 2$ & FMC - FORD & ESCORT ZX2 & $D$ & $\mathrm{~F}$ & L4 & 2.0 & G & $A$ & 33.1 & 5.8 & 10.8 \\
\hline $\mathrm{C} 7$ & FMC - FORD & FOCUS WAGON & $D$ & $\mathrm{~F}$ & L4 & 2.0 & G & A & 32.6 & 5.9 & 11.0 \\
\hline $\mathrm{C} 3$ & FMC - FORD & CONTOUR & $D$ & $\mathrm{~F}$ & L4 & 2.0 & G & M & 32.6 & 5.9 & 11.0 \\
\hline $\mathrm{C} 3$ & FMC - FORD & CONTOUR & $D$ & $\mathrm{~F}$ & L4 & 2.0 & G & A & 30.3 & 6.3 & 11.8 \\
\hline $\mathrm{C} 3$ & FMC - FORD & CONTOUR & $D$ & $\mathrm{~F}$ & L4 & 2.0 & G & A & 30.3 & 6.3 & 11.8 \\
\hline C3 & FMC - MAZDA & PROTEGE & I & $\mathrm{F}$ & L4 & 1.8 & G & A & 30.3 & 6.3 & 11.8 \\
\hline C3 & FMC - MAZDA & PROTEGE & I & $\mathrm{F}$ & L4 & 1.6 & G & A & 33.8 & 5.7 & 10.6 \\
\hline $\mathrm{C} 4$ & FMC - MAZDA & 626 & I & $\mathrm{F}$ & L4 & 2.0 & G & M & 33.5 & 5.7 & 10.6 \\
\hline C3 & FMC - MAZDA & PROTEGE & I & $\mathrm{F}$ & L4 & 1.8 & G & M & 32.4 & 5.9 & 11.0 \\
\hline $\mathrm{C} 6$ & FMC - MAZDA & MX-5 MIATA & I & $\mathrm{R}$ & L4 & 1.8 & G & M & 31.4 & 6.1 & 11.4 \\
\hline $\mathrm{C} 3$ & FMC - MAZDA & PROTEGE & I & $\mathrm{F}$ & L4 & 1.6 & G & M & 36.5 & 5.3 & 9.8 \\
\hline $\mathrm{C} 6$ & FMC - MAZDA & MX-5 MIATA & I & $\mathrm{R}$ & L4 & 1.8 & G & M & 30.2 & 6.3 & 11.8 \\
\hline C3 & FMC - MERCURY & MYSTIQUE & $\mathrm{D}$ & $\mathrm{F}$ & L4 & 2.0 & G & M & 32.4 & 5.9 & 11.0 \\
\hline
\end{tabular}




\begin{tabular}{|c|c|c|c|c|c|c|c|c|c|c|c|}
\hline $\begin{array}{l}\text { Type-Size } \\
\text { Class }\end{array}$ & Manufacturer/Division & Name & $\begin{array}{l}\text { Dom I } \\
\text { Imp }\end{array}$ & DR & Cyl & Disp & Fuel & Trans & MPG & GHG & $\begin{array}{l}\text { Oil } \\
\text { Use }\end{array}$ \\
\hline $\mathrm{C} 3$ & FMC - MERCURY & COUGAR & $\mathrm{D}$ & $\bar{F}$ & L4 & 2.0 & $\bar{G}$ & $\bar{M}$ & 32.4 & 5.9 & 11.0 \\
\hline C3 & FMC - MERCURY & MYSTIQUE & D & $\mathrm{F}$ & L4 & 2.0 & G & A & 29.6 & 6.5 & 12.1 \\
\hline $\mathrm{C} 2$ & GMC - CHEVY & METRO & I & $\mathrm{F}$ & L3 & 1.0 & G & M & 48.9 & 4.0 & 7.3 \\
\hline $\mathrm{C} 2$ & GMC - CHEVY & METRO & 1 & $\mathrm{~F}$ & L4 & 1.3 & G & M & 45.5 & 4.2 & 7.8 \\
\hline C3 & GMC - CHEVY & PRIZM & I & $\mathrm{F}$ & L4 & 1.8 & G & M & 39.6 & 4.9 & 9.0 \\
\hline C3 & GMC - CHEVY & PRIZM & I & $\mathrm{F}$ & L4 & 1.8 & G & A & 37.5 & 5.1 & 9.5 \\
\hline $\mathrm{C} 2$ & GMC - CHEVY & METRO & I & $\mathrm{F}$ & L4 & 1.3 & G & A & 37.6 & 5.1 & 9.5 \\
\hline C3 & GMC - CHEVY & PRIZM & I & $\mathrm{F}$ & L4 & 1.8 & G & A & 34.9 & 5.5 & 10.2 \\
\hline C2 & GMC - CHEVY & CAVALIER & D & $\mathrm{F}$ & L4 & 2.2 & G & M & 32.1 & 6.0 & 11.1 \\
\hline $\mathrm{C} 2$ & GMC - CHEVY & CAVALIER & D & $\mathrm{F}$ & L4 & 2.2 & G & A & 30.7 & 6.2 & 11.6 \\
\hline $\mathrm{C} 2$ & GMC - CHEVY & CAVALIER Z24 & D & $\mathrm{F}$ & L4 & 2.4 & G & M & 30.8 & 6.2 & 11.6 \\
\hline $\mathrm{C} 2$ & GMC - CHEVY & CAVALIER Z24 & D & $\mathrm{F}$ & L4 & 2.4 & G & A & 29.4 & 6.5 & 12.1 \\
\hline C3 & GMC - OLDSMOBILE & ALERO & D & $\mathrm{F}$ & L4 & 2.4 & G & M & 29.5 & 6.5 & 12.1 \\
\hline C3 & GMC - OLDSMOBILE & ALERO & D & $\mathrm{F}$ & L4 & 2.4 & G & A & 29.4 & 6.5 & 12.1 \\
\hline $\mathrm{C} 2$ & GMC - PONTIAC & SUNFIRE & D & $\mathrm{F}$ & L4 & 2.2 & G & M & 32.1 & 6.0 & 11.1 \\
\hline $\mathrm{C} 2$ & GMC - PONTIAC & SUNFIRE CNVT & D & $\mathrm{F}$ & L4 & 2.2 & G & A & 30.7 & 6.2 & 11.6 \\
\hline C2 & GMC - PONTIAC & SUNFIRE & D & $\mathrm{F}$ & L4 & 2.4 & G & M & 30.8 & 6.2 & 11.6 \\
\hline C3 & GMC - PONTIAC & GRAND AM & D & $\mathrm{F}$ & L4 & 2.4 & G & M & 29.5 & 6.5 & 12.1 \\
\hline $\mathrm{C} 2$ & GMC - PONTIAC & SUNFIRE & D & $\mathrm{F}$ & L4 & 2.4 & G & A & 29.4 & 6.5 & 12.1 \\
\hline C3 & GMC - PONTIAC & GRAND AM & D & $\mathrm{F}$ & L4 & 2.4 & G & A & 29.4 & 6.5 & 12.1 \\
\hline C3 & GMC - SATURN & SL & D & $\mathrm{F}$ & L4 & 1.9 & G & M & 38.7 & 5.0 & 9.2 \\
\hline $\mathrm{C} 2$ & GMC - SATURN & SC & D & $\mathrm{F}$ & L4 & 1.9 & G & M & 35.4 & 5.4 & 10.1 \\
\hline $\mathrm{C} 2$ & GMC - SATURN & SC & D & $\mathrm{F}$ & L4 & 1.9 & G & M & 38.1 & 5.0 & 9.4 \\
\hline C3 & GMC - SATURN & SL & D & $\mathrm{F}$ & L4 & 1.9 & G & M & 36.1 & 5.3 & 9.9 \\
\hline $\mathrm{C} 7$ & GMC - SATURN & SW & D & $\mathrm{F}$ & L4 & 1.9 & G & M & 36.1 & 5.3 & 9.9 \\
\hline C3 & GMC - SATURN & SL & D & $\mathrm{F}$ & L4 & 1.9 & G & A & 34.4 & 5.6 & 10.4 \\
\hline $\mathrm{C} 2$ & GMC - SATURN & SC & D & $\mathrm{F}$ & L4 & 1.9 & G & A & 33.9 & 5.7 & 10.5 \\
\hline $\mathrm{C} 7$ & GMC - SATURN & sW & D & $\mathrm{F}$ & L4 & 1.9 & G & A & 35.4 & 5.4 & 10.1 \\
\hline $\mathrm{C} 2$ & GMC - SATURN & sc & D & $\mathrm{F}$ & L4 & 1.9 & G & A & 36.1 & 5.3 & 9.9 \\
\hline C3 & GMC - SATURN & SL & D & $\mathrm{F}$ & L4 & 1.9 & G & A & 36.0 & 5.3 & 9.9 \\
\hline $\mathrm{C} 7$ & GMC - SATURN & sW & $\mathrm{D}$ & $F$ & L4 & 1.9 & G & A & 33.9 & 5.7 & 10.5 \\
\hline C4 & GMC - SATURN & LS & D & $\mathrm{F}$ & L4 & 2.2 & G & M & 31.9 & 6.0 & 11.2 \\
\hline C4 & GMC - SATURN & LS & D & $\mathrm{F}$ & L4 & 2.2 & G & A & 30.5 & 6.3 & 11.7 \\
\hline $\mathrm{C} 8$ & GMC - SATURN & LW & D & $\mathrm{F}$ & L4 & 2.2 & G & A & 29.4 & 6.5 & 12.1 \\
\hline $\mathrm{C} 2$ & HONDA - ACURA & ACURA INTEGRA & 1 & $\mathrm{~F}$ & L4 & 1.8 & G & M & 32.3 & 5.9 & 11.0 \\
\hline $\mathrm{C} 2$ & HONDA - ACURA & ACURA INTEGRA -R & 1 & $\mathrm{~F}$ & L4 & 1.8 & G & M & 31.9 & 6.0 & 11.2 \\
\hline $\mathrm{C} 2$ & HONDA - ACURA & ACURA INTEGRA & 1 & $\mathrm{~F}$ & L4 & 1.8 & G & A & 30.7 & 6.2 & 11.6 \\
\hline $\mathrm{C} 6$ & HONDA & INSIGHT & 1 & $\mathrm{~F}$ & L3 & 1.0 & G/EL & M & 76.3 & 2.6 & 4.7 \\
\hline C2 & HONDA & CIVIC HX & 1 & $\mathrm{~F}$ & L4 & 1.6 & G & M & 44.7 & 4.3 & 8.0 \\
\hline C2 & HONDA & CIVIC HX & 1 & $\mathrm{~F}$ & L4 & 1.6 & G & A & 41.9 & 4.6 & 8.5 \\
\hline C2 & HONDA & CIVIC $d x 4$ Dr & 1 & $\mathrm{~F}$ & L4 & 1.6 & G & M & 37.2 & 5.2 & 9.6 \\
\hline C2 & HONDA & CIVIC ex & 1 & $\mathrm{~F}$ & L4 & 1.6 & G & M & 37.2 & 5.2 & 9.6 \\
\hline C2 & HONDA & CIVIC vp & 1 & $\mathrm{~F}$ & L4 & 1.6 & G & A & 35.5 & 5.4 & 10.1 \\
\hline $\mathrm{C} 2$ & HONDA & CIVIC dx 4Dr & 1 & $\mathrm{~F}$ & L4 & 1.6 & G & A & 35.6 & 5.4 & 10.0 \\
\hline C2 & HONDA & CIVIC ex & 1 & $\mathrm{~F}$ & L4 & 1.6 & G & A & 35.5 & 5.4 & 10.1 \\
\hline C2 & HONDA & CIVIC si & 1 & $\mathrm{~F}$ & L4 & 1.6 & G & M & 32.8 & 5.8 & 10.9 \\
\hline C4 & HONDA & ACCORD Ix & 1 & $\mathrm{~F}$ & L4 & 2.3 & G & M & 32.1 & 6.0 & 11.1 \\
\hline C4 & HONDA & ACCORD & I & $\mathrm{F}$ & L4 & 2.3 & G & M & 30.4 & 6.3 & 11.8 \\
\hline C4 & HONDA & ACCORD IX & 1 & $\mathrm{~F}$ & L4 & 2.3 & G & A & 29.9 & 6.4 & 11.9 \\
\hline C3 & HYUNDAI & ACCENT & 1 & $\mathrm{~F}$ & L4 & 1.5 & G & M & 36.6 & 5.3 & 9.8 \\
\hline
\end{tabular}




\begin{tabular}{|c|c|c|c|c|c|c|c|c|c|c|c|}
\hline $\begin{array}{l}\text { Type-Size } \\
\text { Class }\end{array}$ & Manufacturer/Division & Name & $\begin{array}{l}\text { Dom I } \\
\text { Imp }\end{array}$ & DR & Cyl & Disp & Fuel & Trans & MPG & GHG & $\begin{array}{l}\text { Oil } \\
\text { Use }\end{array}$ \\
\hline $\mathrm{C} 3$ & HYUNDAI & ACCENT & 1 & $\mathrm{~F}$ & L4 & 1.5 & $G$ & $\mathrm{~A}$ & 34.2 & 5.6 & 10.4 \\
\hline C3 & HYUNDAI & ELANTRA & I & $\mathrm{F}$ & L4 & 2.0 & G & M & 32.1 & 6.0 & 11.1 \\
\hline $\mathrm{C} 7$ & HYUNDAI & ELANTRA WAGON & I & $\mathrm{F}$ & L4 & 2.0 & G & M & 31.3 & 6.1 & 11.4 \\
\hline $\mathrm{C} 2$ & HYUNDAI & TIBURON & 1 & $\mathrm{~F}$ & L4 & 2.0 & G & M & 31.1 & 6.1 & 11.5 \\
\hline C3 & $\mathrm{KIA}$ & SEPHIA L4 & 1 & $\mathrm{~F}$ & L4 & 1.8 & G & A & 29.9 & 6.4 & 12.0 \\
\hline C3 & $\mathrm{KIA}$ & SEPHIA M5 & 1 & $\mathrm{~F}$ & L4 & 1.8 & G & M & 29.5 & 6.5 & 12.1 \\
\hline $\mathrm{C} 2$ & MITSUBISHI & MIRAGE 2D & I & $\mathrm{F}$ & L4 & 1.5 & G & M & 41.7 & 4.6 & 8.6 \\
\hline $\mathrm{C} 2$ & MITSUBISHI & MIRAGE & I & $\mathrm{F}$ & L4 & 1.8 & G & M & 36.3 & 5.3 & 9.8 \\
\hline C2 & MITSUBISHI & MIRAGE 2D & I & $\mathrm{F}$ & L4 & 1.5 & G & A & 36.7 & 5.2 & 9.7 \\
\hline $\mathrm{C} 2$ & MITSUBISHI & MIRAGE & 1 & $\mathrm{~F}$ & L4 & 1.8 & G & A & 33.2 & 5.8 & 10.8 \\
\hline $\mathrm{C} 2$ & MITSUBISHI & ECLIPSE & 1 & $\mathrm{~F}$ & L4 & 2.4 & G & M & 30.1 & 6.3 & 11.9 \\
\hline $\mathrm{C} 2$ & MITSUBISHI & ECLIPSE & 1 & $\mathrm{~F}$ & L4 & 2.4 & G & A & 30.1 & 6.3 & 11.9 \\
\hline C3 & NISSAN - INFINITY & INFINITI G20 & 1 & $\mathrm{~F}$ & L4 & 2.0 & G & M & 31.0 & 6.2 & 11.5 \\
\hline C3 & NISSAN - INFINITY & INFINITI G20 & 1 & $\mathrm{~F}$ & L4 & 2.0 & G & A & 30.2 & 6.3 & 11.8 \\
\hline C3 & NISSAN & SENTRA & 1 & $\mathrm{~F}$ & L4 & 1.8 & G & M & 34.9 & 5.5 & 10.2 \\
\hline C3 & NISSAN & SENTRA & 1 & $\mathrm{~F}$ & L4 & 1.8 & G & A & 33.9 & 5.6 & 10.5 \\
\hline C3 & NISSAN & SENTRA & 1 & $\mathrm{~F}$ & L4 & 2.0 & G & M & 30.9 & 6.2 & 11.6 \\
\hline C3 & NISSAN & ALTIMA & 1 & $\mathrm{~F}$ & L4 & 2.4 & G & M & 30.9 & 6.2 & 11.6 \\
\hline C3 & NISSAN & SENTRA & 1 & $\mathrm{~F}$ & L4 & 2.0 & G & A & 30.8 & 6.2 & 11.6 \\
\hline $\mathrm{C} 2$ & SUZUKI & SWIFT & 1 & $\mathrm{~F}$ & L4 & 1.3 & G & M & 45.5 & 4.2 & 7.8 \\
\hline C2 & SUZUKI & ESTEEM & 1 & $\mathrm{~F}$ & L4 & 1.6 & G & M & 38.6 & 5.0 & 9.3 \\
\hline $\mathrm{C} 2$ & SUZUKI & SWIFT & 1 & $\mathrm{~F}$ & L4 & 1.3 & G & A & 37.6 & 5.1 & 9.5 \\
\hline $\mathrm{C} 2$ & SUZUKI & ESTEEM & I & $\mathrm{F}$ & L4 & 1.8 & G & M & 36.2 & 5.3 & 9.9 \\
\hline $\mathrm{C} 7$ & SUZUKI & ESTEEM WAGON & 1 & $\mathrm{~F}$ & L4 & 1.8 & G & M & 35.1 & 5.5 & 10.2 \\
\hline C2 & SUZUKI & ESTEEM & 1 & $\mathrm{~F}$ & L4 & 1.6 & G & A & 35.0 & 5.5 & 10.2 \\
\hline $\mathrm{C} 7$ & SUZUKI & ESTEEM WAGON & 1 & $\mathrm{~F}$ & L4 & 1.6 & G & A & 33.7 & 5.7 & 10.6 \\
\hline $\mathrm{C} 7$ & SUZUKI & ESTEEM WAGON & 1 & $\mathrm{~F}$ & L4 & 1.8 & G & A & 33.7 & 5.7 & 10.6 \\
\hline $\mathrm{C} 2$ & SUZUKI & ESTEEM & 1 & $\mathrm{~F}$ & L4 & 1.8 & G & A & 33.7 & 5.7 & 10.6 \\
\hline C4 & TOYOTA & CAMRY CNG & 1 & $\mathrm{~F}$ & L4 & 2.2 & NG & A & 30.2 & 5.4 & 1.8 \\
\hline C3 & TOYOTA & ECHO & 1 & $\mathrm{~F}$ & L4 & 1.5 & G & M & 43.1 & 4.5 & 8.3 \\
\hline C3 & TOYOTA & COROLLA & I & $\mathrm{F}$ & L4 & 1.8 & G & M & 39.6 & 4.9 & 9.0 \\
\hline C3 & TOYOTA & $\mathrm{ECHO}$ & 1 & $\mathrm{~F}$ & L4 & 1.5 & G & A & 39.4 & 4.9 & 9.1 \\
\hline C3 & TOYOTA & COROLLA & I & $\mathrm{F}$ & L4 & 1.8 & G & A & 37.4 & 5.1 & 9.6 \\
\hline C2 & TOYOTA & CELICA & 1 & $\mathrm{~F}$ & L4 & 1.8 & G & M & 35.2 & 5.4 & 10.1 \\
\hline $\mathrm{C} 2$ & TOYOTA & CELICA & 1 & $\mathrm{~F}$ & L4 & 1.8 & G & A & 34.9 & 5.5 & 10.2 \\
\hline C3 & TOYOTA & COROLLA & 1 & $\mathrm{~F}$ & L4 & 1.8 & G & A & 34.9 & 5.5 & 10.2 \\
\hline $\mathrm{C} 6$ & TOYOTA & MR2 & 1 & $\mathrm{~F}$ & L4 & 1.8 & G & M & 31.8 & 6.0 & 11.2 \\
\hline C3 & TOYOTA & CAMRY SOLARA & 1 & $\mathrm{~F}$ & L4 & 2.2 & G & M & 31.0 & 6.2 & 11.5 \\
\hline C4 & TOYOTA & CAMRY & I & $\mathrm{F}$ & L4 & 2.2 & G & M & 31.0 & 6.2 & 11.5 \\
\hline C2 & TOYOTA & CELICA & 1 & $\mathrm{~F}$ & L4 & 1.8 & G & M & 30.8 & 6.2 & 11.6 \\
\hline C2 & TOYOTA & CELICA & 1 & $\mathrm{~F}$ & L4 & 1.8 & G & A & 30.1 & 6.4 & 11.9 \\
\hline C3 & TOYOTA & CAMRY SOLARA & 1 & $\mathrm{~F}$ & L4 & 2.2 & G & A & 30.1 & 6.4 & 11.9 \\
\hline C4 & TOYOTA & CAMRY & 1 & $\mathrm{~F}$ & L4 & 2.2 & G & A & 30.1 & 6.4 & 11.9 \\
\hline $\mathrm{C} 2$ & TOYOTA & $\begin{array}{l}\text { CAMRY SOLARA } \\
\text { CONVERT }\end{array}$ & 1 & $\mathrm{~F}$ & L4 & 2.2 & G & A & 30.1 & 6.4 & 11.9 \\
\hline C3 & VWA - AUDI & AUDI A4 & 1 & $\mathrm{~F}$ & L4 & 1.8 & G & M & 31.9 & 6.0 & 11.2 \\
\hline C2 & VWA & NEW BEETLE & 1 & $\mathrm{~F}$ & L4 & 1.9 & D & M & 52.7 & 4.0 & 6.8 \\
\hline C3 & VWA & GOLF TDI & 1 & $\mathrm{~F}$ & L4 & 1.9 & D & M & 52.7 & 4.0 & 6.8 \\
\hline C3 & VWA & JETTA & 1 & $\mathrm{~F}$ & L4 & 1.9 & D & M & 52.7 & 4.0 & 6.8 \\
\hline C2 & VWA & NEW BEETLE & 1 & $\mathrm{~F}$ & L4 & 1.9 & D & A & 44.7 & 4.7 & 8.0 \\
\hline C3 & VWA & GOLF TDI & I & $\mathrm{F}$ & L4 & 1.9 & D & A & 44.7 & 4.7 & 8.0 \\
\hline
\end{tabular}




\begin{tabular}{|c|c|c|c|c|c|c|c|c|c|c|c|}
\hline $\begin{array}{l}\text { Type-Size } \\
\text { Class }\end{array}$ & Manufacturer/Division & Name & $\begin{array}{l}\text { Dom I } \\
\text { Imp }\end{array}$ & DR & Cyl & Disp & Fuel & Trans & MPG & GHG & $\begin{array}{l}\text { Oil } \\
\text { Use }\end{array}$ \\
\hline C3 & VWA & JETTA & 1 & $\mathrm{~F}$ & L4 & 1.9 & $\mathrm{D}$ & $A$ & 44.7 & 4.7 & 8.0 \\
\hline $\mathrm{C} 2$ & VWA & NEW BEETLE & I & $\mathrm{F}$ & L4 & 1.8 & G & M & 32.3 & 5.9 & 11.1 \\
\hline $\mathrm{C} 2$ & VWA & CABRIO & I & $\mathrm{F}$ & L4 & 2.0 & G & M & 31.2 & 6.1 & 11.5 \\
\hline $\mathrm{C} 2$ & VWA & NEW BEETLE & I & $\mathrm{F}$ & L4 & 2.0 & G & M & 31.2 & 6.1 & 11.5 \\
\hline C3 & VWA & GTI & I & $\mathrm{F}$ & L4 & 2.0 & G & $\mathrm{M}$ & 31.2 & 6.1 & 11.5 \\
\hline C3 & VWA & GTI & I & $\mathrm{F}$ & L4 & 1.8 & G & $\mathrm{M}$ & 31.3 & 6.1 & 11.4 \\
\hline C3 & VWA & GOLF & I & $\mathrm{F}$ & L4 & 1.8 & G & M & 31.3 & 6.1 & 11.4 \\
\hline C3 & VWA & JETTA & I & $\mathrm{F}$ & L4 & 1.8 & G & M & 31.3 & 6.1 & 11.4 \\
\hline C3 & VWA & GOLF & 1 & $\mathrm{~F}$ & L4 & 2.0 & G & M & 31.2 & 6.1 & 11.5 \\
\hline C3 & VWA & JETTA & I & $\mathrm{F}$ & L4 & 2.0 & G & M & 31.2 & 6.1 & 11.5 \\
\hline C4 & VWA & PASSAT & I & $\mathrm{F}$ & L4 & 1.8 & G & M & 31.3 & 6.1 & 11.4 \\
\hline C8 & VWA & PASSAT WAGON & I & $\mathrm{F}$ & L4 & 1.8 & G & M & 31.3 & 6.1 & 11.4 \\
\hline \multicolumn{12}{|l|}{ Trucks } \\
\hline T1 & FMC - FORD & RANGER 4X2 EV & $\mathrm{D}$ & $\mathrm{R}$ & E0 & 2.5 & $\mathrm{EL}$ & $\mathrm{A}$ & 29.7 & 5.2 & 1.8 \\
\hline T1 & GMC - CHEVROLET & S10 P/U 2WD & D & $\mathrm{R}$ & L4 & 2.2 & $\mathrm{FF}$ & M & 23.9 & 5.9 & 7.6 \\
\hline $\mathrm{T7}$ & GMC - CHEVROLET & $\begin{array}{l}\text { TRACKER CONV } \\
2 \text { WD }\end{array}$ & $\mathrm{D}$ & $\mathrm{R}$ & L4 & 1.6 & G & M & 31.1 & 6.2 & 11.5 \\
\hline T7 & GMC - CHEVROLET & $\begin{array}{l}\text { TRACKER CONV } \\
4 \mathrm{X} 4\end{array}$ & $\mathrm{D}$ & 4 & L4 & 1.6 & G & M & 30.7 & 6.2 & 11.6 \\
\hline T1 & GMC - CHEVROLET & S10 P/U 2WD & D & $\mathrm{R}$ & L4 & 2.2 & G & M & 29.6 & 6.5 & 12.1 \\
\hline T1 & GMC & SONOMA 2WD & I & $\mathrm{R}$ & L4 & 2.2 & FF & M & 23.9 & 5.9 & 7.6 \\
\hline T1 & GMC & SONOMA 2WD & 1 & $\mathrm{R}$ & L4 & 2.2 & G & M & 29.6 & 6.5 & 12.1 \\
\hline T1 & ISUZU & HOMBRE P/U 2WD & I & $\mathrm{R}$ & L4 & 2.2 & FF & M & 23.9 & 5.9 & 7.6 \\
\hline $\mathrm{T} 7$ & SUZUKI & VITARA CONV 2WD & I & $\mathrm{R}$ & L4 & 1.6 & G & $\mathrm{M}$ & 31.1 & 6.1 & 11.5 \\
\hline $\mathrm{T7}$ & SUZUKI & VITARA CONV 4WD & I & 4 & L4 & 1.6 & G & $\mathrm{M}$ & 30.7 & 6.2 & 11.6 \\
\hline $\mathrm{T7}$ & TOYOTA & RAV4 2WD & I & $\mathrm{F}$ & L4 & 2.0 & G & M & 30.9 & 6.2 & 11.6 \\
\hline T7 & TOYOTA & RAV4 2WD & I & $\mathrm{F}$ & L4 & 2.0 & G & A & 30.5 & 6.3 & 11.7 \\
\hline
\end{tabular}


Energy Star Method 3 Vehicle List

Top $20 \%$ of vehicles by Oil Use Score

\begin{tabular}{|c|c|c|c|c|c|c|c|c|c|c|c|}
\hline $\begin{array}{l}\text { Type- } \\
\text { Size } \\
\text { Class } \\
\end{array}$ & Manufacturer/Division & Name & $\begin{array}{c}\text { Dom I } \\
\text { Imp }\end{array}$ & DR & Cyl & Disp & Fuel & Trans & MPG & GHG & Oil Use \\
\hline \multicolumn{12}{|l|}{ Cars } \\
\hline $\mathrm{C} 4$ & DAEWOO & LANOS- 3Dr & I & $\mathrm{F}$ & L4 & 1.5 & G & $\mathrm{M}$ & 34.8 & 5.5 & 10.3 \\
\hline $\mathrm{C} 4$ & DAEWOO & LANOS-3Dr & 1 & $\mathrm{~F}$ & L4 & 1.6 & G & M & 34.6 & 5.5 & 10.3 \\
\hline $\mathrm{C} 4$ & DAEWOO & LANOS- 3Dr & I & $\mathrm{F}$ & L4 & 1.5 & G & $A$ & 32.9 & 5.8 & 10.8 \\
\hline $\mathrm{C} 4$ & DAEWOO & LANOS-4Dr & 1 & $\mathrm{~F}$ & L4 & 1.6 & G & M & 31.8 & 6.0 & 11.2 \\
\hline $\mathrm{C} 4$ & DAEWOO & LANOS-3Dr & I & $\mathrm{F}$ & L4 & 1.6 & G & $A$ & 31.2 & 6.1 & 11.4 \\
\hline $\mathrm{C} 4$ & DAEWOO & NUBIRA & I & $\mathrm{F}$ & L4 & 2.0 & G & M & 30.1 & 6.3 & 11.8 \\
\hline $\mathrm{C} 7$ & DAEWOO & NUBIRA WAGON & I & $\mathrm{F}$ & L4 & 2.0 & G & M & 30.1 & 6.3 & 11.8 \\
\hline $\mathrm{C} 7$ & DAEWOO & NUBIRA WAGON & 1 & $\mathrm{~F}$ & L4 & 4.0 & G & M & 30.1 & 6.3 & 11.8 \\
\hline $\mathrm{C} 4$ & DAEWOO & NUBIRA & 1 & $\mathrm{~F}$ & L4 & 2.0 & G & M & 30.1 & 6.3 & 11.8 \\
\hline $\mathrm{C} 3$ & DCC - CHRYSLER & NEON & D & $\mathrm{F}$ & L4 & 2.0 & G & M & 36.1 & 5.3 & 9.9 \\
\hline $\mathrm{C} 4$ & DCC - CHRYSLER & CIRRUS & D & $\mathrm{F}$ & L4 & 2.0 & G & M & 34.3 & 5.6 & 10.4 \\
\hline C3 & DCC - CHRYSLER & NEON & $\mathrm{D}$ & $\mathrm{F}$ & L4 & 2.0 & G & A & 31.9 & 6.0 & 11.2 \\
\hline C3 & DCC - DODGE & NEON & $\mathrm{D}$ & $\mathrm{F}$ & L4 & 2.0 & G & M & 36.1 & 5.3 & 9.9 \\
\hline C3 & DCC - DODGE & NEON & $\mathrm{D}$ & $\mathrm{F}$ & L4 & 2.0 & G & A & 31.9 & 6.0 & 11.2 \\
\hline $\mathrm{C} 3$ & DCC - PLYMOUTH & NEON & $\mathrm{D}$ & $\mathrm{F}$ & L4 & 2.0 & G & M & 36.1 & 5.3 & 9.9 \\
\hline $\mathrm{C} 4$ & DCC - PLYMOUTH & BREEZE & $\mathrm{D}$ & $\mathrm{F}$ & L4 & 2.0 & G & M & 35.4 & 5.4 & 10.1 \\
\hline $\mathrm{C} 3$ & DCC - PLYMOUTH & NEON & $\mathrm{D}$ & $\mathrm{F}$ & L4 & 2.0 & G & $A$ & 31.9 & 6.0 & 11.2 \\
\hline C5 & FMC - FORD & $\begin{array}{l}\text { CROWN VICTORIA } \\
\text { NG }\end{array}$ & $\mathrm{D}$ & $\mathrm{R}$ & V8 & 4.6 & NG & A & 20.7 & 7.6 & 2.6 \\
\hline C5 & FMC - FORD & TAURUS FFV & $\mathrm{D}$ & $\mathrm{F}$ & V6 & 3.0 & $\mathrm{FF}$ & A & 24.6 & 5.9 & 7.8 \\
\hline C8 & FMC - FORD & TAURUS WAGON FFV & $\mathrm{D}$ & $\mathrm{F}$ & V6 & 3.0 & $\mathrm{FF}$ & $A$ & 23.3 & 6.2 & 8.3 \\
\hline $\mathrm{C} 7$ & FMC - FORD & FOCUS WAGON & $\mathrm{D}$ & $\mathrm{F}$ & L4 & 2.0 & G & M & 36.2 & 5.3 & 9.9 \\
\hline C3 & FMC - FORD & FOCUS & $\mathrm{D}$ & $\mathrm{F}$ & L4 & 2.0 & G & M & 35.7 & 5.4 & 10.0 \\
\hline C3 & FMC - FORD & ESCORT & $\mathrm{D}$ & $\mathrm{F}$ & L4 & 2.0 & G & M & 35.7 & 5.4 & 10.0 \\
\hline $\mathrm{C} 3$ & FMC - FORD & FOCUS & $\mathrm{D}$ & $\mathrm{F}$ & L4 & 2.0 & G & $A$ & 33.9 & 5.7 & 10.5 \\
\hline $\mathrm{C} 7$ & FMC - FORD & FOCUS WAGON & $\mathrm{D}$ & $\mathrm{F}$ & L4 & 2.0 & G & $A$ & 32.6 & 5.9 & 11.0 \\
\hline C3 & FMC - FORD & ESCORT & $\mathrm{D}$ & $\mathrm{F}$ & L4 & 2.0 & G & $A$ & 33.7 & 5.7 & 10.6 \\
\hline $\mathrm{C} 2$ & FMC - FORD & ESCORT ZX2 & $\mathrm{D}$ & $\mathrm{F}$ & L4 & 2.0 & G & M & 33.0 & 5.8 & 10.8 \\
\hline C3 & FMC - FORD & FOCUS 3D & $\mathrm{D}$ & $\mathrm{F}$ & L4 & 2.0 & G & M & 33.0 & 5.8 & 10.8 \\
\hline C3 & FMC - FORD & FOCUS 3D & $\mathrm{D}$ & $\mathrm{F}$ & L4 & 2.0 & G & A & 32.4 & 5.9 & 11.0 \\
\hline $\mathrm{C} 2$ & FMC - FORD & ESCORT ZX2 & D & $\mathrm{F}$ & L4 & 2.0 & G & $A$ & 33.1 & 5.8 & 10.8 \\
\hline $\mathrm{C} 7$ & FMC - FORD & FOCUS WAGON & $\mathrm{D}$ & $\mathrm{F}$ & L4 & 2.0 & G & $A$ & 32.6 & 5.9 & 11.0 \\
\hline C3 & FMC - FORD & CONTOUR & $\mathrm{D}$ & $\mathrm{F}$ & L4 & 2.0 & G & M & 32.6 & 5.9 & 11.0 \\
\hline C3 & FMC - FORD & CONTOUR & $\mathrm{D}$ & $\mathrm{F}$ & L4 & 2.0 & G & A & 30.3 & 6.3 & 11.8 \\
\hline $\mathrm{C} 3$ & FMC - FORD & CONTOUR & D & $\mathrm{F}$ & L4 & 2.0 & G & A & 30.3 & 6.3 & 11.8 \\
\hline $\mathrm{C} 3$ & FMC - MAZDA & PROTEGE & 1 & $\mathrm{~F}$ & L4 & 1.8 & G & A & 30.3 & 6.3 & 11.8 \\
\hline C3 & FMC - MAZDA & PROTEGE & 1 & $\mathrm{~F}$ & L4 & 1.6 & G & A & 33.8 & 5.7 & 10.6 \\
\hline $\mathrm{C} 4$ & FMC - MAZDA & 626 & 1 & $\mathrm{~F}$ & L4 & 2.0 & G & M & 33.5 & 5.7 & 10.6 \\
\hline C3 & FMC - MAZDA & PROTEGE & 1 & $\mathrm{~F}$ & L4 & 1.8 & G & M & 32.4 & 5.9 & 11.0 \\
\hline C6 & FMC - MAZDA & MX-5 MIATA & 1 & $\mathrm{R}$ & L4 & 1.8 & G & M & 31.4 & 6.1 & 11.4 \\
\hline C3 & FMC - MAZDA & PROTEGE & 1 & $\mathrm{~F}$ & L4 & 1.6 & G & M & 36.5 & 5.3 & 9.8 \\
\hline C6 & FMC - MAZDA & MX-5 MIATA & 1 & $\mathrm{R}$ & L4 & 1.8 & G & M & 30.2 & 6.3 & 11.8 \\
\hline C3 & FMC - MERCURY & MYSTIQUE & D & $\mathrm{F}$ & L4 & 2.0 & G & M & 32.4 & 5.9 & 11.0 \\
\hline C3 & FMC - MERCURY & COUGAR & D & $\mathrm{F}$ & L4 & 2.0 & G & M & 32.4 & 5.9 & 11.0 \\
\hline
\end{tabular}




\begin{tabular}{|c|c|c|c|c|c|c|c|c|c|c|c|}
\hline $\begin{array}{l}\text { Type- } \\
\text { Size } \\
\text { Class }\end{array}$ & Manufacturer/Division & Name & $\begin{array}{c}\text { Dom I } \\
\text { Imp }\end{array}$ & DR & Cyl & Disp & Fuel & Trans & MPG & GHG & Oil Use \\
\hline$\overline{\mathrm{C} 2}$ & GMC - CHEVY & METRO & $\mathrm{I}$ & $\mathrm{F}$ & L3 & 1.0 & $\mathrm{G}$ & $\mathrm{M}$ & 48.9 & 4.0 & 7.3 \\
\hline C2 & GMC - CHEVY & METRO & I & $\mathrm{F}$ & L4 & 1.3 & G & $\mathrm{M}$ & 45.5 & 4.2 & 7.8 \\
\hline C3 & GMC - CHEVY & PRIZM & I & $\mathrm{F}$ & L4 & 1.8 & G & M & 39.6 & 4.9 & 9.0 \\
\hline C3 & GMC - CHEVY & PRIZM & I & $\mathrm{F}$ & $\mathrm{L} 4$ & 1.8 & G & A & 37.5 & 5.1 & 9.5 \\
\hline $\mathrm{C} 2$ & GMC - CHEVY & METRO & I & $\mathrm{F}$ & L4 & 1.3 & G & A & 37.6 & 5.1 & 9.5 \\
\hline C3 & GMC - CHEVY & PRIZM & I & $\mathrm{F}$ & L4 & 1.8 & G & A & 34.9 & 5.5 & 10.2 \\
\hline C2 & GMC - CHEVY & CAVALIER & $\mathrm{D}$ & $\mathrm{F}$ & $\mathrm{L} 4$ & 2.2 & G & $\mathrm{M}$ & 32.1 & 6.0 & 11.1 \\
\hline C2 & GMC - CHEVY & CAVALIER & $\mathrm{D}$ & $\mathrm{F}$ & L4 & 2.2 & G & A & 30.7 & 6.2 & 11.6 \\
\hline C2 & GMC - CHEVY & CAVALIER Z24 & $\mathrm{D}$ & $\mathrm{F}$ & $\mathrm{L} 4$ & 2.4 & G & $M$ & 30.8 & 6.2 & 11.6 \\
\hline C2 & GMC - PONTIAC & SUNFIRE & D & $\mathrm{F}$ & L4 & 2.2 & G & M & 32.1 & 6.0 & 11.1 \\
\hline C2 & GMC - PONTIAC & SUNFIRE CNVT & $\mathrm{D}$ & $\mathrm{F}$ & L4 & 2.2 & G & A & 30.7 & 6.2 & 11.6 \\
\hline C2 & GMC - PONTIAC & SUNFIRE & $\mathrm{D}$ & $\mathrm{F}$ & $\mathrm{L} 4$ & 2.4 & G & M & 30.8 & 6.2 & 11.6 \\
\hline C3 & GMC - SATURN & SL & $\mathrm{D}$ & $\mathrm{F}$ & $\mathrm{L} 4$ & 1.9 & G & M & 38.7 & 5.0 & 9.2 \\
\hline C2 & GMC - SATURN & SC & $\mathrm{D}$ & $\mathrm{F}$ & L4 & 1.9 & G & $\mathrm{M}$ & 35.4 & 5.4 & 10.1 \\
\hline C2 & GMC - SATURN & SC & $\mathrm{D}$ & $\mathrm{F}$ & L4 & 1.9 & G & M & 38.1 & 5.0 & 9.4 \\
\hline C3 & GMC - SATURN & SL & $\mathrm{D}$ & $\mathrm{F}$ & L4 & 1.9 & G & $M$ & 36.1 & 5.3 & 9.9 \\
\hline $\mathrm{C} 7$ & GMC - SATURN & SW & $\mathrm{D}$ & $\mathrm{F}$ & $\mathrm{L} 4$ & 1.9 & G & $\mathrm{M}$ & 36.1 & 5.3 & 9.9 \\
\hline C3 & GMC - SATURN & SL & $\mathrm{D}$ & $\mathrm{F}$ & L4 & 1.9 & G & A & 34.4 & 5.6 & 10.4 \\
\hline C2 & GMC - SATURN & SC & $\mathrm{D}$ & $\mathrm{F}$ & $\mathrm{L} 4$ & 1.9 & G & A & 33.9 & 5.7 & 10.5 \\
\hline C7 & GMC - SATURN & SW & $\mathrm{D}$ & $\mathrm{F}$ & L4 & 1.9 & G & A & 35.4 & 5.4 & 10.1 \\
\hline C2 & GMC - SATURN & SC & $\mathrm{D}$ & $\mathrm{F}$ & L4 & 1.9 & G & A & 36.1 & 5.3 & 9.9 \\
\hline C3 & GMC - SATURN & SL & $\mathrm{D}$ & $\mathrm{F}$ & L4 & 1.9 & G & A & 36.0 & 5.3 & 9.9 \\
\hline $\mathrm{C} 7$ & GMC - SATURN & SW & $\mathrm{D}$ & $\mathrm{F}$ & L4 & 1.9 & G & A & 33.9 & 5.7 & 10.5 \\
\hline C4 & GMC - SATURN & LS & $\mathrm{D}$ & $\mathrm{F}$ & L4 & 2.2 & G & $M$ & 31.9 & 6.0 & 11.2 \\
\hline C4 & GMC - SATURN & LS & $\mathrm{D}$ & $\mathrm{F}$ & L4 & 2.2 & G & A & 30.5 & 6.3 & 11.7 \\
\hline $\mathrm{C} 2$ & HONDA - ACURA & ACURA INTEGRA & 1 & $\mathrm{~F}$ & L4 & 1.8 & G & M & 32.3 & 5.9 & 11.0 \\
\hline $\mathrm{C} 2$ & HONDA - ACURA & ACURA INTEGRA -R & 1 & $\mathrm{~F}$ & $\mathrm{~L} 4$ & 1.8 & G & M & 31.9 & 6.0 & 11.2 \\
\hline C2 & HONDA - ACURA & ACURA INTEGRA & 1 & $\mathrm{~F}$ & L4 & 1.8 & G & A & 30.7 & 6.2 & 11.6 \\
\hline C6 & HONDA & INSIGHT & I & $\mathrm{F}$ & L3 & 1.0 & $\mathrm{G} / \mathrm{EL}$ & M & 76.3 & 2.6 & 4.7 \\
\hline C2 & HONDA & CIVIC HX & I & $\mathrm{F}$ & L4 & 1.6 & G & M & 44.7 & 4.3 & 8.0 \\
\hline C2 & HONDA & CIVIC HX & I & $\mathrm{F}$ & L4 & 1.6 & G & A & 41.9 & 4.6 & 8.5 \\
\hline C2 & HONDA & CIVIC dx 4Dr & I & $\mathrm{F}$ & L4 & 1.6 & G & M & 37.2 & 5.2 & 9.6 \\
\hline C2 & HONDA & CIVIC ex & I & $\mathrm{F}$ & L4 & 1.6 & G & $\mathrm{M}$ & 37.2 & 5.2 & 9.6 \\
\hline C2 & HONDA & CIVIC vp & I & $\mathrm{F}$ & L4 & 1.6 & G & A & 35.5 & 5.4 & 10.1 \\
\hline C2 & HONDA & CIVIC dx 4Dr & I & $\mathrm{F}$ & L4 & 1.6 & G & $A$ & 35.6 & 5.4 & 10.0 \\
\hline C2 & HONDA & CIVIC ex & I & $\mathrm{F}$ & L4 & 1.6 & G & $A$ & 35.5 & 5.4 & 10.1 \\
\hline C2 & HONDA & CIVIC si & I & $\mathrm{F}$ & L4 & 1.6 & G & M & 32.8 & 5.8 & 10.9 \\
\hline C4 & HONDA & ACCORD Ix & I & $\mathrm{F}$ & L4 & 2.3 & G & M & 32.1 & 6.0 & 11.1 \\
\hline C4 & HONDA & ACCORD & I & $\mathrm{F}$ & L4 & 2.3 & G & $\mathrm{M}$ & 30.4 & 6.3 & 11.8 \\
\hline C3 & HYUNDAI & ACCENT & I & $\mathrm{F}$ & L4 & 1.5 & G & M & 36.6 & 5.3 & 9.8 \\
\hline C3 & HYUNDAI & ACCENT & I & $\mathrm{F}$ & L4 & 1.5 & G & A & 34.2 & 5.6 & 10.4 \\
\hline C3 & HYUNDAI & ELANTRA & I & $\mathrm{F}$ & L4 & 2.0 & G & $\mathrm{M}$ & 32.1 & 6.0 & 11.1 \\
\hline $\mathrm{C} 7$ & HYUNDAI & ELANTRA WAGON & I & $\mathrm{F}$ & L4 & 2.0 & G & M & 31.3 & 6.1 & 11.4 \\
\hline C2 & HYUNDAI & TIBURON & I & $\mathrm{F}$ & L4 & 2.0 & G & M & 31.1 & 6.1 & 11.5 \\
\hline C2 & MITSUBISHI & MIRAGE 2D & I & $\mathrm{F}$ & $\mathrm{L} 4$ & 1.5 & G & $\mathrm{M}$ & 41.7 & 4.6 & 8.6 \\
\hline $\mathrm{C} 2$ & MITSUBISHI & MIRAGE & I & $\mathrm{F}$ & L4 & 1.8 & G & $\mathrm{M}$ & 36.3 & 5.3 & 9.8 \\
\hline C2 & MITSUBISHI & MIRAGE 2D & I & $\mathrm{F}$ & L4 & 1.5 & G & A & 36.7 & 5.2 & 9.7 \\
\hline
\end{tabular}




\begin{tabular}{|c|c|c|c|c|c|c|c|c|c|c|c|}
\hline $\begin{array}{l}\text { Type- } \\
\text { Size } \\
\text { Class } \\
\mathrm{C} 2\end{array}$ & $\begin{array}{l}\text { Manufacturer/Division } \\
\text { MITSUBISHI }\end{array}$ & $\begin{array}{l}\text { Name } \\
\text { MIRAGE }\end{array}$ & $\frac{\begin{array}{c}\text { Dom / } \\
\text { Imp }\end{array}}{1}$ & $\frac{\mathrm{DR}}{\mathrm{F}}$ & $\frac{\text { Cyl }}{\text { L4 }}$ & $\begin{array}{r}\text { Disp } \\
1.8\end{array}$ & $\frac{\text { Fuel }}{G}$ & $\frac{\text { Trans }}{A}$ & $\frac{\text { MPG }}{33.2}$ & $\begin{array}{r}\text { GHG } \\
5.8\end{array}$ & $\frac{\text { Oil Use }}{10.8}$ \\
\hline C2 & MITSUBISHI & ECLIPSE & I & $\mathrm{F}$ & L4 & 2.4 & G & M & 30.1 & 6.3 & 11.9 \\
\hline $\mathrm{C} 2$ & MITSUBISHI & ECLIPSE & 1 & $\mathrm{~F}$ & L4 & 2.4 & G & A & 30.1 & 6.3 & 11.9 \\
\hline C3 & NISSAN - INFINITY & INFINITI G20 & I & $\mathrm{F}$ & L4 & 2.0 & G & M & 31.0 & 6.2 & 11.5 \\
\hline C3 & NISSAN - INFINITY & INFINITI G20 & 1 & $\mathrm{~F}$ & L4 & 2.0 & G & A & 30.2 & 6.3 & 11.8 \\
\hline C3 & NISSAN & SENTRA & 1 & $\mathrm{~F}$ & L4 & 1.8 & G & M & 34.9 & 5.5 & 10.2 \\
\hline C3 & NISSAN & SENTRA & 1 & $\mathrm{~F}$ & L4 & 1.8 & G & A & 33.9 & 5.6 & 10.5 \\
\hline C3 & NISSAN & SENTRA & 1 & $\mathrm{~F}$ & L4 & 2.0 & G & M & 30.9 & 6.2 & 11.6 \\
\hline C3 & NISSAN & ALTIMA & 1 & $\mathrm{~F}$ & L4 & 2.4 & G & M & 30.9 & 6.2 & 11.6 \\
\hline C3 & NISSAN & SENTRA & 1 & $\mathrm{~F}$ & L4 & 2.0 & G & A & 30.8 & 6.2 & 11.6 \\
\hline $\mathrm{C} 2$ & SUZUKI & SWIFT & 1 & $\mathrm{~F}$ & L4 & 1.3 & G & M & 45.5 & 4.2 & 7.8 \\
\hline $\mathrm{C} 2$ & SUZUKI & ESTEEM & 1 & $\mathrm{~F}$ & L4 & 1.6 & G & M & 38.6 & 5.0 & 9.3 \\
\hline C2 & SUZUKI & SWIFT & 1 & $\mathrm{~F}$ & L4 & 1.3 & G & A & 37.6 & 5.1 & 9.5 \\
\hline C2 & SUZUKI & ESTEEM & 1 & $\mathrm{~F}$ & L4 & 1.8 & G & M & 36.2 & 5.3 & 9.9 \\
\hline C7 & SUZUKI & ESTEEM WAGON & 1 & $\mathrm{~F}$ & L4 & 1.8 & G & M & 35.1 & 5.5 & 10.2 \\
\hline $\mathrm{C} 2$ & SUZUKI & ESTEEM & 1 & $\mathrm{~F}$ & L4 & 1.6 & G & A & 35.0 & 5.5 & 10.2 \\
\hline $\mathrm{C} 7$ & SUZUKI & ESTEEM WAGON & 1 & $\mathrm{~F}$ & L4 & 1.6 & G & A & 33.7 & 5.7 & 10.6 \\
\hline $\mathrm{C} 7$ & SUZUKI & ESTEEM WAGON & 1 & $\mathrm{~F}$ & L4 & 1.8 & G & A & 33.7 & 5.7 & 10.6 \\
\hline C2 & SUZUKI & ESTEEM & 1 & $\mathrm{~F}$ & L4 & 1.8 & G & A & 33.7 & 5.7 & 10.6 \\
\hline C4 & TOYOTA & CAMRY CNG & 1 & $\mathrm{~F}$ & L4 & 2.2 & NG & A & 30.2 & 5.4 & 1.8 \\
\hline C3 & TOYOTA & $\mathrm{ECHO}$ & 1 & $\mathrm{~F}$ & L4 & 1.5 & G & M & 43.1 & 4.5 & 8.3 \\
\hline C3 & TOYOTA & COROLLA & 1 & $\mathrm{~F}$ & L4 & 1.8 & G & M & 39.6 & 4.9 & 9.0 \\
\hline C3 & TOYOTA & $\mathrm{ECHO}$ & 1 & $\mathrm{~F}$ & L4 & 1.5 & G & A & 39.4 & 4.9 & 9.1 \\
\hline C3 & TOYOTA & COROLLA & 1 & $\mathrm{~F}$ & L4 & 1.8 & G & A & 37.4 & 5.1 & 9.6 \\
\hline $\mathrm{C} 2$ & TOYOTA & CELICA & 1 & $\mathrm{~F}$ & L4 & 1.8 & G & M & 35.2 & 5.4 & 10.1 \\
\hline C2 & TOYOTA & CELICA & 1 & $\mathrm{~F}$ & L4 & 1.8 & G & A & 34.9 & 5.5 & 10.2 \\
\hline C3 & TOYOTA & COROLLA & 1 & $\mathrm{~F}$ & L4 & 1.8 & G & A & 34.9 & 5.5 & 10.2 \\
\hline C6 & TOYOTA & MR2 & 1 & $\mathrm{~F}$ & L4 & 1.8 & G & M & 31.8 & 6.0 & 11.2 \\
\hline C3 & TOYOTA & CAMRY SOLARA & 1 & $\mathrm{~F}$ & L4 & 2.2 & G & M & 31.0 & 6.2 & 11.5 \\
\hline C4 & TOYOTA & CAMRY & 1 & $\mathrm{~F}$ & L4 & 2.2 & G & M & 31.0 & 6.2 & 11.5 \\
\hline C2 & TOYOTA & CELICA & 1 & $\mathrm{~F}$ & L4 & 1.8 & G & M & 30.8 & 6.2 & 11.6 \\
\hline C3 & VWA - AUDI & AUDI A4 & 1 & $\mathrm{~F}$ & L4 & 1.8 & G & M & 31.9 & 6.0 & 11.2 \\
\hline $\mathrm{C} 2$ & VWA & NEW BEETLE & 1 & $\mathrm{~F}$ & L4 & 1.9 & D & M & 52.7 & 4.0 & 6.8 \\
\hline C3 & VWA & GOLF TDI & 1 & $\mathrm{~F}$ & L4 & 1.9 & D & M & 52.7 & 4.0 & 6.8 \\
\hline C3 & VWA & JETTA & 1 & $\mathrm{~F}$ & L4 & 1.9 & D & M & 52.7 & 4.0 & 6.8 \\
\hline $\mathrm{C} 2$ & VWA & NEW BEETLE & 1 & $\mathrm{~F}$ & L4 & 1.9 & D & A & 44.7 & 4.7 & 8.0 \\
\hline C3 & VWA & GOLF TDI & 1 & $\mathrm{~F}$ & L4 & 1.9 & $\mathrm{D}$ & A & 44.7 & 4.7 & 8.0 \\
\hline C3 & VWA & JETTA & 1 & $\mathrm{~F}$ & L4 & 1.9 & D & A & 44.7 & 4.7 & 8.0 \\
\hline C2 & VWA & NEW BEETLE & 1 & $\mathrm{~F}$ & L4 & 1.8 & G & M & 32.3 & 5.9 & 11.1 \\
\hline C2 & VWA & CABRIO & 1 & $\mathrm{~F}$ & L4 & 2.0 & G & M & 31.2 & 6.1 & 11.5 \\
\hline C2 & VWA & NEW BEETLE & 1 & $\mathrm{~F}$ & L4 & 2.0 & G & M & 31.2 & 6.1 & 11.5 \\
\hline C3 & VWA & GTI & 1 & $\mathrm{~F}$ & L4 & 2.0 & G & M & 31.2 & 6.1 & 11.5 \\
\hline C3 & VWA & GTI & 1 & $\mathrm{~F}$ & L4 & 1.8 & G & M & 31.3 & 6.1 & 11.4 \\
\hline C3 & VWA & GOLF & 1 & $\mathrm{~F}$ & L4 & 1.8 & G & M & 31.3 & 6.1 & 11.4 \\
\hline C3 & VWA & JETTA & 1 & $\mathrm{~F}$ & L4 & 1.8 & G & M & 31.3 & 6.1 & 11.4 \\
\hline C3 & VWA & GOLF & I & $\mathrm{F}$ & L4 & 2.0 & G & M & 31.2 & 6.1 & 11.5 \\
\hline C3 & VWA & JETTA & 1 & $\mathrm{~F}$ & L4 & 2.0 & G & M & 31.2 & 6.1 & 11.5 \\
\hline
\end{tabular}




\begin{tabular}{|c|c|c|c|c|c|c|c|c|c|c|c|}
\hline $\begin{array}{l}\text { Type- } \\
\text { Size } \\
\text { Class }\end{array}$ & Manufacturer/Division & Name & $\begin{array}{l}\text { Dom I } \\
\text { Imp }\end{array}$ & DR & Cyl & Disp & Fuel & Trans & MPG & GHG & Oil Use \\
\hline$\overline{\mathrm{C} 4}$ & VWA & PASSAT & $\mathrm{I}$ & $\mathrm{F}$ & $\mathrm{L} 4$ & 1.8 & $G$ & $\bar{M}$ & 31.3 & 6.1 & $\overline{11.4}$ \\
\hline $\mathrm{C} 8$ & VWA & PASSAT WAGON & I & $\mathrm{F}$ & L4 & 1.8 & G & M & 31.3 & 6.1 & 11.4 \\
\hline \multicolumn{12}{|l|}{ Trucks } \\
\hline T3 & DCC - CHRYSLER & $\begin{array}{l}\text { TOWN \& CNTRY 2WD } \\
\text { FFV }\end{array}$ & $\mathrm{D}$ & $\mathrm{F}$ & V6 & 3.3 & $\mathrm{FF}$ & $A$ & 20.0 & 7.0 & 9.1 \\
\hline T4 & DCC - DODGE & B2500 WAGON & $\mathrm{D}$ & $\mathrm{R}$ & V8 & 5.2 & NG & A & 15.2 & 10.2 & 3.5 \\
\hline T4 & DCC - DODGE & B2500 VAN & $\mathrm{D}$ & $\mathrm{R}$ & V8 & 5.2 & NG & A & 15.5 & 10.0 & 3.5 \\
\hline T3 & DCC - DODGE & CARAVAN 2WD FFV & $\mathrm{D}$ & $\mathrm{F}$ & V6 & 3.3 & $\mathrm{FF}$ & A & 19.9 & 7.1 & 9.2 \\
\hline T3 & DCC - PLYMOUTH & $\begin{array}{l}\text { CARAVAN/VOYAGER } \\
\text { 2WD }\end{array}$ & $\mathrm{D}$ & $\mathrm{F}$ & V6 & 3.3 & FF & A & 19.9 & 7.1 & 9.2 \\
\hline T1 & FMC - FORD & RANGER 4X2 EV & $\mathrm{D}$ & $\mathrm{R}$ & E0 & 2.5 & EL & A & 29.7 & 5.2 & 1.8 \\
\hline $\mathrm{T} 2$ & FMC - FORD & F150 4X2 NGV & $\mathrm{D}$ & $\mathrm{R}$ & V8 & 5.4 & NG & A & 15.8 & 9.9 & 3.4 \\
\hline $\mathrm{T} 4$ & FMC - FORD & E250 VAN NGV & $\mathrm{D}$ & $\mathrm{R}$ & V8 & 5.4 & NG & A & 15.5 & 10.1 & 3.5 \\
\hline T1 & FMC - FORD & RANGER 4X2 FFV & $\mathrm{D}$ & $\mathrm{R}$ & V6 & 3.0 & $\mathrm{FF}$ & M & 19.7 & 7.1 & 9.2 \\
\hline T1 & FMC - FORD & RANGER 4X4 FFV & $\mathrm{D}$ & 4 & V6 & 3.0 & $\mathrm{FF}$ & M & 19.2 & 7.3 & 9.5 \\
\hline T1 & FMC - FORD & RANGER 4X2 FFV & $\mathrm{D}$ & $\mathrm{R}$ & V6 & 3.0 & FF & A & 18.5 & 7.6 & 9.7 \\
\hline T1 & FMC - FORD & RANGER 4X4 FFV & $\mathrm{D}$ & 4 & V6 & 3.0 & FF & A & 17.7 & 8.0 & 10.4 \\
\hline T1 & GMC - CHEVROLET & S10 P/U 2WD & $\mathrm{D}$ & $\mathrm{R}$ & $\mathrm{L} 4$ & 2.2 & $\mathrm{FF}$ & $M$ & 23.9 & 5.9 & 7.6 \\
\hline T1 & GMC - CHEVROLET & S10 P/U 2WD & $\mathrm{D}$ & $\mathrm{R}$ & L4 & 2.2 & $\mathrm{FF}$ & A & 21.2 & 6.7 & 8.7 \\
\hline $\mathrm{T} 7$ & GMC - CHEVROLET & TRACKER CONV 2WD & $\mathrm{D}$ & $\mathrm{R}$ & $\mathrm{L} 4$ & 1.6 & G & M & 31.1 & 6.2 & 11.5 \\
\hline T7 & GMC - CHEVROLET & TRACKER CONV 4X4 & $\mathrm{D}$ & 4 & L4 & 1.6 & G & M & 30.7 & 6.2 & 11.6 \\
\hline T1 & GMC & SONOMA 2WD & 1 & $\mathrm{R}$ & L4 & 2.2 & FF & M & 23.9 & 5.9 & 7.6 \\
\hline T1 & GMC & SONOMA 2WD & I & $\mathrm{R}$ & L4 & 2.2 & $\mathrm{FF}$ & A & 21.2 & 6.7 & 8.7 \\
\hline T1 & ISUZU & HOMBRE P/U 2WD & I & $\mathrm{R}$ & L4 & 2.2 & $\mathrm{FF}$ & M & 23.9 & 5.9 & 7.6 \\
\hline T1 & ISUZU & HOMBRE P/U 2WD & I & $\mathrm{R}$ & $\mathrm{L} 4$ & 2.2 & $\mathrm{FF}$ & A & 21.2 & 6.7 & 8.7 \\
\hline $\mathrm{T} 7$ & SUZUKI & VITARA CONV 2WD & I & $\mathrm{R}$ & $\mathrm{L} 4$ & 1.6 & G & $M$ & 31.1 & 6.1 & 11.5 \\
\hline $\mathrm{T} 7$ & SUZUKI & VITARA CONV 4WD & I & 4 & L4 & 1.6 & G & $M$ & 30.7 & 6.2 & 11.6 \\
\hline T1 & TKM - MAZDA & MAZDA 4X2 FFV & I & $\mathrm{R}$ & V6 & 3.0 & $\mathrm{FF}$ & M & 19.7 & 7.1 & 9.2 \\
\hline $\mathrm{T} 1$ & TKM - MAZDA & MAZDA 4X4 FFV & I & 4 & V6 & 3.0 & $\mathrm{FF}$ & $M$ & 19.2 & 7.3 & 9.5 \\
\hline T1 & TKM - MAZDA & MAZDA 4X2 FFV & I & $\mathrm{R}$ & V6 & 3.0 & $\mathrm{FF}$ & $A$ & 18.5 & 7.6 & 9.7 \\
\hline T1 & TKM - MAZDA & MAZDA 4X4 FFV & I & 4 & V6 & 3.0 & FF & $A$ & 17.7 & 8.0 & 10.4 \\
\hline T7 & TOYOTA & RAV4 2WD & I & $\mathrm{F}$ & L4 & 2.0 & G & $M$ & 30.9 & 6.2 & 11.6 \\
\hline $\mathrm{T} 7$ & TOYOTA & RAV4 2WD & I & $\mathrm{F}$ & L4 & 2.0 & G & $A$ & 30.5 & 6.3 & 11.7 \\
\hline
\end{tabular}


Energy Star Method 4 Vehicle List

Top $20 \%$ of vehicles by GHG \& Oil Use Score

\begin{tabular}{|c|c|c|c|c|c|c|c|c|c|c|c|}
\hline $\begin{array}{l}\text { Type-Size } \\
\text { Class } \\
\end{array}$ & Manufacturer/Division & Name & $\begin{array}{c}\text { Dom I } \\
\text { Imp }\end{array}$ & DR & Cyl & Disp & Fuel & Trans & MPG & GHG & Oil Use \\
\hline \multicolumn{12}{|l|}{ Cars } \\
\hline $\mathrm{C} 4$ & DAEWOO & LANOS- 3Dr & 1 & $F$ & $\mathrm{~L} 4$ & 1.5 & G & $\mathrm{M}$ & 34.8 & 5.5 & 10.3 \\
\hline C4 & DAEWOO & LANOS-3Dr & 1 & $\mathrm{~F}$ & L4 & 1.6 & G & M & 34.6 & 5.5 & 10.3 \\
\hline C4 & DAEWOO & LANOS- 3Dr & 1 & $\mathrm{~F}$ & L4 & 1.5 & G & A & 32.9 & 5.8 & 10.8 \\
\hline C4 & DAEWOO & LANOS-4Dr & 1 & $\mathrm{~F}$ & L4 & 1.6 & G & M & 31.8 & 6.0 & 11.2 \\
\hline C4 & DAEWOO & LANOS-3Dr & I & $\mathrm{F}$ & L4 & 1.6 & G & A & 31.2 & 6.1 & 11.4 \\
\hline C4 & DAEWOO & NUBIRA & I & $\mathrm{F}$ & L4 & 2.0 & G & M & 30.1 & 6.3 & 11.8 \\
\hline $\mathrm{C} 7$ & DAEWOO & NUBIRA WAGON & I & $\mathrm{F}$ & L4 & 2.0 & G & M & 30.1 & 6.3 & 11.8 \\
\hline $\mathrm{C} 7$ & DAEWOO & NUBIRA WAGON & 1 & $F$ & L4 & 4.0 & G & M & 30.1 & 6.3 & 11.8 \\
\hline C4 & DAEWOO & NUBIRA & 1 & $F$ & L4 & 2.0 & G & M & 30.1 & 6.3 & 11.8 \\
\hline C3 & DCC - CHRYSLER & NEON & D & $F$ & L4 & 2.0 & G & M & 36.1 & 5.3 & 9.9 \\
\hline C4 & DCC - CHRYSLER & CIRRUS & D & $\mathrm{F}$ & L4 & 2.0 & G & M & 34.3 & 5.6 & 10.4 \\
\hline C3 & DCC - CHRYSLER & NEON & $\mathrm{D}$ & $\mathrm{F}$ & L4 & 2.0 & G & A & 31.9 & 6.0 & 11.2 \\
\hline C3 & DCC - DODGE & NEON & $\mathrm{D}$ & $\mathrm{F}$ & L4 & 2.0 & G & M & 36.1 & 5.3 & 9.9 \\
\hline C3 & DCC - DODGE & NEON & $\mathrm{D}$ & $\mathrm{F}$ & L4 & 2.0 & G & A & 31.9 & 6.0 & 11.2 \\
\hline C3 & DCC - PLYMOUTH & NEON & $\mathrm{D}$ & $\mathrm{F}$ & L4 & 2.0 & G & M & 36.1 & 5.3 & 9.9 \\
\hline C4 & DCC - PLYMOUTH & BREEZE & $\mathrm{D}$ & $\mathrm{F}$ & L4 & 2.0 & G & M & 35.4 & 5.4 & 10.1 \\
\hline C3 & DCC - PLYMOUTH & NEON & $\mathrm{D}$ & $\mathrm{F}$ & L4 & 2.0 & G & A & 31.9 & 6.0 & 11.2 \\
\hline C5 & FMC - FORD & CROWN VICTORIA NG & $\mathrm{D}$ & $\mathrm{R}$ & V8 & 4.6 & NG & A & 20.7 & 7.6 & 2.6 \\
\hline C5 & FMC - FORD & TAURUS FFV & $\mathrm{D}$ & $\mathrm{F}$ & V6 & 3.0 & FF & A & 24.6 & 5.9 & 7.8 \\
\hline C8 & FMC - FORD & TAURUS WAGON FFV & $\mathrm{D}$ & $\mathrm{F}$ & V6 & 3.0 & $\mathrm{FF}$ & A & 23.3 & 6.2 & 8.3 \\
\hline $\mathrm{C} 7$ & FMC - FORD & FOCUS WAGON & $\mathrm{D}$ & $\mathrm{F}$ & L4 & 2.0 & G & M & 36.2 & 5.3 & 9.9 \\
\hline C3 & FMC - FORD & FOCUS & $\mathrm{D}$ & $\mathrm{F}$ & L4 & 2.0 & G & $\mathrm{M}$ & 35.7 & 5.4 & 10.0 \\
\hline C3 & FMC - FORD & ESCORT & $\mathrm{D}$ & $\mathrm{F}$ & L4 & 2.0 & G & M & 35.7 & 5.4 & 10.0 \\
\hline C3 & FMC - FORD & FOCUS & $\mathrm{D}$ & $\mathrm{F}$ & L4 & 2.0 & G & A & 33.9 & 5.7 & 10.5 \\
\hline $\mathrm{C} 7$ & FMC - FORD & FOCUS WAGON & $\mathrm{D}$ & $\mathrm{F}$ & L4 & 2.0 & G & A & 32.6 & 5.9 & 11.0 \\
\hline C3 & FMC - FORD & ESCORT & $\mathrm{D}$ & $F$ & L4 & 2.0 & G & $A$ & 33.7 & 5.7 & 10.6 \\
\hline $\mathrm{C} 2$ & FMC - FORD & ESCORT ZX2 & $\mathrm{D}$ & $F$ & L4 & 2.0 & G & M & 33.0 & 5.8 & 10.8 \\
\hline C3 & FMC - FORD & FOCUS 3D & $\mathrm{D}$ & $\mathrm{F}$ & L4 & 2.0 & G & M & 33.0 & 5.8 & 10.8 \\
\hline C3 & FMC - FORD & FOCUS 3D & $\mathrm{D}$ & $\mathrm{F}$ & L4 & 2.0 & G & A & 32.4 & 5.9 & 11.0 \\
\hline $\mathrm{C} 2$ & FMC - FORD & ESCORT ZX2 & $\mathrm{D}$ & $F$ & L4 & 2.0 & G & A & 33.1 & 5.8 & 10.8 \\
\hline $\mathrm{C} 7$ & FMC - FORD & FOCUS WAGON & $\mathrm{D}$ & $\mathrm{F}$ & L4 & 2.0 & G & A & 32.6 & 5.9 & 11.0 \\
\hline C3 & FMC - FORD & CONTOUR & $\mathrm{D}$ & $\mathrm{F}$ & L4 & 2.0 & G & $\mathrm{M}$ & 32.6 & 5.9 & 11.0 \\
\hline C3 & FMC - FORD & CONTOUR & $\mathrm{D}$ & $\mathrm{F}$ & L4 & 2.0 & G & A & 30.3 & 6.3 & 11.8 \\
\hline C3 & FMC - FORD & CONTOUR & $\mathrm{D}$ & $\mathrm{F}$ & L4 & 2.0 & G & A & 30.3 & 6.3 & 11.8 \\
\hline C3 & FMC - MAZDA & PROTEGE & 1 & $F$ & L4 & 1.8 & G & $A$ & 30.3 & 6.3 & 11.8 \\
\hline C3 & FMC - MAZDA & PROTEGE & 1 & $\mathrm{~F}$ & L4 & 1.6 & G & A & 33.8 & 5.7 & 10.6 \\
\hline C4 & FMC - MAZDA & 626 & I & $\mathrm{F}$ & L4 & 2.0 & G & M & 33.5 & 5.7 & 10.6 \\
\hline C3 & FMC - MAZDA & PROTEGE & 1 & $\mathrm{~F}$ & L4 & 1.8 & G & M & 32.4 & 5.9 & 11.0 \\
\hline C6 & FMC - MAZDA & MX-5 MIATA & 1 & $\mathrm{R}$ & L4 & 1.8 & G & M & 31.4 & 6.1 & 11.4 \\
\hline C3 & FMC - MAZDA & PROTEGE & 1 & $\mathrm{~F}$ & L4 & 1.6 & G & $M$ & 36.5 & 5.3 & 9.8 \\
\hline C6 & FMC - MAZDA & MX-5 MIATA & I & $\mathrm{R}$ & L4 & 1.8 & G & M & 30.2 & 6.3 & 11.8 \\
\hline C3 & FMC - MERCURY & MYSTIQUE & $\mathrm{D}$ & $\mathrm{F}$ & L4 & 2.0 & G & M & 32.4 & 5.9 & 11.0 \\
\hline C3 & FMC - MERCURY & COUGAR & D & $\mathrm{F}$ & L4 & 2.0 & G & $M$ & 32.4 & 5.9 & 11.0 \\
\hline
\end{tabular}




\begin{tabular}{|c|c|c|c|c|c|c|c|c|c|c|c|}
\hline $\begin{array}{l}\text { Type-Size } \\
\text { Class }\end{array}$ & Manufacturer/Division & Name & $\begin{array}{c}\text { Dom I } \\
\text { Imp }\end{array}$ & DR & Cyl & Disp & Fuel & Trans & MPG & GHG & Oil Use \\
\hline$\overline{\mathrm{C} 2}$ & GMC - CHEVY & METRO & $\mathrm{I}$ & $\bar{F}$ & L3 & 1.0 & $\mathrm{G}$ & $\bar{M}$ & 48.9 & 4.0 & $\overline{7.3}$ \\
\hline $\mathrm{C} 2$ & GMC - CHEVY & METRO & I & $\mathrm{F}$ & L4 & 1.3 & G & M & 45.5 & 4.2 & 7.8 \\
\hline C3 & GMC - CHEVY & PRIZM & 1 & $\mathrm{~F}$ & L4 & 1.8 & G & M & 39.6 & 4.9 & 9.0 \\
\hline C3 & GMC - CHEVY & PRIZM & I & $\mathrm{F}$ & L4 & 1.8 & G & $A$ & 37.5 & 5.1 & 9.5 \\
\hline $\mathrm{C} 2$ & GMC - CHEVY & METRO & I & $\mathrm{F}$ & $\mathrm{L} 4$ & 1.3 & G & $A$ & 37.6 & 5.1 & 9.5 \\
\hline C3 & GMC - CHEVY & PRIZM & 1 & $\mathrm{~F}$ & L4 & 1.8 & G & A & 34.9 & 5.5 & 10.2 \\
\hline C2 & GMC - CHEVY & CAVALIER & $\mathrm{D}$ & $\mathrm{F}$ & L4 & 2.2 & G & M & 32.1 & 6.0 & 11.1 \\
\hline $\mathrm{C} 2$ & GMC - CHEVY & CAVALIER & $\mathrm{D}$ & $F$ & L4 & 2.2 & G & $A$ & 30.7 & 6.2 & 11.6 \\
\hline C2 & GMC - CHEVY & CAVALIER Z24 & $\mathrm{D}$ & $\mathrm{F}$ & L4 & 2.4 & G & M & 30.8 & 6.2 & 11.6 \\
\hline $\mathrm{C} 2$ & GMC - PONTIAC & SUNFIRE & $\mathrm{D}$ & $\mathrm{F}$ & L4 & 2.2 & G & M & 32.1 & 6.0 & 11.1 \\
\hline C2 & GMC - PONTIAC & SUNFIRE CNVT & D & $\mathrm{F}$ & L4 & 2.2 & G & $A$ & 30.7 & 6.2 & 11.6 \\
\hline C2 & GMC - PONTIAC & SUNFIRE & $\mathrm{D}$ & $\mathrm{F}$ & L4 & 2.4 & G & M & 30.8 & 6.2 & 11.6 \\
\hline C3 & GMC - SATURN & SL & $\mathrm{D}$ & $\mathrm{F}$ & L4 & 1.9 & G & M & 38.7 & 5.0 & 9.2 \\
\hline C2 & GMC - SATURN & $\mathrm{SC}$ & $\mathrm{D}$ & $\mathrm{F}$ & L4 & 1.9 & G & M & 35.4 & 5.4 & 10.1 \\
\hline $\mathrm{C} 2$ & GMC - SATURN & SC & $\mathrm{D}$ & $\mathrm{F}$ & L4 & 1.9 & G & $M$ & 38.1 & 5.0 & 9.4 \\
\hline C3 & GMC - SATURN & SL & $\mathrm{D}$ & $\mathrm{F}$ & $\mathrm{L} 4$ & 1.9 & G & M & 36.1 & 5.3 & 9.9 \\
\hline $\mathrm{C7}$ & GMC - SATURN & SW & $\mathrm{D}$ & $\mathrm{F}$ & L4 & 1.9 & G & M & 36.1 & 5.3 & 9.9 \\
\hline C3 & GMC - SATURN & SL & $\mathrm{D}$ & $\mathrm{F}$ & Ł4 & 1.9 & G & $A$ & 34.4 & 5.6 & 10.4 \\
\hline C2 & GMC - SATURN & $\mathrm{SC}$ & $\mathrm{D}$ & $\mathrm{F}$ & L4 & 1.9 & G & $A$ & 33.9 & 5.7 & 10.5 \\
\hline $\mathrm{C} 7$ & GMC - SATURN & sw & $\mathrm{D}$ & $\mathrm{F}$ & L4 & 1.9 & G & A & 35.4 & 5.4 & 10.1 \\
\hline C2 & GMC - SATURN & SC & $\mathrm{D}$ & $\mathrm{F}$ & L4 & 1.9 & G & $A$ & 36.1 & 5.3 & 9.9 \\
\hline C3 & GMC - SATURN & SL & $\mathrm{D}$ & $\mathrm{F}$ & L4 & 1.9 & G & A & 36.0 & 5.3 & 9.9 \\
\hline $\mathrm{C7}$ & GMC - SATURN & SW & $\mathrm{D}$ & $\mathrm{F}$ & L4 & 1.9 & G & $A$ & 33.9 & 5.7 & 10.5 \\
\hline $\mathrm{C} 4$ & GMC - SATURN & LS & $\mathrm{D}$ & $\mathrm{F}$ & L4 & 2.2 & G & M & 31.9 & 6.0 & 11.2 \\
\hline C4 & GMC - SATURN & LS & $\mathrm{D}$ & $\mathrm{F}$ & L4 & 2.2 & G & $A$ & 30.5 & 6.3 & 11.7 \\
\hline $\mathrm{C} 2$ & HONDA - ACURA & ACURA INTEGRA & I & $\mathrm{F}$ & L4 & 1.8 & G & M & 32.3 & 5.9 & 11.0 \\
\hline C2 & HONDA - ACURA & ACURA INTEGRA -R & 1 & $\mathrm{~F}$ & L4 & 1.8 & G & M & 31.9 & 6.0 & 11.2 \\
\hline C2 & HONDA - ACURA & ACURA INTEGRA & I & $\mathrm{F}$ & L4 & 1.8 & G & $A$ & 30.7 & 6.2 & 11.6 \\
\hline C6 & HONDA & INSIGHT & I & $\mathrm{F}$ & L3 & 1.0 & $\mathrm{G} / \mathrm{EL}$ & M & 76.3 & 2.6 & 4.7 \\
\hline $\mathrm{C} 2$ & HONDA & CIVIC HX & I & $\mathrm{F}$ & L4 & 1.6 & G & $M$ & 44.7 & 4.3 & 8.0 \\
\hline C2 & HONDA & CIVIC HX & I & $\mathrm{F}$ & L4 & 1.6 & G & A & 41.9 & 4.6 & 8.5 \\
\hline C2 & HONDA & CIVIC dx 4Dr & 1 & $\mathrm{~F}$ & Ł4 & 1.6 & G & M & 37.2 & 5.2 & 9.6 \\
\hline C2 & HONDA & CIVIC ex & I & $\mathrm{F}$ & L4 & 1.6 & G & $M$ & 37.2 & 5.2 & 9.6 \\
\hline C2 & HONDA & CIVIC vp & I & $\mathrm{F}$ & L4 & 1.6 & G & A & 35.5 & 5.4 & 10.1 \\
\hline C2 & HONDA & CIVIC dx 4Dr & I & $\mathrm{F}$ & L4 & 1.6 & G & $A$ & 35.6 & 5.4 & 10.0 \\
\hline C2 & HONDA & CIVIC ex & 1 & $\mathrm{~F}$ & L4 & 1.6 & G & $A$ & 35.5 & 5.4 & 10.1 \\
\hline C2 & HONDA & CIVIC si & I & $\mathrm{F}$ & L4 & 1.6 & G & $M$ & 32.8 & 5.8 & 10.9 \\
\hline C4 & HONDA & ACCORD Ix & I & $\mathrm{F}$ & L4 & 2.3 & G & $M$ & 32.1 & 6.0 & 11.1 \\
\hline C4 & HONDA & ACCORD & I & $\mathrm{F}$ & L4 & 2.3 & G & M & 30.4 & 6.3 & 11.8 \\
\hline C3 & HYUNDAI & ACCENT & I & $\mathrm{F}$ & L4 & 1.5 & G & M & 36.6 & 5.3 & 9.8 \\
\hline C3 & HYUNDAI & ACCENT & I & $\mathrm{F}$ & L4 & 1.5 & G & A & 34.2 & 5.6 & 10.4 \\
\hline C3 & HYUNDAI & ELANTRA & I & $\mathrm{F}$ & Ł4 & 2.0 & G & M & 32.1 & 6.0 & 11.1 \\
\hline $\mathrm{C7}$ & HYUNDAI & ELANTRA WAGON & I & $\mathrm{F}$ & L4 & 2.0 & G & $M$ & 31.3 & 6.1 & 11.4 \\
\hline $\mathrm{C} 2$ & HYUNDAI & TIBURON & 1 & $F$ & L4 & 2.0 & G & M & 31.1 & 6.1 & 11.5 \\
\hline C2 & MITSUBISHI & MIRAGE 2D & I & $\mathrm{F}$ & L4 & 1.5 & G & $M$ & 41.7 & 4.6 & 8.6 \\
\hline C2 & MITSUBISHI & MIRAGE & I & $\mathrm{F}$ & L4 & 1.8 & G & M & 36.3 & 5.3 & 9.8 \\
\hline $\mathrm{C} 2$ & MITSUBISHI & MIRAGE 2D & I & $\mathrm{F}$ & L4 & 1.5 & G & $A$ & 36.7 & 5.2 & 9.7 \\
\hline
\end{tabular}




\begin{tabular}{|c|c|c|c|c|c|c|c|c|c|c|c|}
\hline $\begin{array}{l}\text { Type-Size } \\
\text { Class }\end{array}$ & Manufacturer/Division & Name & $\begin{array}{l}\text { Dom I } \\
\text { Imp }\end{array}$ & DR & Cyl & Disp & Fuel & Trans & MPG & GHG & Oil Use \\
\hline$\overline{\mathrm{C} 2}$ & MITSUBISHI & MIRAGE & $\mathrm{I}$ & $\bar{F}$ & $\mathrm{~L} 4$ & 1.8 & $\mathrm{G}$ & $\mathrm{A}$ & 33.2 & 5.8 & 10.8 \\
\hline C2 & MITSUBISHI & ECLIPSE & 1 & $\mathrm{~F}$ & L4 & 2.4 & G & M & 30.1 & 6.3 & 11.9 \\
\hline $\mathrm{C} 2$ & MITSUBISHI & ECLIPSE & I & $F$ & L4 & 2.4 & G & A & 30.1 & 6.3 & 11.9 \\
\hline C3 & NISSAN - INFINITY & INFINITI G20 & 1 & $F$ & L4 & 2.0 & G & M & 31.0 & 6.2 & 11.5 \\
\hline C3 & NISSAN - INFINITY & INFINITI G20 & 1 & $F$ & L4 & 2.0 & G & A & 30.2 & 6.3 & 11.8 \\
\hline C3 & NISSAN & SENTRA & 1 & $F$ & L4 & 1.8 & G & M & 34.9 & 5.5 & 10.2 \\
\hline C3 & NISSAN & SENTRA & 1 & $\mathrm{~F}$ & L4 & 1.8 & G & A & 33.9 & 5.6 & 10.5 \\
\hline C3 & NISSAN & SENTRA & I & $F$ & L4 & 2.0 & G & M & 30.9 & 6.2 & 11.6 \\
\hline C3 & NISSAN & ALTIMA & 1 & $\mathrm{~F}$ & L4 & 2.4 & G & M & 30.9 & 6.2 & 11.6 \\
\hline C3 & NISSAN & SENTRA & 1 & $\mathrm{~F}$ & L4 & 2.0 & G & A & 30.8 & 6.2 & 11.6 \\
\hline $\mathrm{C} 2$ & SUZUKI & SWIFT & 1 & $F$ & L4 & 1.3 & G & M & 45.5 & 4.2 & 7.8 \\
\hline $\mathrm{C} 2$ & SUZUKI & ESTEEM & 1 & $F$ & L4 & 1.6 & G & M & 38.6 & 5.0 & 9.3 \\
\hline $\mathrm{C} 2$ & SUZUKI & SWIFT & 1 & $F$ & L4 & 1.3 & G & A & 37.6 & 5.1 & 9.5 \\
\hline $\mathrm{C} 2$ & SUZUKI & ESTEEM & 1 & $F$ & L4 & 1.8 & G & M & 36.2 & 5.3 & 9.9 \\
\hline $\mathrm{C} 7$ & SUZUKI & ESTEEM WAGON & 1 & $\mathrm{~F}$ & L4 & 1.8 & G & M & 35.1 & 5.5 & 10.2 \\
\hline C2 & SUZUKI & ESTEEM & I & $\mathrm{F}$ & L4 & 1.6 & G & A & 35.0 & 5.5 & 10.2 \\
\hline C7 & SUZUKI & ESTEEM WAGON & 1 & $F$ & L4 & 1.6 & G & A & 33.7 & 5.7 & 10.6 \\
\hline $\mathrm{C} 7$ & SUZUKI & ESTEEM WAGON & 1 & $F$ & L4 & 1.8 & G & $A$ & 33.7 & 5.7 & 10.6 \\
\hline $\mathrm{C} 2$ & SUZUKI & ESTEEM & 1 & $F$ & L4 & 1.8 & G & A & 33.7 & 5.7 & 10.6 \\
\hline C4 & TOYOTA & CAMRY CNG & 1 & $\mathrm{~F}$ & L4 & 2.2 & NG & A & 30.2 & 5.4 & 1.8 \\
\hline C3 & TOYOTA & $\mathrm{ECHO}$ & 1 & $\mathrm{~F}$ & L4 & 1.5 & G & M & 43.1 & 4.5 & 8.3 \\
\hline C3 & TOYOTA & COROLLA & 1 & $\mathrm{~F}$ & L4 & 1.8 & G & M & 39.6 & 4.9 & 9.0 \\
\hline C3 & TOYOTA & $\mathrm{ECHO}$ & 1 & $F$ & L4 & 1.5 & G & A & 39.4 & 4.9 & 9.1 \\
\hline C3 & TOYOTA & COROLLA & 1 & $F$ & L4 & 1.8 & G & A & 37.4 & 5.1 & 9.6 \\
\hline $\mathrm{C} 2$ & TOYOTA & CELICA & 1 & $\mathrm{~F}$ & L4 & 1.8 & G & M & 35.2 & 5.4 & 10.1 \\
\hline C2 & TOYOTA & CELICA & 1 & $\mathrm{~F}$ & L4 & 1.8 & G & A & 34.9 & 5.5 & 10.2 \\
\hline C3 & TOYOTA & COROLLA & 1 & $\mathrm{~F}$ & L4 & 1.8 & G & A & 34.9 & 5.5 & 10.2 \\
\hline $\mathrm{C} 6$ & TOYOTA & MR2 & 1 & $\mathrm{~F}$ & L4 & 1.8 & G & M & 31.8 & 6.0 & 11.2 \\
\hline C3 & TOYOTA & CAMRY SOLARA & 1 & $F$ & L4 & 2.2 & G & M & 31.0 & 6.2 & 11.5 \\
\hline C4 & TOYOTA & CAMRY & 1 & $\mathrm{~F}$ & L4 & 2.2 & G & M & 31.0 & 6.2 & 11.5 \\
\hline $\mathrm{C} 2$ & TOYOTA & CELICA & 1 & $\mathrm{~F}$ & L4 & 1.8 & G & M & 30.8 & 6.2 & 11.6 \\
\hline $\mathrm{C} 2$ & TOYOTA & CELICA & 1 & $\mathrm{~F}$ & L4 & 1.8 & G & A & 30.1 & 6.4 & 11.9 \\
\hline C3 & TOYOTA & CAMRY SOLARA & 1 & $F$ & L4 & 2.2 & G & A & 30.1 & 6.4 & 11.9 \\
\hline C4 & TOYOTA & CAMRY & 1 & $\mathrm{~F}$ & L4 & 2.2 & G & A & 30.1 & 6.4 & 11.9 \\
\hline C2 & TOYOTA & $\begin{array}{l}\text { CAMRY SOLARA } \\
\text { CONVERT }\end{array}$ & I & $\mathrm{F}$ & L4 & 2.2 & G & $A$ & 30.1 & 6.4 & 11.9 \\
\hline C3 & VWA - AUDI & AUDI A4 & 1 & $\mathrm{~F}$ & L4 & 1.8 & G & $M$ & 31.9 & 6.0 & 11.2 \\
\hline C2 & VWA & NEW BEETLE & I & $F$ & L4 & 1.9 & $\mathrm{D}$ & M & 52.7 & 4.0 & 6.8 \\
\hline C3 & VWA & GOLF TDI & 1 & $F$ & L4 & 1.9 & D & M & 52.7 & 4.0 & 6.8 \\
\hline C3 & VWA & JETTA & 1 & $\mathrm{~F}$ & L4 & 1.9 & D & M & 52.7 & 4.0 & 6.8 \\
\hline C2 & VWA & NEW BEETLE & 1 & $\mathrm{~F}$ & L4 & 1.9 & D & A & 44.7 & 4.7 & 8.0 \\
\hline C3 & VWA & GOLF TDI & 1 & $\mathrm{~F}$ & L4 & 1.9 & D & A & 44.7 & 4.7 & 8.0 \\
\hline C3 & VWA & JETTA & 1 & $F$ & L4 & 1.9 & D & A & 44.7 & 4.7 & 8.0 \\
\hline C2 & VWA & NEW BEETLE & 1 & $\mathrm{~F}$ & L4 & 1.8 & G & $\mathrm{M}$ & 32.3 & 5.9 & 11.1 \\
\hline C2 & VWA & CABRIO & 1 & $\mathrm{~F}$ & L4 & 2.0 & G & M & 31.2 & 6.1 & 11.5 \\
\hline C2 & VWA & NEW BEETLE & I & $F$ & L4 & 2.0 & G & M & 31.2 & 6.1 & 11.5 \\
\hline C3 & VWA & GTI & I & $\mathrm{F}$ & L4 & 2.0 & G & $M$ & 31.2 & 6.1 & 11.5 \\
\hline
\end{tabular}




\begin{tabular}{|c|c|c|c|c|c|c|c|c|c|c|c|}
\hline $\begin{array}{l}\text { Type-Size } \\
\text { Class }\end{array}$ & Manufacturer/Division & Name & $\begin{array}{l}\text { Dom I } \\
\text { Imp }\end{array}$ & DR & Cyl & Disp & Fuel & Trans & MPG & GHG & Oil Use \\
\hline C3 & VWA & GTI & I & $\mathrm{F}$ & $\mathrm{L} 4$ & 1.8 & G & $M$ & 31.3 & 6.1 & 11.4 \\
\hline $\mathrm{C} 3$ & VWA & GOLF & 1 & $\mathrm{~F}$ & L4 & 1.8 & G & M & 31.3 & 6.1 & 11.4 \\
\hline $\mathrm{C} 3$ & VWA & JETTA & I & $\mathrm{F}$ & L4 & 1.8 & G & M & 31.3 & 6.1 & 11.4 \\
\hline $\mathrm{C} 3$ & VWA & GOLF & 1 & $\mathrm{~F}$ & L4 & 2.0 & G & M & 31.2 & 6.1 & 11.5 \\
\hline C3 & VWA & JETTA & I & $\mathrm{F}$ & L4 & 2.0 & G & M & 31.2 & 6.1 & 11.5 \\
\hline $\mathrm{C} 4$ & VWA & PASSAT & 1 & $\mathrm{~F}$ & L4 & 1.8 & G & M & 31.3 & 6.1 & 11.4 \\
\hline $\mathrm{C} 8$ & VWA & PASSAT WAGON & I & $\mathrm{F}$ & L4 & 1.8 & G & M & 31.3 & 6.1 & 11.4 \\
\hline \multicolumn{12}{|l|}{ Trucks } \\
\hline T3 & DCC - CHRYSLER & $\begin{array}{l}\text { TOWN \& CNTRY 2WD } \\
\text { FFV }\end{array}$ & $\mathrm{D}$ & $F$ & V6 & 3.3 & $\mathrm{FF}$ & A & 20.0 & 7.0 & 9.1 \\
\hline $\mathrm{T} 4$ & DCC - DODGE & B2500 WAGON & $\mathrm{D}$ & $\mathrm{R}$ & V8 & 5.2 & NG & $A$ & 15.2 & 10.2 & 3.5 \\
\hline T4 & DCC - DODGE & B2500 VAN & $\mathrm{D}$ & $\mathrm{R}$ & V8 & 5.2 & NG & A & 15.5 & 10.0 & 3.5 \\
\hline T3 & DCC - DODGE & CARAVAN 2WD FFV & $\mathrm{D}$ & $\mathrm{F}$ & V6 & 3.3 & $\mathrm{FF}$ & $A$ & 19.9 & 7.1 & 9.2 \\
\hline T3 & DCC - PLYMOUTH & $\begin{array}{l}\text { CARAVAN/VOYAGER } \\
\text { 2WD }\end{array}$ & $\mathrm{D}$ & $\mathrm{F}$ & V6 & 3.3 & $\mathrm{FF}$ & A & 19.9 & 7.1 & 9.2 \\
\hline T1 & FMC - FORD & RANGER 4X2 EV & $\mathrm{D}$ & $\mathrm{R}$ & E0 & 2.5 & EL & A & 29.7 & 5.2 & 1.8 \\
\hline T2 & FMC - FORD & F150 4X2 NGV & $\mathrm{D}$ & $\mathrm{R}$ & V8 & 5.4 & NG & A & 15.8 & 9.9 & 3.4 \\
\hline T4 & FMC - FORD & E250 VAN NGV & $\mathrm{D}$ & $\mathrm{R}$ & V8 & 5.4 & NG & A & 15.5 & 10.1 & 3.5 \\
\hline T1 & FMC - FORD & RANGER 4X2 FFV & $\mathrm{D}$ & $\mathrm{R}$ & V6 & 3.0 & FF & M & 19.7 & 7.1 & 9.2 \\
\hline T1 & FMC - FORD & RANGER 4X4 FFV & $\mathrm{D}$ & 4 & V6 & 3.0 & FF & M & 19.2 & 7.3 & 9.5 \\
\hline T1 & FMC - FORD & RANGER 4X2 FFV & $\mathrm{D}$ & $\mathrm{R}$ & V6 & 3.0 & $\mathrm{FF}$ & A & 18.5 & 7.6 & 9.7 \\
\hline T1 & GMC - CHEVROLET & S10 P/U 2WD & $\mathrm{D}$ & $\mathrm{R}$ & L4 & 2.2 & $\mathrm{FF}$ & M & 23.9 & 5.9 & 7.6 \\
\hline T1 & GMC - CHEVROLET & S10 P/U 2WD & $\mathrm{D}$ & $\mathrm{R}$ & L4 & 2.2 & $\mathrm{FF}$ & $A$ & 21.2 & 6.7 & 8.7 \\
\hline $\mathrm{T} 7$ & GMC - CHEVROLET & TRACKER CONV 2WD & $\mathrm{D}$ & $\mathrm{R}$ & L4 & 1.6 & G & M & 31.1 & 6.2 & 11.5 \\
\hline $\mathrm{T7}$ & GMC - CHEVROLET & TRACKER CONV 4X4 & $\mathrm{D}$ & 4 & L4 & 1.6 & G & M & 30.7 & 6.2 & 11.6 \\
\hline T1 & GMC & SONOMA 2WD & 1 & $\mathrm{R}$ & L4 & 2.2 & $\mathrm{FF}$ & M & 23.9 & 5.9 & 7.6 \\
\hline T1 & GMC & SONOMA 2WD & 1 & $\mathrm{R}$ & L4 & 2.2 & $\mathrm{FF}$ & A & 21.2 & 6.7 & 8.7 \\
\hline T1 & ISUZU & HOMBRE P/U 2WD & 1 & $\mathrm{R}$ & L4 & 2.2 & $\mathrm{FF}$ & M & 23.9 & 5.9 & 7.6 \\
\hline T1 & ISUZU & HOMBRE P/U 2WD & I & $\mathrm{R}$ & L4 & 2.2 & FF & A & 21.2 & 6.7 & 8.7 \\
\hline $\mathrm{T} 7$ & SUZUKI & VITARA CONV 2WD & 1 & $\mathrm{R}$ & L4 & 1.6 & G & M & 31.1 & 6.1 & 11.5 \\
\hline $\mathrm{T7}$ & SUZUKI & VITARA CONV 4WD & 1 & 4 & L4 & 1.6 & G & M & 30.7 & 6.2 & 11.6 \\
\hline T1 & TKM - MAZDA & MAZDA 4X2 FFV & 1 & $\mathrm{R}$ & V6 & 3.0 & $\mathrm{FF}$ & M & 19.7 & 7.1 & 9.2 \\
\hline T1 & TKM - MAZDA & MAZDA 4X4 FFV & 1 & 4 & V6 & 3.0 & $\mathrm{FF}$ & M & 19.2 & 7.3 & 9.5 \\
\hline T1 & TKM - MAZDA & MAZDA 4X2 FFV & 1 & $\mathrm{R}$ & V6 & 3.0 & $\mathrm{FF}$ & $A$ & 18.5 & 7.6 & 9.7 \\
\hline T7 & TOYOTA & RAV4 2WD & 1 & $\mathrm{~F}$ & L4 & 2.0 & G & M & 30.9 & 6.2 & 11.6 \\
\hline $\mathrm{T} 7$ & TOYOTA & RAV4 2WD & 1 & $F$ & L4 & 2.0 & G & $A$ & 30.5 & 6.3 & 11.7 \\
\hline
\end{tabular}


Energy Star Method 5 Vehicle List

Top 20\% of vehicles by footprint-MPG score

\begin{tabular}{|c|c|c|c|c|c|c|c|c|c|c|c|}
\hline $\begin{array}{l}\text { Type- } \\
\text { Size } \\
\text { Class }\end{array}$ & Manufacturer/Division & Name & $\begin{array}{c}\text { Dom I } \\
\text { Imp }\end{array}$ & DR & Cyl & Disp & Fuel & Trans & MPG & GHG & Oil Use \\
\hline \multicolumn{12}{|l|}{ Cars } \\
\hline$\overline{\mathrm{C} 4}$ & DAEWOO & LANOS- 3Dr & 1 & $F$ & L4 & 1.5 & G & $\mathrm{M}$ & 34.8 & 5.5 & 10.3 \\
\hline $\mathrm{C} 4$ & DAEWOO & LANOS-3Dr & 1 & $\mathrm{~F}$ & L4 & 1.6 & G & M & 34.6 & 5.5 & 10.3 \\
\hline $\mathrm{C} 4$ & DAEWOO & LANOS- 3Dr & 1 & $\mathrm{~F}$ & L4 & 1.5 & G & $A$ & 32.9 & 5.8 & 10.8 \\
\hline $\mathrm{C} 3$ & DCC - CHRYSLER & NEON & $\mathrm{D}$ & $\mathrm{F}$ & L4 & 2.0 & G & M & 36.1 & 5.3 & 9.9 \\
\hline $\mathrm{C} 4$ & DCC - CHRYSLER & CIRRUS & $\mathrm{D}$ & $\mathrm{F}$ & L4 & 2.0 & G & M & 34.3 & 5.6 & 10.4 \\
\hline C3 & DCC - CHRYSLER & NEON & $\mathrm{D}$ & $\mathrm{F}$ & L4 & 2.0 & G & A & 31.9 & 6.0 & 11.2 \\
\hline $\mathrm{C} 5$ & DCC - CHRYSLER & INTREPID & $\mathrm{D}$ & $\mathrm{F}$ & V6 & 2.7 & G & $A$ & 27.3 & 7.0 & 13.1 \\
\hline C5 & DCC - CHRYSLER & CONCORDE & $\mathrm{D}$ & $\mathrm{F}$ & V6 & 2.7 & G & $A$ & 27.3 & 7.0 & 13.1 \\
\hline C5 & DCC - CHRYSLER & CONCORDE & $\mathrm{D}$ & $\mathrm{F}$ & V6 & 3.2 & $\mathrm{G}$ & $A$ & 25.4 & 7.5 & 14.1 \\
\hline C5 & DCC - CHRYSLER & INTREPID & $\mathrm{D}$ & $\mathrm{F}$ & V6 & 3.2 & G & $A$ & 25.5 & 7.5 & 14.0 \\
\hline $\mathrm{C} 3$ & DCC - DODGE & NEON & $D$ & $\mathrm{~F}$ & L4 & 2.0 & G & M & 36.1 & 5.3 & 9.9 \\
\hline $\mathrm{C} 4$ & DCC - DODGE & STRATUS & $\mathrm{D}$ & $\mathrm{F}$ & L4 & 2.0 & G & M & 28.3 & 6.7 & 12.6 \\
\hline $\mathrm{C} 3$ & DCC - DODGE & NEON & $\mathrm{D}$ & $\mathrm{F}$ & L4 & 2.0 & G & $A$ & 31.9 & 6.0 & 11.2 \\
\hline $\mathrm{C} 4$ & DCC - DODGE & STRATUS & $\mathrm{D}$ & $\mathrm{F}$ & L4 & 2.0 & G & M & 28.3 & 6.7 & 12.6 \\
\hline C5 & DCC - DODGE & INTREPID & $\mathrm{D}$ & $\mathrm{F}$ & V6 & 2.7 & G & $A$ & 27.3 & 7.0 & 13.1 \\
\hline C5 & DCC - DODGE & INTREPID & $\mathrm{D}$ & $\mathrm{F}$ & V6 & 2.7 & G & A & 27.3 & 7.0 & 13.1 \\
\hline $\mathrm{C} 5$ & DCC - DODGE & INTREPID & $\mathrm{D}$ & $\mathrm{F}$ & V6 & 3.2 & G & $A$ & 25.5 & 7.5 & 14.0 \\
\hline $\mathrm{C} 4$ & DCC - MER-BENZ & E 320 & 1 & $\mathrm{R}$ & V6 & 3.2 & G & $A$ & 27.9 & 6.8 & 12.8 \\
\hline $\mathrm{C} 8$ & DCC - MER-BENZ & E 320 (WAGON) & 1 & $\mathrm{R}$ & V6 & 3.2 & G & $A$ & 26.8 & 7.1 & 13.3 \\
\hline $\mathrm{C} 4$ & DCC - MER-BENZ & E 320 4MATIC & 1 & 4 & V6 & 3.2 & G & $A$ & 26.9 & 7.1 & 13.3 \\
\hline $\mathrm{C} 3$ & DCC - PLYMOUTH & NEON & $\mathrm{D}$ & $\mathrm{F}$ & L4 & 2.0 & G & M & 36.1 & 5.3 & 9.9 \\
\hline $\mathrm{C} 4$ & DCC - PLYMOUTH & BREEZE & $\mathrm{D}$ & $\mathrm{F}$ & L4 & 2.0 & G & M & 35.4 & 5.4 & 10.1 \\
\hline C3 & DCC - PLYMOUTH & NEON & $\mathrm{D}$ & $\mathrm{F}$ & L4 & 2.0 & G & $A$ & 31.9 & 6.0 & 11.2 \\
\hline $\mathrm{C} 4$ & DCC - PLYMOUTH & BREEZE & $\mathrm{D}$ & $\mathrm{F}$ & L4 & 2.0 & G & $A$ & 28.3 & 6.7 & 12.6 \\
\hline $\mathrm{C} 7$ & FMC - FORD & FOCUS WAGON & $\mathrm{D}$ & $\mathrm{F}$ & L4 & 2.0 & G & M & 36.2 & 5.3 & 9.9 \\
\hline C3 & FMC - FORD & FOCUS & $\mathrm{D}$ & $\mathrm{F}$ & L4 & 2.0 & G & M & 35.7 & 5.4 & 10.0 \\
\hline C3 & FMC - FORD & ESCORT & $\mathrm{D}$ & $\mathrm{F}$ & L4 & 2.0 & G & M & 35.7 & 5.4 & 10.0 \\
\hline $\mathrm{C} 3$ & FMC - FORD & FOCUS & $\mathrm{D}$ & $\mathrm{F}$ & L4 & 2.0 & G & $A$ & 33.9 & 5.7 & 10.5 \\
\hline $\mathrm{C} 7$ & FMC - FORD & FOCUS WAGON & $\mathrm{D}$ & $\mathrm{F}$ & L4 & 2.0 & G & $A$ & 32.6 & 5.9 & 11.0 \\
\hline $\mathrm{C} 3$ & FMC - FORD & ESCORT & $\mathrm{D}$ & $\mathrm{F}$ & L4 & 2.0 & G & $A$ & 33.7 & 5.7 & 10.6 \\
\hline $\mathrm{C} 2$ & FMC - FORD & ESCORT ZX2 & $\mathrm{D}$ & $\mathrm{F}$ & L4 & 2.0 & G & M & 33.0 & 5.8 & 10.8 \\
\hline C3 & FMC - FORD & FOCUS 3D & $\mathrm{D}$ & $\mathrm{F}$ & L4 & 2.0 & G & M & 33.0 & 5.8 & 10.8 \\
\hline C3 & FMC - FORD & FOCUS 3D & $\mathrm{D}$ & $\mathrm{F}$ & L4 & 2.0 & G & $A$ & 32.4 & 5.9 & 11.0 \\
\hline $\mathrm{C} 2$ & FMC - FORD & ESCORT ZX2 & $\mathrm{D}$ & $\mathrm{F}$ & L4 & 2.0 & G & $A$ & 33.1 & 5.8 & 10.8 \\
\hline $\mathrm{C} 7$ & FMC - FORD & FOCUS WAGON & $\mathrm{D}$ & $\mathrm{F}$ & L4 & 2.0 & G & A & 32.6 & 5.9 & 11.0 \\
\hline $\mathrm{C} 3$ & FMC - FORD & CONTOUR & $\mathrm{D}$ & $F$ & L4 & 2.0 & G & $\mathrm{M}$ & 32.6 & 5.9 & 11.0 \\
\hline C3 & FMC - FORD & CONTOUR & $\mathrm{D}$ & $\mathrm{F}$ & L4 & 2.0 & G & A & 30.3 & 6.3 & 11.8 \\
\hline $\mathrm{C} 3$ & FMC - FORD & CONTOUR & $\mathrm{D}$ & $\mathrm{F}$ & L4 & 2.0 & G & $A$ & 30.3 & 6.3 & 11.8 \\
\hline C5 & FMC - LINCOLN & TOWN CAR & $\mathrm{D}$ & $\mathrm{R}$ & V8 & 4.6 & G & $A$ & 23.3 & 8.1 & 15.3 \\
\hline C3 & FMC - MAZDA & PROTEGE & 1 & $F$ & L4 & 1.6 & G & A & 33.8 & 5.7 & 10.6 \\
\hline $\mathrm{C} 4$ & FMC - MAZDA & 626 & I & $\mathrm{F}$ & L4 & 2.0 & G & M & 33.5 & 5.7 & 10.6 \\
\hline C3 & FMC - MAZDA & PROTEGE & 1 & $\mathrm{~F}$ & L4 & 1.8 & G & M & 32.4 & 5.9 & 11.0 \\
\hline C3 & FMC - MAZDA & PROTEGE & 1 & $\mathrm{~F}$ & L4 & 1.6 & G & $\mathrm{M}$ & 36.5 & 5.3 & 9.8 \\
\hline
\end{tabular}




\begin{tabular}{|c|c|c|c|c|c|c|c|c|c|c|c|}
\hline $\begin{array}{l}\text { Type- } \\
\text { Size } \\
\text { Class }\end{array}$ & Manufacturer/Division & Name & $\begin{array}{c}\text { Dom I } \\
\text { Imp }\end{array}$ & DR & Cyl & Disp & Fuel & Trans & MPG & GHG & Oil Use \\
\hline$\overline{C 3}$ & FMC - MERCURY & MYSTIQUE & $\bar{D}$ & $\mathrm{~F}$ & $\mathrm{~L} 4$ & 2.0 & $\mathrm{G}$ & $\mathrm{M}$ & 32.4 & 5.9 & 11.0 \\
\hline C3 & FMC - MERCURY & COUGAR & $\mathrm{D}$ & $\mathrm{F}$ & L4 & 2.0 & G & $\mathrm{M}$ & 32.4 & 5.9 & 11.0 \\
\hline C3 & FMC - MERCURY & MYSTIQUE & $\mathrm{D}$ & $\mathrm{F}$ & L4 & 2.0 & G & A & 29.6 & 6.5 & 12.1 \\
\hline C4 & GMC - BUICK & CENTURY & $\mathrm{D}$ & $\mathrm{F}$ & V6 & 3.1 & G & A & 27.5 & 6.9 & 13.0 \\
\hline C5 & GMC - BUICK & LESABRE & $\mathrm{D}$ & $\mathrm{F}$ & V6 & 3.8 & G & A & 26.6 & 7.2 & 13.5 \\
\hline C5 & GMC - BUICK & PARK AVENUE & $\mathrm{D}$ & $\mathrm{F}$ & V6 & 3.8 & G & A & 26.6 & 7.2 & 13.5 \\
\hline C2 & GMC - CHEVY & METRO & I & $\mathrm{F}$ & L3 & 1.0 & G & M & 48.9 & 4.0 & 7.3 \\
\hline C2 & GMC - CHEVY & METRO & I & $\mathrm{F}$ & L4 & 1.3 & G & M & 45.5 & 4.2 & 7.8 \\
\hline C3 & GMC - CHEVY & PRIZM & I & $\mathrm{F}$ & $\mathrm{L} 4$ & 1.8 & G & M & 39.6 & 4.9 & 9.0 \\
\hline C3 & GMC - CHEVY & PRIZM & I & $\mathrm{F}$ & L4 & 1.8 & G & A & 37.5 & 5.1 & 9.5 \\
\hline C2 & GMC - CHEVY & METRO & I & $\mathrm{F}$ & L4 & 1.3 & G & A & 37.6 & 5.1 & 9.5 \\
\hline C3 & GMC - CHEVY & PRIZM & I & $\mathrm{F}$ & L4 & 1.8 & G & A & 34.9 & 5.5 & 10.2 \\
\hline C2 & GMC - CHEVY & CAVALIER & $\mathrm{D}$ & $\mathrm{F}$ & L4 & 2.2 & G & M & 32.1 & 6.0 & 11.1 \\
\hline C2 & GMC - CHEVY & CAVALIER & $\mathrm{D}$ & $\mathrm{F}$ & L4 & 2.2 & G & A & 30.7 & 6.2 & 11.6 \\
\hline C2 & GMC - CHEVY & CAVALIER Z24 & $\mathrm{D}$ & $\mathrm{F}$ & L4 & 2.4 & G & M & 30.8 & 6.2 & 11.6 \\
\hline C4 & GMC - CHEVY & MONTECARLO & $\mathrm{D}$ & $\mathrm{F}$ & V6 & 3.4 & G & A & 27.5 & 6.9 & 13.0 \\
\hline C4 & GMC - CHEVY & LUMINA & D & $\mathrm{F}$ & V6 & 3.1 & G & A & 27.5 & 6.9 & 13.0 \\
\hline C4 & GMC - CHEVY & MALIBU & $\mathrm{D}$ & $\mathrm{F}$ & V6 & 3.1 & G & A & 27.5 & 6.9 & 13.0 \\
\hline C5 & GMC - CHEVY & IMPALA & $\mathrm{D}$ & $\mathrm{F}$ & V6 & 3.4 & G & A & 27.5 & 6.9 & 13.0 \\
\hline C5 & GMC - CHEVY & IMPALA & $\mathrm{D}$ & $\mathrm{F}$ & V6 & 3.8 & G & A & 26.9 & 7.1 & 13.3 \\
\hline C3 & GMC - OLDSMOBILE & ALERO & $\mathrm{D}$ & $\mathrm{F}$ & L4 & 2.4 & G & M & 29.5 & 6.5 & 12.1 \\
\hline C3 & GMC - OLDSMOBILE & ALERO & $\mathrm{D}$ & $\mathrm{F}$ & L4 & 2.4 & G & A & 29.4 & 6.5 & 12.1 \\
\hline C2 & GMC - PONTIAC & SUNFIRE & $\mathrm{D}$ & $\mathrm{F}$ & L4 & 2.2 & G & M & 32.1 & 6.0 & 11.1 \\
\hline C2 & GMC - PONTIAC & SUNFIRE CNVT & $\mathrm{D}$ & $\mathrm{F}$ & L4 & 2.2 & G & A & 30.7 & 6.2 & 11.6 \\
\hline C2 & GMC - PONTIAC & SUNFIRE & $\mathrm{D}$ & $\mathrm{F}$ & L4 & 2.4 & G & M & 30.8 & 6.2 & 11.6 \\
\hline C3 & GMC - PONTIAC & GRAND AM & $\mathrm{D}$ & $\mathrm{F}$ & L4 & 2.4 & G & M & 29.5 & 6.5 & 12.1 \\
\hline C3 & GMC - PONTIAC & GRAND AM & D & $\mathrm{F}$ & L4 & 2.4 & G & A & 29.4 & 6.5 & 12.1 \\
\hline C4 & GMC - PONTIAC & GRAND PRIX 4Dr & $\mathrm{D}$ & $\mathrm{F}$ & V6 & 3.1 & G & A & 27.5 & 6.9 & 13.0 \\
\hline C4 & GMC - PONTIAC & GRAND PRIX & $\mathrm{D}$ & $\mathrm{F}$ & V6 & 3.8 & G & A & 26.9 & 7.1 & 13.3 \\
\hline C5 & GMC - PONTIAC & BONNEVILLE & $\mathrm{D}$ & $\mathrm{F}$ & V6 & 3.8 & G & A & 26.6 & 7.2 & 13.5 \\
\hline C3 & GMC - SATURN & SL & $\mathrm{D}$ & $\mathrm{F}$ & L4 & 1.9 & G & $\mathrm{M}$ & 38.7 & 5.0 & 9.2 \\
\hline C2 & GMC - SATURN & SC & $\mathrm{D}$ & $\mathrm{F}$ & L4 & 1.9 & G & M & 35.4 & 5.4 & 10.1 \\
\hline C2 & GMC - SATURN & SC & $\mathrm{D}$ & $\mathrm{F}$ & L4 & 1.9 & G & M & 38.1 & 5.0 & 9.4 \\
\hline C3 & GMC - SATURN & SL & $\mathrm{D}$ & $\mathrm{F}$ & L4 & 1.9 & G & M & 36.1 & 5.3 & 9.9 \\
\hline C7 & GMC - SATURN & SW & D & $\mathrm{F}$ & L4 & 1.9 & G & M & 36.1 & 5.3 & 9.9 \\
\hline C3 & GMC - SATURN & SL & $\mathrm{D}$ & $\mathrm{F}$ & L4 & 1.9 & G & A & 34.4 & 5.6 & 10.4 \\
\hline $\mathrm{C} 2$ & GMC - SATURN & SC & $\mathrm{D}$ & $\mathrm{F}$ & L4 & 1.9 & G & A & 33.9 & 5.7 & 10.5 \\
\hline C7 & GMC - SATURN & SW & $\mathrm{D}$ & $\mathrm{F}$ & $\mathrm{L} 4$ & 1.9 & G & A & 35.4 & 5.4 & 10.1 \\
\hline C2 & GMC - SATURN & SC & $\mathrm{D}$ & $\mathrm{F}$ & L4 & 1.9 & G & A & 36.1 & 5.3 & 9.9 \\
\hline C3 & GMC - SATURN & SL & $\mathrm{D}$ & $\mathrm{F}$ & L4 & 1.9 & G & A & 36.0 & 5.3 & 9.9 \\
\hline C7 & GMC - SATURN & SW & $\mathrm{D}$ & $\mathrm{F}$ & L4 & 1.9 & G & A & 33.9 & 5.7 & 10.5 \\
\hline C4 & GMC - SATURN & LS & D & $\mathrm{F}$ & L4 & 2.2 & G & M & 31.9 & 6.0 & 11.2 \\
\hline C4 & GMC - SATURN & LS & $\mathrm{D}$ & $\mathrm{F}$ & L4 & 2.2 & G & A & 30.5 & 6.3 & 11.7 \\
\hline C8 & GMC - SATURN & LW & $\mathrm{D}$ & $\mathrm{F}$ & L4 & 2.2 & G & A & 29.4 & 6.5 & 12.1 \\
\hline C8 & GMC - SATURN & LW & $\mathrm{D}$ & $\mathrm{F}$ & V6 & 3.0 & G & A & 26.2 & 7.3 & 13.6 \\
\hline C2 & HONDA - ACURA & ACURA INTEGRA & 1 & $\mathrm{~F}$ & L4 & 1.8 & G & M & 32.3 & 5.9 & 11.0 \\
\hline C2 & HONDA - ACURA & ACURA INTEGRA -R & I & $\mathrm{F}$ & L4 & 1.8 & G & $\mathrm{M}$ & 31.9 & 6.0 & 11.2 \\
\hline
\end{tabular}




\begin{tabular}{|c|c|c|c|c|c|c|c|c|c|c|c|}
\hline $\begin{array}{l}\text { Type- } \\
\text { Size } \\
\text { Class }\end{array}$ & Manufacturer/Division & Name & $\begin{array}{c}\text { Dom I } \\
\text { Imp }\end{array}$ & DR & Cyl & Disp & Fuel & Trans & MPG & GHG & Oil Use \\
\hline$\overline{\mathrm{C} 2}$ & HONDA - ACURA & ACURA INTEGRA & $\mathrm{T}$ & $\mathrm{F}$ & $\mathrm{L} 4$ & 1.8 & $\mathrm{G}$ & $\bar{A}$ & 30.7 & 6.2 & 11.6 \\
\hline C6 & HONDA & INSIGHT & I & $\mathrm{F}$ & L3 & 1.0 & G/EL & $\mathrm{M}$ & 76.3 & 2.6 & 4.7 \\
\hline $\mathrm{C} 2$ & HONDA & CIVIC HX & I & $\mathrm{F}$ & L4 & 1.6 & G & M & 44.7 & 4.3 & 8.0 \\
\hline C2 & HONDA & CIVIC HX & I & $\mathrm{F}$ & $\mathrm{L} 4$ & 1.6 & G & A & 41.9 & 4.6 & 8.5 \\
\hline C2 & HONDA & CIVIC dx 4Dr & I & $\mathrm{F}$ & L4 & 1.6 & G & M & 37.2 & 5.2 & 9.6 \\
\hline C2 & HONDA & CIVIC ex & I & $\mathrm{F}$ & L4 & 1.6 & G & $\mathrm{M}$ & 37.2 & 5.2 & 9.6 \\
\hline C2 & HONDA & CIVIC vp & I & $\mathrm{F}$ & L4 & 1.6 & G & A & 35.5 & 5.4 & 10.1 \\
\hline C2 & HONDA & CIVIC dx 4Dr & I & $\mathrm{F}$ & L4 & 1.6 & G & A & 35.6 & 5.4 & 10.0 \\
\hline $\mathrm{C} 2$ & HONDA & CIVIC ex & I & $\mathrm{F}$ & $\mathrm{L} 4$ & 1.6 & G & A & 35.5 & 5.4 & 10.1 \\
\hline C2 & HONDA & CIVIC si & I & $\mathrm{F}$ & $\mathrm{L} 4$ & 1.6 & G & M & 32.8 & 5.8 & 10.9 \\
\hline C4 & HONDA & ACCORD Ix & I & $\mathrm{F}$ & L4 & 2.3 & G & M & 32.1 & 6.0 & 11.1 \\
\hline C4 & HONDA & ACCORD & I & $\mathrm{F}$ & L4 & 2.3 & G & $\mathrm{M}$ & 30.4 & 6.3 & 11.8 \\
\hline C4 & HONDA & ACCORD Ix & I & $\mathrm{F}$ & L4 & 2.3 & G & A & 29.9 & 6.4 & 11.9 \\
\hline C4 & HONDA & ACCORD & I & $\mathrm{F}$ & L4 & 2.3 & G & A & 28.6 & 6.7 & 12.5 \\
\hline C3 & HYUNDAI & ACCENT & I & $\mathrm{F}$ & L4 & 1.5 & G & M & 36.6 & 5.3 & 9.8 \\
\hline C3 & HYUNDAI & ELANTRA & I & $\mathrm{F}$ & L4 & 2.0 & G & $\mathrm{M}$ & 32.1 & 6.0 & 11.1 \\
\hline C2 & MITSUBISHI & MIRAGE 2D & I & $\mathrm{F}$ & $\mathrm{L} 4$ & 1.5 & G & M & 41.7 & 4.6 & 8.6 \\
\hline C2 & MITSUBISHI & MIRAGE & I & $\mathrm{F}$ & L4 & 1.8 & G & M & 36.3 & 5.3 & 9.8 \\
\hline C2 & MITSUBISHI & MIRAGE 2D & I & $\mathrm{F}$ & L4 & 1.5 & G & A & 36.7 & 5.2 & 9.7 \\
\hline C2 & MITSUBISHI & MIRAGE & I & $\mathrm{F}$ & $\mathrm{L} 4$ & 1.8 & G & A & 33.2 & 5.8 & 10.8 \\
\hline C3 & NISSAN & SENTRA & I & $\mathrm{F}$ & $\mathrm{L} 4$ & 1.8 & G & M & 34.9 & 5.5 & 10.2 \\
\hline C3 & NISSAN & SENTRA & I & $\mathrm{F}$ & $\mathrm{L} 4$ & 1.8 & G & A & 33.9 & 5.6 & 10.5 \\
\hline C3 & NISSAN & ALTIMA & I & $\mathrm{F}$ & L4 & 2.4 & G & M & 30.9 & 6.2 & 11.6 \\
\hline C4 & NISSAN & MAXIMA & I & $\mathrm{F}$ & L6 & 3.0 & G & M & 27.8 & 6.9 & 12.8 \\
\hline C2 & SUZUKI & SWIFT & I & $\mathrm{F}$ & $\mathrm{L} 4$ & 1.3 & G & $\mathrm{M}$ & 45.5 & 4.2 & 7.8 \\
\hline C2 & SUZUKI & ESTEEM & I & $\mathrm{F}$ & $\mathrm{L} 4$ & 1.6 & G & $\mathrm{M}$ & 38.6 & 5.0 & 9.3 \\
\hline C2 & SUZUKI & SWIFT & I & $\mathrm{F}$ & $\mathrm{L} 4$ & 1.3 & G & A & 37.6 & 5.1 & 9.5 \\
\hline C2 & SUZUKI & ESTEEM & I & $\mathrm{F}$ & L4 & 1.8 & G & M & 36.2 & 5.3 & 9.9 \\
\hline $\mathrm{C} 7$ & SUZUKI & ESTEEM WAGON & I & $\mathrm{F}$ & L4 & 1.8 & G & M & 35.1 & 5.5 & 10.2 \\
\hline C2 & SUZUKI & ESTEEM & I & $\mathrm{F}$ & $\mathrm{L} 4$ & 1.6 & G & A & 35.0 & 5.5 & 10.2 \\
\hline $\mathrm{C7}$ & SUZUKI & ESTEEM WAGON & I & $\mathrm{F}$ & $\mathrm{L} 4$ & 1.6 & G & A & 33.7 & 5.7 & 10.6 \\
\hline $\mathrm{C} 7$ & SUZUKI & ESTEEM WAGON & I & $\mathrm{F}$ & L4 & 1.8 & G & A & 33.7 & 5.7 & 10.6 \\
\hline C2 & SUZUKI & ESTEEM & I & $\mathrm{F}$ & L4 & 1.8 & G & A & 33.7 & 5.7 & 10.6 \\
\hline C4 & TOYOTA & CAMRY CNG & I & $\mathrm{F}$ & L4 & 2.2 & NG & A & 30.2 & 5.4 & 1.8 \\
\hline C3 & TOYOTA & $\mathrm{ECHO}$ & I & $\mathrm{F}$ & L4 & 1.5 & G & M & 43.1 & 4.5 & 8.3 \\
\hline C3 & TOYOTA & COROLLA & I & $\mathrm{F}$ & L4 & 1.8 & G & $M$ & 39.6 & 4.9 & 9.0 \\
\hline C3 & TOYOTA & $\mathrm{ECHO}$ & I & $\mathrm{F}$ & L4 & 1.5 & G & A & 39.4 & 4.9 & 9.1 \\
\hline C3 & TOYOTA & COROLLA & I & $\mathrm{F}$ & L4 & 1.8 & G & A & 37.4 & 5.1 & 9.6 \\
\hline C2 & TOYOTA & CELICA & I & $\mathrm{F}$ & L4 & 1.8 & G & M & 35.2 & 5.4 & 10.1 \\
\hline C2 & TOYOTA & CELICA & I & $\mathrm{F}$ & L4 & 1.8 & G & $A$ & 34.9 & 5.5 & 10.2 \\
\hline C3 & TOYOTA & COROLLA & I & $\mathrm{F}$ & L4 & 1.8 & G & A & 34.9 & 5.5 & 10.2 \\
\hline C3 & TOYOTA & CAMRY SOLARA & I & $\mathrm{F}$ & L4 & 2.2 & G & M & 31.0 & 6.2 & 11.5 \\
\hline C4 & TOYOTA & CAMRY & I & $\mathrm{F}$ & L4 & 2.2 & G & M & 31.0 & 6.2 & 11.5 \\
\hline C2 & TOYOTA & CELICA & I & $\mathrm{F}$ & L4 & 1.8 & G & M & 30.8 & 6.2 & 11.6 \\
\hline C3 & TOYOTA & CAMRY SOLARA & I & $\mathrm{F}$ & L4 & 2.2 & G & A & 30.1 & 6.4 & 11.9 \\
\hline C4 & TOYOTA & CAMRY & I & $\mathrm{F}$ & L4 & 2.2 & G & A & 30.1 & 6.4 & 11.9 \\
\hline C2 & TOYOTA & CAMRY SOLARA & I & $\mathrm{F}$ & L4 & 2.2 & G & A & 30.1 & 6.4 & 11.9 \\
\hline
\end{tabular}




\begin{tabular}{|c|c|c|c|c|c|c|c|c|c|c|c|}
\hline $\begin{array}{l}\text { Type- } \\
\text { Size } \\
\text { Class }\end{array}$ & Manufacturer/Division & Name & $\begin{array}{c}\text { Dom I } \\
\text { Imp }\end{array}$ & DR & Cyl & Disp & Fuel & Trans & MPG & GHG & Oil Use \\
\hline & & CONVERT & & & & & & & & & \\
\hline $\mathrm{C} 5$ & TOYOTA & AVALON & 1 & $F$ & V6 & 3.0 & G & $A$ & 28.1 & 6.8 & 12.7 \\
\hline C3 & VWA - AUDI & AUDI A4 & I & $\mathrm{F}$ & L4 & 1.8 & G & M & 31.9 & 6.0 & 11.2 \\
\hline C2 & VWA & NEW BEETLE & 1 & $F$ & L4 & 1.9 & $\mathrm{D}$ & M & 52.7 & 4.0 & 6.8 \\
\hline C3 & VWA & GOLF TDI & 1 & $\mathrm{~F}$ & L4 & 1.9 & $\mathrm{D}$ & $M$ & 52.7 & 4.0 & 6.8 \\
\hline C3 & VWA & JETTA & I & $\mathrm{F}$ & L4 & 1.9 & D & $\mathrm{M}$ & 52.7 & 4.0 & 6.8 \\
\hline C2 & VWA & NEW BEETLE & I & $\mathrm{F}$ & L4 & 1.9 & D & A & 44.7 & 4.7 & 8.0 \\
\hline C3 & VWA & GOLF TDI & 1 & $\mathrm{~F}$ & L4 & 1.9 & $\mathrm{D}$ & A & 44.7 & 4.7 & 8.0 \\
\hline C3 & VWA & JETTA & 1 & $F$ & L4 & 1.9 & $\mathrm{D}$ & $A$ & 44.7 & 4.7 & 8.0 \\
\hline $\mathrm{C} 2$ & VWA & NEW BEETLE & I & $\mathrm{F}$ & L4 & 1.8 & G & M & 32.3 & 5.9 & 11.1 \\
\hline C3 & VWA & GTI & 1 & $\mathrm{~F}$ & L4 & 1.8 & G & M & 31.3 & 6.1 & 11.4 \\
\hline C3 & VWA & GOLF & 1 & $\mathrm{~F}$ & L4 & 1.8 & G & M & 31.3 & 6.1 & 11.4 \\
\hline C3 & VWA & JETTA & I & $\mathrm{F}$ & L4 & 1.8 & G & M & 31.3 & 6.1 & 11.4 \\
\hline C4 & VWA & PASSAT & 1 & $\mathrm{~F}$ & L4 & 1.8 & G & M & 31.3 & 6.1 & 11.4 \\
\hline C8 & VWA & PASSAT WAGON & I & $\mathrm{F}$ & L4 & 1.8 & G & M & 31.3 & 6.1 & 11.4 \\
\hline \multicolumn{12}{|c|}{ Trucks } \\
\hline T3 & DCC - CHRYSLER & $\begin{array}{l}\text { TOWN \& COUNTRY } \\
\text { 2WD }\end{array}$ & $\mathrm{D}$ & $\mathrm{F}$ & V6 & 3.3 & G & $A$ & 23.2 & 8.2 & 15.4 \\
\hline T3 & DCC - DODGE & CARAVAN 2WD & $\mathrm{D}$ & $\mathrm{F}$ & L4 & 2.4 & G & $A$ & 26.0 & 7.3 & 13.7 \\
\hline T3 & DCC - DODGE & CARAVAN 2WD & D & $\mathrm{F}$ & V6 & 3.3 & G & A & 24.3 & 7.8 & 14.7 \\
\hline T3 & DCC - PLYMOUTH & $\begin{array}{l}\text { CARAVAN/VOYAGER } \\
\text { 2WD }\end{array}$ & D & $\mathrm{F}$ & L4 & 2.4 & G & $A$ & 25.9 & 7.3 & 13.8 \\
\hline $\mathrm{T} 1$ & FMC - FORD & RANGER 4X2 EV & $\mathrm{D}$ & $\mathrm{R}$ & E0 & 2.5 & EL & $A$ & 29.7 & 5.2 & 1.8 \\
\hline T1 & FMC - FORD & RANGER 4X2 & $\mathrm{D}$ & $\mathrm{R}$ & L4 & 2.5 & G & M & 28.3 & 6.7 & 12.6 \\
\hline T3 & FMC - FORD & WINDSTAR VAN & $\mathrm{D}$ & $\mathrm{F}$ & V6 & 3.8 & G & $A$ & 23.0 & 8.2 & 15.5 \\
\hline T4 & FMC - FORD & E150 VAN & $\mathrm{D}$ & $\mathrm{R}$ & V8 & 4.6 & G & A & 19.5 & 9.7 & 18.4 \\
\hline T1 & GMC - CHEVROLET & S10 P/U 2WD & $\mathrm{D}$ & $\mathrm{R}$ & L4 & 2.2 & G & M & 29.6 & 6.5 & 12.1 \\
\hline T1 & GMC & SONOMA 2WD & 1 & $\mathrm{R}$ & L4 & 2.2 & G & M & 29.6 & 6.5 & 12.1 \\
\hline $\mathrm{T} 2$ & GMC & C1500 SIERRA 2WD & 1 & $\mathrm{R}$ & V6 & 4.3 & G & M & 22.8 & 8.3 & 15.7 \\
\hline T3 & GMC - OLDSMOBILE & SILHOUETTE 2WD & $\mathrm{D}$ & $\mathrm{F}$ & V6 & 3.4 & G & A & 25.0 & 7.6 & 14.3 \\
\hline T3 & HONDA & ODYSSEY & 1 & $\mathrm{~F}$ & V6 & 3.5 & G & A & 24.0 & 7.9 & 14.9 \\
\hline T5 & SUBARU & FORESTER & 1 & 4 & $\mathrm{HO} 4$ & 2.5 & G & M & 27.9 & 6.8 & 12.8 \\
\hline T5 & SUBARU & FORESTER & 1 & 4 & $\mathrm{HO} 4$ & 2.5 & G & $A$ & 27.9 & 6.8 & 12.8 \\
\hline T1 & TKM - MAZDA & MAZDA 4X2 & 1 & $\mathrm{R}$ & L4 & 2.5 & G & M & 28.3 & 6.7 & 12.6 \\
\hline
\end{tabular}


Energy Star Method 6 Vehicle List

Top $20 \%$ of vehicles by Ratio of Actual to Predicted MPG (equation 1)

\begin{tabular}{|c|c|c|c|c|c|c|c|c|c|c|c|}
\hline $\begin{array}{l}\text { Type-Size } \\
\text { Class }\end{array}$ & Manufacturer/Division & Name & $\begin{array}{l}\text { Dom I } \\
\text { Imp }\end{array}$ & DR & Cyl & Disp & Fuel & Trans & MPG & GHG & Oil Use \\
\hline \multicolumn{12}{|l|}{ Cars } \\
\hline$\overline{\mathrm{C} 2}$ & BMW & $328 \mathrm{i}, \mathrm{Ci}$ & I & $\mathrm{R}$ & L6 & 2.8 & $\mathrm{G}$ & $M$ & 27.6 & 6.9 & 12.9 \\
\hline C3 & BMW & $528 \mathrm{i}$ & 1 & $\mathrm{R}$ & L6 & 2.8 & G & M & 27.6 & 6.9 & 12.9 \\
\hline C3 & BMW & $540 \mathrm{iA}$ & 1 & $\mathrm{R}$ & V8 & 4.4 & $\mathrm{G}$ & A & 23.7 & 8.0 & 15.1 \\
\hline C4 & BMW & $740 \mathrm{iA}$ & I & $\mathrm{R}$ & V8 & 4.4 & G & $A$ & 22.7 & 8.4 & 15.8 \\
\hline C5 & BMW & 740iLA & 1 & $\mathrm{R}$ & V8 & 4.4 & G & $A$ & 22.7 & 8.4 & 15.8 \\
\hline C3 & DCC - CHRYSLER & NEON & $\mathrm{D}$ & $\mathrm{F}$ & L4 & 2.0 & G & M & 36.1 & 5.3 & 9.9 \\
\hline C4 & DCC - CHRYSLER & CIRRUS & $\mathrm{D}$ & $\mathrm{F}$ & L4 & 2.0 & $\mathrm{G}$ & $M$ & 34.3 & 5.6 & 10.4 \\
\hline C3 & DCC - CHRYSLER & NEON & $\mathrm{D}$ & $\mathrm{F}$ & L4 & 2.0 & $\mathrm{G}$ & $A$ & 31.9 & 6.0 & 11.2 \\
\hline C5 & DCC - CHRYSLER & INTREPID & $\mathrm{D}$ & $\mathrm{F}$ & V6 & 2.7 & G & $A$ & 27.3 & 7.0 & 13.1 \\
\hline C5 & DCC - CHRYSLER & CONCORDE & $\mathrm{D}$ & $\mathrm{F}$ & V6 & 2.7 & G & A & 27.3 & 7.0 & 13.1 \\
\hline C5 & DCC - CHRYSLER & CONCORDE & $\mathrm{D}$ & $\mathrm{F}$ & V6 & 3.2 & G & A & 25.4 & 7.5 & 14.1 \\
\hline C5 & DCC - CHRYSLER & INTREPID & $\mathrm{D}$ & $\mathrm{F}$ & V6 & 3.2 & G & $A$ & 25.5 & 7.5 & 14.0 \\
\hline C5 & DCC - CHRYSLER & $300 \mathrm{M}$ & $\mathrm{D}$ & $\mathrm{F}$ & V6 & 3.5 & $G$ & $A$ & 24.8 & 7.7 & 14.4 \\
\hline C5 & DCC - CHRYSLER & LHS & $\mathrm{D}$ & $\mathrm{F}$ & V6 & 3.5 & $\mathrm{G}$ & $A$ & 24.4 & 7.8 & 14.6 \\
\hline C3 & DCC - DODGE & NEON & $\mathrm{D}$ & $\mathrm{F}$ & L4 & 2.0 & G & M & 36.1 & 5.3 & 9.9 \\
\hline C3 & DCC - DODGE & NEON & $\mathrm{D}$ & $\mathrm{F}$ & L4 & 2.0 & G & A & 31.9 & 6.0 & 11.2 \\
\hline C5 & DCC - DODGE & INTREPID & $\mathrm{D}$ & $\mathrm{F}$ & V6 & 2.7 & G & A & 27.3 & 7.0 & 13.1 \\
\hline C5 & DCC - DODGE & INTREPID & $\mathrm{D}$ & $\mathrm{F}$ & V6 & 2.7 & $\mathrm{G}$ & $A$ & 27.3 & 7.0 & 13.1 \\
\hline C5 & DCC - DODGE & INTREPID & $\mathrm{D}$ & $\mathrm{F}$ & V6 & 3.2 & G & $A$ & 25.5 & 7.5 & 14.0 \\
\hline C5 & DCC - DODGE & INTREPID & $\mathrm{D}$ & $\mathrm{F}$ & V6 & 3.5 & G & A & 24.8 & 7.7 & 14.4 \\
\hline $\mathrm{C} 6$ & DCC - MER-BENZ & $\begin{array}{l}\text { SLK } 230 \\
\text { KOMPRESSOR }\end{array}$ & I & $\mathrm{R}$ & L4 & 2.3 & G & M & 28.7 & 6.7 & 12.5 \\
\hline C3 & DCC - MER-BENZ & $\begin{array}{l}\text { C } 230 \\
\text { KOMPRESSOR }\end{array}$ & 1 & $\mathrm{R}$ & L4 & 2.3 & G & A & 28.3 & 6.7 & 12.6 \\
\hline $\mathrm{C} 6$ & DCC - MER-BENZ & $\begin{array}{l}\text { SLK } 230 \\
\text { KOMPRESSOR }\end{array}$ & 1 & $\mathrm{R}$ & L4 & 2.3 & G & A & 29.2 & 6.5 & 12.2 \\
\hline C1 & DCC - MER-BENZ & $\begin{array}{l}\text { CLK } 320 \\
\text { (CABRIOLET) }\end{array}$ & 1 & $\mathrm{R}$ & V6 & 3.2 & G & A & 26.5 & 7.2 & 13.5 \\
\hline C4 & DCC - MER-BENZ & E 320 & I & $\mathrm{R}$ & V6 & 3.2 & G & A & 27.9 & 6.8 & 12.8 \\
\hline $\mathrm{C} 8$ & DCC - MER-BENZ & E 320 (WAGON) & I & $\mathrm{R}$ & V6 & 3.2 & $\mathrm{G}$ & $A$ & 26.8 & 7.1 & 13.3 \\
\hline C2 & DCC - MER-BENZ & CLK 320 & 1 & $\mathrm{R}$ & V6 & 3.2 & G & A & 27.5 & 6.9 & 13.0 \\
\hline C3 & DCC - MER-BENZ & C 280 & I & $\mathrm{R}$ & V6 & 2.8 & G & $A$ & 27.3 & 7.0 & 13.1 \\
\hline $\mathrm{C} 4$ & DCC - MER-BENZ & E 320 4MATIC & I & 4 & V6 & 3.2 & G & $A$ & 26.9 & 7.1 & 13.3 \\
\hline C8 & DCC - MER-BENZ & $\begin{array}{l}\text { E } 320 \\
\text { 4MATIC(WAGON) }\end{array}$ & I & 4 & V6 & 3.2 & $\mathrm{G}$ & A & 25.9 & 7.3 & 13.8 \\
\hline $\mathrm{C} 2$ & DCC - MER-BENZ & CLK 430 & I & $\mathrm{R}$ & V8 & 4.3 & G & A & 24.2 & 7.8 & 14.7 \\
\hline C3 & DCC - MER-BENZ & C 43 AMG & 1 & $\mathrm{R}$ & V8 & 4.3 & G & $A$ & 23.1 & 8.2 & 15.4 \\
\hline $\mathrm{C} 1$ & DCC - MER-BENZ & $\begin{array}{l}\text { CLK } 430 \\
\text { (CABRIOLET) }\end{array}$ & 1 & $\mathrm{R}$ & V8 & 4.3 & G & $A$ & 23.1 & 8.2 & 15.5 \\
\hline C4 & DCC - MER-BENZ & E 430 & 1 & $\mathrm{R}$ & V8 & 4.3 & G & A & 23.9 & 7.9 & 14.9 \\
\hline C5 & DCC - MER-BENZ & S 430 & I & $\mathrm{R}$ & V8 & 4.3 & G & $A$ & 23.0 & 8.2 & 15.5 \\
\hline $\mathrm{C} 4$ & DCC - MER-BENZ & E 430 4MATIC & I & 4 & V8 & 4.3 & $\mathrm{G}$ & $A$ & 22.8 & 8.3 & 15.7 \\
\hline $\mathrm{C} 4$ & DCC - MER-BENZ & E 55 AMG & I & $\mathrm{R}$ & V8 & 5.5 & G & $A$ & 22.0 & 8.6 & 16.2 \\
\hline C3 & DCC - MER-BENZ & CL 500 & 1 & $\mathrm{R}$ & V8 & 5.0 & G & A & 21.7 & 8.7 & 16.4 \\
\hline C5 & DCC - MER-BENZ & S 500 & 1 & $\mathrm{R}$ & V8 & 5.0 & G & A & 21.7 & 8.7 & 16.4 \\
\hline $\mathrm{C} 6$ & DCC - MER-BENZ & SL 500 & 1 & $\mathrm{R}$ & V8 & 5.0 & G & A & 21.6 & 8.8 & 16.5 \\
\hline C3 & DCC - PLYMOUTH & NEON & $\mathrm{D}$ & $\mathrm{F}$ & L4 & 2.0 & G & M & 36.1 & 5.3 & 9.9 \\
\hline
\end{tabular}




\begin{tabular}{|c|c|c|c|c|c|c|c|c|c|c|c|}
\hline $\begin{array}{l}\text { Type-Size } \\
\text { Class }\end{array}$ & Manufacturer/Division & Name & $\begin{array}{l}\text { Dom I } \\
\text { Imp }\end{array}$ & DR & Cyl & Disp & Fuel & Trans & MPG & GHG & Oil Use \\
\hline$\overline{\mathrm{C} 4}$ & DCC - PLYMOUTH & BREEZE & $\mathrm{D}$ & $\mathrm{F}$ & $\mathrm{L} 4$ & 2.0 & $G$ & $\mathrm{M}$ & 35.4 & 5.4 & 10.1 \\
\hline C3 & DCC - PLYMOUTH & NEON & D & $\mathrm{F}$ & L4 & 2.0 & G & A & 31.9 & 6.0 & 11.2 \\
\hline $\mathrm{C} 7$ & FMC - FORD & FOCUS WAGON & $\mathrm{D}$ & $\mathrm{F}$ & L4 & 2.0 & G & M & 36.2 & 5.3 & 9.9 \\
\hline C3 & FMC - FORD & FOCUS & $\mathrm{D}$ & $\mathrm{F}$ & L4 & 2.0 & G & M & 35.7 & 5.4 & 10.0 \\
\hline C3 & FMC - FORD & ESCORT & $\mathrm{D}$ & $\mathrm{F}$ & L4 & 2.0 & G & M & 35.7 & 5.4 & 10.0 \\
\hline C3 & FMC - FORD & CONTOUR & $D$ & $\mathrm{~F}$ & L4 & 2.0 & G & M & 32.6 & 5.9 & 11.0 \\
\hline C5 & FMC - FORD & TAURUS & D & $\mathrm{F}$ & V6 & 3.0 & G & A & 26.3 & 7.2 & 13.6 \\
\hline C4 & FMC - JAGUAR & JAGUAR S-TYPE 6 & I & $\mathrm{R}$ & V6 & 3.0 & G & A & 24.1 & 7.9 & 14.8 \\
\hline C1 & FMC - JAGUAR & $\begin{array}{l}\text { JAGUAR XK8 } \\
\text { CONVERT. }\end{array}$ & I & $\mathrm{R}$ & V8 & 4.0 & G & A & 22.8 & 8.3 & 15.7 \\
\hline C2 & FMC - JAGUAR & $\begin{array}{l}\text { JAGUAR XK8 } \\
\text { COUPE }\end{array}$ & I & $\mathrm{R}$ & V8 & 4.0 & G & A & 23.1 & 8.2 & 15.4 \\
\hline C3 & FMC - JAGUAR & JAGUAR XJ8L & I & $\mathrm{R}$ & V8 & 4.0 & G & A & 22.8 & 8.3 & 15.7 \\
\hline C3 & FMC - JAGUAR & JAGUAR XJ8 & I & $\mathrm{R}$ & V8 & 4.0 & G & A & 22.8 & 8.3 & 15.7 \\
\hline C4 & FMC - JAGUAR & JAGUAR VDP & I & $\mathrm{R}$ & V8 & 4.0 & G & A & 22.8 & 8.3 & 15.7 \\
\hline C1 & FMC - JAGUAR & $\begin{array}{l}\text { JAGUAR XKR } \\
\text { CONVERT. }\end{array}$ & I & $\mathrm{R}$ & V8 & 4.0 & G & A & 21.4 & 8.8 & 16.7 \\
\hline C2 & FMC - JAGUAR & $\begin{array}{l}\text { JAGUAR XKR } \\
\text { COUPE }\end{array}$ & I & $\mathrm{R}$ & V8 & 4.0 & G & A & 21.9 & 8.7 & 16.3 \\
\hline C3 & FMC - JAGUAR & JAGUAR XJR & I & $\mathrm{R}$ & V8 & 4.0 & G & A & 21.1 & 9.0 & 17.0 \\
\hline C4 & FMC - JAGUAR & JAGUAR VDP S/C & I & $\mathrm{R}$ & V8 & 4.0 & G & A & 20.9 & 9.0 & 17.1 \\
\hline C5 & FMC - LINCOLN & CONTINENTAL & $\mathrm{D}$ & $\mathrm{F}$ & V8 & 4.6 & G & A & 23.4 & 8.1 & 15.3 \\
\hline C4 & FMC - MAZDA & 626 & I & $\mathrm{F}$ & L4 & 2.0 & G & M & 33.5 & 5.7 & 10.6 \\
\hline C3 & FMC - MERCURY & COUGAR & $\mathrm{D}$ & $\mathrm{F}$ & L4 & 2.0 & G & M & 32.4 & 5.9 & 11.0 \\
\hline C4 & FMC - MERCURY & SABLE & $\mathrm{D}$ & $\mathrm{F}$ & V6 & 3.0 & G & A & 26.4 & 7.2 & 13.5 \\
\hline C3 & FMC - VOLVO & C70 COUPE & 1 & $\mathrm{~F}$ & L5 & 2.3 & G & M & 26.9 & 7.1 & 13.3 \\
\hline C4 & FMC - VOLVO & $\mathrm{S} 70$ & I & $\mathrm{F}$ & L5 & 2.3 & G & M & 26.8 & 7.1 & 13.3 \\
\hline $\mathrm{C} 2$ & FMC - VOLVO & C70 CONVERTIBLE & I & $\mathrm{F}$ & L5 & 2.3 & G & M & 26.1 & 7.3 & 13.7 \\
\hline C3 & FMC - VOLVO & C70 COUPE & I & $\mathrm{F}$ & L5 & 2.3 & G & A & 25.7 & 7.4 & 13.9 \\
\hline C4 & FMC - VOLVO & $\mathrm{S} 70$ & I & $\mathrm{F}$ & L5 & 2.3 & G & A & 26.4 & 7.2 & 13.5 \\
\hline C8 & FMC - VOLVO & V70 & I & $\mathrm{F}$ & L5 & 2.3 & G & M & 26.8 & 7.1 & 13.3 \\
\hline C4 & FMC - VOLVO & S80 T6 & I & $\mathrm{F}$ & L6 & 2.8 & G & A & 23.3 & 8.1 & 15.3 \\
\hline C4 & GMC - BUICK & CENTURY & D & $\mathrm{F}$ & V6 & 3.1 & G & A & 27.5 & 6.9 & 13.0 \\
\hline C4 & GMC - BUICK & REGAL & $\mathrm{D}$ & $\mathrm{F}$ & V6 & 3.8 & G & A & 26.9 & 7.1 & 13.3 \\
\hline C5 & GMC - BUICK & LESABRE & $\mathrm{D}$ & $\mathrm{F}$ & V6 & 3.8 & G & A & 26.6 & 7.2 & 13.5 \\
\hline C5 & GMC - BUICK & PARK AVENUE & $\mathrm{D}$ & $\mathrm{F}$ & V6 & 3.8 & G & A & 26.6 & 7.2 & 13.5 \\
\hline C4 & GMC - CADILLAC & ELDORADO & $\mathrm{D}$ & $\mathrm{F}$ & V8 & 4.6 & G & A & 24.1 & 7.9 & 14.8 \\
\hline C4 & GMC - CADILLAC & SEVILLE & $\mathrm{D}$ & $\mathrm{F}$ & V8 & 4.6 & G & A & 24.1 & 7.9 & 14.8 \\
\hline C5 & GMC - CADILLAC & DEVILLE & D & $\mathrm{F}$ & V8 & 4.6 & G & A & 24.1 & 7.9 & 14.8 \\
\hline C2 & GMC - CHEVY & METRO & 1 & $\mathrm{~F}$ & L4 & 1.3 & G & M & 45.5 & 4.2 & 7.8 \\
\hline C3 & GMC - CHEVY & PRIZM & 1 & $\mathrm{~F}$ & L4 & 1.8 & G & M & 39.6 & 4.9 & 9.0 \\
\hline C3 & GMC - CHEVY & PRIZM & I & $\mathrm{F}$ & L4 & 1.8 & G & A & 37.5 & 5.1 & 9.5 \\
\hline C3 & GMC - CHEVY & PRIZM & 1 & $\mathrm{~F}$ & L4 & 1.8 & G & A & 34.9 & 5.5 & 10.2 \\
\hline C4 & GMC - CHEVY & MONTECARLO & D & $\mathrm{F}$ & V6 & 3.4 & G & A & 27.5 & 6.9 & 13.0 \\
\hline C5 & GMC - CHEVY & IMPALA & $\mathrm{D}$ & $\mathrm{F}$ & V6 & 3.4 & G & A & 27.5 & 6.9 & 13.0 \\
\hline $\mathrm{C} 2$ & GMC - CHEVY & CAMARO & $\mathrm{D}$ & $\mathrm{R}$ & V6 & 3.8 & G & M & 26.8 & 7.1 & 13.3 \\
\hline C4 & GMC - CHEVY & MONTECARLO & D & $\mathrm{F}$ & V6 & 3.8 & G & A & 26.9 & 7.1 & 13.3 \\
\hline C5 & GMC - CHEVY & IMPALA & $\mathrm{D}$ & $\mathrm{F}$ & V6 & 3.8 & G & A & 26.9 & 7.1 & 13.3 \\
\hline $\mathrm{C} 2$ & GMC - CHEVY & CAMARO & D & $\mathrm{R}$ & V8 & 5.7 & G & $M$ & 25.1 & 7.6 & 14.3 \\
\hline
\end{tabular}




\begin{tabular}{|c|c|c|c|c|c|c|c|c|c|c|c|}
\hline $\begin{array}{l}\text { Type-Size } \\
\text { Class }\end{array}$ & Manufacturer/Division & Name & $\begin{array}{l}\text { Dom I } \\
\text { Imp }\end{array}$ & DR & Cyl & Disp $F$ & Fuel & Trans & MPG & GHG & Oil Use \\
\hline $\mathrm{C} 6$ & GMC - CHEVY & CORVETTE & $\mathrm{D}$ & $\mathrm{R}$ & V8 & 5.7 & $\mathrm{G}$ & $\mathrm{M}$ & 25.1 & 7.6 & 14.2 \\
\hline $\mathrm{C} 2$ & GMC - CHEVY & CAMARO & $\mathrm{D}$ & $\mathrm{R}$ & V8 & 5.7 & G & $A$ & 22.8 & 8.3 & 15.7 \\
\hline $\mathrm{C} 6$ & GMC - CHEVY & CORVETTE & $\mathrm{D}$ & $\mathrm{R}$ & V8 & 5.7 & G & A & 22.8 & 8.3 & 15.7 \\
\hline $\mathrm{C} 4$ & GMC - OLDSMOBILE & INTRIGUE & $D$ & $\mathrm{~F}$ & V6 & 3.5 & G & A & 25.7 & 7.4 & 13.9 \\
\hline $\mathrm{C} 2$ & GMC - PONTIAC & SUNFIRE & $\mathrm{D}$ & $\mathrm{F}$ & L4 & 2.4 & G & M & 30.8 & 6.2 & 11.6 \\
\hline $\mathrm{C} 2$ & GMC - PONTIAC & FIREBIRD & D & $\mathrm{R}$ & V6 & 3.8 & G & M & 26.8 & 7.1 & 13.3 \\
\hline $\mathrm{C} 4$ & GMC - PONTIAC & GRAND PRIX & D & $\mathrm{F}$ & V6 & 3.8 & G & A & 26.9 & 7.1 & 13.3 \\
\hline $\mathrm{C} 2$ & GMC - PONTIAC & FIREBIRD & D & $\mathrm{R}$ & V6 & 3.8 & G & A & 26.1 & 7.3 & 13.7 \\
\hline C5 & GMC - PONTIAC & BONNEVILLE & $\mathrm{D}$ & $\mathrm{F}$ & V6 & 3.8 & G & A & 26.6 & 7.2 & 13.5 \\
\hline $\mathrm{C} 2$ & GMC - PONTIAC & FIREBIRD & D & $\mathrm{R}$ & V8 & 5.7 & G & $\mathrm{M}$ & 24.8 & 7.7 & 14.4 \\
\hline C5 & GMC - PONTIAC & BONNEVILLE & D & $\mathrm{F}$ & V6 & 3.8 & G & A & 24.8 & 7.7 & 14.4 \\
\hline $\mathrm{C} 2$ & GMC - SAAB & SAAB 9-3 CVT & I & $\mathrm{F}$ & L4 & 2.0 & G & M & 28.6 & 6.7 & 12.5 \\
\hline $\mathrm{C} 4$ & GMC - SAAB & SAAB 9-3 & I & $\mathrm{F}$ & L4 & 2.0 & G & M & 28.6 & 6.7 & 12.5 \\
\hline $\mathrm{C} 4$ & GMC - SAAB & SAAB 9-3 VIGGEN & I & $\mathrm{F}$ & L4 & 2.3 & G & $M$ & 27.5 & 6.9 & 13.0 \\
\hline $\mathrm{C} 4$ & GMC - SAAB & SAAB 9-3 VIGGEN & I & $\mathrm{F}$ & L4 & 2.3 & G & M & 27.5 & 6.9 & 13.0 \\
\hline C4 & GMC - SAAB & SAAB 9-5 & 1 & $\mathrm{~F}$ & L4 & 2.3 & G & M & 27.6 & 6.9 & 12.9 \\
\hline C3 & GMC - SATURN & SL & D & $\mathrm{F}$ & L4 & 1.9 & G & $M$ & 38.7 & 5.0 & 9.2 \\
\hline $\mathrm{C} 2$ & GMC - SATURN & SC & D & $\mathrm{F}$ & L4 & 1.9 & G & $M$ & 35.4 & 5.4 & 10.1 \\
\hline $\mathrm{C} 2$ & GMC - SATURN & SC & $\mathrm{D}$ & $\mathrm{F}$ & L4 & 1.9 & G & $M$ & 38.1 & 5.0 & 9.4 \\
\hline C3 & GMC - SATURN & SL & $D$ & $\mathrm{~F}$ & L4 & 1.9 & G & $\mathrm{M}$ & 36.1 & 5.3 & 9.9 \\
\hline $\mathrm{C} 7$ & GMC - SATURN & SW & $\mathrm{D}$ & $\mathrm{F}$ & L4 & 1.9 & G & M & 36.1 & 5.3 & 9.9 \\
\hline $\mathrm{C} 3$ & GMC - SATURN & SL & $\mathrm{D}$ & $\mathrm{F}$ & L4 & 1.9 & G & A & 34.4 & 5.6 & 10.4 \\
\hline $\mathrm{C} 7$ & GMC - SATURN & SW & D & $\mathrm{F}$ & L4 & 1.9 & G & A & 35.4 & 5.4 & 10.1 \\
\hline $\mathrm{C} 4$ & GMC - SATURN & LS & D & $\mathrm{F}$ & L4 & 2.2 & G & M & 31.9 & 6.0 & 11.2 \\
\hline $\mathrm{C} 4$ & GMC - SATURN & LS & $\mathrm{D}$ & $\mathrm{F}$ & L4 & 2.2 & G & A & 30.5 & 6.3 & 11.7 \\
\hline $\mathrm{C} 2$ & HONDA - ACURA & ACURA INTEGRA -R & 1 & $\mathrm{~F}$ & L4 & 1.8 & G & M & 31.9 & 6.0 & 11.2 \\
\hline $\mathrm{C} 4$ & HONDA - ACURA & ACURA 3.2TL & I & $\mathrm{F}$ & V6 & 3.2 & G & $A$ & 26.4 & 7.2 & 13.5 \\
\hline $\mathrm{C} 6$ & HONDA & INSIGHT & I & $\mathrm{F}$ & L3 & 1.0 & G/EL & M & 76.3 & 2.6 & 4.7 \\
\hline $\mathrm{C} 2$ & HONDA & CIVIC HX & I & $\mathrm{F}$ & L4 & 1.6 & G & M & 44.7 & 4.3 & 8.0 \\
\hline $\mathrm{C} 2$ & HONDA & CIVIC HX & I & $F$ & L4 & 1.6 & G & $A$ & 41.9 & 4.6 & 8.5 \\
\hline $\mathrm{C} 2$ & HONDA & CIVIC dx 4Dr & I & $\mathrm{F}$ & L4 & 1.6 & G & M & 37.2 & 5.2 & 9.6 \\
\hline $\mathrm{C} 2$ & HONDA & CIVIC ex & I & $\mathrm{F}$ & L4 & 1.6 & G & M & 37.2 & 5.2 & 9.6 \\
\hline $\mathrm{C} 2$ & HONDA & CIVIC ex & I & $\mathrm{F}$ & L4 & 1.6 & G & A & 35.5 & 5.4 & 10.1 \\
\hline $\mathrm{C} 2$ & HONDA & CIVIC si & I & $\mathrm{F}$ & L4 & 1.6 & G & $M$ & 32.8 & 5.8 & 10.9 \\
\hline $\mathrm{C} 4$ & HONDA & ACCORD Ix & I & $\mathrm{F}$ & L4 & 2.3 & G & M & 32.1 & 6.0 & 11.1 \\
\hline C4 & HONDA & ACCORD Ix & I & $\mathrm{F}$ & L4 & 2.3 & G & A & 29.9 & 6.4 & 11.9 \\
\hline $\mathrm{C} 2$ & HONDA & PRELUDE & I & $F$ & L4 & 2.2 & G & $M$ & 28.2 & 6.8 & 12.7 \\
\hline $\mathrm{C} 2$ & HONDA & PRELUDE & I & $\mathrm{F}$ & L4 & 2.2 & G & A & 28.3 & 6.7 & 12.6 \\
\hline $\mathrm{C} 4$ & HONDA & ACCORD coupe & I & $\mathrm{F}$ & V6 & 3.0 & G & A & 27.0 & 7.0 & 13.2 \\
\hline $\mathrm{C} 2$ & MITSUBISHI & MIRAGE 2D & I & $\mathrm{F}$ & L4 & 1.5 & G & M & 41.7 & 4.6 & 8.6 \\
\hline $\mathrm{C} 2$ & MITSUBISHI & ECLIPSE & I & $\mathrm{F}$ & V6 & 3.0 & G & M & 27.2 & 7.0 & 13.1 \\
\hline $\mathrm{C} 2$ & MITSUBISHI & ECLIPSE & I & $\mathrm{F}$ & V6 & 3.0 & G & A & 27.8 & 6.8 & 12.8 \\
\hline C3 & NISSAN - INFINITY & INFINITI G20 & 1 & $\mathrm{~F}$ & L4 & 2.0 & G & M & 31.0 & 6.2 & 11.5 \\
\hline $\mathrm{C} 4$ & NISSAN - INFINITY & INFINITI I30 & I & $\mathrm{F}$ & V6 & 3.0 & G & A & 26.4 & 7.2 & 13.5 \\
\hline $\mathrm{C} 4$ & NISSAN - INFINITY & INFINITI Q45 & I & $\mathrm{R}$ & V8 & 4.1 & G & A & 23.1 & 8.2 & 15.5 \\
\hline C3 & NISSAN & SENTRA & I & $\mathrm{F}$ & L4 & 1.8 & G & M & 34.9 & 5.5 & 10.2 \\
\hline C3 & NISSAN & ALTIMA & 1 & $\mathrm{~F}$ & L4 & 2.4 & $G$ & $\mathrm{M}$ & 30.9 & 6.2 & 11.6 \\
\hline
\end{tabular}




\begin{tabular}{|c|c|c|c|c|c|c|c|c|c|c|c|}
\hline $\begin{array}{l}\text { Type-Size } \\
\text { Class }\end{array}$ & Manufacturer/Division & Name & $\begin{array}{l}\text { Dom I } \\
\text { Imp }\end{array}$ & DR & Cyl & Disp & Fuel & Trans & MPG & GHG & Oil Use \\
\hline$\overline{\mathrm{C} 4}$ & NISSAN & MAXIMA & $\mathrm{I}$ & $F$ & L6 & 3.0 & $\mathrm{G}$ & $\mathrm{M}$ & 27.8 & 6.9 & 12.8 \\
\hline $\mathrm{C} 2$ & SUZUKI & SWIFT & I & $\mathrm{F}$ & L4 & 1.3 & $\mathrm{G}$ & $M$ & 45.5 & 4.2 & 7.8 \\
\hline $\mathrm{C} 2$ & TOYOTA - LEXUS & SC 400 & I & $\mathrm{R}$ & V8 & 4.0 & G & $A$ & 24.3 & 7.8 & 14.7 \\
\hline $\mathrm{C} 4$ & TOYOTA - LEXUS & LS 400 & I & $\mathrm{R}$ & V8 & 4.0 & G & $A$ & 24.3 & 7.8 & 14.7 \\
\hline $\mathrm{C} 4$ & TOYOTA - LEXUS & GS 400 & 1 & $\mathrm{R}$ & V8 & 4.0 & G & A & 23.3 & 8.2 & 15.4 \\
\hline $\mathrm{C} 3$ & TOYOTA & $\mathrm{ECHO}$ & I & $\mathrm{F}$ & L4 & 1.5 & G & M & 43.1 & 4.5 & 8.3 \\
\hline C3 & TOYOTA & COROLLA & I & $\mathrm{F}$ & L4 & 1.8 & $\mathrm{G}$ & $M$ & 39.6 & 4.9 & 9.0 \\
\hline $\mathrm{C} 3$ & TOYOTA & $\mathrm{ECHO}$ & I & $\mathrm{F}$ & L4 & 1.5 & $\mathrm{G}$ & A & 39.4 & 4.9 & 9.1 \\
\hline $\mathrm{C} 3$ & TOYOTA & COROLLA & I & $F$ & L4 & 1.8 & G & A & 37.4 & 5.1 & 9.6 \\
\hline $\mathrm{C} 2$ & TOYOTA & CELICA & I & $\mathrm{F}$ & L4 & 1.8 & $\mathrm{G}$ & M & 35.2 & 5.4 & 10.1 \\
\hline $\mathrm{C} 2$ & TOYOTA & CELICA & I & $F$ & L4 & 1.8 & G & $A$ & 34.9 & 5.5 & 10.2 \\
\hline $\mathrm{C} 3$ & TOYOTA & COROLLA & I & $\mathrm{F}$ & L4 & 1.8 & $\mathrm{G}$ & $A$ & 34.9 & 5.5 & 10.2 \\
\hline $\mathrm{C} 3$ & TOYOTA & CAMRY SOLARA & I & $\mathrm{F}$ & L4 & 2.2 & G & M & 31.0 & 6.2 & 11.5 \\
\hline $\mathrm{C} 2$ & TOYOTA & CELICA & I & $F$ & L4 & 1.8 & G & M & 30.8 & 6.2 & 11.6 \\
\hline $\mathrm{C} 2$ & TOYOTA & $\begin{array}{l}\text { CAMRY SOLARA } \\
\text { CONVERT }\end{array}$ & I & $F$ & L4 & 2.2 & G & $A$ & 30.1 & 6.4 & 11.9 \\
\hline C5 & TOYOTA & AVALON & I & $\mathrm{F}$ & V6 & 3.0 & $\mathrm{G}$ & $A$ & 28.1 & 6.8 & 12.7 \\
\hline C3 & VWA - AUDI & AUDI A4 & 1 & $F$ & L4 & 1.8 & G & M & 31.9 & 6.0 & 11.2 \\
\hline $\mathrm{C} 1$ & VWA - AUDI & AUDI TT COUPE & I & 4 & L4 & 1.8 & G & $M$ & 29.4 & 6.5 & 12.1 \\
\hline C5 & VWA - AUDI & AUDI A8 & I & 4 & V8 & 4.2 & $\mathrm{G}$ & A & 22.7 & 8.3 & 15.7 \\
\hline $\mathrm{C} 4$ & VWA - AUDI & AUDI A6 & I & 4 & V8 & 4.2 & G & A & 22.7 & 8.3 & 15.7 \\
\hline C5 & VWA - AUDI & AUDI A8 (LWB) & I & 4 & V8 & 4.2 & $\mathrm{G}$ & $A$ & 22.8 & 8.3 & 15.7 \\
\hline $\mathrm{C} 2$ & VWA & NEW BEETLE & 1 & $F$ & L4 & 1.9 & $\mathrm{D}$ & M & 52.7 & 4.0 & 6.8 \\
\hline $\mathrm{C} 3$ & VWA & GOLF TDI & I & $F$ & L4 & 1.9 & $\mathrm{D}$ & M & 52.7 & 4.0 & 6.8 \\
\hline $\mathrm{C} 3$ & VWA & JETTA & I & $F$ & L4 & 1.9 & $\mathrm{D}$ & M & 52.7 & 4.0 & 6.8 \\
\hline $\mathrm{C} 2$ & VWA & NEW BEETLE & I & $F$ & L4 & 1.9 & $\mathrm{D}$ & A & 44.7 & 4.7 & 8.0 \\
\hline C3 & VWA & GOLF TDI & I & $\mathrm{F}$ & L4 & 1.9 & $\mathrm{D}$ & $A$ & 44.7 & 4.7 & 8.0 \\
\hline C3 & VWA & JETTA & 1 & $F$ & L4 & 1.9 & $\mathrm{D}$ & A & 44.7 & 4.7 & 8.0 \\
\hline $\mathrm{C} 2$ & VWA & NEW BEETLE & 1 & $F$ & L4 & 1.8 & $\mathrm{G}$ & $M$ & 32.3 & 5.9 & 11.1 \\
\hline C3 & VWA & GTI & I & $F$ & L4 & 1.8 & G & $\mathrm{M}$ & 31.3 & 6.1 & 11.4 \\
\hline $\mathrm{C} 3$ & VWA & GOLF & I & $F$ & L4 & 1.8 & G & M & 31.3 & 6.1 & 11.4 \\
\hline C3 & VWA & JETTA & I & $\mathrm{F}$ & L4 & 1.8 & $\mathrm{G}$ & $M$ & 31.3 & 6.1 & 11.4 \\
\hline $\mathrm{C} 4$ & VWA & PASSAT & 1 & $F$ & L4 & 1.8 & G & M & 31.3 & 6.1 & 11.4 \\
\hline $\mathrm{C} 8$ & VWA & PASSAT WAGON & I & $\mathrm{F}$ & L4 & 1.8 & G & $M$ & 31.3 & 6.1 & 11.4 \\
\hline \multicolumn{12}{|l|}{ Trucks } \\
\hline T3 & HONDA & ODYSSEY & I & $\mathrm{F}$ & V6 & 3.5 & $\mathrm{G}$ & $A$ & 24.0 & 7.9 & 14.9 \\
\hline
\end{tabular}


Energy Star Method 7 Vehicle List

Top 20\% of vehicles by Ratio of Actual to Predicted MPG (equation 2)

(MPG Predicted by Regression Model Using Curb Weight, Horsepower, and Car/Truck)

\begin{tabular}{|c|c|c|c|c|c|c|c|c|c|c|c|}
\hline $\begin{array}{l}\text { Type- } \\
\text { Size } \\
\text { Class } \\
\end{array}$ & Manufacturer/Division & Name & $\begin{array}{c}\text { Dom I } \\
\text { Imp }\end{array}$ & DR & Cyl & Disp & Fuel & Trans & MPG & GHG & Oil Use \\
\hline \multicolumn{12}{|l|}{ Cars } \\
\hline $\mathrm{C} 3$ & BMW & $528 \mathrm{i}$ & 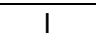 & $\mathrm{R}$ & L6 & 2.8 & $\mathrm{G}$ & $M$ & 27.6 & 6.9 & $\overline{12.9}$ \\
\hline C3 & BMW & $540 \mathrm{iA}$ & I & $\mathrm{R}$ & V8 & 4.4 & G & $A$ & 23.7 & 8.0 & 15.1 \\
\hline C4 & BMW & $740 \mathrm{iA}$ & 1 & $\mathrm{R}$ & V8 & 4.4 & G & $A$ & 22.7 & 8.4 & 15.8 \\
\hline C5 & BMW & 740iLA & 1 & $\mathrm{R}$ & V8 & 4.4 & G & $A$ & 22.7 & 8.4 & 15.8 \\
\hline C3 & DCC - CHRYSLER & NEON & $\mathrm{D}$ & $\mathrm{F}$ & L4 & 2.0 & $\mathrm{G}$ & M & 36.1 & 5.3 & 9.9 \\
\hline C3 & DCC - CHRYSLER & NEON & $\mathrm{D}$ & $\mathrm{F}$ & L4 & 2.0 & G & $A$ & 31.9 & 6.0 & 11.2 \\
\hline C5 & DCC - CHRYSLER & INTREPID & $\mathrm{D}$ & $\mathrm{F}$ & V6 & 2.7 & G & $A$ & 27.3 & 7.0 & 13.1 \\
\hline C5 & DCC - CHRYSLER & CONCORDE & $\mathrm{D}$ & $\mathrm{F}$ & V6 & 2.7 & $\mathrm{G}$ & $A$ & 27.3 & 7.0 & 13.1 \\
\hline C5 & DCC - CHRYSLER & CONCORDE & $\mathrm{D}$ & $\mathrm{F}$ & V6 & 3.2 & $\mathrm{G}$ & $A$ & 25.4 & 7.5 & 14.1 \\
\hline C5 & DCC - CHRYSLER & $300 \mathrm{M}$ & $\mathrm{D}$ & $\mathrm{F}$ & V6 & 3.5 & G & $A$ & 24.8 & 7.7 & 14.4 \\
\hline C3 & DCC - DODGE & NEON & $\mathrm{D}$ & $\mathrm{F}$ & L4 & 2.0 & G & $M$ & 36.1 & 5.3 & 9.9 \\
\hline C3 & DCC - DODGE & NEON & $\mathrm{D}$ & $\mathrm{F}$ & L4 & 2.0 & G & A & 31.9 & 6.0 & 11.2 \\
\hline C5 & DCC - DODGE & INTREPID & D & $\mathrm{F}$ & V6 & 2.7 & G & $A$ & 27.3 & 7.0 & 13.1 \\
\hline C5 & DCC - DODGE & INTREPID & $\mathrm{D}$ & $\mathrm{F}$ & V6 & 2.7 & $\mathrm{G}$ & $A$ & 27.3 & 7.0 & 13.1 \\
\hline C5 & DCC - DODGE & INTREPID & $\mathrm{D}$ & $\mathrm{F}$ & V6 & 3.5 & $\mathrm{G}$ & $A$ & 24.8 & 7.7 & 14.4 \\
\hline C3 & DCC - MER-BENZ & C 230 KOMPRESSOR & 1 & $\mathrm{R}$ & L4 & 2.3 & G & $A$ & 28.3 & 6.7 & 12.6 \\
\hline C6 & DCC - MER-BENZ & $\begin{array}{l}\text { SLK } 230 \\
\text { KOMPRESSOR }\end{array}$ & I & $\mathrm{R}$ & L4 & 2.3 & G & $A$ & 29.2 & 6.5 & 12.2 \\
\hline $\mathrm{C} 1$ & DCC - MER-BENZ & CLK 320 (CABRIOLET) & I & $\mathrm{R}$ & V6 & 3.2 & $\mathrm{G}$ & $A$ & 26.5 & 7.2 & 13.5 \\
\hline C4 & DCC - MER-BENZ & E 320 & I & $\mathrm{R}$ & V6 & 3.2 & G & $A$ & 27.9 & 6.8 & 12.8 \\
\hline C8 & DCC - MER-BENZ & E 320 (WAGON) & I & $\mathrm{R}$ & V6 & 3.2 & $\mathrm{G}$ & $A$ & 26.8 & 7.1 & 13.3 \\
\hline $\mathrm{C} 2$ & DCC - MER-BENZ & CLK 320 & I & $\mathrm{R}$ & V6 & 3.2 & G & $A$ & 27.5 & 6.9 & 13.0 \\
\hline C4 & DCC - MER-BENZ & E 320 4MATIC & I & 4 & V6 & 3.2 & G & $A$ & 26.9 & 7.1 & 13.3 \\
\hline C8 & DCC - MER-BENZ & $\begin{array}{l}\text { E } 320 \\
\text { 4MATIC(WAGON) }\end{array}$ & I & 4 & V6 & 3.2 & $\mathrm{G}$ & $A$ & 25.9 & 7.3 & 13.8 \\
\hline C4 & DCC - MER-BENZ & E 430 & I & $\mathrm{R}$ & V8 & 4.3 & G & $A$ & 23.9 & 7.9 & 14.9 \\
\hline C5 & DCC - MER-BENZ & S 430 & I & $\mathrm{R}$ & V8 & 4.3 & G & $A$ & 23.0 & 8.2 & 15.5 \\
\hline C4 & DCC - MER-BENZ & E 55 AMG & I & $\mathrm{R}$ & V8 & 5.5 & $\mathrm{G}$ & $A$ & 22.0 & 8.6 & 16.2 \\
\hline C3 & DCC - PLYMOUTH & NEON & D & $\mathrm{F}$ & L4 & 2.0 & $\mathrm{G}$ & $M$ & 36.1 & 5.3 & 9.9 \\
\hline C4 & DCC - PLYMOUTH & BREEZE & $\mathrm{D}$ & $\mathrm{F}$ & L4 & 2.0 & $\mathrm{G}$ & $M$ & 35.4 & 5.4 & 10.1 \\
\hline C3 & DCC - PLYMOUTH & NEON & $\mathrm{D}$ & $\mathrm{F}$ & $\mathrm{L} 4$ & 2.0 & $\mathrm{G}$ & $A$ & 31.9 & 6.0 & 11.2 \\
\hline $\mathrm{C} 7$ & FMC - FORD & FOCUS WAGON & $\mathrm{D}$ & $\mathrm{F}$ & $\mathrm{L} 4$ & 2.0 & G & M & 36.2 & 5.3 & 9.9 \\
\hline C3 & FMC - FORD & CONTOUR & $\mathrm{D}$ & $\mathrm{F}$ & L4 & 2.0 & G & M & 32.6 & 5.9 & 11.0 \\
\hline $\mathrm{C} 1$ & FMC - JAGUAR & $\begin{array}{l}\text { JAGUAR XK8 } \\
\text { CONVERT. }\end{array}$ & I & $\mathrm{R}$ & V8 & 4.0 & $\mathrm{G}$ & $A$ & 22.8 & 8.3 & 15.7 \\
\hline $\mathrm{C} 2$ & FMC - JAGUAR & JAGUAR XK8 COUPE & 1 & $\mathrm{R}$ & V8 & 4.0 & G & $A$ & 23.1 & 8.2 & 15.4 \\
\hline C3 & FMC - JAGUAR & JAGUAR XJ8L & 1 & $\mathrm{R}$ & V8 & 4.0 & G & $A$ & 22.8 & 8.3 & 15.7 \\
\hline C3 & FMC - JAGUAR & JAGUAR XJ8 & 1 & $\mathrm{R}$ & V8 & 4.0 & G & $A$ & 22.8 & 8.3 & 15.7 \\
\hline C4 & FMC - JAGUAR & JAGUAR VDP & 1 & $\mathrm{R}$ & V8 & 4.0 & G & $A$ & 22.8 & 8.3 & 15.7 \\
\hline $\mathrm{C} 1$ & FMC - JAGUAR & $\begin{array}{l}\text { JAGUAR XKR } \\
\text { CONVERT. }\end{array}$ & I & $\mathrm{R}$ & V8 & 4.0 & $\mathrm{G}$ & $A$ & 21.4 & 8.8 & 16.7 \\
\hline $\mathrm{C} 2$ & FMC - JAGUAR & JAGUAR XKR COUPE & 1 & $\mathrm{R}$ & V8 & 4.0 & G & A & 21.9 & 8.7 & 16.3 \\
\hline C3 & FMC - JAGUAR & JAGUAR XJR & I & $\mathrm{R}$ & V8 & 4.0 & G & $A$ & 21.1 & 9.0 & 17.0 \\
\hline C4 & FMC - JAGUAR & JAGUAR VDP S/C & 1 & $\mathrm{R}$ & V8 & 4.0 & G & $A$ & 20.9 & 9.0 & 17.1 \\
\hline C5 & FMC - LINCOLN & CONTINENTAL & D & $\mathrm{F}$ & V8 & 4.6 & $\mathrm{G}$ & $A$ & 23.4 & 8.1 & 15.3 \\
\hline
\end{tabular}




\begin{tabular}{|c|c|c|c|c|c|c|c|c|c|c|c|}
\hline $\begin{array}{l}\text { Type- } \\
\text { Size } \\
\text { Class }\end{array}$ & Manufacturer/Division & Name & $\begin{array}{c}\text { Dom I } \\
\text { Imp }\end{array}$ & DR & Cyl & Disp & Fuel & Trans & MPG & GHG & Oil Use \\
\hline$\overline{\mathrm{C} 4}$ & FMC - MAZDA & 626 & $\mathrm{I}$ & $\bar{F}$ & $\mathrm{~L} 4$ & 2.0 & $\bar{G}$ & $\mathrm{M}$ & 33.5 & 5.7 & 10.6 \\
\hline $\mathrm{C} 3$ & FMC - VOLVO & C70 COUPE & 1 & $F$ & L5 & 2.3 & G & $M$ & 26.9 & 7.1 & 13.3 \\
\hline $\mathrm{C} 4$ & FMC - VOLVO & S70 & I & $F$ & L5 & 2.3 & G & M & 26.8 & 7.1 & 13.3 \\
\hline $\mathrm{C} 2$ & FMC - VOLVO & C70 CONVERTIBLE & I & $F$ & L5 & 2.3 & G & M & 26.1 & 7.3 & 13.7 \\
\hline C3 & FMC - VOLVO & C70 COUPE & I & $\mathrm{F}$ & L5 & 2.3 & G & A & 25.7 & 7.4 & 13.9 \\
\hline $\mathrm{C} 4$ & FMC - VOLVO & S70 & I & $F$ & L5 & 2.3 & G & $A$ & 26.4 & 7.2 & 13.5 \\
\hline C8 & FMC - VOLVO & V70 & 1 & $F$ & L5 & 2.3 & G & M & 26.8 & 7.1 & 13.3 \\
\hline $\mathrm{C} 4$ & GMC - BUICK & REGAL & $\mathrm{D}$ & $F$ & V6 & 3.8 & G & $A$ & 26.9 & 7.1 & 13.3 \\
\hline C5 & GMC - BUICK & LESABRE & $D$ & $F$ & V6 & 3.8 & G & A & 26.6 & 7.2 & 13.5 \\
\hline C5 & GMC - BUICK & PARK AVENUE & $\mathrm{D}$ & $F$ & V6 & 3.8 & G & A & 26.6 & 7.2 & 13.5 \\
\hline $\mathrm{C} 4$ & GMC - CADILLAC & ELDORADO & $D$ & $F$ & V8 & 4.6 & G & $A$ & 24.1 & 7.9 & 14.8 \\
\hline C4 & GMC - CADILLAC & SEVILLE & $\mathrm{D}$ & $F$ & V8 & 4.6 & G & A & 24.1 & 7.9 & 14.8 \\
\hline C5 & GMC - CADILLAC & DEVILLE & $D$ & $F$ & V8 & 4.6 & G & A & 24.1 & 7.9 & 14.8 \\
\hline $\mathrm{C} 2$ & GMC - CHEVY & METRO & 1 & $F$ & L4 & 1.3 & G & M & 45.5 & 4.2 & 7.8 \\
\hline C3 & GMC - CHEVY & PRIZM & 1 & $F$ & L4 & 1.8 & G & M & 39.6 & 4.9 & 9.0 \\
\hline $\mathrm{C} 3$ & GMC - CHEVY & PRIZM & I & $F$ & $\mathrm{~L} 4$ & 1.8 & G & $A$ & 37.5 & 5.1 & 9.5 \\
\hline $\mathrm{C} 4$ & GMC - CHEVY & MONTECARLO & $D$ & $F$ & V6 & 3.8 & G & A & 26.9 & 7.1 & 13.3 \\
\hline C5 & GMC - CHEVY & IMPALA & $\mathrm{D}$ & $F$ & V6 & 3.8 & G & A & 26.9 & 7.1 & 13.3 \\
\hline $\mathrm{C} 2$ & GMC - CHEVY & CAMARO & $D$ & $\mathrm{R}$ & V8 & 5.7 & G & M & 25.1 & 7.6 & 14.3 \\
\hline $\mathrm{C} 6$ & GMC - CHEVY & CORVETTE & $\mathrm{D}$ & $\mathrm{R}$ & V8 & 5.7 & G & M & 25.1 & 7.6 & 14.2 \\
\hline $\mathrm{C} 4$ & GMC - PONTIAC & GRAND PRIX & $D$ & $F$ & V6 & 3.8 & G & $A$ & 26.9 & 7.1 & 13.3 \\
\hline $\mathrm{C} 2$ & GMC - PONTIAC & FIREBIRD & $\mathrm{D}$ & $\mathrm{R}$ & V6 & 3.8 & G & A & 26.1 & 7.3 & 13.7 \\
\hline C5 & GMC - PONTIAC & BONNEVILLE & $D$ & $F$ & V6 & 3.8 & G & A & 26.6 & 7.2 & 13.5 \\
\hline $\mathrm{C} 2$ & GMC - PONTIAC & FIREBIRD & $\mathrm{D}$ & $\mathrm{R}$ & V8 & 5.7 & G & M & 24.8 & 7.7 & 14.4 \\
\hline C5 & GMC - PONTIAC & BONNEVILLE & $\mathrm{D}$ & $F$ & V6 & 3.8 & G & A & 24.8 & 7.7 & 14.4 \\
\hline $\mathrm{C} 2$ & GMC - SAAB & SAAB 9-3 CVT & I & $F$ & $\mathrm{~L} 4$ & 2.0 & G & M & 28.6 & 6.7 & 12.5 \\
\hline $\mathrm{C} 4$ & GMC - SAAB & SAAB 9-3 VIGGEN & 1 & $F$ & L4 & 2.3 & G & M & 27.5 & 6.9 & 13.0 \\
\hline $\mathrm{C} 4$ & GMC - SAAB & SAAB 9-3 VIGGEN & 1 & $F$ & L4 & 2.3 & G & M & 27.5 & 6.9 & 13.0 \\
\hline C3 & GMC - SATURN & SL & $\mathrm{D}$ & $F$ & L4 & 1.9 & G & M & 38.7 & 5.0 & 9.2 \\
\hline $\mathrm{C} 7$ & GMC - SATURN & SW & $D$ & $F$ & $\mathrm{~L} 4$ & 1.9 & G & M & 36.1 & 5.3 & 9.9 \\
\hline $\mathrm{C} 7$ & GMC - SATURN & SW & $\mathrm{D}$ & $F$ & L4 & 1.9 & G & A & 35.4 & 5.4 & 10.1 \\
\hline C4 & GMC - SATURN & LS & $\mathrm{D}$ & $F$ & L4 & 2.2 & G & M & 31.9 & 6.0 & 11.2 \\
\hline $\mathrm{C} 2$ & HONDA - ACURA & ACURA INTEGRA -R & I & $F$ & L4 & 1.8 & G & M & 31.9 & 6.0 & 11.2 \\
\hline $\mathrm{C} 4$ & HONDA - ACURA & ACURA 3.2TL & 1 & $F$ & V6 & 3.2 & G & A & 26.4 & 7.2 & 13.5 \\
\hline C6 & HONDA & INSIGHT & I & $\mathrm{F}$ & L3 & 1.0 & $\mathrm{G} / \mathrm{EL}$ & M & 76.3 & 2.6 & 4.7 \\
\hline $\mathrm{C} 2$ & HONDA & CIVIC HX & I & $F$ & $\mathrm{~L} 4$ & 1.6 & G & M & 44.7 & 4.3 & 8.0 \\
\hline $\mathrm{C} 2$ & HONDA & CIVIC HX & 1 & $F$ & L4 & 1.6 & G & A & 41.9 & 4.6 & 8.5 \\
\hline $\mathrm{C} 2$ & HONDA & CIVIC ex & 1 & $\mathrm{~F}$ & L4 & 1.6 & G & M & 37.2 & 5.2 & 9.6 \\
\hline $\mathrm{C} 2$ & HONDA & CIVIC ex & 1 & $F$ & L4 & 1.6 & G & A & 35.5 & 5.4 & 10.1 \\
\hline $\mathrm{C} 2$ & HONDA & CIVIC si & 1 & $\mathrm{~F}$ & L4 & 1.6 & G & M & 32.8 & 5.8 & 10.9 \\
\hline $\mathrm{C} 4$ & HONDA & ACCORD Ix & I & $F$ & L4 & 2.3 & G & M & 32.1 & 6.0 & 11.1 \\
\hline $\mathrm{C} 2$ & HONDA & PRELUDE & I & $F$ & L4 & 2.2 & G & A & 28.3 & 6.7 & 12.6 \\
\hline $\mathrm{C} 2$ & MITSUBISHI & MIRAGE 2D & 1 & $F$ & L4 & 1.5 & G & M & 41.7 & 4.6 & 8.6 \\
\hline $\mathrm{C} 2$ & MITSUBISHI & ECLIPSE & 1 & $\mathrm{~F}$ & V6 & 3.0 & G & A & 27.8 & 6.8 & 12.8 \\
\hline C4 & NISSAN - INFINITY & INFINITI I30 & 1 & $F$ & V6 & 3.0 & G & A & 26.4 & 7.2 & 13.5 \\
\hline $\mathrm{C} 4$ & NISSAN - INFINITY & INFINITI Q45 & I & $\mathrm{R}$ & V8 & 4.1 & G & $A$ & 23.1 & 8.2 & 15.5 \\
\hline $\mathrm{C} 4$ & NISSAN & MAXIMA & I & $F$ & L6 & 3.0 & G & M & 27.8 & 6.9 & 12.8 \\
\hline
\end{tabular}




\begin{tabular}{|c|c|c|c|c|c|c|c|c|c|c|c|}
\hline $\begin{array}{l}\text { Type- } \\
\text { Size } \\
\text { Class }\end{array}$ & Manufacturer/Division & Name & $\begin{array}{l}\text { Dom I } \\
\text { Imp }\end{array}$ & DR & Cyl & Disp & Fuel & Trans & MPG & GHG & Oil Use \\
\hline$\overline{\mathrm{C} 2}$ & SUZUKI & SWIFT & $\mathrm{T}$ & $\bar{F}$ & $\mathrm{~L} 4$ & 1.3 & $\bar{G}$ & $\mathrm{M}$ & 45.5 & 4.2 & $\overline{7.8}$ \\
\hline $\mathrm{C} 2$ & TOYOTA - LEXUS & SC 400 & 1 & $\mathrm{R}$ & V8 & 4.0 & G & $A$ & 24.3 & 7.8 & 14.7 \\
\hline $\mathrm{C} 4$ & TOYOTA - LEXUS & LS 400 & I & $\mathrm{R}$ & V8 & 4.0 & G & $A$ & 24.3 & 7.8 & 14.7 \\
\hline $\mathrm{C} 4$ & TOYOTA - LEXUS & GS 400 & I & $\mathrm{R}$ & V8 & 4.0 & G & A & 23.3 & 8.2 & 15.4 \\
\hline C3 & TOYOTA & $\mathrm{ECHO}$ & I & $\mathrm{F}$ & L4 & 1.5 & G & M & 43.1 & 4.5 & 8.3 \\
\hline $\mathrm{C} 3$ & TOYOTA & COROLLA & I & $\mathrm{F}$ & L4 & 1.8 & G & M & 39.6 & 4.9 & 9.0 \\
\hline C3 & TOYOTA & COROLLA & I & $\mathrm{F}$ & L4 & 1.8 & G & A & 37.4 & 5.1 & 9.6 \\
\hline $\mathrm{C} 2$ & TOYOTA & CELICA & I & $\mathrm{F}$ & L4 & 1.8 & G & M & 35.2 & 5.4 & 10.1 \\
\hline $\mathrm{C} 2$ & TOYOTA & CELICA & I & $\mathrm{F}$ & L4 & 1.8 & G & A & 34.9 & 5.5 & 10.2 \\
\hline C5 & TOYOTA & AVALON & I & $\mathrm{F}$ & V6 & 3.0 & G & A & 28.1 & 6.8 & 12.7 \\
\hline $\mathrm{C} 3$ & VWA - AUDI & AUDI A4 & I & $\mathrm{F}$ & L4 & 1.8 & G & M & 31.9 & 6.0 & 11.2 \\
\hline C5 & VWA - AUDI & AUDI A8 & I & 4 & V8 & 4.2 & G & A & 22.7 & 8.3 & 15.7 \\
\hline $\mathrm{C} 4$ & VWA - AUDI & AUDI A6 & I & 4 & V8 & 4.2 & G & A & 22.7 & 8.3 & 15.7 \\
\hline C5 & VWA - AUDI & AUDI A8 (LWB) & I & 4 & V8 & 4.2 & G & A & 22.8 & 8.3 & 15.7 \\
\hline $\mathrm{C} 2$ & VWA & NEW BEETLE & I & $\mathrm{F}$ & L4 & 1.9 & $\mathrm{D}$ & M & 52.7 & 4.0 & 6.8 \\
\hline $\mathrm{C} 3$ & VWA & GOLF TDI & I & $\mathrm{F}$ & L4 & 1.9 & $D$ & M & 52.7 & 4.0 & 6.8 \\
\hline C3 & VWA & JETTA & I & $\mathrm{F}$ & L4 & 1.9 & D & M & 52.7 & 4.0 & 6.8 \\
\hline $\mathrm{C} 2$ & VWA & NEW BEETLE & I & $\mathrm{F}$ & L4 & 1.9 & $D$ & A & 44.7 & 4.7 & 8.0 \\
\hline $\mathrm{C} 3$ & VWA & GOLF TDI & I & $\mathrm{F}$ & L4 & 1.9 & $D$ & A & 44.7 & 4.7 & 8.0 \\
\hline C3 & VWA & JETTA & I & $\mathrm{F}$ & L4 & 1.9 & $D$ & A & 44.7 & 4.7 & 8.0 \\
\hline $\mathrm{C} 2$ & VWA & NEW BEETLE & I & $\mathrm{F}$ & L4 & 1.8 & G & M & 32.3 & 5.9 & 11.1 \\
\hline C3 & VWA & JETTA & I & $\mathrm{F}$ & L4 & 1.8 & G & M & 31.3 & 6.1 & 11.4 \\
\hline $\mathrm{C} 4$ & VWA & PASSAT & I & $\mathrm{F}$ & L4 & 1.8 & G & M & 31.3 & 6.1 & 11.4 \\
\hline $\mathrm{C} 8$ & VWA & PASSAT WAGON & I & $\mathrm{F}$ & L4 & 1.8 & G & M & 31.3 & 6.1 & 11.4 \\
\hline \multicolumn{12}{|l|}{ Trucks } \\
\hline T3 & DCC - CHRYSLER & $\begin{array}{l}\text { TOWN \& COUNTRY } \\
\text { 2WD }\end{array}$ & $\mathrm{D}$ & $F$ & V6 & 3.8 & G & $A$ & 23.0 & 8.2 & 15.5 \\
\hline T3 & DCC - CHRYSLER & $\begin{array}{l}\text { TOWN \& COUNTRY } \\
\text { AWD }\end{array}$ & $\mathrm{D}$ & 4 & V6 & 3.8 & G & A & 22.0 & 8.6 & 16.2 \\
\hline Т3 & DCC - DODGE & CARAVAN 2WD & $\mathrm{D}$ & $\mathrm{F}$ & L4 & 2.4 & G & A & 26.0 & 7.3 & 13.7 \\
\hline Т3 & DCC - DODGE & CARAVAN 2WD & $\mathrm{D}$ & $\mathrm{F}$ & V6 & 3.3 & G & A & 24.3 & 7.8 & 14.7 \\
\hline T3 & DCC - DODGE & $\begin{array}{l}\text { GRAND CARAVAN } \\
\text { 2WD }\end{array}$ & $\mathrm{D}$ & $\mathrm{F}$ & V6 & 3.8 & G & A & 23.0 & 8.2 & 15.5 \\
\hline T5 & DCC - MER-BENZ & ML 320 & 1 & 4 & V6 & 3.2 & G & A & 20.8 & 9.1 & 17.2 \\
\hline T5 & DCC - MER-BENZ & ML 430 & I & 4 & V8 & 4.3 & G & A & 19.7 & 9.6 & 18.2 \\
\hline T5 & DCC - MER-BENZ & ML 55 AMG & 1 & 4 & V8 & 5.4 & G & A & 17.7 & 10.7 & 20.2 \\
\hline T3 & DCC - PLYMOUTH & $\begin{array}{l}\text { CARAVAN/VOYAGER } \\
2 W D\end{array}$ & D & $\mathrm{F}$ & V6 & 3.3 & G & A & 24.0 & 7.9 & 14.9 \\
\hline Т3 & DCC - PLYMOUTH & $\begin{array}{l}\text { CARAVAN/VOYAGER } \\
\text { 2WD }\end{array}$ & D & $\mathrm{F}$ & L4 & 2.4 & G & A & 25.9 & 7.3 & 13.8 \\
\hline T1 & FMC - FORD & RANGER 4X2 EV & $D$ & $\mathrm{R}$ & E0 & 2.5 & EL & A & 29.7 & 5.2 & 1.8 \\
\hline T3 & FMC - FORD & WINDSTAR VAN & $\mathrm{D}$ & $\mathrm{F}$ & V6 & 3.8 & G & A & 23.0 & 8.2 & 15.5 \\
\hline T3 & FMC - FORD & WINDSTAR WAGON & $\mathrm{D}$ & $\mathrm{F}$ & V6 & 3.8 & G & A & 22.5 & 8.4 & 15.9 \\
\hline T2 & FMC - FORD & F150 4X2 & $\mathrm{D}$ & $\mathrm{R}$ & V6 & 4.2 & G & M & 21.1 & 9.0 & 16.9 \\
\hline T2 & FMC - FORD & F150 4X2 & $\mathrm{D}$ & $\mathrm{R}$ & V8 & 4.6 & G & A & 20.3 & 9.3 & 17.6 \\
\hline T2 & FMC - FORD & F150 4X2 & $\mathrm{D}$ & $\mathrm{R}$ & V8 & 4.6 & G & M & 19.6 & 9.6 & 18.2 \\
\hline T2 & FMC - FORD & F150 4X2 & $\mathrm{D}$ & $\mathrm{R}$ & V8 & 5.4 & G & A & 19.1 & 9.9 & 18.7 \\
\hline T1 & GMC - CHEVROLET & S10 P/U 2WD & $\mathrm{D}$ & $\mathrm{R}$ & L4 & 2.2 & G & M & 29.6 & 6.5 & 12.1 \\
\hline T3 & GMC - CHEVROLET & VENTURE & $\mathrm{D}$ & $\mathrm{F}$ & V6 & 3.4 & G & A & 25.0 & 7.6 & 14.3 \\
\hline T2 & GMC - CHEVROLET & C1500 SILVERADO & $\mathrm{D}$ & $\mathrm{R}$ & V6 & 4.3 & G & M & 22.5 & 8.4 & 15.9 \\
\hline
\end{tabular}




\begin{tabular}{|c|c|c|c|c|c|c|c|c|c|c|c|}
\hline $\begin{array}{l}\text { Type- } \\
\text { Size } \\
\text { Class }\end{array}$ & Manufacturer/Division & Name & $\begin{array}{l}\text { Dom I } \\
\text { Imp }\end{array}$ & DR & Cyl & Disp & Fuel & Trans & MPG & GHG & Oil Use \\
\hline & & $2 W D$ & & & & & & & & & \\
\hline $\mathrm{T} 2$ & GMC - CHEVROLET & $\begin{array}{l}\text { C1500 SILVERADO } \\
\text { 2WD }\end{array}$ & $\mathrm{D}$ & $\mathrm{R}$ & V8 & 4.8 & G & M & 20.8 & 9.1 & 17.2 \\
\hline T2 & GMC - CHEVROLET & $\begin{array}{l}\text { C1500 SILVERADO } \\
\text { 2WD }\end{array}$ & $\mathrm{D}$ & $\mathrm{R}$ & V8 & 5.3 & G & A & 19.9 & 9.5 & 17.9 \\
\hline $\mathrm{T} 2$ & GMC - CHEVROLET & $\begin{array}{l}\text { C1500 SILVERADO } \\
\text { 2WD }\end{array}$ & D & $\mathrm{R}$ & V8 & 4.8 & G & $A$ & 20.9 & 9.0 & 17.1 \\
\hline T6 & GMC - CHEVROLET & K1500 TAHOE 4WD & $\mathrm{D}$ & 4 & V8 & 4.8 & G & A & 18.3 & 10.3 & 19.5 \\
\hline T6 & GMC - CHEVROLET & C1500 TAHOE 2WD & $\mathrm{D}$ & $\mathrm{R}$ & V8 & 4.8 & G & $A$ & 19.5 & 9.7 & 18.3 \\
\hline T6 & GMC - CHEVROLET & C1500 TAHOE 2WD & $\mathrm{D}$ & $\mathrm{R}$ & V8 & 5.3 & G & A & 18.4 & 10.3 & 19.4 \\
\hline T6 & GMC - CHEVROLET & K1500 TAHOE 4WD & $\mathrm{D}$ & 4 & V8 & 5.3 & G & $A$ & 17.8 & 10.6 & 20.0 \\
\hline T1 & GMC & SONOMA 2WD & I & $\mathrm{R}$ & L4 & 2.2 & G & M & 29.6 & 6.5 & 12.1 \\
\hline $\mathrm{T} 2$ & GMC & C1500 SIERRA 2WD & 1 & $\mathrm{R}$ & V6 & 4.3 & G & M & 22.8 & 8.3 & 15.7 \\
\hline T2 & GMC & C1500 SIERRA 2WD & 1 & $\mathrm{R}$ & V8 & 4.8 & G & M & 20.4 & 9.3 & 17.5 \\
\hline T2 & GMC & C1500 SIERRA 2WD & 1 & $\mathrm{R}$ & V8 & 5.3 & G & A & 20.4 & 9.3 & 17.5 \\
\hline $\mathrm{T} 2$ & GMC & C1500 SIERRA 2WD & 1 & $\mathrm{R}$ & V8 & 4.8 & G & A & 20.9 & 9.0 & 17.1 \\
\hline $\mathrm{T} 2$ & GMC & K1500 SIERRA 4WD & 1 & 4 & V8 & 4.8 & G & M & 19.3 & 9.8 & 18.5 \\
\hline $\mathrm{T} 2$ & GMC & K1500 SIERRA 4WD & 1 & 4 & V8 & 4.8 & G & $A$ & 19.1 & 9.9 & 18.7 \\
\hline T2 & GMC & K1500 SIERRA 4WD & I & 4 & V8 & 5.3 & $\mathrm{G}$ & $A$ & 18.5 & 10.2 & 19.3 \\
\hline T6 & GMC & K1500 YUKON 4WD & 1 & 4 & V8 & 4.8 & G & A & 18.3 & 10.3 & 19.5 \\
\hline T6 & GMC & C1500 YUKON 2WD & I & $\mathrm{R}$ & V8 & 4.8 & G & $A$ & 19.5 & 9.7 & 18.3 \\
\hline T6 & GMC & C1500 YUKON 2WD & 1 & $\mathrm{R}$ & V8 & 5.3 & G & $A$ & 18.4 & 10.3 & 19.4 \\
\hline T6 & GMC & C1500 YUKON 2WD & 1 & $\mathrm{R}$ & V8 & 5.3 & G & $A$ & 18.4 & 10.3 & 19.4 \\
\hline T6 & GMC & K1500 YUKON 4WD & I & 4 & V8 & 5.3 & G & A & 17.8 & 10.6 & 20.0 \\
\hline T3 & GMC - OLDSMOBILE & SILHOUETTE 2WD & $\mathrm{D}$ & $\mathrm{F}$ & V6 & 3.4 & G & A & 25.0 & 7.6 & 14.3 \\
\hline T3 & GMC - PONTIAC & MONTANA FWD & D & $\mathrm{F}$ & V6 & 3.4 & G & A & 25.0 & 7.6 & 14.3 \\
\hline T5 & HONDA & CR-V & 1 & $\mathrm{R}$ & L4 & 2.0 & G & $A$ & 27.5 & 6.9 & 13.0 \\
\hline T5 & HONDA & CR-V & 1 & 4 & L4 & 2.0 & G & A & 27.1 & 7.0 & 13.2 \\
\hline T5 & HONDA & CR-V & 1 & 4 & L4 & 2.0 & G & M & 27.1 & 7.0 & 13.2 \\
\hline T3 & HONDA & ODYSSEY & 1 & $\mathrm{~F}$ & V6 & 3.5 & G & A & 24.0 & 7.9 & 14.9 \\
\hline T5 & MITSUBISHI & $\begin{array}{l}\text { MONTERO } \\
\text { SPORT/NATIVA }\end{array}$ & I & $\mathrm{R}$ & L4 & 2.3 & G & $\mathrm{M}$ & 26.5 & 7.2 & 13.5 \\
\hline T1 & NISSAN & $\begin{array}{l}\text { FRONTIER TRUCK } \\
2 \mathrm{WD}\end{array}$ & I & $\mathrm{R}$ & L4 & 2.4 & G & M & 28.2 & 6.8 & 12.7 \\
\hline T5 & SUBARU & FORESTER & I & 4 & $\mathrm{HO} 4$ & 2.5 & G & $\mathrm{M}$ & 27.9 & 6.8 & 12.8 \\
\hline T5 & SUBARU & FORESTER & 1 & 4 & $\mathrm{HO} 4$ & 2.5 & G & A & 27.9 & 6.8 & 12.8 \\
\hline $\mathrm{T7}$ & TOYOTA - LEXUS & RX 300 2WD & I & $\mathrm{F}$ & V6 & 3.0 & G & A & 24.2 & 7.8 & 14.8 \\
\hline $\mathrm{T} 7$ & TOYOTA - LEXUS & RX 300 4WD & 1 & 4 & V6 & 3.0 & G & $A$ & 23.4 & 8.1 & 15.3 \\
\hline $\mathrm{T} 7$ & TOYOTA & RAV4 2WD & I & $\mathrm{F}$ & L4 & 2.0 & G & M & 30.9 & 6.2 & 11.6 \\
\hline $\mathrm{T7}$ & TOYOTA & RAV4 2WD & I & $\mathrm{F}$ & L4 & 2.0 & G & $A$ & 30.5 & 6.3 & 11.7 \\
\hline T3 & TOYOTA & SIENNA & I & $\mathrm{F}$ & V6 & 3.0 & G & $A$ & 24.0 & 7.9 & 14.9 \\
\hline
\end{tabular}


Energy Star Method 8 Vehicle List

Top $20 \%$ of vehicles by MPG for each body type

\begin{tabular}{|c|c|c|c|c|c|c|c|c|c|c|c|}
\hline $\begin{array}{l}\text { Type- } \\
\text { Size } \\
\text { Class } \\
\end{array}$ & Manufacturer/Division & Name & $\begin{array}{l}\text { Dom I } \\
\text { Imp }\end{array}$ & DR & Cyl & Disp & Fuel & Trans & MPG & GHG & Oil Use \\
\hline \multicolumn{12}{|l|}{ Cars } \\
\hline$\overline{\mathrm{C} 4}$ & DAEWOO & LANOS- 3Dr & $\mathrm{I}$ & $\mathrm{F}$ & L4 & 1.5 & $\mathrm{G}$ & $M$ & 34.8 & 5.5 & 10.3 \\
\hline $\mathrm{C} 4$ & DAEWOO & LANOS-3Dr & I & $\mathrm{F}$ & L4 & 1.6 & G & M & 34.6 & 5.5 & 10.3 \\
\hline $\mathrm{C} 4$ & DAEWOO & LANOS- 3Dr & I & $\mathrm{F}$ & L4 & 1.5 & G & $A$ & 32.9 & 5.8 & 10.8 \\
\hline $\mathrm{C} 4$ & DAEWOO & LANOS-4Dr & 1 & $\mathrm{~F}$ & L4 & 1.6 & G & M & 31.8 & 6.0 & 11.2 \\
\hline $\mathrm{C} 3$ & DCC - CHRYSLER & NEON & D & $\mathrm{F}$ & L4 & 2.0 & $\mathrm{G}$ & M & 36.1 & 5.3 & 9.9 \\
\hline $\mathrm{C} 4$ & DCC - CHRYSLER & CIRRUS & $\mathrm{D}$ & $\mathrm{F}$ & L4 & 2.0 & G & M & 34.3 & 5.6 & 10.4 \\
\hline $\mathrm{C} 3$ & DCC - CHRYSLER & NEON & $\mathrm{D}$ & $\mathrm{F}$ & L4 & 2.0 & G & $A$ & 31.9 & 6.0 & 11.2 \\
\hline $\mathrm{C} 3$ & DCC - DODGE & NEON & $\mathrm{D}$ & $\mathrm{F}$ & L4 & 2.0 & $\mathrm{G}$ & M & 36.1 & 5.3 & 9.9 \\
\hline $\mathrm{C} 3$ & DCC - DODGE & NEON & $\mathrm{D}$ & $\mathrm{F}$ & L4 & 2.0 & G & $A$ & 31.9 & 6.0 & 11.2 \\
\hline $\mathrm{C} 3$ & DCC - PLYMOUTH & NEON & $\mathrm{D}$ & $\mathrm{F}$ & L4 & 2.0 & $\mathrm{G}$ & M & 36.1 & 5.3 & 9.9 \\
\hline $\mathrm{C} 4$ & DCC - PLYMOUTH & BREEZE & $\mathrm{D}$ & $\mathrm{F}$ & L4 & 2.0 & $\mathrm{G}$ & M & 35.4 & 5.4 & 10.1 \\
\hline $\mathrm{C} 3$ & DCC - PLYMOUTH & NEON & $\mathrm{D}$ & $\mathrm{F}$ & L4 & 2.0 & G & $A$ & 31.9 & 6.0 & 11.2 \\
\hline $\mathrm{C} 7$ & FMC - FORD & FOCUS WAGON & $\mathrm{D}$ & $\mathrm{F}$ & L4 & 2.0 & G & M & 36.2 & 5.3 & 9.9 \\
\hline $\mathrm{C} 3$ & FMC - FORD & FOCUS & $D$ & $\mathrm{~F}$ & L4 & 2.0 & G & M & 35.7 & 5.4 & 10.0 \\
\hline $\mathrm{C} 3$ & FMC - FORD & ESCORT & $\mathrm{D}$ & $\mathrm{F}$ & L4 & 2.0 & $\mathrm{G}$ & M & 35.7 & 5.4 & 10.0 \\
\hline $\mathrm{C} 3$ & FMC - FORD & FOCUS & $\mathrm{D}$ & $\mathrm{F}$ & L4 & 2.0 & G & $A$ & 33.9 & 5.7 & 10.5 \\
\hline $\mathrm{C} 7$ & FMC - FORD & FOCUS WAGON & $\mathrm{D}$ & $\mathrm{F}$ & L4 & 2.0 & G & $A$ & 32.6 & 5.9 & 11.0 \\
\hline $\mathrm{C} 3$ & FMC - FORD & ESCORT & $\mathrm{D}$ & $\mathrm{F}$ & L4 & 2.0 & G & $A$ & 33.7 & 5.7 & 10.6 \\
\hline $\mathrm{C} 2$ & FMC - FORD & ESCORT ZX2 & $\mathrm{D}$ & $\mathrm{F}$ & L4 & 2.0 & $\mathrm{G}$ & M & 33.0 & 5.8 & 10.8 \\
\hline $\mathrm{C} 3$ & FMC - FORD & FOCUS 3D & $\mathrm{D}$ & $\mathrm{F}$ & L4 & 2.0 & G & M & 33.0 & 5.8 & 10.8 \\
\hline $\mathrm{C} 3$ & FMC - FORD & FOCUS 3D & $\mathrm{D}$ & $\mathrm{F}$ & L4 & 2.0 & G & $A$ & 32.4 & 5.9 & 11.0 \\
\hline $\mathrm{C} 2$ & FMC - FORD & ESCORT ZX2 & $\mathrm{D}$ & $\mathrm{F}$ & L4 & 2.0 & G & $A$ & 33.1 & 5.8 & 10.8 \\
\hline $\mathrm{C} 7$ & FMC - FORD & FOCUS WAGON & $D$ & $\mathrm{~F}$ & L4 & 2.0 & G & $A$ & 32.6 & 5.9 & 11.0 \\
\hline $\mathrm{C} 3$ & FMC - FORD & CONTOUR & $\mathrm{D}$ & $\mathrm{F}$ & L4 & 2.0 & $\mathrm{G}$ & M & 32.6 & 5.9 & 11.0 \\
\hline $\mathrm{C} 3$ & FMC - MAZDA & PROTEGE & 1 & $\mathrm{~F}$ & L4 & 1.6 & $\mathrm{G}$ & $A$ & 33.8 & 5.7 & 10.6 \\
\hline $\mathrm{C} 4$ & FMC - MAZDA & 626 & I & $\mathrm{F}$ & L4 & 2.0 & G & M & 33.5 & 5.7 & 10.6 \\
\hline $\mathrm{C} 3$ & FMC - MAZDA & PROTEGE & 1 & $\mathrm{~F}$ & L4 & 1.8 & G & M & 32.4 & 5.9 & 11.0 \\
\hline C6 & FMC - MAZDA & MX-5 MIATA & 1 & $\mathrm{R}$ & L4 & 1.8 & G & M & 31.4 & 6.1 & 11.4 \\
\hline $\mathrm{C} 3$ & FMC - MAZDA & PROTEGE & 1 & $\mathrm{~F}$ & L4 & 1.6 & $\mathrm{G}$ & M & 36.5 & 5.3 & 9.8 \\
\hline $\mathrm{C} 3$ & FMC - MERCURY & MYSTIQUE & $\mathrm{D}$ & $\mathrm{F}$ & L4 & 2.0 & G & M & 32.4 & 5.9 & 11.0 \\
\hline $\mathrm{C} 3$ & FMC - MERCURY & COUGAR & $\mathrm{D}$ & $\mathrm{F}$ & L4 & 2.0 & G & M & 32.4 & 5.9 & 11.0 \\
\hline $\mathrm{C} 2$ & GMC - CHEVY & METRO & 1 & $\mathrm{~F}$ & L3 & 1.0 & G & M & 48.9 & 4.0 & 7.3 \\
\hline $\mathrm{C} 2$ & GMC - CHEVY & METRO & 1 & $\mathrm{~F}$ & L4 & 1.3 & $\mathrm{G}$ & M & 45.5 & 4.2 & 7.8 \\
\hline $\mathrm{C} 3$ & GMC - CHEVY & PRIZM & I & $\mathrm{F}$ & L4 & 1.8 & $\mathrm{G}$ & M & 39.6 & 4.9 & 9.0 \\
\hline $\mathrm{C} 3$ & GMC - CHEVY & PRIZM & 1 & $\mathrm{~F}$ & L4 & 1.8 & $\mathrm{G}$ & $A$ & 37.5 & 5.1 & 9.5 \\
\hline $\mathrm{C} 2$ & GMC - CHEVY & METRO & 1 & $\mathrm{~F}$ & L4 & 1.3 & G & $A$ & 37.6 & 5.1 & 9.5 \\
\hline C3 & GMC - CHEVY & PRIZM & 1 & $\mathrm{~F}$ & L4 & 1.8 & G & $A$ & 34.9 & 5.5 & 10.2 \\
\hline $\mathrm{C} 2$ & GMC - CHEVY & CAVALIER & $\mathrm{D}$ & $\mathrm{F}$ & L4 & 2.2 & G & M & 32.1 & 6.0 & 11.1 \\
\hline $\mathrm{C} 2$ & GMC - PONTIAC & SUNFIRE & $\mathrm{D}$ & $\mathrm{F}$ & L4 & 2.2 & G & M & 32.1 & 6.0 & 11.1 \\
\hline $\mathrm{C} 3$ & GMC - SATURN & SL & $\mathrm{D}$ & $\mathrm{F}$ & L4 & 1.9 & G & M & 38.7 & 5.0 & 9.2 \\
\hline $\mathrm{C} 2$ & GMC - SATURN & SC & $\mathrm{D}$ & $\mathrm{F}$ & L4 & 1.9 & G & $\mathrm{M}$ & 35.4 & 5.4 & 10.1 \\
\hline $\mathrm{C} 2$ & GMC - SATURN & SC & $\mathrm{D}$ & $\mathrm{F}$ & L4 & 1.9 & G & M & 38.1 & 5.0 & 9.4 \\
\hline C3 & GMC - SATURN & SL & $\mathrm{D}$ & $\mathrm{F}$ & L4 & 1.9 & $\mathrm{G}$ & M & 36.1 & 5.3 & 9.9 \\
\hline
\end{tabular}




\begin{tabular}{|c|c|c|c|c|c|c|c|c|c|c|c|c|}
\hline $\begin{array}{l}\text { Type- } \\
\text { Size } \\
\text { Class }\end{array}$ & Manufacturer/Division & Name & $\begin{array}{l}\text { Dom I } \\
\text { Imp }\end{array}$ & DR & Cyl & Disp & Fuel & Trans & MPG & GHG & \multicolumn{2}{|c|}{ Oil Use } \\
\hline$\overline{C 7}$ & GMC - SATURN & SW & $\mathrm{D}$ & $\mathrm{F}$ & L4 & 1.9 & $\mathrm{G}$ & $\mathrm{M}$ & 36.1 & & 5.3 & 9.9 \\
\hline C3 & GMC - SATURN & SL & $\mathrm{D}$ & $\mathrm{F}$ & L4 & 1.9 & G & $A$ & 34.4 & & 5.6 & 10.4 \\
\hline $\mathrm{C} 2$ & GMC - SATURN & SC & $\mathrm{D}$ & $\mathrm{F}$ & L4 & 1.9 & G & $A$ & 33.9 & & 5.7 & 10.5 \\
\hline $\mathrm{C7}$ & GMC - SATURN & SW & $\mathrm{D}$ & $\mathrm{F}$ & L4 & 1.9 & G & $A$ & 35.4 & & 5.4 & 10.1 \\
\hline C2 & GMC - SATURN & SC & $\mathrm{D}$ & $\mathrm{F}$ & L4 & 1.9 & G & A & 36.1 & & 5.3 & 9.9 \\
\hline C3 & GMC - SATURN & SL & $\mathrm{D}$ & $\mathrm{F}$ & L4 & 1.9 & G & $A$ & 36.0 & & 5.3 & 9.9 \\
\hline $\mathrm{C} 7$ & GMC - SATURN & SW & $\mathrm{D}$ & $\mathrm{F}$ & L4 & 1.9 & G & A & 33.9 & & 5.7 & 10.5 \\
\hline C4 & GMC - SATURN & LS & $\mathrm{D}$ & $\mathrm{F}$ & L4 & 2.2 & G & M & 31.9 & & 6.0 & 11.2 \\
\hline $\mathrm{C} 2$ & HONDA - ACURA & ACURA INTEGRA & I & $\mathrm{F}$ & L4 & 1.8 & G & M & 32.3 & & 5.9 & 11.0 \\
\hline C2 & HONDA - ACURA & ACURA INTEGRA -R & I & $\mathrm{F}$ & L4 & 1.8 & G & M & 31.9 & & 6.0 & 11.2 \\
\hline C6 & HONDA & INSIGHT & I & $\mathrm{F}$ & L3 & 1.0 & $\mathrm{G} / \mathrm{EL}$ & M & 76.3 & & 2.6 & 4.7 \\
\hline C2 & HONDA & CIVIC HX & I & $\mathrm{F}$ & L4 & 1.6 & G & M & 44.7 & & 4.3 & 8.0 \\
\hline C2 & HONDA & CIVIC HX & I & $\mathrm{F}$ & L4 & 1.6 & G & A & 41.9 & & 4.6 & 8.5 \\
\hline C2 & HONDA & CIVIC dx 4Dr & I & $\mathrm{F}$ & L4 & 1.6 & G & M & 37.2 & & 5.2 & 9.6 \\
\hline C2 & HONDA & CIVIC ex & I & $\mathrm{F}$ & L4 & 1.6 & G & M & 37.2 & & 5.2 & 9.6 \\
\hline C2 & HONDA & CIVIC vp & I & $\mathrm{F}$ & L4 & 1.6 & G & $A$ & 35.5 & & 5.4 & 10.1 \\
\hline C2 & HONDA & CIVIC dx 4Dr & I & $\mathrm{F}$ & L4 & 1.6 & G & A & 35.6 & & 5.4 & 10.0 \\
\hline C2 & HONDA & CIVIC ex & I & $\mathrm{F}$ & L4 & 1.6 & G & A & 35.5 & & 5.4 & 10.1 \\
\hline C2 & HONDA & CIVIC si & I & $\mathrm{F}$ & L4 & 1.6 & G & $\mathrm{M}$ & 32.8 & & 5.8 & 10.9 \\
\hline C4 & HONDA & ACCORD Ix & I & $\mathrm{F}$ & L4 & 2.3 & G & $\mathrm{M}$ & 32.1 & & 6.0 & 11.1 \\
\hline C3 & HYUNDAI & ACCENT & I & $\mathrm{F}$ & L4 & 1.5 & G & $\mathrm{M}$ & 36.6 & & 5.3 & 9.8 \\
\hline C3 & HYUNDAI & ACCENT & I & $\mathrm{F}$ & L4 & 1.5 & G & A & 34.2 & & 5.6 & 10.4 \\
\hline C3 & HYUNDAI & ELANTRA & I & $\mathrm{F}$ & L4 & 2.0 & G & M & 32.1 & & 6.0 & 11.1 \\
\hline C2 & MITSUBISHI & MIRAGE 2D & I & $\mathrm{F}$ & L4 & 1.5 & G & $\mathrm{M}$ & 41.7 & & 4.6 & 8.6 \\
\hline C2 & MITSUBISHI & MIRAGE & I & $\mathrm{F}$ & L4 & 1.8 & G & M & 36.3 & & 5.3 & 9.8 \\
\hline C2 & MITSUBISHI & MIRAGE 2D & I & $\mathrm{F}$ & L4 & 1.5 & G & $A$ & 36.7 & & 5.2 & 9.7 \\
\hline C2 & MITSUBISHI & MIRAGE & I & $\mathrm{F}$ & L4 & 1.8 & G & A & 33.2 & & 5.8 & 10.8 \\
\hline C3 & NISSAN & SENTRA & I & $\mathrm{F}$ & L4 & 1.8 & G & M & 34.9 & & 5.5 & 10.2 \\
\hline C3 & NISSAN & SENTRA & I & $\mathrm{F}$ & L4 & 1.8 & G & A & 33.9 & & 5.6 & 10.5 \\
\hline C2 & SUZUKI & SWIFT & I & $\mathrm{F}$ & L4 & 1.3 & G & $\mathrm{M}$ & 45.5 & & 4.2 & 7.8 \\
\hline C2 & SUZUKI & ESTEEM & I & $\mathrm{F}$ & L4 & 1.6 & G & $\mathrm{M}$ & 38.6 & & 5.0 & 9.3 \\
\hline C2 & SUZUKI & SWIFT & I & $\mathrm{F}$ & L4 & 1.3 & G & A & 37.6 & & 5.1 & 9.5 \\
\hline C2 & SUZUKI & ESTEEM & I & $\mathrm{F}$ & L4 & 1.8 & G & M & 36.2 & & 5.3 & 9.9 \\
\hline C7 & SUZUKI & ESTEEM WAGON & I & $\mathrm{F}$ & L4 & 1.8 & G & M & 35.1 & & 5.5 & 10.2 \\
\hline C2 & SUZUKI & ESTEEM & I & $\mathrm{F}$ & L4 & 1.6 & G & A & 35.0 & & 5.5 & 10.2 \\
\hline $\mathrm{C} 7$ & SUZUKI & ESTEEM WAGON & I & $\mathrm{F}$ & L4 & 1.6 & G & $A$ & 33.7 & & 5.7 & 10.6 \\
\hline C7 & SUZUKI & ESTEEM WAGON & I & $\mathrm{F}$ & L4 & 1.8 & G & A & 33.7 & & 5.7 & 10.6 \\
\hline C2 & SUZUKI & ESTEEM & I & $\mathrm{F}$ & L4 & 1.8 & G & A & 33.7 & & 5.7 & 10.6 \\
\hline C3 & TOYOTA & $\mathrm{ECHO}$ & I & $\mathrm{F}$ & L4 & 1.5 & G & M & 43.1 & & 4.5 & 8.3 \\
\hline C3 & TOYOTA & COROLLA & I & $\mathrm{F}$ & L4 & 1.8 & G & M & 39.6 & & 4.9 & 9.0 \\
\hline C3 & TOYOTA & $\mathrm{ECHO}$ & I & $\mathrm{F}$ & L4 & 1.5 & G & A & 39.4 & & 4.9 & 9.1 \\
\hline C3 & TOYOTA & COROLLA & I & $\mathrm{F}$ & L4 & 1.8 & G & A & 37.4 & & 5.1 & 9.6 \\
\hline C2 & TOYOTA & CELICA & I & $\mathrm{F}$ & L4 & 1.8 & G & $\mathrm{M}$ & 35.2 & & 5.4 & 10.1 \\
\hline C2 & TOYOTA & CELICA & I & $\mathrm{F}$ & L4 & 1.8 & G & A & 34.9 & & 5.5 & 10.2 \\
\hline C3 & TOYOTA & COROLLA & I & $\mathrm{F}$ & L4 & 1.8 & G & A & 34.9 & & 5.5 & 10.2 \\
\hline C6 & TOYOTA & MR2 & I & $F$ & L4 & 1.8 & G & $\mathrm{M}$ & 31.8 & & 6.0 & 11.2 \\
\hline C3 & VWA - AUDI & AUDI A4 & I & $\mathrm{F}$ & L4 & 1.8 & G & $\mathrm{M}$ & 31.9 & & 6.0 & 11.2 \\
\hline
\end{tabular}




\begin{tabular}{|c|c|c|c|c|c|c|c|c|c|c|c|c|}
\hline $\begin{array}{l}\text { Type- } \\
\text { Size } \\
\text { Class }\end{array}$ & Manufacturer/Division & Name & $\begin{array}{l}\text { Dom I } \\
\text { Imp }\end{array}$ & DR & Cyl & Disp $F$ & Fuel & Trans & MPG & GHG & \multicolumn{2}{|c|}{ Oil Use } \\
\hline$\overline{\mathrm{C} 2}$ & VWA & NEW BEETLE & $T$ & $\bar{F}$ & L4 & 1.9 & $\mathrm{D}$ & $\mathrm{M}$ & 52.7 & & 4.0 & 6.8 \\
\hline $\mathrm{C} 3$ & VWA & GOLF TDI & I & $\mathrm{F}$ & L4 & 1.9 & $D$ & M & 52.7 & & 4.0 & 6.8 \\
\hline C3 & VWA & JETTA & 1 & $\mathrm{~F}$ & L4 & 1.9 & $\mathrm{D}$ & M & 52.7 & & 4.0 & 6.8 \\
\hline $\mathrm{C} 2$ & VWA & NEW BEETLE & I & $\mathrm{F}$ & L4 & 1.9 & $D$ & $A$ & 44.7 & & 4.7 & 8.0 \\
\hline C3 & VWA & GOLF TDI & I & $\mathrm{F}$ & L4 & 1.9 & $\mathrm{D}$ & $A$ & 44.7 & & 4.7 & 8.0 \\
\hline C3 & VWA & JETTA & I & $\mathrm{F}$ & L4 & 1.9 & $D$ & $A$ & 44.7 & & 4.7 & 8.0 \\
\hline $\mathrm{C} 2$ & VWA & NEW BEETLE & I & $\mathrm{F}$ & L4 & 1.8 & G & $M$ & 32.3 & & 5.9 & 11.1 \\
\hline C3 & VWA & GTI & 1 & $\mathrm{~F}$ & L4 & 1.8 & G & M & 31.3 & & 6.1 & 11.4 \\
\hline C3 & VWA & GOLF & I & $\mathrm{F}$ & L4 & 1.8 & G & $M$ & 31.3 & & 6.1 & 11.4 \\
\hline C3 & VWA & JETTA & I & $\mathrm{F}$ & L4 & 1.8 & $G$ & M & 31.3 & & 6.1 & 11.4 \\
\hline \multicolumn{13}{|l|}{ Trucks } \\
\hline T3 & DCC - DODGE & CARAVAN 2WD & $\mathrm{D}$ & $\mathrm{F}$ & L4 & 2.4 & $\mathrm{G}$ & $A$ & 26.0 & & 7.3 & 13.7 \\
\hline T1 & DCC - DODGE & DAKOTA 2WD & D & $\mathrm{R}$ & L4 & 2.5 & $\mathrm{G}$ & $M$ & 25.7 & & 7.4 & 13.9 \\
\hline T3 & DCC - DODGE & CARAVAN 2WD & D & $\mathrm{F}$ & V6 & 3.3 & G & $A$ & 24.3 & & 7.8 & 14.7 \\
\hline T3 & DCC - DODGE & CARAVAN 2WD & D & $F$ & V6 & 3.0 & G & $A$ & 24.0 & & 7.9 & 14.9 \\
\hline T5 & DCC - JEEP & CHEROKEE 2WD & I & $\mathrm{R}$ & L4 & 2.5 & $G$ & M & 25.7 & & 7.4 & 13.9 \\
\hline T3 & DCC - PLYMOUTH & $\begin{array}{l}\text { CARAVAN/VOYAGER } \\
\text { 2WD }\end{array}$ & $\mathrm{D}$ & $\mathrm{F}$ & V6 & 3.3 & G & $A$ & 24.0 & & 7.9 & 14.9 \\
\hline T3 & DCC - PLYMOUTH & $\begin{array}{l}\text { CARAVAN/VOYAGER } \\
\text { 2WD }\end{array}$ & $\mathrm{D}$ & $\mathrm{F}$ & L4 & 2.4 & G & $A$ & 25.9 & & 7.3 & 13.8 \\
\hline T3 & DCC - PLYMOUTH & $\begin{array}{l}\text { CARAVAN/VOYAGER } \\
\text { 2WD }\end{array}$ & $\mathrm{D}$ & $\mathrm{F}$ & V6 & 3.0 & G & $A$ & 24.0 & & 7.9 & 14.9 \\
\hline T1 & FMC - FORD & RANGER 4X2 EV & D & $\mathrm{R}$ & E0 & 2.5 & EL & $A$ & 29.7 & & 5.2 & 1.8 \\
\hline T1 & FMC - FORD & RANGER 4X2 & $\mathrm{D}$ & $\mathrm{R}$ & L4 & 2.5 & G & M & 28.3 & & 6.7 & 12.6 \\
\hline T1 & FMC - FORD & RANGER 4X2 & D & $\mathrm{R}$ & L4 & 2.5 & G & $A$ & 25.5 & & 7.5 & 14.0 \\
\hline T1 & GMC - CHEVROLET & S10 P/U 2WD & D & $\mathrm{R}$ & L4 & 2.2 & $\mathrm{FF}$ & M & 23.9 & & 5.9 & 7.6 \\
\hline $\mathrm{T} 7$ & GMC - CHEVROLET & TRACKER CONV 2WD & D & $\mathrm{R}$ & L4 & 1.6 & G & M & 31.1 & & 6.2 & 11.5 \\
\hline $\mathrm{T} 7$ & GMC - CHEVROLET & TRACKER CONV 4 X4 & D & 4 & L4 & 1.6 & G & M & 30.7 & & 6.2 & 11.6 \\
\hline T1 & GMC - CHEVROLET & S10 P/U 2WD & $\mathrm{D}$ & $\mathrm{R}$ & L4 & 2.2 & G & M & 29.6 & & 6.5 & 12.1 \\
\hline $\mathrm{T7}$ & GMC - CHEVROLET & TRACKER CONV 2WD & $\mathrm{D}$ & $\mathrm{R}$ & L4 & 2.0 & G & $A$ & 29.1 & & 6.6 & 12.3 \\
\hline $\mathrm{T} 7$ & GMC - CHEVROLET & TRACKER VAN 2WD & D & $\mathrm{R}$ & L4 & 2.0 & G & $A$ & 28.5 & & 6.7 & 12.5 \\
\hline $\mathrm{T} 7$ & GMC - CHEVROLET & TRACKER CONV 2WD & D & $\mathrm{R}$ & L4 & 2.0 & G & M & 28.0 & & 6.8 & 12.8 \\
\hline $\mathrm{T7}$ & GMC - CHEVROLET & TRACKER VAN 2WD & D & $\mathrm{R}$ & L4 & 2.0 & G & $M$ & 28.0 & & 6.8 & 12.7 \\
\hline T7 & GMC - CHEVROLET & TRACKER CONV 4X4 & $\mathrm{D}$ & 4 & L4 & 2.0 & G & A & 27.9 & & 6.8 & 12.8 \\
\hline T7 & GMC - CHEVROLET & TRACKER CONV 4 X4 & $\mathrm{D}$ & 4 & L4 & 2.0 & G & M & 27.5 & & 6.9 & 13.0 \\
\hline $\mathrm{T} 7$ & GMC - CHEVROLET & TRACKER VAN 4X4 & D & 4 & L4 & 2.0 & G & $A$ & 27.9 & & 6.8 & 12.8 \\
\hline $\mathrm{T} 7$ & GMC - CHEVROLET & TRACKER VAN 4X4 & D & 4 & L4 & 2.0 & G & M & 27.4 & & 7.0 & 13.0 \\
\hline T1 & GMC - CHEVROLET & S10 P/U 2WD & D & $\mathrm{R}$ & L4 & 2.2 & G & $A$ & 25.2 & & 7.5 & 14.2 \\
\hline T3 & GMC - CHEVROLET & VENTURE & D & $\mathrm{F}$ & V6 & 3.4 & G & $A$ & 25.0 & & 7.6 & 14.3 \\
\hline T1 & GMC & SONOMA 2WD & I & $\mathrm{R}$ & L4 & 2.2 & $\mathrm{FF}$ & M & 23.9 & & 5.9 & 7.6 \\
\hline T1 & GMC & SONOMA 2WD & I & $\mathrm{R}$ & L4 & 2.2 & G & M & 29.6 & & 6.5 & 12.1 \\
\hline T1 & GMC & SONOMA 2WD & I & $\mathrm{R}$ & L4 & 2.2 & G & $A$ & 24.6 & & 7.7 & 14.5 \\
\hline T3 & GMC - OLDSMOBILE & SILHOUETTE 2WD & $\mathrm{D}$ & $\mathrm{F}$ & V6 & 3.4 & G & $A$ & 25.0 & & 7.6 & 14.3 \\
\hline T3 & GMC - PONTIAC & MONTANA FWD & $\mathrm{D}$ & $\mathrm{F}$ & V6 & 3.4 & G & A & 25.0 & & 7.6 & 14.3 \\
\hline T5 & HONDA & CR-V & I & $\mathrm{R}$ & L4 & 2.0 & G & $A$ & 27.5 & & 6.9 & 13.0 \\
\hline T5 & HONDA & CR-V & I & 4 & L4 & 2.0 & G & $A$ & 27.1 & & 7.0 & 13.2 \\
\hline T5 & HONDA & CR-V & I & 4 & L4 & 2.0 & G & $\mathrm{M}$ & 27.1 & & 7.0 & 13.2 \\
\hline T3 & HONDA & ODYSSEY & I & $F$ & V6 & 3.5 & G & $A$ & 24.0 & & 7.9 & 14.9 \\
\hline
\end{tabular}




\begin{tabular}{|c|c|c|c|c|c|c|c|c|c|c|c|c|}
\hline $\begin{array}{l}\text { Type- } \\
\text { Size } \\
\text { Class } \\
\end{array}$ & Manufacturer/Division & Name & $\begin{array}{l}\text { Dom I } \\
\text { Imp }\end{array}$ & DR & Cyl & Disp & Fuel & Trans & MPG & GHG & & Use \\
\hline$\overline{\mathrm{T} 1}$ & ISUZU & HOMBRE P/U 2WD & $T$ & $\mathrm{R}$ & L4 & 2.2 & $\mathrm{FF}$ & $\bar{M}$ & 23.9 & & 5.9 & 7.6 \\
\hline $\mathrm{T} 1$ & ISUZU & HOMBRE P/U 2WD & 1 & $\mathrm{R}$ & L4 & 2.2 & G & M & 28.4 & & 6.7 & 12.6 \\
\hline T5 & ISUZU & RODEO 2WD & 1 & $\mathrm{R}$ & L4 & 2.2 & G & M & 24.9 & & 7.6 & 14.4 \\
\hline T5 & ISUZU & AMIGO 2WD & 1 & $\mathrm{R}$ & L4 & 2.2 & G & M & 24.8 & & 7.6 & 14.4 \\
\hline T1 & ISUZU & HOMBRE P/U 2WD & I & $\mathrm{R}$ & L4 & 2.2 & G & A & 24.6 & & 7.7 & 14.5 \\
\hline $\mathrm{T7}$ & $\mathrm{KIA}$ & SPORTAGE & I & $\mathrm{R}$ & L4 & 2.0 & G & M & 24.9 & & 7.6 & 14.3 \\
\hline $\mathrm{T} 7$ & $\mathrm{KIA}$ & SPORTAGE & 1 & 4 & L4 & 2.0 & G & M & 24.6 & & 7.7 & 14.5 \\
\hline T5 & MITSUBISHI & $\begin{array}{l}\text { MONTERO } \\
\text { SPORT/NATIVA }\end{array}$ & I & $\mathrm{R}$ & L4 & 2.3 & G & M & 26.5 & & 7.2 & 13.5 \\
\hline T5 & MITSUBISHI & $\begin{array}{l}\text { MONTERO } \\
\text { SPORT/NATIVA }\end{array}$ & 1 & $\mathrm{R}$ & L4 & 2.3 & G & A & 24.2 & & 7.8 & 14.7 \\
\hline T1 & NISSAN & $\begin{array}{l}\text { FRONTIER TRUCK } \\
2 \mathrm{WD}\end{array}$ & 1 & $\mathrm{R}$ & L4 & 2.4 & G & M & 28.2 & & 6.8 & 12.7 \\
\hline $\mathrm{T} 1$ & NISSAN & $\begin{array}{l}\text { FRONTIER TRUCK } \\
\text { 2WD }\end{array}$ & I & $\mathrm{R}$ & L4 & 2.4 & G & A & 25.0 & & 7.6 & 14.3 \\
\hline T5 & NISSAN & XTERRA 2WD & 1 & $\mathrm{R}$ & L4 & 2.4 & G & M & 24.2 & & 7.9 & 14.8 \\
\hline T5 & SUBARU & FORESTER & 1 & 4 & $\mathrm{HO} 4$ & 2.5 & G & M & 27.9 & & 6.8 & 12.8 \\
\hline T5 & SUBARU & FORESTER & I & 4 & $\mathrm{HO} 4$ & 2.5 & G & A & 27.9 & & 6.8 & 12.8 \\
\hline $\mathrm{T} 7$ & SUZUKI & VITARA CONV 2WD & 1 & $\mathrm{R}$ & L4 & 1.6 & G & M & 31.1 & & 6.1 & 11.5 \\
\hline $\mathrm{T} 7$ & SUZUKI & VITARA CONV 4WD & 1 & 4 & L4 & 1.6 & G & M & 30.7 & & 6.2 & 11.6 \\
\hline T7 & SUZUKI & VITARA CONV 2WD & 1 & $\mathrm{R}$ & L4 & 2.0 & G & A & 27.8 & & 6.8 & 12.8 \\
\hline $\mathrm{T} 7$ & SUZUKI & VITARA VAN 2WD & I & $\mathrm{R}$ & L4 & 2.0 & G & A & 27.7 & & 6.9 & 12.9 \\
\hline $\mathrm{T} 7$ & SUZUKI & VITARA CONV 2WD & I & $\mathrm{R}$ & L4 & 2.0 & G & M & 27.2 & & 7.0 & 13.1 \\
\hline T7 & SUZUKI & VITARA VAN 2WD & I & $\mathrm{R}$ & L4 & 2.0 & G & M & 27.3 & & 7.0 & 13.1 \\
\hline $\mathrm{T} 7$ & SUZUKI & VITARA CONV 4WD & 1 & 4 & L4 & 2.0 & G & A & 27.8 & & 6.8 & 12.8 \\
\hline $\mathrm{T} 7$ & SUZUKI & VITARA CONV 4WD & I & 4 & L4 & 2.0 & G & M & 27.2 & & 7.0 & 13.1 \\
\hline $\mathrm{T} 7$ & SUZUKI & VITARA VAN 4WD & 1 & 4 & L4 & 2.0 & G & A & 27.7 & & 6.9 & 12.9 \\
\hline $\mathrm{T} 7$ & SUZUKI & VITARA VAN 4WD & I & 4 & L4 & 2.0 & G & M & 27.3 & & 7.0 & 13.1 \\
\hline $\mathrm{T} 7$ & SUZUKI & GRAND VITARA & 1 & $\mathrm{R}$ & V6 & 2.5 & G & M & 24.0 & & 7.9 & 14.9 \\
\hline T1 & TKM - MAZDA & MAZDA 4X2 & 1 & $\mathrm{R}$ & L4 & 2.5 & G & M & 28.3 & & 6.7 & 12.6 \\
\hline T1 & TKM - MAZDA & MAZDA 4X2 & I & $\mathrm{R}$ & L4 & 2.5 & G & A & 25.5 & & 7.5 & 14.0 \\
\hline $\mathrm{T} 7$ & TOYOTA - LEXUS & RX $3002 W D$ & 1 & $\mathrm{~F}$ & V6 & 3.0 & G & A & 24.2 & & 7.8 & 14.8 \\
\hline T7 & TOYOTA & RAV4 2WD & I & $\mathrm{F}$ & L4 & 2.0 & G & M & 30.9 & & 6.2 & 11.6 \\
\hline T7 & TOYOTA & RAV4 2WD & 1 & $\mathrm{~F}$ & L4 & 2.0 & G & A & 30.5 & & 6.3 & 11.7 \\
\hline $\mathrm{T} 7$ & TOYOTA & RAV4 4WD & 1 & 4 & L4 & 2.0 & G & A & 27.8 & & 6.9 & 12.8 \\
\hline $\mathrm{T} 7$ & TOYOTA & RAV4 4WD & 1 & 4 & L4 & 2.0 & G & M & 26.9 & & 7.1 & 13.3 \\
\hline T1 & TOYOTA & $\begin{array}{l}\text { TOYOTA TACOMA } \\
\text { 2WD }\end{array}$ & 1 & $\mathrm{R}$ & L4 & 2.4 & G & M & 27.4 & & 7.0 & 13.1 \\
\hline T1 & TOYOTA & $\begin{array}{l}\text { TOYOTA TACOMA } \\
\text { 2WD }\end{array}$ & 1 & $\mathrm{R}$ & L4 & 2.4 & G & A & 26.2 & & 7.2 & 13.6 \\
\hline T5 & TOYOTA & 4RUNNER 2WD & 1 & $\mathrm{R}$ & L4 & 2.7 & G & A & 25.0 & & 7.6 & 14.3 \\
\hline $\mathrm{T} 1$ & TOYOTA & $\begin{array}{l}\text { TOYOTA TACOMA } \\
\text { 2WD }\end{array}$ & 1 & $\mathrm{R}$ & V6 & 3.4 & G & M & 24.3 & & 7.8 & 14.7 \\
\hline T3 & TOYOTA & SIENNA & I & $\mathrm{F}$ & V6 & 3.0 & G & A & 24.0 & & 7.9 & 14.9 \\
\hline
\end{tabular}


Energy Star Method 9 Vehicle List

Top $20 \%$ of vehicles by MPG in each type-size class

\begin{tabular}{|c|c|c|c|c|c|c|c|c|c|c|c|}
\hline $\begin{array}{l}\text { Type- } \\
\text { Size } \\
\text { Class }\end{array}$ & Manufacturer/Division & Name & $\begin{array}{l}\text { Dom I } \\
\text { Imp }\end{array}$ & DR & Cyl & Disp $F$ & Fuel & Trans & IPG & GHG & $\begin{array}{l}\text { Oil } \\
\text { Use }\end{array}$ \\
\hline \multicolumn{12}{|c|}{ Minicompact Car } \\
\hline$\overline{C 1}$ & DCC - MER-BENZ & CLK 320 (CABRIOLET) & 1 & $\mathrm{R}$ & V6 & 3.2 & G & $A$ & 26.5 & 7.2 & 13.5 \\
\hline C1 & PORSCHE - PORSCH & 911 CARRERA & I & $\mathrm{R}$ & $\mathrm{H} 6$ & 3.4 & G & $M$ & 23.2 & 8.2 & 15.4 \\
\hline C1 & VWA - AUDI & AUDI TT COUPE & 1 & 4 & L4 & 1.8 & G & $M$ & 29.4 & 6.5 & 12.1 \\
\hline \multicolumn{12}{|c|}{ Subcompact Car } \\
\hline $\mathrm{C} 2$ & GMC - CHEVY & METRO & 1 & $\mathrm{~F}$ & L3 & 1.0 & G & $M$ & 48.9 & 4.0 & 7.3 \\
\hline $\mathrm{C} 2$ & GMC - CHEVY & METRO & 1 & $\mathrm{~F}$ & L4 & 1.3 & G & M & 45.5 & 4.2 & 7.8 \\
\hline $\mathrm{C} 2$ & GMC - CHEVY & METRO & 1 & $\mathrm{~F}$ & L4 & 1.3 & G & A & 37.6 & 5.1 & 9.5 \\
\hline $\mathrm{C} 2$ & GMC - SATURN & SC & $\mathrm{D}$ & $\mathrm{F}$ & L4 & 1.9 & G & M & 38.1 & 5.0 & 9.4 \\
\hline $\mathrm{C} 2$ & GMC - SATURN & SC & D & $\mathrm{F}$ & L4 & 1.9 & G & A & 36.1 & 5.3 & 9.9 \\
\hline $\mathrm{C} 2$ & HONDA & CIVIC HX & 1 & $\mathrm{~F}$ & L4 & 1.6 & G & M & 44.7 & 4.3 & 8.0 \\
\hline $\mathrm{C} 2$ & HONDA & CIVIC HX & 1 & $\mathrm{~F}$ & L4 & 1.6 & G & A & 41.9 & 4.6 & 8.5 \\
\hline $\mathrm{C} 2$ & HONDA & CIVIC dx 4Dr & 1 & $\mathrm{~F}$ & L4 & 1.6 & G & $M$ & 37.2 & 5.2 & 9.6 \\
\hline $\mathrm{C} 2$ & HONDA & CIVIC ex & 1 & $\mathrm{~F}$ & L4 & 1.6 & G & $M$ & 37.2 & 5.2 & 9.6 \\
\hline $\mathrm{C} 2$ & HONDA & CIVIC vp & 1 & $\mathrm{~F}$ & L4 & 1.6 & G & $A$ & 35.5 & 5.4 & 10.1 \\
\hline $\mathrm{C} 2$ & HONDA & CIVIC dx 4Dr & 1 & $\mathrm{~F}$ & L4 & 1.6 & G & A & 35.6 & 5.4 & 10.0 \\
\hline $\mathrm{C} 2$ & HONDA & CIVIC ex & 1 & $\mathrm{~F}$ & L4 & 1.6 & G & A & 35.5 & 5.4 & 10.1 \\
\hline $\mathrm{C} 2$ & MITSUBISHI & MIRAGE 2D & 1 & $\mathrm{~F}$ & L4 & 1.5 & G & M & 41.7 & 4.6 & 8.6 \\
\hline $\mathrm{C} 2$ & MITSUBISHI & MIRAGE & I & $\mathrm{F}$ & L4 & 1.8 & G & M & 36.3 & 5.3 & 9.8 \\
\hline $\mathrm{C} 2$ & MITSUBISHI & MIRAGE 2D & 1 & $\mathrm{~F}$ & L4 & 1.5 & G & A & 36.7 & 5.2 & 9.7 \\
\hline $\mathrm{C} 2$ & SUZUKI & SWIFT & 1 & $\mathrm{~F}$ & L4 & 1.3 & G & M & 45.5 & 4.2 & 7.8 \\
\hline $\mathrm{C} 2$ & SUZUKI & ESTEEM & 1 & $\mathrm{~F}$ & L4 & 1.6 & G & M & 38.6 & 5.0 & 9.3 \\
\hline $\mathrm{C} 2$ & SUZUKI & SWIFT & 1 & $\mathrm{~F}$ & L4 & 1.3 & G & A & 37.6 & 5.1 & 9.5 \\
\hline $\mathrm{C} 2$ & SUZUKI & ESTEEM & 1 & $\mathrm{~F}$ & L4 & 1.8 & G & M & 36.2 & 5.3 & 9.9 \\
\hline $\mathrm{C} 2$ & VWA & NEW BEETLE & 1 & $\mathrm{~F}$ & L4 & 1.9 & $\mathrm{D}$ & M & 52.7 & 4.0 & 6.8 \\
\hline $\mathrm{C} 2$ & VWA & NEW BEETLE & I & $\mathrm{F}$ & L4 & 1.9 & $\mathrm{D}$ & A & 44.7 & 4.7 & 8.0 \\
\hline \multicolumn{12}{|c|}{ Compact Car } \\
\hline C3 & DCC - CHRYSLER & NEON & $\mathrm{D}$ & $\mathrm{F}$ & L4 & 2.0 & G & $M$ & 36.1 & 5.3 & 9.9 \\
\hline C3 & DCC - DODGE & NEON & D & $\mathrm{F}$ & L4 & 2.0 & G & M & 36.1 & 5.3 & 9.9 \\
\hline C3 & DCC - PLYMOUTH & NEON & D & $\mathrm{F}$ & L4 & 2.0 & G & M & 36.1 & 5.3 & 9.9 \\
\hline C3 & FMC - FORD & FOCUS & $\mathrm{D}$ & $\mathrm{F}$ & L4 & 2.0 & G & M & 35.7 & 5.4 & 10.0 \\
\hline C3 & FMC - FORD & ESCORT & D & $\mathrm{F}$ & L4 & 2.0 & G & M & 35.7 & 5.4 & 10.0 \\
\hline C3 & FMC - MAZDA & PROTEGE & 1 & $\mathrm{~F}$ & L4 & 1.6 & G & M & 36.5 & 5.3 & 9.8 \\
\hline C3 & GMC - CHEVY & PRIZM & 1 & $\mathrm{~F}$ & L4 & 1.8 & G & M & 39.6 & 4.9 & 9.0 \\
\hline C3 & GMC - CHEVY & PRIZM & 1 & $\mathrm{~F}$ & L4 & 1.8 & G & A & 37.5 & 5.1 & 9.5 \\
\hline C3 & GMC - CHEVY & PRIZM & 1 & $\mathrm{~F}$ & L4 & 1.8 & G & A & 34.9 & 5.5 & 10.2 \\
\hline C3 & GMC - SATURN & SL & $\mathrm{D}$ & $\mathrm{F}$ & L4 & 1.9 & G & M & 38.7 & 5.0 & 9.2 \\
\hline C3 & GMC - SATURN & SL & $\mathrm{D}$ & $\mathrm{F}$ & L4 & 1.9 & G & M & 36.1 & 5.3 & 9.9 \\
\hline C3 & GMC - SATURN & SL & D & $\mathrm{F}$ & L4 & 1.9 & G & A & 34.4 & 5.6 & 10.4 \\
\hline C3 & GMC - SATURN & SL & D & $\mathrm{F}$ & L4 & 1.9 & G & A & 36.0 & 5.3 & 9.9 \\
\hline C3 & HYUNDAI & ACCENT & 1 & $\mathrm{~F}$ & L4 & 1.5 & G & M & 36.6 & 5.3 & 9.8 \\
\hline C3 & HYUNDAI & ACCENT & 1 & $\mathrm{~F}$ & L4 & 1.5 & G & A & 34.2 & 5.6 & 10.4 \\
\hline C3 & NISSAN & SENTRA & 1 & $\mathrm{~F}$ & L4 & 1.8 & G & M & 34.9 & 5.5 & 10.2 \\
\hline C3 & NISSAN & SENTRA & 1 & $\mathrm{~F}$ & L4 & 1.8 & G & A & 33.9 & 5.6 & 10.5 \\
\hline C3 & TOYOTA & $\mathrm{ECHO}$ & 1 & $\mathrm{~F}$ & L4 & 1.5 & G & M & 43.1 & 4.5 & 8.3 \\
\hline C3 & TOYOTA & COROLLA & 1 & $\mathrm{~F}$ & L4 & 1.8 & G & M & 39.6 & 4.9 & 9.0 \\
\hline C3 & TOYOTA & $\mathrm{ECHO}$ & I & $\mathrm{F}$ & L4 & 1.5 & G & $A$ & 39.4 & 4.9 & 9.1 \\
\hline
\end{tabular}




\begin{tabular}{|c|c|c|c|c|c|c|c|c|c|c|c|}
\hline $\begin{array}{l}\text { Type- } \\
\text { Size } \\
\text { Class }\end{array}$ & Manufacturer/Division & Name & $\begin{array}{l}\text { Dom I } \\
\text { Imp }\end{array}$ & DR & Cyl & Disp & Fuel & Trans & MPG & GHG & $\begin{array}{l}\text { Oil } \\
\text { Use }\end{array}$ \\
\hline$\overline{\mathrm{C} 3}$ & TOYOTA & COROLLA & $\mathrm{T}$ & $\bar{F}$ & L4 & 1.8 & $\mathrm{G}$ & $\bar{A}$ & 37.4 & 5.1 & 9.6 \\
\hline $\mathrm{C} 3$ & TOYOTA & COROLLA & I & $\mathrm{F}$ & L4 & 1.8 & G & A & 34.9 & 5.5 & 10.2 \\
\hline C3 & VWA & GOLF TDI & I & $\mathrm{F}$ & L4 & 1.9 & D & M & 52.7 & 4.0 & 6.8 \\
\hline C3 & VWA & JETTA & I & $\mathrm{F}$ & L4 & 1.9 & D & $M$ & 52.7 & 4.0 & 6.8 \\
\hline C3 & VWA & GOLF TDI & I & $\mathrm{F}$ & L4 & 1.9 & $\mathrm{D}$ & $A$ & 44.7 & 4.7 & 8.0 \\
\hline C3 & VWA & JETTA & I & $\mathrm{F}$ & L4 & 1.9 & D & A & 44.7 & 4.7 & 8.0 \\
\hline \multicolumn{12}{|c|}{ Midsize Car } \\
\hline$\overline{\mathrm{C} 4}$ & DAEWOO & LANOS- 3Dr & I & $\mathrm{F}$ & L4 & 1.5 & $\mathrm{G}$ & $M$ & 34.8 & 5.5 & 10.3 \\
\hline $\mathrm{C} 4$ & DAEWOO & LANOS-3Dr & I & $\mathrm{F}$ & Ł4 & 1.6 & G & $M$ & 34.6 & 5.5 & 10.3 \\
\hline C4 & DAEWOO & LANOS- 3Dr & I & $\mathrm{F}$ & L4 & 1.5 & G & A & 32.9 & 5.8 & 10.8 \\
\hline C4 & DAEWOO & LANOS-4Dr & I & $\mathrm{F}$ & L4 & 1.6 & G & $M$ & 31.8 & 6.0 & 11.2 \\
\hline C4 & DAEWOO & LANOS-3Dr & I & $\mathrm{F}$ & L4 & 1.6 & G & $A$ & 31.2 & 6.1 & 11.4 \\
\hline C4 & DAEWOO & NUBIRA & I & $\mathrm{F}$ & L4 & 2.0 & G & $M$ & 30.1 & 6.3 & 11.8 \\
\hline C4 & DAEWOO & LANOS-4Dr & I & $\mathrm{F}$ & L4 & 1.6 & G & A & 29.7 & 6.4 & 12.0 \\
\hline $\mathrm{C} 4$ & DAEWOO & NUBIRA & I & $\mathrm{F}$ & L4 & 2.0 & G & $A$ & 29.5 & 6.5 & 12.1 \\
\hline C4 & DAEWOO & NUBIRA & I & $\mathrm{F}$ & L4 & 2.0 & G & $M$ & 30.1 & 6.3 & 11.8 \\
\hline C4 & DAEWOO & NUBIRA & I & $\mathrm{F}$ & L4 & 2.0 & G & A & 29.5 & 6.5 & 12.1 \\
\hline C4 & DCC - CHRYSLER & CIRRUS & $\mathrm{D}$ & $\mathrm{F}$ & L4 & 2.0 & G & $M$ & 34.3 & 5.6 & 10.4 \\
\hline C4 & DCC - PLYMOUTH & BREEZE & $\mathrm{D}$ & $\mathrm{F}$ & L4 & 2.0 & G & $M$ & 35.4 & 5.4 & 10.1 \\
\hline $\mathrm{C} 4$ & FMC - MAZDA & 626 & I & $\mathrm{F}$ & L4 & 2.0 & G & $M$ & 33.5 & 5.7 & 10.6 \\
\hline $\mathrm{C} 4$ & GMC - SAAB & SAAB 9-3 & I & $\mathrm{F}$ & L4 & 2.0 & G & $M$ & 28.6 & 6.7 & 12.5 \\
\hline C4 & GMC - SATURN & LS & $\mathrm{D}$ & $\mathrm{F}$ & L4 & 2.2 & G & $M$ & 31.9 & 6.0 & 11.2 \\
\hline C4 & GMC - SATURN & LS & $\mathrm{D}$ & $\mathrm{F}$ & L4 & 2.2 & G & A & 30.5 & 6.3 & 11.7 \\
\hline C4 & HONDA & ACCORD Ix & I & $\mathrm{F}$ & L4 & 2.3 & G & $M$ & 32.1 & 6.0 & 11.1 \\
\hline C4 & HONDA & ACCORD & I & $\mathrm{F}$ & L4 & 2.3 & G & $M$ & 30.4 & 6.3 & 11.8 \\
\hline $\mathrm{C} 4$ & HONDA & ACCORD Ix & I & $\mathrm{F}$ & L4 & 2.3 & G & $A$ & 29.9 & 6.4 & 11.9 \\
\hline C4 & HONDA & ACCORD & I & $\mathrm{F}$ & L4 & 2.3 & G & A & 28.6 & 6.7 & 12.5 \\
\hline C4 & TOYOTA & CAMRY CNG & I & $\mathrm{F}$ & L4 & 2.2 & NG & A & 30.2 & 5.4 & 1.8 \\
\hline C4 & TOYOTA & CAMRY & I & $\mathrm{F}$ & L4 & 2.2 & G & M & 31.0 & 6.2 & 11.5 \\
\hline C4 & TOYOTA & CAMRY & I & $\mathrm{F}$ & L4 & 2.2 & G & $A$ & 30.1 & 6.4 & 11.9 \\
\hline C4 & VWA & PASSAT & I & $\mathrm{F}$ & L4 & 1.8 & G & M & 31.3 & 6.1 & 11.4 \\
\hline \multicolumn{12}{|c|}{ Large Car } \\
\hline $\mathrm{C5}$ & DAEWOO & LEGANZA & I & $\mathrm{F}$ & L4 & 2.2 & G & $M$ & 27.6 & 6.9 & 12.9 \\
\hline C5 & DAEWOO & LEGANZA & I & $\mathrm{F}$ & L4 & 2.2 & G & $M$ & 27.6 & 6.9 & 12.9 \\
\hline C5 & DCC - CHRYSLER & INTREPID & $\mathrm{D}$ & $\mathrm{F}$ & V6 & 2.7 & G & A & 27.3 & 7.0 & 13.1 \\
\hline C5 & DCC - CHRYSLER & CONCORDE & $\mathrm{D}$ & $\mathrm{F}$ & V6 & 2.7 & G & A & 27.3 & 7.0 & 13.1 \\
\hline C5 & DCC - DODGE & INTREPID & $\mathrm{D}$ & $\mathrm{F}$ & V6 & 2.7 & G & A & 27.3 & 7.0 & 13.1 \\
\hline C5 & DCC - DODGE & INTREPID & $\mathrm{D}$ & $\mathrm{F}$ & V6 & 2.7 & G & A & 27.3 & 7.0 & 13.1 \\
\hline C5 & GMC - CHEVY & IMPALA & $\mathrm{D}$ & $\mathrm{F}$ & V6 & 3.4 & G & A & 27.5 & 6.9 & 13.0 \\
\hline C5 & TOYOTA & AVALON & I & $\mathrm{F}$ & V6 & 3.0 & G & A & 28.1 & 6.8 & 12.7 \\
\hline \multicolumn{12}{|c|}{ Two-seater } \\
\hline$\overline{\mathrm{C} 6}$ & DCC - MER-BENZ & $\begin{array}{l}\text { SLK } 230 \\
\text { KOMPRESSOR }\end{array}$ & $\mathrm{I}$ & $\mathrm{R}$ & L4 & 2.3 & G & $M$ & 28.7 & 6.7 & 12.5 \\
\hline C6 & DCC - MER-BENZ & $\begin{array}{l}\text { SLK } 230 \\
\text { KOMPRESSOR }\end{array}$ & I & $\mathrm{R}$ & L4 & 2.3 & G & $A$ & 29.2 & 6.5 & 12.2 \\
\hline C6 & FMC - MAZDA & MX-5 MIATA & I & $\mathrm{R}$ & L4 & 1.8 & G & $M$ & 31.4 & 6.1 & 11.4 \\
\hline C6 & FMC - MAZDA & MX-5 MIATA & I & $\mathrm{R}$ & L4 & 1.8 & $\mathrm{G}$ & $M$ & 30.2 & 6.3 & 11.8 \\
\hline C6 & FMC - MAZDA & MX-5 MIATA & I & $\mathrm{R}$ & L4 & 1.8 & G & A & 28.9 & 6.6 & 12.3 \\
\hline C6 & HONDA & INSIGHT & I & $\mathrm{F}$ & L3 & 1.0 & $\mathrm{G} / \mathrm{EL}$ & $M$ & 76.3 & 2.6 & 4.7 \\
\hline C6 & TOYOTA & MR2 & I & $\mathrm{F}$ & L4 & 1.8 & G & $M$ & 31.8 & 6.0 & 11.2 \\
\hline
\end{tabular}




\begin{tabular}{|c|c|c|c|c|c|c|c|c|c|c|c|}
\hline $\begin{array}{l}\text { Type- } \\
\text { Size } \\
\text { Class }\end{array}$ & Manufacturer/Division & Name & $\begin{array}{l}\text { Dom I } \\
\text { Imp }\end{array}$ & DR & Cyl & Disp & Fuel & Trans & IPG & GHG & $\begin{array}{l}\text { Oil } \\
\text { Use }\end{array}$ \\
\hline \multicolumn{12}{|c|}{ Small Wagon } \\
\hline $\mathrm{C} 7$ & FMC - FORD & FOCUS WAGON & $\mathrm{D}$ & $\mathrm{F}$ & L4 & 2.0 & $\mathrm{G}$ & $M$ & 36.2 & 5.3 & 9.9 \\
\hline $\mathrm{C} 7$ & GMC - SATURN & SW & $\mathrm{D}$ & $\mathrm{F}$ & L4 & 1.9 & G & M & 36.1 & 5.3 & 9.9 \\
\hline $\mathrm{C7}$ & GMC - SATURN & SW & $\mathrm{D}$ & $\mathrm{F}$ & L4 & 1.9 & $\mathrm{G}$ & A & 35.4 & 5.4 & $4 \quad 10.1$ \\
\hline $\mathrm{C7}$ & GMC - SATURN & sW & $\mathrm{D}$ & $\mathrm{F}$ & L4 & 1.9 & G & A & 33.9 & 5.7 & 10.5 \\
\hline $\mathrm{C} 7$ & SUZUKI & ESTEEM WAGON & I & $\mathrm{F}$ & L4 & 1.8 & G & $M$ & 35.1 & 5.5 & $5 \quad 10.2$ \\
\hline $\mathrm{C} 7$ & SUZUKI & ESTEEM WAGON & I & $\mathrm{F}$ & L4 & 1.6 & $G$ & A & 33.7 & 5.7 & 10.6 \\
\hline \multicolumn{12}{|c|}{ Midsize Wagon } \\
\hline $\mathrm{C} 8$ & FMC - VOLVO & V70 & $\mathrm{I}$ & $\mathrm{F}$ & L5 & 2.4 & $\mathrm{G}$ & $M$ & 27.8 & 6.8 & 312.8 \\
\hline $\mathrm{C} 8$ & FMC - VOLVO & V70 & I & $\mathrm{F}$ & L5 & 2.4 & G & A & 27.3 & 7.0 & 13.1 \\
\hline $\mathrm{C} 8$ & GMC - SATURN & LW & $\mathrm{D}$ & $\mathrm{F}$ & L4 & 2.2 & $\mathrm{G}$ & A & 29.4 & 6.5 & $5 \quad 12.1$ \\
\hline $\mathrm{C} 8$ & VWA & PASSAT WAGON & I & $\mathrm{F}$ & L4 & 1.8 & G & $M$ & 31.3 & 6.1 & 11.4 \\
\hline $\mathrm{C} 8$ & VWA & PASSAT WAGON & I & $\mathrm{F}$ & L4 & 1.8 & $G$ & A & 27.4 & 7.0 & 13.1 \\
\hline \multicolumn{12}{|c|}{ Compact Pickup } \\
\hline$\overline{\mathrm{T} 1}$ & DCC - DODGE & DAKOTA 2WD & $\mathrm{D}$ & $\mathrm{R}$ & L4 & 2.5 & $G$ & $M$ & 25.7 & 7.4 & $4 \quad 13.9$ \\
\hline T1 & FMC - FORD & RANGER 4X2 EV & $\mathrm{D}$ & $\mathrm{R}$ & E0 & 2.5 & EL & A & 29.7 & 5.2 & 1.8 \\
\hline T1 & FMC - FORD & RANGER 4X2 & $\mathrm{D}$ & $\mathrm{R}$ & L4 & 2.5 & G & $M$ & 28.3 & 6.7 & $7 \quad 12.6$ \\
\hline T1 & FMC - FORD & RANGER 4X2 & $\mathrm{D}$ & $\mathrm{R}$ & L4 & 2.5 & G & A & 25.5 & 7.5 & $5 \quad 14.0$ \\
\hline T1 & GMC - CHEVROLET & S10 P/U 2WD & $\mathrm{D}$ & $\mathrm{R}$ & L4 & 2.2 & G & $M$ & 29.6 & 6.5 & $5 \quad 12.1$ \\
\hline T1 & GMC - CHEVROLET & S10 P/U 2WD & $\mathrm{D}$ & $\mathrm{R}$ & L4 & 2.2 & G & A & 25.2 & 7.5 & $5 \quad 14.2$ \\
\hline T1 & GMC & SONOMA 2WD & I & $\mathrm{R}$ & L4 & 2.2 & G & M & 29.6 & 6.5 & $5 \quad 12.1$ \\
\hline T1 & ISUZU & HOMBRE P/U 2WD & I & $\mathrm{R}$ & L4 & 2.2 & G & M & 28.4 & 6.7 & 712.6 \\
\hline T1 & NISSAN & $\begin{array}{l}\text { FRONTIER TRUCK } \\
2 \mathrm{WD}\end{array}$ & I & $\mathrm{R}$ & L4 & 2.4 & G & $M$ & 28.2 & 6.8 & $3 \quad 12.7$ \\
\hline $\mathrm{T} 1$ & NISSAN & $\begin{array}{l}\text { FRONTIER TRUCK } \\
2 \mathrm{WD}\end{array}$ & I & $\mathrm{R}$ & L4 & 2.4 & G & A & 25.0 & 7.6 & 14.3 \\
\hline T1 & TKM - MAZDA & MAZDA 4X2 & I & $\mathrm{R}$ & L4 & 2.5 & G & $\mathrm{M}$ & 28.3 & 6.7 & $7 \quad 12.6$ \\
\hline $\mathrm{T} 1$ & TKM - MAZDA & MAZDA 4X2 & I & $\mathrm{R}$ & L4 & 2.5 & $\mathrm{G}$ & A & 25.5 & 7.5 & $5 \quad 14.0$ \\
\hline T1 & TOYOTA & $\begin{array}{l}\text { TOYOTA TACOMA } \\
2 \mathrm{WD}\end{array}$ & I & $\mathrm{R}$ & L4 & 2.4 & $\mathrm{G}$ & $M$ & 27.4 & 7.0 & 13.1 \\
\hline $\mathrm{T} 1$ & TOYOTA & $\begin{array}{l}\text { TOYOTA TACOMA } \\
2 \text { WD }\end{array}$ & I & $\mathrm{R}$ & L4 & 2.4 & G & A & 26.2 & 7.2 & 13.6 \\
\hline \multicolumn{12}{|c|}{ Standard Pickup } \\
\hline $\mathrm{T} 2$ & FMC - FORD & $\mathrm{F} 1504 \mathrm{X} 2$ & $\mathrm{D}$ & $R$ & V6 & 4.2 & G & $\mathrm{A}$ & 20.7 & 9.1 & 17.3 \\
\hline T2 & FMC - FORD & $\mathrm{F} 1504 \mathrm{X} 2$ & $\mathrm{D}$ & $\mathrm{R}$ & V6 & 4.2 & G & M & 21.1 & 9.0 & 16.9 \\
\hline $\mathrm{T} 2$ & GMC - CHEVROLET & $\begin{array}{l}\text { C1500 SILVERADO } \\
\text { 2WD }\end{array}$ & $\mathrm{D}$ & $\mathrm{R}$ & V6 & 4.3 & G & M & 22.5 & 8.4 & $+\quad 15.9$ \\
\hline T2 & GMC - CHEVROLET & $\begin{array}{l}\text { C1500 SILVERADO } \\
\text { 2WD }\end{array}$ & $\mathrm{D}$ & $\mathrm{R}$ & V6 & 4.3 & G & A & 20.8 & 9.1 & 17.2 \\
\hline $\mathrm{T} 2$ & GMC - CHEVROLET & $\begin{array}{l}\text { C1500 SILVERADO } \\
\text { 2WD }\end{array}$ & $\mathrm{D}$ & $\mathrm{R}$ & V8 & 4.8 & G & M & 20.8 & 9.1 & 17.2 \\
\hline T2 & GMC - CHEVROLET & $\begin{array}{l}\text { C1500 SILVERADO } \\
\text { 2WD }\end{array}$ & $\mathrm{D}$ & $\mathrm{R}$ & V8 & 4.8 & $\mathrm{G}$ & A & 20.9 & 9.0 & 17.1 \\
\hline T2 & GMC & C1500 SIERRA 2WD & I & $\mathrm{R}$ & V6 & 4.3 & G & M & 22.8 & 8.3 & $3 \quad 15.7$ \\
\hline T2 & GMC & C1500 SIERRA 2WD & I & $\mathrm{R}$ & V6 & 4.3 & G & A & 20.8 & 9.1 & 17.2 \\
\hline T2 & GMC & C1500 SIERRA 2WD & I & $\mathrm{R}$ & V8 & 4.8 & G & A & 20.9 & 9.0 & 17.1 \\
\hline T2 & TOYOTA & TOYOTA TUNDRA 2WD & I & $\mathrm{R}$ & V6 & 3.4 & G & M & 20.8 & 9.1 & $1 \quad 17.2$ \\
\hline $\mathrm{T} 2$ & TOYOTA & TOYOTA TUNDRA 2WD & I & $\mathrm{R}$ & V6 & 3.4 & G & A & 20.6 & 9.2 & 217.4 \\
\hline \multicolumn{12}{|c|}{ Compact Van } \\
\hline$\overline{T 3}$ & DCC - DODGE & CARAVAN 2WD & $\mathrm{D}$ & $\mathrm{F}$ & L4 & 2.4 & $\mathrm{G}$ & A & 26.0 & 7.3 & 313.7 \\
\hline T3 & DCC - DODGE & CARAVAN 2WD & $\mathrm{D}$ & $\mathrm{F}$ & V6 & 3.3 & G & A & 24.3 & 7.8 & $3 \quad 14.7$ \\
\hline T3 & DCC - PLYMOUTH & $\begin{array}{l}\text { CARAVAN/VOYAGER } \\
\text { 2WD }\end{array}$ & D & $\mathrm{F}$ & L4 & 2.4 & G & A & 25.9 & 7.3 & 313.8 \\
\hline
\end{tabular}




\begin{tabular}{|c|c|c|c|c|c|c|c|c|c|c|c|}
\hline $\begin{array}{l}\text { Type- } \\
\text { Size } \\
\text { Class }\end{array}$ & Manufacturer/Division & Name & $\begin{array}{l}\text { Dom I } \\
\text { Imp }\end{array}$ & DR & Cyl & Disp $\quad F$ & Fuel & Trans & MPG & GHG & $\begin{array}{l}\text { Oil } \\
\text { Use }\end{array}$ \\
\hline T3 & GMC - CHEVROLET & VENTURE & $\mathrm{D}$ & $\mathrm{F}$ & V6 & 3.4 & G & A & 25.0 & 7.6 & 14.3 \\
\hline T3 & GMC - OLDSMOBILE & SILHOUETTE 2WD & $\mathrm{D}$ & $\mathrm{F}$ & V6 & 3.4 & G & $A$ & 25.0 & 7.6 & 14.3 \\
\hline T3 & GMC - PONTIAC & MONTANA FWD & $\mathrm{D}$ & $\mathrm{F}$ & V6 & 3.4 & G & A & 25.0 & 7.6 & 14.3 \\
\hline T3 & HONDA & ODYSSEY & I & $\mathrm{F}$ & V6 & 3.5 & G & A & 24.0 & 7.9 & 14.9 \\
\hline T3 & TOYOTA & SIENNA & I & $\mathrm{F}$ & V6 & 3.0 & G & A & 24.0 & 7.9 & 14.9 \\
\hline \multicolumn{12}{|c|}{ Standard Van } \\
\hline T4 & DCC - DODGE & B1500 VAN & $\mathrm{D}$ & $\mathrm{R}$ & V6 & 3.9 & G & $A$ & 18.5 & 10.2 & 19.3 \\
\hline T4 & FMC - FORD & E150 VAN & $\mathrm{D}$ & $\mathrm{R}$ & V8 & 4.6 & G & A & 19.5 & 9.7 & 18.4 \\
\hline T4 & FMC - FORD & E150 CLUB WAGON & $\mathrm{D}$ & $\mathrm{R}$ & V8 & 4.6 & G & A & 18.7 & 10.1 & 19.1 \\
\hline T4 & FMC - FORD & E150 VAN & $\mathrm{D}$ & $\mathrm{R}$ & V8 & 5.4 & G & A & 19.1 & 9.9 & 18.7 \\
\hline T4 & FMC - FORD & E150 VAN & $\mathrm{D}$ & $\mathrm{R}$ & V6 & 4.2 & G & A & 18.5 & 10.2 & 19.3 \\
\hline $\mathrm{T} 4$ & GMC - CHEVROLET & G15/25 CHEVY VAN & $\mathrm{D}$ & $\mathrm{R}$ & V6 & 4.3 & G & A & 18.8 & 10.1 & 19.0 \\
\hline T4 & GMC & G15/25SAVANA(C) & I & $\mathrm{R}$ & V6 & 4.3 & G & A & 18.9 & 10.0 & 18.9 \\
\hline \multicolumn{12}{|c|}{ Compact Utility } \\
\hline T5 & DCC - JEEP & CHEROKEE 2WD & 1 & $\mathrm{R}$ & L4 & 2.5 & G & $\mathrm{M}$ & 25.7 & 7.4 & 13.9 \\
\hline T5 & DCC - JEEP & CHEROKEE 2WD & I & $\mathrm{R}$ & L4 & 2.5 & G & A & 23.7 & 8.0 & 15.1 \\
\hline T5 & DCC - JEEP & CHEROKEE 2WD & I & $\mathrm{R}$ & L6 & 4.0 & G & M & 23.6 & 8.1 & 15.2 \\
\hline T5 & FMC - FORD & EXPLORER 4X2 & $\mathrm{D}$ & $\mathrm{R}$ & V6 & 4.0 & G & M & 23.0 & 8.2 & 15.5 \\
\hline T5 & GMC - CHEVROLET & BLAZER 2WD & $\mathrm{D}$ & $\mathrm{R}$ & V6 & 4.3 & G & M & 22.8 & 8.3 & 15.7 \\
\hline T5 & GMC & JIMMY 2WD & I & $\mathrm{R}$ & V6 & 4.3 & G & M & 22.8 & 8.3 & 15.7 \\
\hline T5 & HONDA & CR-V & I & $\mathrm{R}$ & L4 & 2.0 & G & A & 27.5 & 6.9 & 13.0 \\
\hline T5 & HONDA & CR-V & I & 4 & L4 & 2.0 & G & A & 27.1 & 7.0 & 13.2 \\
\hline T5 & HONDA & CR-V & I & 4 & L4 & 2.0 & G & $\mathrm{M}$ & 27.1 & 7.0 & 13.2 \\
\hline T5 & ISUZU & RODEO 2WD & I & $\mathrm{R}$ & L4 & 2.2 & G & $\mathrm{M}$ & 24.9 & 7.6 & 14.4 \\
\hline T5 & ISUZU & AMIGO 2WD & I & $\mathrm{R}$ & L4 & 2.2 & G & $\mathrm{M}$ & 24.8 & 7.6 & 14.4 \\
\hline T5 & MITSUBISHI & $\begin{array}{l}\text { MONTERO } \\
\text { SPORT/NATIVA }\end{array}$ & I & $\mathrm{R}$ & L4 & 2.3 & G & M & 26.5 & 7.2 & 13.5 \\
\hline T5 & MITSUBISHI & $\begin{array}{l}\text { MONTERO } \\
\text { SPORT/NATIVA }\end{array}$ & I & $\mathrm{R}$ & L4 & 2.3 & G & A & 24.2 & 7.8 & 14.7 \\
\hline T5 & NISSAN & XTERRA 2WD & I & $\mathrm{R}$ & L4 & 2.4 & G & $\mathrm{M}$ & 24.2 & 7.9 & 14.8 \\
\hline T5 & SUBARU & FORESTER & I & 4 & $\mathrm{HO} 4$ & 2.5 & G & $\mathrm{M}$ & 27.9 & 6.8 & 12.8 \\
\hline T5 & SUBARU & FORESTER & I & 4 & $\mathrm{HO} 4$ & 2.5 & G & A & 27.9 & 6.8 & 12.8 \\
\hline T5 & TOYOTA & 4RUNNER 2WD & I & $\mathrm{R}$ & L4 & 2.7 & G & A & 25.0 & 7.6 & 14.3 \\
\hline T5 & TOYOTA & 4RUNNER 2WD & I & $\mathrm{R}$ & L4 & 2.7 & G & M & 23.8 & 8.0 & 15.0 \\
\hline T5 & TOYOTA & 4RUNNER 4WD & I & 4 & L4 & 2.7 & G & A & 23.3 & 8.2 & 15.3 \\
\hline \multicolumn{12}{|c|}{ Standard Utility } \\
\hline$\overline{T 6}$ & FMC - FORD & EXPEDITION 4X2 & $\mathrm{D}$ & $\mathrm{R}$ & V8 & 4.6 & G & $A$ & 19.5 & 9.7 & 18.4 \\
\hline T6 & GMC - CHEVROLET & C1500 TAHOE 2WD & $\mathrm{D}$ & $\mathrm{R}$ & V8 & 4.8 & G & A & 19.5 & 9.7 & 18.3 \\
\hline T6 & GMC - CHEVROLET & C1500 TAHOE 2WD & $\mathrm{D}$ & $\mathrm{R}$ & V8 & 5.3 & G & A & 18.4 & 10.3 & 19.4 \\
\hline T6 & GMC & C1500 YUKON 2WD & I & $\mathrm{R}$ & V8 & 4.8 & G & A & 19.5 & 9.7 & 18.3 \\
\hline T6 & GMC & C1500 YUKON 2WD & I & $\mathrm{R}$ & V8 & 5.3 & G & $A$ & 18.4 & 10.3 & 19.4 \\
\hline T6 & GMC & C1500 YUKON 2WD & 1 & $\mathrm{R}$ & V8 & 5.3 & G & A & 18.4 & 10.3 & 19.4 \\
\hline \multicolumn{12}{|c|}{ Mini Utility } \\
\hline $\mathrm{T7}$ & GMC - CHEVROLET & TRACKER CONV 2WD & $\mathrm{D}$ & $\mathrm{R}$ & L4 & 1.6 & $\mathrm{G}$ & $M$ & 31.1 & 6.2 & 11.5 \\
\hline T7 & GMC - CHEVROLET & TRACKER CONV 4X4 & $\mathrm{D}$ & 4 & L4 & 1.6 & G & M & 30.7 & 6.2 & 11.6 \\
\hline T7 & GMC - CHEVROLET & TRACKER CONV 2WD & $\mathrm{D}$ & $\mathrm{R}$ & L4 & 2.0 & G & A & 29.1 & 6.6 & 12.3 \\
\hline $\mathrm{T7}$ & SUZUKI & VITARA CONV 2WD & I & $\mathrm{R}$ & L4 & 1.6 & G & M & 31.1 & 6.1 & 11.5 \\
\hline $\mathrm{T7}$ & SUZUKI & VITARA CONV 4WD & I & 4 & L4 & 1.6 & G & $\mathrm{M}$ & 30.7 & 6.2 & 11.6 \\
\hline $\mathrm{T7}$ & TOYOTA & RAV4 2WD & I & $\mathrm{F}$ & L4 & 2.0 & G & $\mathrm{M}$ & 30.9 & 6.2 & 11.6 \\
\hline $\mathrm{T7}$ & TOYOTA & RAV4 2WD & I & $\mathrm{F}$ & L4 & 2.0 & G & A & 30.5 & 6.3 & 11.7 \\
\hline
\end{tabular}




$$
\text { B-39 }
$$


Energy Star Method 10 Vehicle List

Top $20 \%$ of vehicles by GHG in each type-size

\begin{tabular}{|c|c|c|c|c|c|c|c|c|c|c|c|}
\hline $\begin{array}{l}\text { Type- } \\
\text { Size } \\
\text { Class } \\
\end{array}$ & Manufacturer/Division & Name & $\begin{array}{l}\text { Dom I } \\
\text { Imp }\end{array}$ & DR & Cyl & Disp $F$ & Fuel & Trans & MPG & GHG & $\begin{array}{l}\text { Oil } \\
\text { Use }\end{array}$ \\
\hline \multicolumn{12}{|c|}{ Minicompact Car } \\
\hline$\overline{\mathrm{C} 1}$ & DCC - MER-BENZ & CLK 320 (CABRIOLET) & 1 & $\mathrm{R}$ & V6 & 3.2 & $\mathrm{G}$ & $A$ & 26.5 & 7.2 & 13.5 \\
\hline $\mathrm{C} 1$ & PORSCHE - PORSCH & 911 CARRERA & 1 & $\mathrm{R}$ & $\mathrm{H} 6$ & 3.4 & G & M & 23.2 & 8.2 & 15.4 \\
\hline $\mathrm{C} 1$ & VWA - AUDI & AUDI TT COUPE & 1 & 4 & L4 & 1.8 & G & M & 29.4 & 6.5 & 12.1 \\
\hline \multicolumn{12}{|c|}{ Subcompact Car } \\
\hline $\mathrm{C} 2$ & GMC - CHEVY & METRO & I & $\mathrm{F}$ & L3 & 1.0 & G & $\mathrm{M}$ & 48.9 & 4.0 & 7.3 \\
\hline $\mathrm{C} 2$ & GMC - CHEVY & METRO & 1 & $\mathrm{~F}$ & L4 & 1.3 & G & M & 45.5 & 4.2 & 7.8 \\
\hline $\mathrm{C} 2$ & GMC - CHEVY & METRO & 1 & $\mathrm{~F}$ & L4 & 1.3 & $\mathrm{G}$ & $A$ & 37.6 & 5.1 & 9.5 \\
\hline $\mathrm{C} 2$ & GMC - SATURN & SC & D & $\mathrm{F}$ & L4 & 1.9 & G & M & 38.1 & 5.0 & 9.4 \\
\hline $\mathrm{C} 2$ & GMC - SATURN & SC & D & $\mathrm{F}$ & L4 & 1.9 & G & $A$ & 36.1 & 5.3 & 9.9 \\
\hline $\mathrm{C} 2$ & HONDA & CIVIC HX & 1 & $\mathrm{~F}$ & L4 & 1.6 & G & M & 44.7 & 4.3 & 8.0 \\
\hline $\mathrm{C} 2$ & HONDA & CIVIC HX & 1 & $\mathrm{~F}$ & L4 & 1.6 & $\mathrm{G}$ & $A$ & 41.9 & 4.6 & 8.5 \\
\hline $\mathrm{C} 2$ & HONDA & CIVIC dx 4Dr & 1 & $\mathrm{~F}$ & L4 & 1.6 & G & M & 37.2 & 5.2 & 9.6 \\
\hline $\mathrm{C} 2$ & HONDA & CIVIC ex & 1 & $\mathrm{~F}$ & L4 & 1.6 & $\mathrm{G}$ & M & 37.2 & 5.2 & 9.6 \\
\hline $\mathrm{C} 2$ & HONDA & CIVIC vp & I & $\mathrm{F}$ & L4 & 1.6 & $\mathrm{G}$ & $A$ & 35.5 & 5.4 & 10.1 \\
\hline $\mathrm{C} 2$ & HONDA & CIVIC dx 4Dr & I & $\mathrm{F}$ & L4 & 1.6 & G & $A$ & 35.6 & 5.4 & 10.0 \\
\hline $\mathrm{C} 2$ & HONDA & CIVIC ex & I & $\mathrm{F}$ & L4 & 1.6 & G & $A$ & 35.5 & 5.4 & 10.1 \\
\hline $\mathrm{C} 2$ & MITSUBISHI & MIRAGE 2D & I & $\mathrm{F}$ & L4 & 1.5 & G & M & 41.7 & 4.6 & 8.6 \\
\hline $\mathrm{C} 2$ & MITSUBISHI & MIRAGE & I & $\mathrm{F}$ & L4 & 1.8 & $\mathrm{G}$ & M & 36.3 & 5.3 & 9.8 \\
\hline $\mathrm{C} 2$ & MITSUBISHI & MIRAGE 2D & I & $\mathrm{F}$ & L4 & 1.5 & $\mathrm{G}$ & $A$ & 36.7 & 5.2 & 9.7 \\
\hline $\mathrm{C} 2$ & SUZUKI & SWIFT & 1 & $\mathrm{~F}$ & L4 & 1.3 & G & M & 45.5 & 4.2 & 7.8 \\
\hline $\mathrm{C} 2$ & SUZUKI & ESTEEM & I & $\mathrm{F}$ & L4 & 1.6 & $\mathrm{G}$ & M & 38.6 & 5.0 & 9.3 \\
\hline $\mathrm{C} 2$ & SUZUKI & SWIFT & I & $\mathrm{F}$ & L4 & 1.3 & G & $A$ & 37.6 & 5.1 & 9.5 \\
\hline $\mathrm{C} 2$ & SUZUKI & ESTEEM & I & $\mathrm{F}$ & L4 & 1.8 & $\mathrm{G}$ & M & 36.2 & 5.3 & 9.9 \\
\hline $\mathrm{C} 2$ & VWA & NEW BEETLE & I & $\mathrm{F}$ & L4 & 1.9 & $\mathrm{D}$ & M & 52.7 & 4.0 & 6.8 \\
\hline $\mathrm{C} 2$ & VWA & NEW BEETLE & I & $\mathrm{F}$ & L4 & 1.9 & $\mathrm{D}$ & $A$ & 44.7 & 4.7 & 8.0 \\
\hline \multicolumn{12}{|c|}{ Compact Car } \\
\hline $\mathrm{C} 3$ & DCC - CHRYSLER & NEON & $\mathrm{D}$ & $\mathrm{F}$ & L4 & 2.0 & $\mathrm{G}$ & $M$ & 36.1 & 5.3 & 9.9 \\
\hline C3 & DCC - DODGE & NEON & $\mathrm{D}$ & $\mathrm{F}$ & L4 & 2.0 & G & M & 36.1 & 5.3 & 9.9 \\
\hline C3 & DCC - PLYMOUTH & NEON & $\mathrm{D}$ & $\mathrm{F}$ & L4 & 2.0 & G & M & 36.1 & 5.3 & 9.9 \\
\hline $\mathrm{C} 3$ & FMC - FORD & FOCUS & $\mathrm{D}$ & $\mathrm{F}$ & L4 & 2.0 & G & M & 35.7 & 5.4 & 10.0 \\
\hline $\mathrm{C} 3$ & FMC - FORD & ESCORT & $\mathrm{D}$ & $\mathrm{F}$ & L4 & 2.0 & $\mathrm{G}$ & M & 35.7 & 5.4 & 10.0 \\
\hline $\mathrm{C} 3$ & FMC - MAZDA & PROTEGE & 1 & $\mathrm{~F}$ & L4 & 1.6 & $\mathrm{G}$ & M & 36.5 & 5.3 & 9.8 \\
\hline C3 & GMC - CHEVY & PRIZM & I & $\mathrm{F}$ & L4 & 1.8 & G & M & 39.6 & 4.9 & 9.0 \\
\hline C3 & GMC - CHEVY & PRIZM & 1 & $\mathrm{~F}$ & L4 & 1.8 & G & $A$ & 37.5 & 5.1 & 9.5 \\
\hline C3 & GMC - CHEVY & PRIZM & 1 & $\mathrm{~F}$ & L4 & 1.8 & G & $A$ & 34.9 & 5.5 & 10.2 \\
\hline C3 & GMC - SATURN & SL & $\mathrm{D}$ & $\mathrm{F}$ & L4 & 1.9 & G & M & 38.7 & 5.0 & 9.2 \\
\hline C3 & GMC - SATURN & SL & $\mathrm{D}$ & $\mathrm{F}$ & L4 & 1.9 & G & M & 36.1 & 5.3 & 9.9 \\
\hline $\mathrm{C} 3$ & GMC - SATURN & SL & $\mathrm{D}$ & $\mathrm{F}$ & L4 & 1.9 & $\mathrm{G}$ & $A$ & 34.4 & 5.6 & 10.4 \\
\hline C3 & GMC - SATURN & SL & $\mathrm{D}$ & $\mathrm{F}$ & L4 & 1.9 & G & $A$ & 36.0 & 5.3 & 9.9 \\
\hline C3 & HYUNDAI & ACCENT & 1 & $\mathrm{~F}$ & L4 & 1.5 & G & M & 36.6 & 5.3 & 9.8 \\
\hline C3 & HYUNDAI & ACCENT & 1 & $\mathrm{~F}$ & L4 & 1.5 & G & $A$ & 34.2 & 5.6 & 10.4 \\
\hline $\mathrm{C} 3$ & NISSAN & SENTRA & 1 & $\mathrm{~F}$ & L4 & 1.8 & $\mathrm{G}$ & M & 34.9 & 5.5 & 10.2 \\
\hline $\mathrm{C} 3$ & NISSAN & SENTRA & I & $\mathrm{F}$ & L4 & 1.8 & $\mathrm{G}$ & $A$ & 33.9 & 5.6 & 10.5 \\
\hline C3 & TOYOTA & $\mathrm{ECHO}$ & I & $\mathrm{F}$ & L4 & 1.5 & $\mathrm{G}$ & M & 43.1 & 4.5 & 8.3 \\
\hline C3 & TOYOTA & COROLLA & I & $\mathrm{F}$ & L4 & 1.8 & G & $M$ & 39.6 & 4.9 & 9.0 \\
\hline C3 & TOYOTA & $\mathrm{ECHO}$ & 1 & $\mathrm{~F}$ & L4 & 1.5 & G & $A$ & 39.4 & 4.9 & 9.1 \\
\hline
\end{tabular}




\begin{tabular}{|c|c|c|c|c|c|c|c|c|c|c|c|}
\hline $\begin{array}{l}\text { Type- } \\
\text { Size } \\
\text { Class }\end{array}$ & Manufacturer/Division & Name & $\begin{array}{l}\text { Dom I } \\
\text { Imp }\end{array}$ & DR & Cyl & Disp & Fuel & Trans & MPG & GHG & $\begin{array}{l}\text { Oil } \\
\text { Use }\end{array}$ \\
\hline$\overline{\mathrm{C} 3}$ & TOYOTA & COROLLA & $\mathrm{I}$ & $\bar{F}$ & L4 & 1.8 & $\mathrm{G}$ & $A$ & 37.4 & 5.1 & 9.6 \\
\hline C3 & TOYOTA & COROLLA & I & $\mathrm{F}$ & L4 & 1.8 & G & A & 34.9 & 5.5 & 10.2 \\
\hline C3 & VWA & GOLF TDI & 1 & $\mathrm{~F}$ & L4 & 1.9 & $\mathrm{D}$ & M & 52.7 & 4.0 & 6.8 \\
\hline C3 & VWA & JETTA & 1 & $\mathrm{~F}$ & L4 & 1.9 & $\mathrm{D}$ & M & 52.7 & 4.0 & 6.8 \\
\hline C3 & VWA & GOLF TDI & 1 & $\mathrm{~F}$ & L4 & 1.9 & $\mathrm{D}$ & A & 44.7 & 4.7 & 8.0 \\
\hline C3 & VWA & JETTA & 1 & $\mathrm{~F}$ & L4 & 1.9 & D & A & 44.7 & 4.7 & 8.0 \\
\hline \multicolumn{12}{|c|}{ Midsize Car } \\
\hline $\mathrm{C4}$ & DAEWOO & LANOS- 3Dr & $\mathrm{I}$ & $F$ & L4 & 1.5 & $\mathrm{G}$ & $M$ & 34.8 & 5.5 & 10.3 \\
\hline C4 & DAEWOO & LANOS-3Dr & I & $\mathrm{F}$ & L4 & 1.6 & G & $M$ & 34.6 & 5.5 & 10.3 \\
\hline C4 & DAEWOO & LANOS- 3Dr & I & $\mathrm{F}$ & L4 & 1.5 & G & A & 32.9 & 5.8 & 10.8 \\
\hline C4 & DAEWOO & LANOS-4Dr & I & $\mathrm{F}$ & L4 & 1.6 & G & M & 31.8 & 6.0 & 11.2 \\
\hline C4 & DAEWOO & LANOS-3Dr & 1 & $\mathrm{~F}$ & L4 & 1.6 & G & A & 31.2 & 6.1 & 11.4 \\
\hline C4 & DAEWOO & NUBIRA & I & $\mathrm{F}$ & L4 & 2.0 & G & M & 30.1 & 6.3 & 11.8 \\
\hline C4 & DAEWOO & LANOS-4Dr & 1 & $\mathrm{~F}$ & L4 & 1.6 & G & A & 29.7 & 6.4 & 12.0 \\
\hline C4 & DAEWOO & NUBIRA & I & $\mathrm{F}$ & L4 & 2.0 & $\mathrm{G}$ & $A$ & 29.5 & 6.5 & 12.1 \\
\hline C4 & DAEWOO & NUBIRA & I & $\mathrm{F}$ & L4 & 2.0 & G & M & 30.1 & 6.3 & 11.8 \\
\hline C4 & DAEWOO & NUBIRA & I & $\mathrm{F}$ & L4 & 2.0 & G & $A$ & 29.5 & 6.5 & 12.1 \\
\hline C4 & DCC - CHRYSLER & CIRRUS & $\mathrm{D}$ & $\mathrm{F}$ & L4 & 2.0 & G & M & 34.3 & 5.6 & 10.4 \\
\hline C4 & DCC - PLYMOUTH & BREEZE & $D$ & $\mathrm{~F}$ & L4 & 2.0 & G & M & 35.4 & 5.4 & 10.1 \\
\hline C4 & FMC - MAZDA & 626 & 1 & $\mathrm{~F}$ & L4 & 2.0 & G & M & 33.5 & 5.7 & 10.6 \\
\hline C4 & GMC - SAAB & SAAB 9-3 & 1 & $\mathrm{~F}$ & L4 & 2.0 & G & M & 28.6 & 6.7 & 12.5 \\
\hline C4 & GMC - SATURN & LS & $D$ & $\mathrm{~F}$ & L4 & 2.2 & G & M & 31.9 & 6.0 & 11.2 \\
\hline C4 & GMC - SATURN & LS & $\mathrm{D}$ & $\mathrm{F}$ & L4 & 2.2 & G & A & 30.5 & 6.3 & 11.7 \\
\hline C4 & HONDA & ACCORD Ix & I & $\mathrm{F}$ & L4 & 2.3 & G & M & 32.1 & 6.0 & 11.1 \\
\hline C4 & HONDA & ACCORD & 1 & $\mathrm{~F}$ & L4 & 2.3 & G & M & 30.4 & 6.3 & 11.8 \\
\hline C4 & HONDA & ACCORD Ix & 1 & $\mathrm{~F}$ & L4 & 2.3 & G & A & 29.9 & 6.4 & 11.9 \\
\hline C4 & HONDA & ACCORD & I & $\mathrm{F}$ & L4 & 2.3 & G & A & 28.6 & 6.7 & 12.5 \\
\hline C4 & TOYOTA & CAMRY CNG & 1 & $\mathrm{~F}$ & L4 & 2.2 & NG & A & 30.2 & 5.4 & 1.8 \\
\hline C4 & TOYOTA & CAMRY & I & $\mathrm{F}$ & L4 & 2.2 & G & M & 31.0 & 6.2 & 11.5 \\
\hline C4 & TOYOTA & CAMRY & I & $\mathrm{F}$ & L4 & 2.2 & G & $A$ & 30.1 & 6.4 & 11.9 \\
\hline C4 & VWA & PASSAT & 1 & $\mathrm{~F}$ & L4 & 1.8 & G & M & 31.3 & 6.1 & 11.4 \\
\hline \multicolumn{12}{|c|}{ Large Car } \\
\hline C5 & DAEWOO & LEGANZA & $\mathrm{I}$ & $\mathrm{F}$ & L4 & 2.2 & G & $M$ & 27.6 & 6.9 & 12.9 \\
\hline C5 & DAEWOO & LEGANZA & 1 & $\mathrm{~F}$ & L4 & 2.2 & G & M & 27.6 & 6.9 & 12.9 \\
\hline C5 & DCC - CHRYSLER & INTREPID & D & $\mathrm{F}$ & V6 & 2.7 & G & A & 27.3 & 7.0 & 13.1 \\
\hline C5 & DCC - CHRYSLER & CONCORDE & $D$ & $\mathrm{~F}$ & V6 & 2.7 & G & A & 27.3 & 7.0 & 13.1 \\
\hline C5 & DCC - DODGE & INTREPID & $D$ & $\mathrm{~F}$ & V6 & 2.7 & G & $A$ & 27.3 & 7.0 & 13.1 \\
\hline C5 & DCC - DODGE & INTREPID & $D$ & $\mathrm{~F}$ & V6 & 2.7 & G & $A$ & 27.3 & 7.0 & 13.1 \\
\hline C5 & FMC - FORD & TAURUS FFV & $D$ & $\mathrm{~F}$ & V6 & 3.0 & $\mathrm{FF}$ & $A$ & 24.6 & 5.9 & 7.8 \\
\hline C5 & GMC - CHEVY & IMPALA & $D$ & $\mathrm{~F}$ & V6 & 3.4 & G & $A$ & 27.5 & 6.9 & 13.0 \\
\hline C5 & TOYOTA & AVALON & 1 & $\mathrm{~F}$ & V6 & 3.0 & G & $A$ & 28.1 & 6.8 & 12.7 \\
\hline \multicolumn{12}{|c|}{ Two-Seater } \\
\hline $\mathrm{C} 6$ & DCC - MER-BENZ & $\begin{array}{l}\text { SLK } 230 \\
\text { KOMPRESSOR }\end{array}$ & 1 & $R$ & L4 & 2.3 & $\mathrm{G}$ & $\mathrm{M}$ & 28.7 & 6.7 & 12.5 \\
\hline C6 & DCC - MER-BENZ & $\begin{array}{l}\text { SLK } 230 \\
\text { KOMPRESSOR }\end{array}$ & 1 & $\mathrm{R}$ & L4 & 2.3 & G & A & 29.2 & 6.5 & 12.2 \\
\hline C6 & FMC - MAZDA & MX-5 MIATA & 1 & $\mathrm{R}$ & L4 & 1.8 & G & M & 31.4 & 6.1 & 11.4 \\
\hline C6 & FMC - MAZDA & MX-5 MIATA & I & $\mathrm{R}$ & L4 & 1.8 & G & M & 30.2 & 6.3 & 11.8 \\
\hline C6 & FMC - MAZDA & MX-5 MIATA & 1 & $\mathrm{R}$ & L4 & 1.8 & G & $A$ & 28.9 & 6.6 & 12.3 \\
\hline C6 & HONDA & INSIGHT & I & $F$ & L3 & 1.0 & G/EL & M & 76.3 & 2.6 & 4.7 \\
\hline
\end{tabular}




\begin{tabular}{|c|c|c|c|c|c|c|c|c|c|c|c|}
\hline $\begin{array}{l}\text { Type- } \\
\text { Size } \\
\text { Class }\end{array}$ & Manufacturer/Division & Name & $\begin{array}{l}\text { Dom I } \\
\text { Imp }\end{array}$ & DR & Cyl & Disp F & Fuel & Trans & MPG & GHG & $\begin{array}{l}\text { Oil } \\
\text { Use }\end{array}$ \\
\hline$\overline{\mathrm{C} 6}$ & TOYOTA & MR2 & $\mathrm{I}$ & $\bar{F}$ & $\overline{\mathrm{L} 4}$ & 1.8 & $\bar{G}$ & $M$ & 31.8 & 6.0 & $\overline{11.2}$ \\
\hline \multicolumn{12}{|c|}{ Small Wagon } \\
\hline$\overline{C 7}$ & FMC - FORD & FOCUS WAGON & $D$ & $\mathrm{~F}$ & L4 & 2.0 & G & $M$ & 36.2 & 5.3 & $\overline{9.9}$ \\
\hline $\mathrm{C7}$ & GMC - SATURN & SW & $\mathrm{D}$ & $\mathrm{F}$ & L4 & 1.9 & G & M & 36.1 & 5.3 & 9.9 \\
\hline $\mathrm{C7}$ & GMC - SATURN & SW & $\mathrm{D}$ & $\mathrm{F}$ & L4 & 1.9 & G & A & 35.4 & 5.4 & 10.1 \\
\hline $\mathrm{C7}$ & GMC - SATURN & SW & $\mathrm{D}$ & $\mathrm{F}$ & L4 & 1.9 & G & A & 33.9 & 5.7 & 10.5 \\
\hline $\mathrm{C7}$ & SUZUKI & ESTEEM WAGON & 1 & $\mathrm{~F}$ & L4 & 1.8 & G & M & 35.1 & 5.5 & 10.2 \\
\hline $\mathrm{C7}$ & SUZUKI & ESTEEM WAGON & I & $\mathrm{F}$ & L4 & 1.6 & G & A & 33.7 & 5.7 & 10.6 \\
\hline \multicolumn{12}{|c|}{ Midsize Wagon } \\
\hline $\mathrm{C} 8$ & FMC - FORD & TAURUS WAGON FFV & $\mathrm{D}$ & $\mathrm{F}$ & V6 & 3.0 & $\mathrm{FF}$ & $A$ & 23.3 & 6.2 & 8.3 \\
\hline C8 & FMC - VOLVO & V70 & 1 & $\mathrm{~F}$ & L5 & 2.4 & G & M & 27.8 & 6.8 & 12.8 \\
\hline $\mathrm{C} 8$ & GMC - SATURN & LW & $\mathrm{D}$ & $\mathrm{F}$ & L4 & 2.2 & G & $A$ & 29.4 & 6.5 & 12.1 \\
\hline $\mathrm{C} 8$ & VWA & PASSAT WAGON & 1 & $\mathrm{~F}$ & L4 & 1.8 & G & $M$ & 31.3 & 6.1 & 11.4 \\
\hline $\mathrm{C} 8$ & VWA & PASSAT WAGON & I & $\mathrm{F}$ & L4 & 1.8 & G & A & 27.4 & 7.0 & 13.1 \\
\hline \multicolumn{12}{|c|}{ Compact Pickup } \\
\hline $\mathrm{T} 1$ & FMC - FORD & RANGER 4X2 EV & $\mathrm{D}$ & $R$ & E0 & 2.5 & EL & A & 29.7 & 5.2 & 1.8 \\
\hline T1 & FMC - FORD & RANGER 4X2 & D & $\mathrm{R}$ & L4 & 2.5 & G & M & 28.3 & 6.7 & 12.6 \\
\hline T1 & GMC - CHEVROLET & S10 P/U 2WD & $\mathrm{D}$ & $\mathrm{R}$ & L4 & 2.2 & $\mathrm{FF}$ & $M$ & 23.9 & 5.9 & 7.6 \\
\hline T1 & GMC - CHEVROLET & S10 P/U 2WD & $\mathrm{D}$ & $\mathrm{R}$ & L4 & 2.2 & $\mathrm{FF}$ & $A$ & 21.2 & 6.7 & 8.7 \\
\hline T1 & GMC - CHEVROLET & S10 P/U 2WD & $\mathrm{D}$ & $\mathrm{R}$ & L4 & 2.2 & G & $M$ & 29.6 & 6.5 & 12.1 \\
\hline T1 & GMC & SONOMA 2WD & I & $\mathrm{R}$ & L4 & 2.2 & $\mathrm{FF}$ & M & 23.9 & 5.9 & 7.6 \\
\hline T1 & GMC & SONOMA 2WD & I & $\mathrm{R}$ & L4 & 2.2 & $\mathrm{FF}$ & $A$ & 21.2 & 6.7 & 8.7 \\
\hline T1 & GMC & SONOMA 2WD & 1 & $\mathrm{R}$ & L4 & 2.2 & G & M & 29.6 & 6.5 & 12.1 \\
\hline T1 & ISUZU & HOMBRE P/U 2WD & I & $\mathrm{R}$ & L4 & 2.2 & FF & M & 23.9 & 5.9 & 7.6 \\
\hline T1 & ISUZU & HOMBRE P/U 2WD & 1 & $\mathrm{R}$ & L4 & 2.2 & $\mathrm{FF}$ & $A$ & 21.2 & 6.7 & 8.7 \\
\hline T1 & ISUZU & HOMBRE P/U 2WD & I & $\mathrm{R}$ & L4 & 2.2 & G & $M$ & 28.4 & 6.7 & 12.6 \\
\hline T1 & NISSAN & $\begin{array}{l}\text { FRONTIER TRUCK } \\
2 \mathrm{WD}\end{array}$ & I & $\mathrm{R}$ & L4 & 2.4 & G & M & 28.2 & 6.8 & 12.7 \\
\hline T1 & TKM - MAZDA & MAZDA 4X2 & 1 & $\mathrm{R}$ & L4 & 2.5 & G & M & 28.3 & 6.7 & 12.6 \\
\hline T1 & TOYOTA & $\begin{array}{l}\text { TOYOTA TACOMA } \\
2 \mathrm{WD}\end{array}$ & 1 & $\mathrm{R}$ & L4 & 2.4 & G & M & 27.4 & 7.0 & 13.1 \\
\hline \multicolumn{12}{|c|}{ Standard Pickup } \\
\hline $\mathrm{T} 2$ & FMC - FORD & F150 4X2 & $\mathrm{D}$ & $R$ & V6 & 4.2 & G & A & 20.7 & 9.1 & 17.3 \\
\hline $\mathrm{T} 2$ & FMC - FORD & $\mathrm{F} 1504 \mathrm{X} 2$ & D & $\mathrm{R}$ & V6 & 4.2 & G & $M$ & 21.1 & 9.0 & 16.9 \\
\hline $\mathrm{T} 2$ & GMC - CHEVROLET & $\begin{array}{l}\text { C1500 SILVERADO } \\
\text { 2WD }\end{array}$ & $\mathrm{D}$ & $\mathrm{R}$ & V6 & 4.3 & G & M & 22.5 & 8.4 & 15.9 \\
\hline $\mathrm{T} 2$ & GMC - CHEVROLET & $\begin{array}{l}\text { C1500 SILVERADO } \\
\text { 2WD }\end{array}$ & D & $\mathrm{R}$ & V6 & 4.3 & G & A & 20.8 & 9.1 & 17.2 \\
\hline $\mathrm{T} 2$ & GMC - CHEVROLET & $\begin{array}{l}\text { C1500 SILVERADO } \\
\text { 2WD }\end{array}$ & $\mathrm{D}$ & $\mathrm{R}$ & V8 & 4.8 & G & M & 20.8 & 9.1 & 17.2 \\
\hline T2 & GMC - CHEVROLET & $\begin{array}{l}\text { C1500 SILVERADO } \\
\text { 2WD }\end{array}$ & $\mathrm{D}$ & $\mathrm{R}$ & V8 & 4.8 & G & $A$ & 20.9 & 9.0 & 17.1 \\
\hline T2 & GMC & C1500 SIERRA 2WD & I & $\mathrm{R}$ & V6 & 4.3 & G & M & 22.8 & 8.3 & 15.7 \\
\hline T2 & GMC & C1500 SIERRA 2WD & 1 & $\mathrm{R}$ & V6 & 4.3 & G & A & 20.8 & 9.1 & 17.2 \\
\hline $\mathrm{T} 2$ & GMC & C1500 SIERRA 2WD & 1 & $\mathrm{R}$ & V8 & 4.8 & G & A & 20.9 & 9.0 & 17.1 \\
\hline $\mathrm{T} 2$ & TOYOTA & $\begin{array}{l}\text { TOYOTA TUNDRA } \\
2 \mathrm{WD}\end{array}$ & I & $\mathrm{R}$ & V6 & 3.4 & G & M & 20.8 & 9.1 & 17.2 \\
\hline T2 & TOYOTA & $\begin{array}{l}\text { TOYOTA TUNDRA } \\
\text { 2WD }\end{array}$ & I & $\mathrm{R}$ & V6 & 3.4 & G & A & 20.6 & 9.2 & 17.4 \\
\hline \multicolumn{12}{|c|}{ Compact Van } \\
\hline T3 & DCC - CHRYSLER & $\begin{array}{l}\text { TOWN \& CNTRY 2WD } \\
\text { FFV }\end{array}$ & $\mathrm{D}$ & $\mathrm{F}$ & V6 & 3.3 & FF & A & 20.0 & 7.0 & 9.1 \\
\hline T3 & DCC - DODGE & CARAVAN 2WD FFV & $\mathrm{D}$ & $\mathrm{F}$ & V6 & 3.3 & $\mathrm{FF}$ & A & 19.9 & 7.1 & 9.2 \\
\hline T3 & DCC - DODGE & CARAVAN 2WD & D & $\mathrm{F}$ & L4 & 2.4 & G & A & 26.0 & 7.3 & 13.7 \\
\hline
\end{tabular}




\begin{tabular}{|c|c|c|c|c|c|c|c|c|c|c|c|}
\hline $\begin{array}{l}\text { Type- } \\
\text { Size } \\
\text { Class }\end{array}$ & Manufacturer/Division & Name & $\begin{array}{l}\text { Dom I } \\
\text { Imp }\end{array}$ & DR & Cyl & Disp & Fuel & Trans & MPG & GHG & $\begin{array}{l}\text { Oil } \\
\text { Use }\end{array}$ \\
\hline T3 & DCC - PLYMOUTH & $\begin{array}{l}\text { CARAVAN/VOYAGER } \\
\text { 2WD }\end{array}$ & $\bar{D}$ & $\bar{F}$ & L4 & 2.4 & G & $\bar{A}$ & 25.9 & 7.3 & 13.8 \\
\hline T3 & DCC - PLYMOUTH & $\begin{array}{l}\text { CARAVAN/VOYAGER } \\
\text { 2WD }\end{array}$ & $\mathrm{D}$ & $\mathrm{F}$ & V6 & 3.3 & FF & $A$ & 19.9 & 7.1 & 9.2 \\
\hline T3 & GMC - CHEVROLET & VENTURE & D & $\mathrm{F}$ & V6 & 3.4 & G & $A$ & 25.0 & 7.6 & 14.3 \\
\hline T3 & GMC - OLDSMOBILE & SILHOUETTE 2WD & $\mathrm{D}$ & $\mathrm{F}$ & V6 & 3.4 & $G$ & $A$ & 25.0 & 7.6 & 14.3 \\
\hline T3 & GMC - PONTIAC & MONTANA FWD & D & $\mathrm{F}$ & V6 & 3.4 & G & $A$ & 25.0 & 7.6 & 14.3 \\
\hline \multicolumn{12}{|c|}{ Standard Van } \\
\hline $\mathrm{T} 4$ & DCC - DODGE & B2500 VAN & $\mathrm{D}$ & $R$ & V8 & 5.2 & NG & $A$ & 15.5 & 10.0 & $\overline{3.5}$ \\
\hline T4 & FMC - FORD & E250 VAN NGV & D & $\mathrm{R}$ & V8 & 5.4 & NG & $A$ & 15.5 & 10.1 & 3.5 \\
\hline T4 & FMC - FORD & E150 VAN & D & $\mathrm{R}$ & V8 & 4.6 & G & $A$ & 19.5 & 9.7 & 18.4 \\
\hline $\mathrm{T} 4$ & FMC - FORD & E150 CLUB WAGON & D & $\mathrm{R}$ & V8 & 4.6 & G & $A$ & 18.7 & 10.1 & 19.1 \\
\hline $\mathrm{T} 4$ & FMC - FORD & E150 VAN & D & $\mathrm{R}$ & V8 & 5.4 & G & $A$ & 19.1 & 9.9 & 18.7 \\
\hline $\mathrm{T} 4$ & GMC - CHEVROLET & G15/25 CHEVY VAN & D & $\mathrm{R}$ & V6 & 4.3 & G & $A$ & 18.8 & 10.1 & 19.0 \\
\hline T4 & GMC & G15/25SAVANA(C) & I & $\mathrm{R}$ & V6 & 4.3 & G & $A$ & 18.9 & 10.0 & 18.9 \\
\hline \multicolumn{12}{|c|}{ Compact Utility } \\
\hline T5 & DCC - JEEP & CHEROKEE 2WD & I & $\mathrm{R}$ & L4 & 2.5 & G & $\mathrm{M}$ & 25.7 & 7.4 & 13.9 \\
\hline T5 & DCC - JEEP & CHEROKEE 2WD & I & $\mathrm{R}$ & L4 & 2.5 & G & $A$ & 23.7 & 8.0 & 15.1 \\
\hline T5 & DCC - JEEP & CHEROKEE 2WD & I & $\mathrm{R}$ & L6 & 4.0 & G & M & 23.6 & 8.1 & 15.2 \\
\hline T5 & FMC - FORD & EXPLORER 4X2 & D & $\mathrm{R}$ & V6 & 4.0 & G & M & 23.0 & 8.2 & 15.5 \\
\hline T5 & GMC - CHEVROLET & BLAZER 2WD & D & $\mathrm{R}$ & V6 & 4.3 & G & M & 22.8 & 8.3 & 15.7 \\
\hline T5 & GMC & JIMMY 2WD & 1 & $\mathrm{R}$ & V6 & 4.3 & G & M & 22.8 & 8.3 & 15.7 \\
\hline T5 & HONDA & CR-V & 1 & $\mathrm{R}$ & L4 & 2.0 & $G$ & $A$ & 27.5 & 6.9 & 13.0 \\
\hline T5 & HONDA & CR-V & 1 & 4 & L4 & 2.0 & G & $A$ & 27.1 & 7.0 & 13.2 \\
\hline T5 & HONDA & CR-V & I & 4 & L4 & 2.0 & $\mathrm{G}$ & M & 27.1 & 7.0 & 13.2 \\
\hline T5 & ISUZU & RODEO 2WD & 1 & $\mathrm{R}$ & L4 & 2.2 & G & M & 24.9 & 7.6 & 14.4 \\
\hline T5 & ISUZU & AMIGO 2WD & 1 & $\mathrm{R}$ & L4 & 2.2 & $\mathrm{G}$ & M & 24.8 & 7.6 & 14.4 \\
\hline T5 & MITSUBISHI & $\begin{array}{l}\text { MONTERO } \\
\text { SPORT/NATIVA }\end{array}$ & I & $\mathrm{R}$ & L4 & 2.3 & G & M & 26.5 & 7.2 & 13.5 \\
\hline T5 & MITSUBISHI & $\begin{array}{l}\text { MONTERO } \\
\text { SPORT/NATIVA }\end{array}$ & I & $\mathrm{R}$ & L4 & 2.3 & G & A & 24.2 & 7.8 & 14.7 \\
\hline T5 & NISSAN & XTERRA 2WD & 1 & $\mathrm{R}$ & L4 & 2.4 & G & M & 24.2 & 7.9 & 14.8 \\
\hline T5 & SUBARU & FORESTER & I & 4 & $\mathrm{HO} 4$ & 2.5 & $\mathrm{G}$ & M & 27.9 & 6.8 & 12.8 \\
\hline T5 & SUBARU & FORESTER & I & 4 & $\mathrm{HO} 4$ & 2.5 & $G$ & $A$ & 27.9 & 6.8 & 12.8 \\
\hline T5 & TOYOTA & 4RUNNER 2WD & 1 & $\mathrm{R}$ & L4 & 2.7 & $G$ & $A$ & 25.0 & 7.6 & 14.3 \\
\hline T5 & TOYOTA & 4RUNNER 2WD & 1 & $\mathrm{R}$ & L4 & 2.7 & $G$ & M & 23.8 & 8.0 & 15.0 \\
\hline T5 & TOYOTA & 4RUNNER 4WD & I & 4 & L4 & 2.7 & G & $A$ & 23.3 & 8.2 & 15.3 \\
\hline \multicolumn{12}{|c|}{ Standard Utility } \\
\hline T6 & FMC - FORD & EXPEDITION 4X2 & $\mathrm{D}$ & $\mathrm{R}$ & V8 & 4.6 & G & $A$ & 19.5 & 9.7 & 18.4 \\
\hline T6 & GMC - CHEVROLET & C1500 TAHOE 2WD & D & $\mathrm{R}$ & V8 & 4.8 & $G$ & $A$ & 19.5 & 9.7 & 18.3 \\
\hline T6 & GMC - CHEVROLET & C1500 TAHOE 2WD & $\mathrm{D}$ & $\mathrm{R}$ & V8 & 5.3 & G & $A$ & 18.4 & 10.3 & 19.4 \\
\hline T6 & GMC & C1500 YUKON 2WD & 1 & $\mathrm{R}$ & V8 & 4.8 & $G$ & $A$ & 19.5 & 9.7 & 18.3 \\
\hline T6 & GMC & C1500 YUKON 2WD & I & $\mathrm{R}$ & V8 & 5.3 & $G$ & $A$ & 18.4 & 10.3 & 19.4 \\
\hline T6 & GMC & C1500 YUKON 2WD & I & $\mathrm{R}$ & V8 & 5.3 & G & $A$ & 18.4 & 10.3 & 19.4 \\
\hline \multicolumn{12}{|c|}{ Mini Utility } \\
\hline $\mathrm{T7}$ & GMC - CHEVROLET & TRACKER CONV 2WD & $\mathrm{D}$ & $\mathrm{R}$ & L4 & 1.6 & G & M & 31.1 & 6.2 & 11.5 \\
\hline $\mathrm{T} 7$ & GMC - CHEVROLET & TRACKER CONV 4X4 & D & 4 & L4 & 1.6 & G & M & 30.7 & 6.2 & 11.6 \\
\hline $\mathrm{T} 7$ & GMC - CHEVROLET & TRACKER CONV 2WD & $\mathrm{D}$ & $\mathrm{R}$ & L4 & 2.0 & G & $A$ & 29.1 & 6.6 & 12.3 \\
\hline $\mathrm{T} 7$ & SUZUKI & VITARA CONV 2WD & 1 & $\mathrm{R}$ & L4 & 1.6 & G & M & 31.1 & 6.1 & 11.5 \\
\hline $\mathrm{T} 7$ & SUZUKI & VITARA CONV 4WD & 1 & 4 & L4 & 1.6 & G & M & 30.7 & 6.2 & 11.6 \\
\hline $\mathrm{T} 7$ & TOYOTA & RAV4 2WD & I & $\mathrm{F}$ & L4 & 2.0 & $G$ & M & 30.9 & 6.2 & 11.6 \\
\hline
\end{tabular}




\begin{tabular}{|c|c|c|c|c|c|c|c|c|c|c|c|}
\hline $\begin{array}{l}\text { Type- } \\
\text { Size } \\
\text { Class }\end{array}$ & Manufacturer/Division & Name & $\begin{array}{l}\text { Dom I } \\
\text { Imp }\end{array}$ & DR & Cyl & Disp & Fuel & Trans & MPG & GHG & $\begin{array}{l}\text { Oil } \\
\text { Use }\end{array}$ \\
\hline$\overline{\mathrm{T} 7}$ & TOYOTA & RAV4 2WD & $\mathrm{T}$ & $\mathrm{F}$ & L4 & 2.0 & $\bar{G}$ & $A$ & 30.5 & 6.3 & $\overline{11.7}$ \\
\hline
\end{tabular}


Energy Star Method 11 Vehicle List

Top $20 \%$ of vehicles by MPG in each market class

\begin{tabular}{|c|c|c|c|c|c|c|c|c|c|c|c|}
\hline $\begin{array}{l}\text { Type-Size } \\
\text { Class }\end{array}$ & Manufacturer/Division & Name & $\begin{array}{l}\text { Dom I } \\
\text { Imp }\end{array}$ & DR & Cyl & Disp & Fuel & Trans & MPG & GHG & $\begin{array}{l}\text { Oil } \\
\text { Use }\end{array}$ \\
\hline \multicolumn{12}{|l|}{ Small Cars } \\
\hline $\mathrm{C} 2$ & GMC - CHEVY & METRO & $\mathrm{I}$ & $\mathrm{F}$ & L3 & 1.0 & $\mathrm{G}$ & $\mathrm{M}$ & 48.9 & 4.0 & 7.3 \\
\hline $\mathrm{C} 2$ & GMC - CHEVY & METRO & I & $\mathrm{F}$ & L4 & 1.3 & $G$ & M & 45.5 & 4.2 & 7.8 \\
\hline C3 & GMC - CHEVY & PRIZM & I & $\mathrm{F}$ & L4 & 1.8 & G & M & 39.6 & 4.9 & 9.0 \\
\hline C3 & GMC - CHEVY & PRIZM & I & $\mathrm{F}$ & L4 & 1.8 & G & $A$ & 37.5 & 5.1 & 9.5 \\
\hline C2 & GMC - CHEVY & METRO & I & $\mathrm{F}$ & L4 & 1.3 & G & $A$ & 37.6 & 5.1 & 9.5 \\
\hline C3 & GMC - SATURN & SL & D & $\mathrm{F}$ & L4 & 1.9 & G & M & 38.7 & 5.0 & 9.2 \\
\hline $\mathrm{C} 2$ & GMC - SATURN & SC & $\mathrm{D}$ & $\mathrm{F}$ & L4 & 1.9 & G & M & 38.1 & 5.0 & 9.4 \\
\hline C6 & HONDA & INSIGHT & I & $\mathrm{F}$ & L3 & 1.0 & $\mathrm{G} / \mathrm{EL}$ & $M$ & 76.3 & 2.6 & 4.7 \\
\hline C2 & HONDA & CIVIC HX & I & $\mathrm{F}$ & L4 & 1.6 & $\mathrm{G}$ & M & 44.7 & 4.3 & 8.0 \\
\hline C2 & HONDA & CIVIC HX & 1 & $\mathrm{~F}$ & L4 & 1.6 & G & A & 41.9 & 4.6 & 8.5 \\
\hline $\mathrm{C} 2$ & MITSUBISHI & MIRAGE 2D & I & $\mathrm{F}$ & L4 & 1.5 & G & M & 41.7 & 4.6 & 8.6 \\
\hline C2 & SUZUKI & SWIFT & I & $\mathrm{F}$ & L4 & 1.3 & G & $M$ & 45.5 & 4.2 & 7.8 \\
\hline C2 & SUZUKI & ESTEEM & I & $\mathrm{F}$ & L4 & 1.6 & $\mathrm{G}$ & M & 38.6 & 5.0 & 9.3 \\
\hline $\mathrm{C} 2$ & SUZUKI & SWIFT & I & $\mathrm{F}$ & L4 & 1.3 & G & A & 37.6 & 5.1 & 9.5 \\
\hline C3 & TOYOTA & $\mathrm{ECHO}$ & I & $\mathrm{F}$ & L4 & 1.5 & $\mathrm{G}$ & M & 43.1 & 4.5 & 8.3 \\
\hline C3 & TOYOTA & COROLLA & I & $\mathrm{F}$ & L4 & 1.8 & $\mathrm{G}$ & M & 39.6 & 4.9 & 9.0 \\
\hline C3 & TOYOTA & $\mathrm{ECHO}$ & I & $\mathrm{F}$ & L4 & 1.5 & G & A & 39.4 & 4.9 & 9.1 \\
\hline C3 & TOYOTA & COROLLA & I & $\mathrm{F}$ & L4 & 1.8 & G & $A$ & 37.4 & 5.1 & 9.6 \\
\hline C2 & VWA & NEW BEETLE & I & $\mathrm{F}$ & L4 & 1.9 & $D$ & M & 52.7 & 4.0 & 6.8 \\
\hline C3 & VWA & GOLF TDI & I & $\mathrm{F}$ & L4 & 1.9 & $D$ & M & 52.7 & 4.0 & 6.8 \\
\hline C3 & VWA & JETTA & I & $\mathrm{F}$ & L4 & 1.9 & $D$ & M & 52.7 & 4.0 & 6.8 \\
\hline C2 & VWA & NEW BEETLE & I & $\mathrm{F}$ & L4 & 1.9 & $D$ & A & 44.7 & 4.7 & 8.0 \\
\hline C3 & VWA & GOLF TDI & I & $\mathrm{F}$ & L4 & 1.9 & $D$ & $A$ & 44.7 & 4.7 & 8.0 \\
\hline C3 & VWA & JETTA & I & $\mathrm{F}$ & L4 & 1.9 & $D$ & A & 44.7 & 4.7 & 8.0 \\
\hline \multicolumn{12}{|c|}{ Family Sedans } \\
\hline C4 & DCC - CHRYSLER & CIRRUS & $\mathrm{D}$ & $\mathrm{F}$ & L4 & 2.0 & $\mathrm{G}$ & $M$ & 34.3 & 5.6 & $\overline{10.4}$ \\
\hline $\mathrm{C} 4$ & DCC - PLYMOUTH & BREEZE & $\mathrm{D}$ & $\mathrm{F}$ & L4 & 2.0 & $\mathrm{G}$ & M & 35.4 & 5.4 & 10.1 \\
\hline C3 & FMC - FORD & CONTOUR & $\mathrm{D}$ & $\mathrm{F}$ & L4 & 2.0 & G & M & 32.6 & 5.9 & 11.0 \\
\hline C3 & FMC - FORD & CONTOUR & D & $\mathrm{F}$ & L4 & 2.0 & G & $A$ & 30.3 & 6.3 & 11.8 \\
\hline C3 & FMC - FORD & CONTOUR & $\mathrm{D}$ & $\mathrm{F}$ & L4 & 2.0 & $\mathrm{G}$ & $A$ & 30.3 & 6.3 & 11.8 \\
\hline C4 & FMC - MAZDA & 626 & I & $\mathrm{F}$ & L4 & 2.0 & G & M & 33.5 & 5.7 & 10.6 \\
\hline C3 & FMC - MERCURY & MYSTIQUE & $\mathrm{D}$ & $\mathrm{F}$ & L4 & 2.0 & G & M & 32.4 & 5.9 & 11.0 \\
\hline C4 & GMC - SATURN & LS & D & $\mathrm{F}$ & L4 & 2.2 & $\mathrm{G}$ & M & 31.9 & 6.0 & 11.2 \\
\hline C4 & GMC - SATURN & LS & $\mathrm{D}$ & $\mathrm{F}$ & L4 & 2.2 & $\mathrm{G}$ & $A$ & 30.5 & 6.3 & 11.7 \\
\hline $\mathrm{C} 4$ & HONDA & ACCORD Ix & 1 & $\mathrm{~F}$ & L4 & 2.3 & G & M & 32.1 & 6.0 & 11.1 \\
\hline C4 & HONDA & ACCORD & 1 & $\mathrm{~F}$ & L4 & 2.3 & $\mathrm{G}$ & M & 30.4 & 6.3 & 11.8 \\
\hline C3 & NISSAN - INFINITY & INFINITI G20 & I & $\mathrm{F}$ & L4 & 2.0 & G & $M$ & 31.0 & 6.2 & 11.5 \\
\hline C3 & NISSAN - INFINITY & INFINITI G20 & I & $\mathrm{F}$ & L4 & 2.0 & G & A & 30.2 & 6.3 & 11.8 \\
\hline C3 & NISSAN & ALTIMA & I & $\mathrm{F}$ & L4 & 2.4 & $\mathrm{G}$ & M & 30.9 & 6.2 & 11.6 \\
\hline C4 & TOYOTA & CAMRY CNG & 1 & $\mathrm{~F}$ & L4 & 2.2 & NG & A & 30.2 & 5.4 & 1.8 \\
\hline C4 & TOYOTA & CAMRY & I & $\mathrm{F}$ & L4 & 2.2 & $\mathrm{G}$ & M & 31.0 & 6.2 & 11.5 \\
\hline $\mathrm{C} 4$ & TOYOTA & CAMRY & 1 & $F$ & L4 & 2.2 & G & A & 30.1 & 6.4 & 11.9 \\
\hline C2 & TOYOTA & $\begin{array}{l}\text { CAMRY SOLARA } \\
\text { CONVERT }\end{array}$ & I & $\mathrm{F}$ & L4 & 2.2 & $\mathrm{G}$ & A & 30.1 & 6.4 & 11.9 \\
\hline $\mathrm{C} 4$ & VWA & PASSAT & I & $\mathrm{F}$ & L4 & 1.8 & G & M & 31.3 & 6.1 & 11.4 \\
\hline \multicolumn{12}{|c|}{ Large Sedans } \\
\hline C5 & DCC - CHRYSLER & INTREPID & $\mathrm{D}$ & $\mathrm{F}$ & V6 & 2.7 & $\mathrm{G}$ & $A$ & 27.3 & 7.0 & $\overline{13.1}$ \\
\hline C5 & DCC - CHRYSLER & CONCORDE & $\mathrm{D}$ & $\mathrm{F}$ & V6 & 2.7 & G & A & 27.3 & 7.0 & 13.1 \\
\hline C5 & DCC - DODGE & INTREPID & D & $\mathrm{F}$ & V6 & 2.7 & G & A & 27.3 & 7.0 & 13.1 \\
\hline C5 & DCC - DODGE & INTREPID & $\mathrm{D}$ & $\mathrm{F}$ & V6 & 2.7 & G & $A$ & 27.3 & 7.0 & 13.1 \\
\hline C5 & TOYOTA & AVALON & 1 & $\mathrm{~F}$ & V6 & 3.0 & G & A & 28.1 & 6.8 & 12.7 \\
\hline
\end{tabular}




\begin{tabular}{|c|c|c|c|c|c|c|c|c|c|c|c|}
\hline $\begin{array}{l}\text { Type-Size } \\
\text { Class }\end{array}$ & Manufacturer/Division & Name & $\begin{array}{l}\text { Dom I } \\
\text { Imp }\end{array}$ & DR & Cyl & Disp & Fuel & Trans & MPG & GHG & $\begin{array}{l}\text { Oil } \\
\text { Use }\end{array}$ \\
\hline \multicolumn{12}{|c|}{ Upscale Sedans } \\
\hline $\mathrm{C} 2$ & BMW & 328i,Ci & I & $\mathrm{R}$ & L6 & 2.8 & G & $\mathrm{M}$ & 27.6 & 6.9 & 12.9 \\
\hline C3 & BMW & 328 is & I & $\mathrm{R}$ & L6 & 2.8 & G & $M$ & 27.6 & 6.9 & 13.0 \\
\hline C3 & DCC - MER-BENZ & C 230 KOMPRESSOR & I & $\mathrm{R}$ & L4 & 2.3 & G & A & 28.3 & 6.7 & 12.6 \\
\hline C2 & DCC - MER-BENZ & CLK 320 & I & $\mathrm{R}$ & V6 & 3.2 & G & $A$ & 27.5 & 6.9 & 13.0 \\
\hline C4 & FMC - VOLVO & S70 & I & $\mathrm{F}$ & L5 & 2.4 & G & $M$ & 28.1 & 6.8 & 12.7 \\
\hline C2 & GMC - SAAB & SAAB 9-3 CVT & I & $\mathrm{F}$ & L4 & 2.0 & G & M & 28.6 & 6.7 & 12.5 \\
\hline C4 & GMC - SAAB & SAAB 9-3 & I & $\mathrm{F}$ & L4 & 2.0 & $G$ & $M$ & 28.6 & 6.7 & 12.5 \\
\hline C4 & GMC - SAAB & SAAB 9-3 VIGGEN & I & $\mathrm{F}$ & L4 & 2.3 & $\mathrm{G}$ & $M$ & 27.5 & 6.9 & 13.0 \\
\hline C4 & GMC - SAAB & SAAB 9-3 VIGGEN & I & $\mathrm{F}$ & L4 & 2.3 & G & $M$ & 27.5 & 6.9 & 13.0 \\
\hline C4 & GMC - SAAB & SAAB 9-5 & I & $\mathrm{F}$ & L4 & 2.3 & G & $M$ & 27.6 & 6.9 & 12.9 \\
\hline C3 & VWA - AUDI & AUDI A4 & I & $\mathrm{F}$ & L4 & 1.8 & G & $M$ & 31.9 & 6.0 & 11.2 \\
\hline C3 & VWA - AUDI & AUDI A4 QUATTRO & I & 4 & L4 & 1.8 & G & M & 28.6 & 6.7 & 12.5 \\
\hline C3 & VWA - AUDI & AUDI A4 & I & $\mathrm{F}$ & L4 & 1.8 & G & $A$ & 28.0 & 6.8 & 12.8 \\
\hline \multicolumn{12}{|c|}{ Luxury Sedans } \\
\hline C3 & BMW & $528 \mathrm{i}$ & I & $\mathrm{R}$ & L6 & 2.8 & G & $\mathrm{M}$ & 27.6 & 6.9 & 12.9 \\
\hline C3 & BMW & 528iA R1 & I & $\mathrm{R}$ & L6 & 2.8 & G & $A$ & 24.4 & 7.8 & 14.6 \\
\hline C3 & BMW & $528 \mathrm{iA}$ & I & $\mathrm{R}$ & L6 & 2.8 & G & A & 24.2 & 7.8 & 14.7 \\
\hline C4 & DCC - MER-BENZ & E 320 & I & $\mathrm{R}$ & V6 & 3.2 & G & A & 27.9 & 6.8 & 12.8 \\
\hline C4 & DCC - MER-BENZ & E 320 4MATIC & I & 4 & V6 & 3.2 & G & A & 26.9 & 7.1 & 13.3 \\
\hline C4 & FMC - JAGUAR & JAGUAR S-TYPE 6 & I & $\mathrm{R}$ & V6 & 3.0 & G & $A$ & 24.1 & 7.9 & 14.8 \\
\hline C4 & TOYOTA - LEXUS & GS 300 & I & $\mathrm{R}$ & L6 & 3.0 & G & A & 24.7 & 7.7 & 14.4 \\
\hline C4 & TOYOTA - LEXUS & LS 400 & I & $\mathrm{R}$ & V8 & 4.0 & G & A & 24.3 & 7.8 & 14.7 \\
\hline \multicolumn{12}{|l|}{ Coupes } \\
\hline$\overline{\mathrm{C} 2}$ & FMC - FORD & ESCORT ZX2 & $\mathrm{D}$ & $\mathrm{F}$ & L4 & 2.0 & $\mathrm{G}$ & $\mathrm{M}$ & 33.0 & 5.8 & 10.8 \\
\hline C2 & FMC - FORD & ESCORT ZX2 & $\mathrm{D}$ & $\mathrm{F}$ & L4 & 2.0 & G & $A$ & 33.1 & 5.8 & 10.8 \\
\hline C2 & GMC - CHEVY & CAVALIER & $\mathrm{D}$ & $\mathrm{F}$ & L4 & 2.2 & G & M & 32.1 & 6.0 & 11.1 \\
\hline C2 & GMC - PONTIAC & SUNFIRE & $\mathrm{D}$ & $\mathrm{F}$ & L4 & 2.2 & G & M & 32.1 & 6.0 & 11.1 \\
\hline C2 & GMC - SATURN & SC & $\mathrm{D}$ & $\mathrm{F}$ & L4 & 1.9 & G & M & 35.4 & 5.4 & 10.1 \\
\hline C2 & GMC - SATURN & SC & $\mathrm{D}$ & $\mathrm{F}$ & L4 & 1.9 & G & M & 38.1 & 5.0 & 9.4 \\
\hline C2 & GMC - SATURN & SC & $\mathrm{D}$ & $\mathrm{F}$ & L4 & 1.9 & G & A & 33.9 & 5.7 & 10.5 \\
\hline C2 & GMC - SATURN & SC & $\mathrm{D}$ & $\mathrm{F}$ & L4 & 1.9 & G & $A$ & 36.1 & 5.3 & 9.9 \\
\hline C6 & HONDA & INSIGHT & I & $\mathrm{F}$ & L3 & 1.0 & G/EL & M & 76.3 & 2.6 & 4.7 \\
\hline C2 & HONDA & CIVIC HX & I & $\mathrm{F}$ & L4 & 1.6 & G & M & 44.7 & 4.3 & 8.0 \\
\hline C2 & HONDA & CIVIC HX & I & $\mathrm{F}$ & L4 & 1.6 & G & A & 41.9 & 4.6 & 8.5 \\
\hline C2 & HONDA & CIVIC ex & I & $\mathrm{F}$ & L4 & 1.6 & G & M & 37.2 & 5.2 & 9.6 \\
\hline C2 & HONDA & CIVIC ex & I & $\mathrm{F}$ & L4 & 1.6 & G & A & 35.5 & 5.4 & 10.1 \\
\hline C2 & HONDA & CIVIC si & I & $\mathrm{F}$ & L4 & 1.6 & G & M & 32.8 & 5.8 & 10.9 \\
\hline $\mathrm{C} 2$ & MITSUBISHI & MIRAGE 2D & I & $\mathrm{F}$ & L4 & 1.5 & G & M & 41.7 & 4.6 & 8.6 \\
\hline C2 & MITSUBISHI & MIRAGE 2D & 1 & $\mathrm{~F}$ & L4 & 1.5 & G & A & 36.7 & 5.2 & 9.7 \\
\hline \multicolumn{12}{|c|}{ Convertibles } \\
\hline$\overline{\mathrm{C} 6}$ & FMC - MAZDA & MX-5 MIATA & $\mathrm{I}$ & $\mathrm{R}$ & L4 & 1.8 & $\mathrm{G}$ & $\mathrm{M}$ & 31.4 & 6.1 & $\overline{11.4}$ \\
\hline C6 & FMC - MAZDA & MX-5 MIATA & I & $\mathrm{R}$ & L4 & 1.8 & G & M & 30.2 & 6.3 & 11.8 \\
\hline C2 & GMC - PONTIAC & SUNFIRE CNVT & $\mathrm{D}$ & $\mathrm{F}$ & L4 & 2.2 & G & $A$ & 30.7 & 6.2 & 11.6 \\
\hline C2 & GMC - PONTIAC & SUNFIRE & $\mathrm{D}$ & $\mathrm{F}$ & L4 & 2.4 & G & M & 30.8 & 6.2 & 11.6 \\
\hline C2 & MITSUBISHI & ECLIPSE & I & $\mathrm{F}$ & L4 & 2.4 & G & A & 30.1 & 6.3 & 11.9 \\
\hline C6 & TOYOTA & MR2 & I & $\mathrm{F}$ & L4 & 1.8 & G & M & 31.8 & 6.0 & 11.2 \\
\hline $\mathrm{C} 2$ & TOYOTA & $\begin{array}{l}\text { CAMRY SOLARA } \\
\text { CONVERT }\end{array}$ & I & $\mathrm{F}$ & L4 & 2.2 & G & A & 30.1 & 6.4 & 11.9 \\
\hline C2 & VWA & CABRIO & I & $\mathrm{F}$ & L4 & 2.0 & G & M & 31.2 & 6.1 & 11.5 \\
\hline \multicolumn{12}{|c|}{ Sporty Cars } \\
\hline C6 & FMC - MAZDA & MX-5 MIATA & $I$ & $\mathrm{R}$ & L4 & 1.8 & G & $M$ & 31.4 & 6.1 & 11.4 \\
\hline C6 & FMC - MAZDA & MX-5 MIATA & I & $\mathrm{R}$ & L4 & 1.8 & G & M & 30.2 & 6.3 & 11.8 \\
\hline C3 & FMC - MERCURY & COUGAR & $\mathrm{D}$ & $\mathrm{F}$ & L4 & 2.0 & G & $M$ & 32.4 & 5.9 & 11.0 \\
\hline
\end{tabular}




\begin{tabular}{|c|c|c|c|c|c|c|c|c|c|c|c|}
\hline $\begin{array}{l}\text { Type-Size } \\
\text { Class }\end{array}$ & Manufacturer/Division & Name & $\begin{array}{l}\text { Dom I } \\
\text { Imp }\end{array}$ & DR & Cyl & Disp $F$ & Fuel & Trans & MPG & GHG & $\begin{array}{l}\text { Oil } \\
\text { Use }\end{array}$ \\
\hline $\mathrm{C} 2$ & GMC - SATURN & SC & $\mathrm{D}$ & $\mathrm{F}$ & L4 & 1.9 & $\mathrm{G}$ & $\bar{M}$ & 35.4 & 5.4 & $\overline{10.1}$ \\
\hline C2 & GMC - SATURN & SC & $\mathrm{D}$ & $F$ & L4 & 1.9 & $\mathrm{G}$ & M & 38.1 & 5.0 & 9.4 \\
\hline C2 & GMC - SATURN & SC & $\mathrm{D}$ & $\mathrm{F}$ & L4 & 1.9 & $\mathrm{G}$ & $A$ & 33.9 & 5.7 & 10.5 \\
\hline $\mathrm{C} 2$ & GMC - SATURN & SC & D & $F$ & L4 & 1.9 & G & A & 36.1 & 5.3 & 9.9 \\
\hline C2 & HYUNDAI & TIBURON & 1 & $\mathrm{~F}$ & L4 & 2.0 & $\mathrm{G}$ & M & 31.1 & 6.1 & 11.5 \\
\hline $\mathrm{C} 2$ & MITSUBISHI & ECLIPSE & I & $\mathrm{F}$ & L4 & 2.4 & G & M & 30.1 & 6.3 & 11.9 \\
\hline $\mathrm{C} 2$ & MITSUBISHI & ECLIPSE & I & $F$ & L4 & 2.4 & G & A & 30.1 & 6.3 & 11.9 \\
\hline C2 & TOYOTA & CELICA & I & $\mathrm{F}$ & L4 & 1.8 & $\mathrm{G}$ & M & 35.2 & 5.4 & 10.1 \\
\hline C2 & TOYOTA & CELICA & I & $\mathrm{F}$ & L4 & 1.8 & $\mathrm{G}$ & $A$ & 34.9 & 5.5 & 10.2 \\
\hline $\mathrm{C} 6$ & TOYOTA & MR2 & 1 & $F$ & L4 & 1.8 & G & M & 31.8 & 6.0 & 11.2 \\
\hline C2 & TOYOTA & CELICA & 1 & $\mathrm{~F}$ & L4 & 1.8 & G & M & 30.8 & 6.2 & 11.6 \\
\hline C2 & TOYOTA & CELICA & I & $\mathrm{F}$ & L4 & 1.8 & G & $A$ & 30.1 & 6.4 & 11.9 \\
\hline \multicolumn{12}{|c|}{ Station Wagons } \\
\hline $\mathrm{C} 7$ & FMC - FORD & FOCUS WAGON & $\mathrm{D}$ & $\mathrm{F}$ & L4 & 2.0 & G & $\mathrm{M}$ & 36.2 & 5.3 & 9.9 \\
\hline $\mathrm{C7}$ & FMC - FORD & FOCUS WAGON & $\mathrm{D}$ & $\mathrm{F}$ & L4 & 2.0 & $\mathrm{G}$ & $A$ & 32.6 & 5.9 & 11.0 \\
\hline $\mathrm{C7}$ & FMC - FORD & FOCUS WAGON & $\mathrm{D}$ & $F$ & L4 & 2.0 & G & A & 32.6 & 5.9 & 11.0 \\
\hline $\mathrm{C} 7$ & GMC - SATURN & SW & $\mathrm{D}$ & $\mathrm{F}$ & L4 & 1.9 & G & M & 36.1 & 5.3 & 9.9 \\
\hline $\mathrm{C} 7$ & GMC - SATURN & SW & $\mathrm{D}$ & $F$ & L4 & 1.9 & G & A & 35.4 & 5.4 & 10.1 \\
\hline $\mathrm{C} 7$ & GMC - SATURN & SW & $\mathrm{D}$ & $\mathrm{F}$ & L4 & 1.9 & G & A & 33.9 & 5.7 & 10.5 \\
\hline $\mathrm{C} 7$ & HYUNDAI & ELANTRA WAGON & 1 & $F$ & L4 & 2.0 & G & M & 31.3 & 6.1 & 11.4 \\
\hline $\mathrm{C} 7$ & SUZUKI & ESTEEM WAGON & I & $F$ & L4 & 1.8 & G & M & 35.1 & 5.5 & 10.2 \\
\hline $\mathrm{C} 7$ & SUZUKI & ESTEEM WAGON & I & $\mathrm{F}$ & L4 & 1.6 & G & A & 33.7 & 5.7 & 10.6 \\
\hline $\mathrm{C7}$ & SUZUKI & ESTEEM WAGON & I & $F$ & L4 & 1.8 & G & A & 33.7 & 5.7 & 10.6 \\
\hline C8 & VWA & PASSAT WAGON & I & $\mathrm{F}$ & L4 & 1.8 & $\mathrm{G}$ & M & 31.3 & 6.1 & 11.4 \\
\hline \multicolumn{12}{|l|}{ Minivans } \\
\hline T3 & DCC - CHRYSLER & $\begin{array}{l}\text { TOWN \& COUNTRY } \\
\text { 2WD }\end{array}$ & $\mathrm{D}$ & $F$ & V6 & 3.3 & G & $A$ & 23.2 & 8.2 & 15.4 \\
\hline Т3 & DCC - DODGE & CARAVAN 2WD & $\mathrm{D}$ & $\mathrm{F}$ & L4 & 2.4 & G & $A$ & 26.0 & 7.3 & 13.7 \\
\hline T3 & DCC - DODGE & CARAVAN 2WD & $\mathrm{D}$ & $F$ & V6 & 3.3 & G & A & 24.3 & 7.8 & 14.7 \\
\hline T3 & DCC - DODGE & CARAVAN 2WD & $\mathrm{D}$ & $\mathrm{F}$ & V6 & 3.0 & G & A & 24.0 & 7.9 & 14.9 \\
\hline T3 & DCC - DODGE & $\begin{array}{l}\text { GRAND CARAVAN } \\
2 \mathrm{WD}\end{array}$ & $\mathrm{D}$ & $\mathrm{F}$ & V6 & 3.8 & G & $A$ & 23.0 & 8.2 & 15.5 \\
\hline T3 & DCC - PLYMOUTH & $\begin{array}{l}\text { CARAVAN/VOYAGER } \\
2 W D\end{array}$ & $\mathrm{D}$ & $\mathrm{F}$ & V6 & 3.3 & G & A & 24.0 & 7.9 & 14.9 \\
\hline T3 & DCC - PLYMOUTH & $\begin{array}{l}\text { CARAVAN/VOYAGER } \\
\text { 2WD }\end{array}$ & $\mathrm{D}$ & $\mathrm{F}$ & L4 & 2.4 & G & A & 25.9 & 7.3 & 13.8 \\
\hline Т3 & DCC - PLYMOUTH & $\begin{array}{l}\text { CARAVAN/VOYAGER } \\
\text { 2WD }\end{array}$ & D & $\mathrm{F}$ & V6 & 3.0 & G & A & 24.0 & 7.9 & 14.9 \\
\hline T3 & GMC - CHEVROLET & VENTURE & D & $\mathrm{F}$ & V6 & 3.4 & G & $A$ & 25.0 & 7.6 & 14.3 \\
\hline T3 & GMC - OLDSMOBILE & SILHOUETTE 2WD & $\mathrm{D}$ & $\mathrm{F}$ & V6 & 3.4 & G & A & 25.0 & 7.6 & 14.3 \\
\hline T3 & GMC - PONTIAC & MONTANA FWD & $\mathrm{D}$ & $\mathrm{F}$ & V6 & 3.4 & G & A & 25.0 & 7.6 & 14.3 \\
\hline T3 & HONDA & ODYSSEY & 1 & $\mathrm{~F}$ & V6 & 3.5 & G & A & 24.0 & 7.9 & 14.9 \\
\hline Т3 & TKM - MAZDA & MAZDA MPV & 1 & $\mathrm{R}$ & V6 & 2.5 & G & A & 23.1 & 8.2 & 15.5 \\
\hline T3 & TOYOTA & SIENNA & I & $\mathrm{F}$ & V6 & 3.0 & G & A & 24.0 & 7.9 & 14.9 \\
\hline \multicolumn{12}{|c|}{ Pickup Trucks } \\
\hline T1 & DCC - DODGE & DAKOTA 2WD & $\mathrm{D}$ & $\mathrm{R}$ & L4 & 2.5 & $\mathrm{G}$ & $\mathrm{M}$ & 25.7 & 7.4 & 13.9 \\
\hline T1 & FMC - FORD & RANGER 4X2 EV & $\mathrm{D}$ & $\mathrm{R}$ & E0 & 2.5 & EL & A & 29.7 & 5.2 & 1.8 \\
\hline T1 & FMC - FORD & RANGER 4X2 & $\mathrm{D}$ & $\mathrm{R}$ & L4 & 2.5 & $\mathrm{G}$ & M & 28.3 & 6.7 & 12.6 \\
\hline T1 & FMC - FORD & RANGER 4X2 & $\mathrm{D}$ & $\mathrm{R}$ & L4 & 2.5 & $\mathrm{G}$ & A & 25.5 & 7.5 & 14.0 \\
\hline T1 & FMC - FORD & RANGER 4X2 & $\mathrm{D}$ & $\mathrm{R}$ & V6 & 4.0 & G & M & 22.9 & 8.3 & 15.6 \\
\hline T1 & GMC - CHEVROLET & S10 P/U 2WD & $\mathrm{D}$ & $\mathrm{R}$ & L4 & 2.2 & $\mathrm{FF}$ & M & 23.9 & 5.9 & 7.6 \\
\hline T1 & GMC - CHEVROLET & S10 P/U 2WD & $\mathrm{D}$ & $\mathrm{R}$ & L4 & 2.2 & G & M & 29.6 & 6.5 & 12.1 \\
\hline T1 & GMC - CHEVROLET & S10 P/U 2WD & $\mathrm{D}$ & $\mathrm{R}$ & L4 & 2.2 & $\mathrm{G}$ & $A$ & 25.2 & 7.5 & 14.2 \\
\hline T1 & GMC - CHEVROLET & S10 PICKUP 2WD & $\mathrm{D}$ & $\mathrm{R}$ & V6 & 4.3 & G & M & 22.9 & 8.3 & 15.6 \\
\hline T1 & GMC & SONOMA 2WD & 1 & $\mathrm{R}$ & L4 & 2.2 & FF & M & 23.9 & 5.9 & 7.6 \\
\hline T1 & GMC & SONOMA 2WD & 1 & $\mathrm{R}$ & L4 & 2.2 & $\mathrm{G}$ & M & 29.6 & 6.5 & 12.1 \\
\hline
\end{tabular}




\begin{tabular}{|c|c|c|c|c|c|c|c|c|c|c|c|}
\hline $\begin{array}{l}\text { Type-Size } \\
\text { Class }\end{array}$ & Manufacturer/Division & Name & $\begin{array}{l}\text { Dom I } \\
\text { Imp }\end{array}$ & DR & Cyl & Disp & Fuel & Trans & MPG & GHG & $\begin{array}{l}\text { Oil } \\
\text { Use }\end{array}$ \\
\hline$\overline{T 1}$ & GMC & SONOMA 2WD & $\mathrm{T}$ & $\bar{R}$ & L4 & 2.2 & $\bar{G}$ & $A$ & 24.6 & 7.7 & $\overline{14.5}$ \\
\hline T1 & GMC & SONOMA 2WD & I & $\mathrm{R}$ & V6 & 4.3 & G & M & 22.9 & 8.3 & 15.6 \\
\hline T1 & ISUZU & HOMBRE P/U 2WD & 1 & $\mathrm{R}$ & L4 & 2.2 & FF & M & 23.9 & 5.9 & 7.6 \\
\hline T1 & ISUZU & HOMBRE P/U 2WD & I & $\mathrm{R}$ & L4 & 2.2 & G & M & 28.4 & 6.7 & 12.6 \\
\hline T1 & ISUZU & HOMBRE P/U 2WD & 1 & $\mathrm{R}$ & L4 & 2.2 & G & $A$ & 24.6 & 7.7 & 14.5 \\
\hline T1 & NISSAN & $\begin{array}{l}\text { FRONTIER TRUCK } \\
2 \mathrm{WD}\end{array}$ & 1 & $\mathrm{R}$ & L4 & 2.4 & G & M & 28.2 & 6.8 & 12.7 \\
\hline T1 & NISSAN & $\begin{array}{l}\text { FRONTIER TRUCK } \\
2 \mathrm{WD}\end{array}$ & 1 & $\mathrm{R}$ & L4 & 2.4 & G & A & 25.0 & 7.6 & 14.3 \\
\hline T1 & TKM - MAZDA & MAZDA 4X2 & I & $\mathrm{R}$ & L4 & 2.5 & $\mathrm{G}$ & M & 28.3 & 6.7 & 12.6 \\
\hline T1 & TKM - MAZDA & MAZDA 4X2 & I & $\mathrm{R}$ & L4 & 2.5 & $G$ & $A$ & 25.5 & 7.5 & 14.0 \\
\hline T1 & TOYOTA & $\begin{array}{l}\text { TOYOTA TACOMA } \\
2 \mathrm{WD}\end{array}$ & 1 & $\mathrm{R}$ & L4 & 2.4 & G & M & 27.4 & 7.0 & 13.1 \\
\hline T1 & TOYOTA & $\begin{array}{l}\text { TOYOTA TACOMA } \\
2 \mathrm{WD}\end{array}$ & 1 & $\mathrm{R}$ & L4 & 2.4 & G & A & 26.2 & 7.2 & 13.6 \\
\hline T1 & TOYOTA & $\begin{array}{l}\text { TOYOTA TACOMA } \\
2 \mathrm{WD}\end{array}$ & 1 & $\mathrm{R}$ & V6 & 3.4 & G & M & 24.3 & 7.8 & 14.7 \\
\hline T1 & TOYOTA & $\begin{array}{l}\text { TOYOTA TACOMA } \\
2 \text { WD }\end{array}$ & 1 & $\mathrm{R}$ & L4 & 2.7 & G & $A$ & 23.5 & 8.1 & 15.2 \\
\hline T1 & TOYOTA & $\begin{array}{l}\text { TOYOTA TACOMA } \\
4 \mathrm{WD}\end{array}$ & 1 & 4 & L4 & 2.7 & G & A & 23.1 & 8.2 & 15.4 \\
\hline \multicolumn{12}{|l|}{ SUVs } \\
\hline T5 & DCC - JEEP & CHEROKEE 2WD & 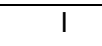 & $\mathrm{R}$ & L4 & 2.5 & G & $\mathrm{M}$ & 25.7 & 7.4 & 13.9 \\
\hline T7 & GMC - CHEVROLET & $\begin{array}{l}\text { TRACKER CONV } \\
\text { 2WD }\end{array}$ & D & $\mathrm{R}$ & L4 & 1.6 & G & M & 31.1 & 6.2 & 11.5 \\
\hline T7 & GMC - CHEVROLET & TRACKER CONV 4X4 & $\mathrm{D}$ & 4 & L4 & 1.6 & G & M & 30.7 & 6.2 & 11.6 \\
\hline T7 & GMC - CHEVROLET & $\begin{array}{l}\text { TRACKER CONV } \\
\text { 2WD }\end{array}$ & $\mathrm{D}$ & $\mathrm{R}$ & L4 & 2.0 & G & $A$ & 29.1 & 6.6 & 12.3 \\
\hline T7 & GMC - CHEVROLET & TRACKER VAN 2WD & D & $\mathrm{R}$ & L4 & 2.0 & G & A & 28.5 & 6.7 & 12.5 \\
\hline T7 & GMC - CHEVROLET & $\begin{array}{l}\text { TRACKER CONV } \\
\text { 2WD }\end{array}$ & D & $\mathrm{R}$ & L4 & 2.0 & G & M & 28.0 & 6.8 & 12.8 \\
\hline T7 & GMC - CHEVROLET & TRACKER VAN 2WD & D & $\mathrm{R}$ & L4 & 2.0 & G & M & 28.0 & 6.8 & 12.7 \\
\hline $\mathrm{T7}$ & GMC - CHEVROLET & TRACKER CONV 4X4 & $\mathrm{D}$ & 4 & L4 & 2.0 & G & $A$ & 27.9 & 6.8 & 12.8 \\
\hline $\mathrm{T} 7$ & GMC - CHEVROLET & TRACKER CONV 4X4 & $\mathrm{D}$ & 4 & L4 & 2.0 & G & M & 27.5 & 6.9 & 13.0 \\
\hline $\mathrm{T} 7$ & GMC - CHEVROLET & TRACKER VAN 4X4 & $\mathrm{D}$ & 4 & L4 & 2.0 & G & $A$ & 27.9 & 6.8 & 12.8 \\
\hline T7 & GMC - CHEVROLET & TRACKER VAN 4X4 & D & 4 & L4 & 2.0 & G & M & 27.4 & 7.0 & 13.0 \\
\hline T5 & HONDA & CR-V & 1 & $\mathrm{R}$ & L4 & 2.0 & G & A & 27.5 & 6.9 & 13.0 \\
\hline T5 & HONDA & CR-V & 1 & 4 & L4 & 2.0 & G & A & 27.1 & 7.0 & 13.2 \\
\hline T5 & HONDA & CR-V & 1 & 4 & L4 & 2.0 & G & M & 27.1 & 7.0 & 13.2 \\
\hline T5 & MITSUBISHI & $\begin{array}{l}\text { MONTERO } \\
\text { SPORT/NATIVA }\end{array}$ & 1 & $\mathrm{R}$ & L4 & 2.3 & G & M & 26.5 & 7.2 & 13.5 \\
\hline T5 & SUBARU & FORESTER & 1 & 4 & $\mathrm{HO} 4$ & 2.5 & G & M & 27.9 & 6.8 & 12.8 \\
\hline T5 & SUBARU & FORESTER & 1 & 4 & $\mathrm{HO} 4$ & 2.5 & G & A & 27.9 & 6.8 & 12.8 \\
\hline T7 & SUZUKI & VITARA CONV 2WD & 1 & $\mathrm{R}$ & L4 & 1.6 & G & M & 31.1 & 6.1 & 11.5 \\
\hline $\mathrm{T} 7$ & SUZUKI & VITARA CONV 4WD & 1 & 4 & L4 & 1.6 & G & M & 30.7 & 6.2 & 11.6 \\
\hline $\mathrm{T7}$ & SUZUKI & VITARA CONV 2WD & 1 & $\mathrm{R}$ & L4 & 2.0 & G & A & 27.8 & 6.8 & 12.8 \\
\hline $\mathrm{T} 7$ & SUZUKI & VITARA VAN 2WD & 1 & $\mathrm{R}$ & L4 & 2.0 & G & A & 27.7 & 6.9 & 12.9 \\
\hline $\mathrm{T7}$ & SUZUKI & VITARA CONV 2WD & 1 & $\mathrm{R}$ & L4 & 2.0 & G & M & 27.2 & 7.0 & 13.1 \\
\hline T7 & SUZUKI & VITARA VAN 2WD & 1 & $\mathrm{R}$ & L4 & 2.0 & G & M & 27.3 & 7.0 & 13.1 \\
\hline T7 & SUZUKI & VITARA CONV 4WD & 1 & 4 & L4 & 2.0 & G & A & 27.8 & 6.8 & 12.8 \\
\hline T7 & SUZUKI & VITARA CONV 4WD & 1 & 4 & L4 & 2.0 & G & M & 27.2 & 7.0 & 13.1 \\
\hline T7 & SUZUKI & VITARA VAN 4WD & 1 & 4 & L4 & 2.0 & G & A & 27.7 & 6.9 & 12.9 \\
\hline T7 & SUZUKI & VITARA VAN 4WD & I & 4 & L4 & 2.0 & G & M & 27.3 & 7.0 & 13.1 \\
\hline T7 & TOYOTA & RAV4 2WD & 1 & $\mathrm{~F}$ & L4 & 2.0 & G & M & 30.9 & 6.2 & 11.6 \\
\hline $\mathrm{T7}$ & TOYOTA & RAV4 2WD & 1 & $\mathrm{~F}$ & L4 & 2.0 & G & A & 30.5 & 6.3 & 11.7 \\
\hline $\mathrm{T} 7$ & TOYOTA & RAV4 4WD & 1 & 4 & L4 & 2.0 & G & A & 27.8 & 6.9 & 12.8 \\
\hline $\mathrm{T7}$ & TOYOTA & RAV4 4WD & I & 4 & L4 & 2.0 & G & M & 26.9 & 7.1 & 13.3 \\
\hline \multicolumn{12}{|c|}{ All-wheel drive \& 4-wheel drive } \\
\hline $\mathrm{C} 4$ & DCC - MER-BENZ & E 320 4MATIC & $\mathrm{I}$ & 4 & V6 & 3.2 & G & A & 26.9 & 7.1 & 13.3 \\
\hline
\end{tabular}




\begin{tabular}{|c|c|c|c|c|c|c|c|c|c|c|c|}
\hline $\begin{array}{l}\text { Type-Size } \\
\text { Class }\end{array}$ & Manufacturer/Division & Name & $\begin{array}{l}\text { Dom I } \\
\text { Imp }\end{array}$ & DR & Cyl & Disp $\quad F$ & Fuel & Trans & MPG & GHG & $\begin{array}{l}\text { Oil } \\
\text { Use }\end{array}$ \\
\hline $\mathrm{C} 8$ & DCC - MER-BENZ & $\begin{array}{l}\text { E } 320 \\
\text { 4MATIC(WAGON) }\end{array}$ & 1 & 4 & V6 & 3.2 & $G$ & $A$ & 25.9 & 7.3 & 13.8 \\
\hline $\mathrm{C} 2$ & FUJI - SUBARU & IMPREZA 2D & I & 4 & $\mathrm{H} 4$ & 2.2 & G & M & 29.3 & 6.5 & 12.2 \\
\hline $\mathrm{C} 7$ & FUJI - SUBARU & IMPREZA SW & I & 4 & $\mathrm{H} 4$ & 2.2 & G & A & 29.4 & 6.5 & 12.1 \\
\hline C2 & FUJI - SUBARU & IMPREZA 4D & 1 & 4 & $\mathrm{H} 4$ & 2.2 & G & A & 29.2 & 6.5 & 12.2 \\
\hline $\mathrm{C} 7$ & FUJI - SUBARU & IMPREZA SW & I & 4 & $\mathrm{H} 4$ & 2.2 & G & M & 29.3 & 6.5 & 12.2 \\
\hline $\mathrm{C} 2$ & FUJI - SUBARU & IMPREZA 2D & 1 & 4 & $\mathrm{H} 4$ & 2.5 & G & A & 29.2 & 6.5 & 12.2 \\
\hline C3 & FUJI - SUBARU & LEGACY 4D & 1 & 4 & $\mathrm{H} 4$ & 2.5 & G & $\mathrm{M}$ & 27.9 & 6.8 & 12.8 \\
\hline $\mathrm{C} 7$ & FUJI - SUBARU & LEGACY SW & I & 4 & $\mathrm{H} 4$ & 2.5 & G & M & 27.9 & 6.8 & 12.8 \\
\hline C3 & FUJI - SUBARU & LEGACY 4D & 1 & 4 & $\mathrm{H} 4$ & 2.5 & G & A & 27.9 & 6.8 & 12.8 \\
\hline $\mathrm{C} 7$ & FUJI - SUBARU & LEGACY SW & I & 4 & $\mathrm{H} 4$ & 2.5 & G & A & 27.8 & 6.8 & 12.8 \\
\hline C2 & FUJI - SUBARU & IMPREZA 4D & I & 4 & $\mathrm{H} 4$ & 2.5 & G & M & 27.4 & 6.9 & 13.0 \\
\hline C1 & VWA - AUDI & AUDI TT COUPE & 1 & 4 & L4 & 1.8 & G & M & 29.4 & 6.5 & 12.1 \\
\hline C3 & VWA - AUDI & AUDI A4 QUATTRO & 1 & 4 & L4 & 1.8 & $\mathrm{G}$ & $M$ & 28.6 & 6.7 & 12.5 \\
\hline $\mathrm{C} 7$ & VWA - AUDI & $\begin{array}{l}\text { AUDI A4 AVANT } \\
\text { QUATTRO }\end{array}$ & 1 & 4 & L4 & 1.8 & G & M & 28.6 & 6.7 & 12.5 \\
\hline C3 & VWA - AUDI & $\begin{array}{l}\text { AUDI A4 TT C } \\
\text { QUATTRO }\end{array}$ & 1 & 4 & L4 & 1.8 & G & $\mathrm{M}$ & 27.2 & 7.0 & 13.1 \\
\hline C3 & VWA - AUDI & AUDI A4 QUATTRO & 1 & 4 & L4 & 1.8 & G & A & 26.3 & 7.2 & 13.6 \\
\hline $\mathrm{C} 7$ & VWA - AUDI & $\begin{array}{l}\text { AUDI A4 AVANT } \\
\text { QUATTRO }\end{array}$ & 1 & 4 & L4 & 1.8 & G & A & 26.3 & 7.2 & 13.6 \\
\hline C3 & VWA - AUDI & AUDI A4 QUATTRO & 1 & 4 & V6 & 2.8 & G & M & 24.3 & 7.8 & 14.7 \\
\hline $\mathrm{C} 7$ & VWA - AUDI & $\begin{array}{l}\text { AUDI A4 AVANT } \\
\text { QUATTRO }\end{array}$ & 1 & 4 & V6 & 2.8 & G & M & 24.3 & 7.8 & 14.7 \\
\hline T7 & GMC - CHEVROLET & TRACKER CONV 4X4 & $\mathrm{D}$ & 4 & L4 & 1.6 & G & M & 30.7 & 6.2 & 11.6 \\
\hline T7 & GMC - CHEVROLET & TRACKER CONV 4X4 & $\mathrm{D}$ & 4 & L4 & 2.0 & G & A & 27.9 & 6.8 & 12.8 \\
\hline $\mathrm{T} 7$ & GMC - CHEVROLET & TRACKER CONV 4X4 & $\mathrm{D}$ & 4 & L4 & 2.0 & G & M & 27.5 & 6.9 & 13.0 \\
\hline $\mathrm{T7}$ & GMC - CHEVROLET & TRACKER VAN 4X4 & $\mathrm{D}$ & 4 & L4 & 2.0 & G & A & 27.9 & 6.8 & 12.8 \\
\hline T7 & GMC - CHEVROLET & TRACKER VAN 4X4 & $\mathrm{D}$ & 4 & L4 & 2.0 & G & M & 27.4 & 7.0 & 13.0 \\
\hline T5 & HONDA & CR-V & 1 & 4 & L4 & 2.0 & G & A & 27.1 & 7.0 & 13.2 \\
\hline T5 & HONDA & CR-V & 1 & 4 & L4 & 2.0 & G & M & 27.1 & 7.0 & 13.2 \\
\hline T7 & $\mathrm{KIA}$ & SPORTAGE & 1 & 4 & L4 & 2.0 & G & M & 24.6 & 7.7 & 14.5 \\
\hline T5 & SUBARU & FORESTER & I & 4 & $\mathrm{HO} 4$ & 2.5 & G & M & 27.9 & 6.8 & 12.8 \\
\hline T5 & SUBARU & FORESTER & 1 & 4 & $\mathrm{HO} 4$ & 2.5 & G & A & 27.9 & 6.8 & 12.8 \\
\hline $\mathrm{T7}$ & SUZUKI & VITARA CONV 4WD & 1 & 4 & L4 & 1.6 & G & M & 30.7 & 6.2 & 11.6 \\
\hline T7 & SUZUKI & VITARA CONV 4WD & 1 & 4 & L4 & 2.0 & G & A & 27.8 & 6.8 & 12.8 \\
\hline $\mathrm{T7}$ & SUZUKI & VITARA CONV 4WD & 1 & 4 & L4 & 2.0 & G & M & 27.2 & 7.0 & 13.1 \\
\hline $\mathrm{T} 7$ & SUZUKI & VITARA VAN 4WD & I & 4 & L4 & 2.0 & G & A & 27.7 & 6.9 & 12.9 \\
\hline $\mathrm{T7}$ & SUZUKI & VITARA VAN 4WD & I & 4 & L4 & 2.0 & G & M & 27.3 & 7.0 & 13.1 \\
\hline T7 & TOYOTA & RAV4 4WD & 1 & 4 & L4 & 2.0 & G & A & 27.8 & 6.9 & 12.8 \\
\hline $\mathrm{T} 7$ & TOYOTA & RAV4 4WD & 1 & 4 & L4 & 2.0 & G & $M$ & 26.9 & 7.1 & 13.3 \\
\hline
\end{tabular}


Energy Star Method 12 Vehicle List

Top $20 \%$ of vehicles by oil use per footprint

\begin{tabular}{|c|c|c|c|c|c|c|c|c|c|c|c|}
\hline $\begin{array}{l}\text { Type-Size } \\
\text { Class }\end{array}$ & Manufacturer/Division & Name & $\underset{\operatorname{Imp}}{\operatorname{Dom} I}$ & DR & Cyl & Disp & Fuel & Trans & MPG & GHG & $\begin{array}{l}\text { Oil } \\
\text { Use }\end{array}$ \\
\hline$\overline{\mathrm{C} 4}$ & DAEWOO & LANOS- 3Dr & $\mathrm{I}$ & $\bar{F}$ & $\mathrm{~L} 4$ & 1.5 & $\bar{G}$ & $\bar{M}$ & 34.8 & 5.5 & 10.3 \\
\hline C4 & DAEWOO & LANOS-3Dr & I & $\mathrm{F}$ & L4 & 1.6 & G & M & 34.6 & 5.5 & 10.3 \\
\hline C4 & DAEWOO & LANOS- 3Dr & 1 & $\mathrm{~F}$ & L4 & 1.5 & G & A & 32.9 & 5.8 & 10.8 \\
\hline C3 & DCC - CHRYSLER & NEON & D & $\mathrm{F}$ & L4 & 2.0 & G & M & 36.1 & 5.3 & 9.9 \\
\hline C4 & DCC - CHRYSLER & CIRRUS & D & $\mathrm{F}$ & L4 & 2.0 & G & M & 34.3 & 5.6 & 10.4 \\
\hline C3 & DCC - CHRYSLER & NEON & D & $\mathrm{F}$ & L4 & 2.0 & G & A & 31.9 & 6.0 & 11.2 \\
\hline C5 & DCC - CHRYSLER & INTREPID & D & $\mathrm{F}$ & V6 & 2.7 & G & A & 27.3 & 7.0 & 13.1 \\
\hline C5 & DCC - CHRYSLER & CONCORDE & D & $\mathrm{F}$ & V6 & 2.7 & G & A & 27.3 & 7.0 & 13.1 \\
\hline $\mathrm{C} 5$ & DCC - CHRYSLER & INTREPID & D & $\mathrm{F}$ & V6 & 3.2 & G & A & 25.5 & 7.5 & 14.0 \\
\hline C3 & DCC - DODGE & NEON & D & $\mathrm{F}$ & L4 & 2.0 & G & M & 36.1 & 5.3 & 9.9 \\
\hline C4 & DCC - DODGE & STRATUS & D & $\mathrm{F}$ & L4 & 2.0 & G & M & 28.3 & 6.7 & 12.6 \\
\hline C3 & DCC - DODGE & NEON & D & $\mathrm{F}$ & L4 & 2.0 & G & A & 31.9 & 6.0 & 11.2 \\
\hline C4 & DCC - DODGE & STRATUS & D & $\mathrm{F}$ & L4 & 2.0 & G & M & 28.3 & 6.7 & 12.6 \\
\hline $\mathrm{C} 5$ & DCC - DODGE & INTREPID & D & $\mathrm{F}$ & V6 & 2.7 & G & A & 27.3 & 7.0 & 13.1 \\
\hline $\mathrm{C} 5$ & DCC - DODGE & INTREPID & D & $\mathrm{F}$ & V6 & 2.7 & G & A & 27.3 & 7.0 & 13.1 \\
\hline $\mathrm{C} 5$ & DCC - DODGE & INTREPID & D & $\mathrm{F}$ & V6 & 3.2 & G & A & 25.5 & 7.5 & 14.0 \\
\hline C4 & DCC - MER-BENZ & E 320 & 1 & $\mathrm{R}$ & V6 & 3.2 & G & A & 27.9 & 6.8 & 12.8 \\
\hline C3 & DCC - PLYMOUTH & NEON & D & $\mathrm{F}$ & L4 & 2.0 & G & M & 36.1 & 5.3 & 9.9 \\
\hline C4 & DCC - PLYMOUTH & BREEZE & D & $\mathrm{F}$ & L4 & 2.0 & G & M & 35.4 & 5.4 & 10.1 \\
\hline C3 & DCC - PLYMOUTH & NEON & D & $\mathrm{F}$ & L4 & 2.0 & G & A & 31.9 & 6.0 & 11.2 \\
\hline C4 & DCC - PLYMOUTH & BREEZE & D & $\mathrm{F}$ & L4 & 2.0 & G & A & 28.3 & 6.7 & 12.6 \\
\hline $\mathrm{C} 5$ & FMC - FORD & CROWN VICTORIA NG & D & $\mathrm{R}$ & V8 & 4.6 & NG & A & 20.7 & 7.6 & 2.6 \\
\hline $\mathrm{C} 5$ & FMC - FORD & TAURUS FFV & D & $\mathrm{F}$ & V6 & 3.0 & $\mathrm{FF}$ & A & 24.6 & 5.9 & 7.8 \\
\hline $\mathrm{C} 8$ & FMC - FORD & TAURUS WAGON FFV & D & $\mathrm{F}$ & V6 & 3.0 & $\mathrm{FF}$ & A & 23.3 & 6.2 & 8.3 \\
\hline $\mathrm{C} 7$ & FMC - FORD & FOCUS WAGON & D & $\mathrm{F}$ & L4 & 2.0 & G & M & 36.2 & 5.3 & 9.9 \\
\hline C3 & FMC - FORD & FOCUS & D & $\mathrm{F}$ & L4 & 2.0 & G & M & 35.7 & 5.4 & 10.0 \\
\hline C3 & FMC - FORD & ESCORT & D & $\mathrm{F}$ & L4 & 2.0 & G & M & 35.7 & 5.4 & 10.0 \\
\hline C3 & FMC - FORD & FOCUS & D & $\mathrm{F}$ & L4 & 2.0 & G & A & 33.9 & 5.7 & 10.5 \\
\hline $\mathrm{C} 7$ & FMC - FORD & FOCUS WAGON & D & $\mathrm{F}$ & L4 & 2.0 & G & A & 32.6 & 5.9 & 11.0 \\
\hline C3 & FMC - FORD & ESCORT & D & $\mathrm{F}$ & L4 & 2.0 & G & A & 33.7 & 5.7 & 10.6 \\
\hline $\mathrm{C} 2$ & FMC - FORD & ESCORT ZX2 & D & $\mathrm{F}$ & L4 & 2.0 & G & M & 33.0 & 5.8 & 10.8 \\
\hline C3 & FMC - FORD & FOCUS 3D & D & $\mathrm{F}$ & L4 & 2.0 & G & M & 33.0 & 5.8 & 10.8 \\
\hline C3 & FMC - FORD & FOCUS 3D & D & $\mathrm{F}$ & L4 & 2.0 & G & A & 32.4 & 5.9 & 11.0 \\
\hline C2 & FMC - FORD & ESCORT ZX2 & D & $\mathrm{F}$ & L4 & 2.0 & G & A & 33.1 & 5.8 & 10.8 \\
\hline $\mathrm{C} 7$ & FMC - FORD & FOCUS WAGON & D & $\mathrm{F}$ & L4 & 2.0 & G & A & 32.6 & 5.9 & 11.0 \\
\hline C3 & FMC - FORD & CONTOUR & D & $\mathrm{F}$ & L4 & 2.0 & G & M & 32.6 & 5.9 & 11.0 \\
\hline C3 & FMC - FORD & CONTOUR & D & $\mathrm{F}$ & L4 & 2.0 & G & A & 30.3 & 6.3 & 11.8 \\
\hline C3 & FMC - FORD & CONTOUR & D & $\mathrm{F}$ & L4 & 2.0 & G & A & 30.3 & 6.3 & 11.8 \\
\hline C3 & FMC - MAZDA & PROTEGE & 1 & $\mathrm{~F}$ & L4 & 1.6 & G & A & 33.8 & 5.7 & 10.6 \\
\hline C4 & FMC - MAZDA & 626 & 1 & $\mathrm{~F}$ & L4 & 2.0 & G & M & 33.5 & 5.7 & 10.6 \\
\hline C3 & FMC - MAZDA & PROTEGE & I & $\mathrm{F}$ & L4 & 1.8 & G & M & 32.4 & 5.9 & 11.0 \\
\hline C3 & FMC - MAZDA & PROTEGE & I & $\mathrm{F}$ & L4 & 1.6 & G & M & 36.5 & 5.3 & 9.8 \\
\hline C3 & FMC - MERCURY & MYSTIQUE & D & $\mathrm{F}$ & L4 & 2.0 & G & M & 32.4 & 5.9 & 11.0 \\
\hline C3 & FMC - MERCURY & COUGAR & D & $\mathrm{F}$ & L4 & 2.0 & G & M & 32.4 & 5.9 & 11.0 \\
\hline C3 & FMC - MERCURY & MYSTIQUE & D & $\mathrm{F}$ & L4 & 2.0 & G & A & 29.6 & 6.5 & 12.1 \\
\hline C5 & GMC - BUICK & LESABRE & D & $\mathrm{F}$ & V6 & 3.8 & G & A & 26.6 & 7.2 & 13.5 \\
\hline C5 & GMC - BUICK & PARK AVENUE & D & $\mathrm{F}$ & V6 & 3.8 & G & A & 26.6 & 7.2 & 13.5 \\
\hline
\end{tabular}




\begin{tabular}{|c|c|c|c|c|c|c|c|c|c|c|c|}
\hline $\begin{array}{l}\text { Type-Size } \\
\text { Class }\end{array}$ & Manufacturer/Division & Name & $\begin{array}{c}\text { Dom I } \\
\text { Imp }\end{array}$ & DR & Cyl & Disp & Fuel & Trans & MPG & GHG & $\begin{array}{l}\text { Oil } \\
\text { Use }\end{array}$ \\
\hline $\mathrm{C} 2$ & GMC - CHEVY & METRO & $\mathrm{I}$ & $\mathrm{F}$ & L3 & 1.0 & $\mathrm{G}$ & $\mathrm{M}$ & 48.9 & 4.0 & 7.3 \\
\hline $\mathrm{C} 2$ & GMC - CHEVY & METRO & 1 & $\mathrm{~F}$ & L4 & 1.3 & G & M & 45.5 & 4.2 & 7.8 \\
\hline C3 & GMC - CHEVY & PRIZM & 1 & $\mathrm{~F}$ & L4 & 1.8 & G & M & 39.6 & 4.9 & 9.0 \\
\hline C3 & GMC - CHEVY & PRIZM & I & $\mathrm{F}$ & L4 & 1.8 & G & A & 37.5 & 5.1 & 9.5 \\
\hline $\mathrm{C} 2$ & GMC - CHEVY & METRO & I & $\mathrm{F}$ & L4 & 1.3 & G & A & 37.6 & 5.1 & 9.5 \\
\hline C3 & GMC - CHEVY & PRIZM & I & $\mathrm{F}$ & L4 & 1.8 & G & $A$ & 34.9 & 5.5 & 10.2 \\
\hline $\mathrm{C} 2$ & GMC - CHEVY & CAVALIER & $\mathrm{D}$ & $\mathrm{F}$ & L4 & 2.2 & G & $\mathrm{M}$ & 32.1 & 6.0 & 11.1 \\
\hline C2 & GMC - CHEVY & CAVALIER Z24 & $\mathrm{D}$ & $\mathrm{F}$ & L4 & 2.4 & G & M & 30.8 & 6.2 & 11.6 \\
\hline C5 & GMC - CHEVY & IMPALA & $\mathrm{D}$ & $\mathrm{F}$ & V6 & 3.4 & G & A & 27.5 & 6.9 & 13.0 \\
\hline C5 & GMC - CHEVY & IMPALA & $\mathrm{D}$ & $\mathrm{F}$ & V6 & 3.8 & G & A & 26.9 & 7.1 & 13.3 \\
\hline C3 & GMC - OLDSMOBILE & ALERO & $\mathrm{D}$ & $\mathrm{F}$ & L4 & 2.4 & G & M & 29.5 & 6.5 & 12.1 \\
\hline C3 & GMC - OLDSMOBILE & ALERO & $\mathrm{D}$ & $\mathrm{F}$ & L4 & 2.4 & G & A & 29.4 & 6.5 & 12.1 \\
\hline $\mathrm{C} 2$ & GMC - PONTIAC & SUNFIRE & $\mathrm{D}$ & $\mathrm{F}$ & L4 & 2.2 & G & M & 32.1 & 6.0 & 11.1 \\
\hline $\mathrm{C} 2$ & GMC - PONTIAC & SUNFIRE CNVT & $\mathrm{D}$ & $\mathrm{F}$ & L4 & 2.2 & G & A & 30.7 & 6.2 & 11.6 \\
\hline $\mathrm{C} 2$ & GMC - PONTIAC & SUNFIRE & $\mathrm{D}$ & $\mathrm{F}$ & L4 & 2.4 & G & M & 30.8 & 6.2 & 11.6 \\
\hline C3 & GMC - PONTIAC & GRAND AM & $\mathrm{D}$ & $\mathrm{F}$ & L4 & 2.4 & G & $\mathrm{M}$ & 29.5 & 6.5 & 12.1 \\
\hline C3 & GMC - PONTIAC & GRAND AM & $\mathrm{D}$ & $\mathrm{F}$ & L4 & 2.4 & G & A & 29.4 & 6.5 & 12.1 \\
\hline C4 & GMC - PONTIAC & GRAND PRIX 4Dr & $\mathrm{D}$ & $\mathrm{F}$ & V6 & 3.1 & G & A & 27.5 & 6.9 & 13.0 \\
\hline C4 & GMC - PONTIAC & GRAND PRIX & $\mathrm{D}$ & $\mathrm{F}$ & V6 & 3.8 & G & A & 26.9 & 7.1 & 13.3 \\
\hline C5 & GMC - PONTIAC & BONNEVILLE & D & $\mathrm{F}$ & V6 & 3.8 & G & A & 26.6 & 7.2 & 13.5 \\
\hline C3 & GMC - SATURN & SL & $\mathrm{D}$ & $\mathrm{F}$ & L4 & 1.9 & G & M & 38.7 & 5.0 & 9.2 \\
\hline $\mathrm{C} 2$ & GMC - SATURN & SC & $\mathrm{D}$ & $F$ & L4 & 1.9 & G & $M$ & 35.4 & 5.4 & 10.1 \\
\hline $\mathrm{C} 2$ & GMC - SATURN & SC & $\mathrm{D}$ & $\mathrm{F}$ & L4 & 1.9 & G & M & 38.1 & 5.0 & 9.4 \\
\hline C3 & GMC - SATURN & $\mathrm{SL}$ & $\mathrm{D}$ & $\mathrm{F}$ & L4 & 1.9 & G & M & 36.1 & 5.3 & 9.9 \\
\hline $\mathrm{C} 7$ & GMC - SATURN & SW & $\mathrm{D}$ & $\mathrm{F}$ & L4 & 1.9 & G & M & 36.1 & 5.3 & 9.9 \\
\hline C3 & GMC - SATURN & SL & $\mathrm{D}$ & $\mathrm{F}$ & L4 & 1.9 & G & $A$ & 34.4 & 5.6 & 10.4 \\
\hline $\mathrm{C} 2$ & GMC - SATURN & SC & D & $\mathrm{F}$ & L4 & 1.9 & G & A & 33.9 & 5.7 & 10.5 \\
\hline $\mathrm{C} 7$ & GMC - SATURN & SW & $\mathrm{D}$ & $\mathrm{F}$ & L4 & 1.9 & G & A & 35.4 & 5.4 & 10.1 \\
\hline $\mathrm{C} 2$ & GMC - SATURN & SC & $\mathrm{D}$ & $\mathrm{F}$ & L4 & 1.9 & G & A & 36.1 & 5.3 & 9.9 \\
\hline C3 & GMC - SATURN & SL & $\mathrm{D}$ & $\mathrm{F}$ & L4 & 1.9 & G & A & 36.0 & 5.3 & 9.9 \\
\hline $\mathrm{C7}$ & GMC - SATURN & SW & $\mathrm{D}$ & $\mathrm{F}$ & L4 & 1.9 & G & $A$ & 33.9 & 5.7 & 10.5 \\
\hline C4 & GMC - SATURN & LS & $\mathrm{D}$ & $\mathrm{F}$ & L4 & 2.2 & G & M & 31.9 & 6.0 & 11.2 \\
\hline C4 & GMC - SATURN & LS & $\mathrm{D}$ & $\mathrm{F}$ & L4 & 2.2 & G & A & 30.5 & 6.3 & 11.7 \\
\hline C8 & GMC - SATURN & LW & $\mathrm{D}$ & $\mathrm{F}$ & L4 & 2.2 & G & A & 29.4 & 6.5 & 12.1 \\
\hline C8 & GMC - SATURN & LW & D & $\mathrm{F}$ & V6 & 3.0 & G & A & 26.2 & 7.3 & 13.6 \\
\hline $\mathrm{C} 2$ & HONDA - ACURA & ACURA INTEGRA & 1 & $\mathrm{~F}$ & $\mathrm{~L} 4$ & 1.8 & G & $\mathrm{M}$ & 32.3 & 5.9 & 11.0 \\
\hline $\mathrm{C} 2$ & HONDA - ACURA & ACURA INTEGRA -R & I & $\mathrm{F}$ & L4 & 1.8 & G & M & 31.9 & 6.0 & 11.2 \\
\hline C6 & HONDA & INSIGHT & I & $\mathrm{F}$ & L3 & 1.0 & $\mathrm{G} / \mathrm{EL}$ & M & 76.3 & 2.6 & 4.7 \\
\hline $\mathrm{C} 2$ & HONDA & CIVIC HX & I & $\mathrm{F}$ & L4 & 1.6 & G & $\mathrm{M}$ & 44.7 & 4.3 & 8.0 \\
\hline $\mathrm{C} 2$ & HONDA & CIVIC HX & I & $\mathrm{F}$ & L4 & 1.6 & G & $A$ & 41.9 & 4.6 & 8.5 \\
\hline $\mathrm{C} 2$ & HONDA & CIVIC dx 4Dr & I & $\mathrm{F}$ & L4 & 1.6 & G & M & 37.2 & 5.2 & 9.6 \\
\hline $\mathrm{C} 2$ & HONDA & CIVIC ex & I & $\mathrm{F}$ & L4 & 1.6 & G & M & 37.2 & 5.2 & 9.6 \\
\hline $\mathrm{C} 2$ & HONDA & CIVIC vp & 1 & $\mathrm{~F}$ & L4 & 1.6 & G & A & 35.5 & 5.4 & 10.1 \\
\hline $\mathrm{C} 2$ & HONDA & CIVIC dx 4Dr & I & $\mathrm{F}$ & L4 & 1.6 & G & A & 35.6 & 5.4 & 10.0 \\
\hline C2 & HONDA & CIVIC ex & 1 & $\mathrm{~F}$ & L4 & 1.6 & G & A & 35.5 & 5.4 & 10.1 \\
\hline C2 & HONDA & CIVIC si & 1 & $\mathrm{~F}$ & L4 & 1.6 & G & $\mathrm{M}$ & 32.8 & 5.8 & 10.9 \\
\hline C4 & HONDA & ACCORD Ix & 1 & $\mathrm{~F}$ & L4 & 2.3 & G & $\mathrm{M}$ & 32.1 & 6.0 & 11.1 \\
\hline C4 & HONDA & ACCORD & I & $\mathrm{F}$ & L4 & 2.3 & G & $\mathrm{M}$ & 30.4 & 6.3 & 11.8 \\
\hline C4 & HONDA & ACCORD Ix & I & $\mathrm{F}$ & L4 & 2.3 & G & A & 29.9 & 6.4 & 11.9 \\
\hline C3 & HYUNDAI & ACCENT & I & $\mathrm{F}$ & L4 & 1.5 & G & $\mathrm{M}$ & 36.6 & 5.3 & 9.8 \\
\hline
\end{tabular}




\begin{tabular}{|c|c|c|c|c|c|c|c|c|c|c|c|}
\hline $\begin{array}{l}\text { Type-Size } \\
\text { Class }\end{array}$ & Manufacturer/Division & Name & $\begin{array}{l}\text { Dom I } \\
\text { Imp }\end{array}$ & DR & Cyl & Disp & Fuel & Trans & MPG & GHG & $\begin{array}{l}\text { Oil } \\
\text { Use }\end{array}$ \\
\hline$\overline{\mathrm{C} 2}$ & MITSUBISHI & MIRAGE 2D & $\mathrm{I}$ & $\bar{F}$ & $\overline{L 4}$ & 1.5 & $\bar{G}$ & $\bar{M}$ & $\overline{41.7}$ & 4.6 & $\overline{8.6}$ \\
\hline $\mathrm{C} 2$ & MITSUBISHI & MIRAGE & I & $\mathrm{F}$ & L4 & 1.8 & G & M & 36.3 & 5.3 & 9.8 \\
\hline $\mathrm{C} 2$ & MITSUBISHI & MIRAGE 2D & I & $\mathrm{F}$ & L4 & 1.5 & G & $A$ & 36.7 & 5.2 & 9.7 \\
\hline $\mathrm{C} 2$ & MITSUBISHI & MIRAGE & I & $\mathrm{F}$ & L4 & 1.8 & G & $A$ & 33.2 & 5.8 & 10.8 \\
\hline $\mathrm{C} 3$ & NISSAN & SENTRA & I & $\mathrm{F}$ & L4 & 1.8 & G & M & 34.9 & 5.5 & 10.2 \\
\hline $\mathrm{C} 3$ & NISSAN & SENTRA & I & $\mathrm{F}$ & L4 & 1.8 & G & A & 33.9 & 5.6 & 10.5 \\
\hline $\mathrm{C} 3$ & NISSAN & ALTIMA & I & $\mathrm{F}$ & L4 & 2.4 & G & M & 30.9 & 6.2 & 11.6 \\
\hline $\mathrm{C} 2$ & SUZUKI & SWIFT & I & $\mathrm{F}$ & L4 & 1.3 & G & M & 45.5 & 4.2 & 7.8 \\
\hline $\mathrm{C} 2$ & SUZUKI & ESTEEM & I & $\mathrm{F}$ & L4 & 1.6 & G & M & 38.6 & 5.0 & 9.3 \\
\hline $\mathrm{C} 2$ & SUZUKI & SWIFT & I & $\mathrm{F}$ & L4 & 1.3 & G & A & 37.6 & 5.1 & 9.5 \\
\hline $\mathrm{C} 2$ & SUZUKI & ESTEEM & I & $\mathrm{F}$ & L4 & 1.8 & G & M & 36.2 & 5.3 & 9.9 \\
\hline $\mathrm{C7}$ & SUZUKI & ESTEEM WAGON & I & $\mathrm{F}$ & L4 & 1.8 & G & M & 35.1 & 5.5 & 10.2 \\
\hline $\mathrm{C} 2$ & SUZUKI & ESTEEM & I & $\mathrm{F}$ & L4 & 1.6 & G & A & 35.0 & 5.5 & 10.2 \\
\hline $\mathrm{C7}$ & SUZUKI & ESTEEM WAGON & I & $\mathrm{F}$ & L4 & 1.6 & G & A & 33.7 & 5.7 & 10.6 \\
\hline $\mathrm{C} 7$ & SUZUKI & ESTEEM WAGON & I & $\mathrm{F}$ & L4 & 1.8 & G & A & 33.7 & 5.7 & 10.6 \\
\hline $\mathrm{C} 2$ & SUZUKI & ESTEEM & I & $\mathrm{F}$ & L4 & 1.8 & $\mathrm{G}$ & $A$ & 33.7 & 5.7 & 10.6 \\
\hline C4 & TOYOTA & CAMRY CNG & I & $\mathrm{F}$ & L4 & 2.2 & NG & $A$ & 30.2 & 5.4 & 1.8 \\
\hline $\mathrm{C} 3$ & TOYOTA & $\mathrm{ECHO}$ & I & $\mathrm{F}$ & L4 & 1.5 & G & M & 43.1 & 4.5 & 8.3 \\
\hline $\mathrm{C} 3$ & TOYOTA & COROLLA & I & $\mathrm{F}$ & L4 & 1.8 & G & M & 39.6 & 4.9 & 9.0 \\
\hline $\mathrm{C} 3$ & TOYOTA & $\mathrm{ECHO}$ & I & $\mathrm{F}$ & L4 & 1.5 & G & A & 39.4 & 4.9 & 9.1 \\
\hline $\mathrm{C} 3$ & TOYOTA & COROLLA & I & $\mathrm{F}$ & L4 & 1.8 & G & $A$ & 37.4 & 5.1 & 9.6 \\
\hline $\mathrm{C} 2$ & TOYOTA & CELICA & I & $\mathrm{F}$ & L4 & 1.8 & G & M & 35.2 & 5.4 & 10.1 \\
\hline $\mathrm{C} 2$ & TOYOTA & CELICA & I & $\mathrm{F}$ & L4 & 1.8 & G & A & 34.9 & 5.5 & 10.2 \\
\hline C3 & TOYOTA & COROLLA & I & $\mathrm{F}$ & L4 & 1.8 & G & A & 34.9 & 5.5 & 10.2 \\
\hline C3 & TOYOTA & CAMRY SOLARA & 1 & $\mathrm{~F}$ & L4 & 2.2 & G & M & 31.0 & 6.2 & 11.5 \\
\hline C4 & TOYOTA & CAMRY & 1 & $\mathrm{~F}$ & L4 & 2.2 & G & M & 31.0 & 6.2 & 11.5 \\
\hline $\mathrm{C} 3$ & TOYOTA & CAMRY SOLARA & I & $\mathrm{F}$ & L4 & 2.2 & G & A & 30.1 & 6.4 & 11.9 \\
\hline $\mathrm{C} 4$ & TOYOTA & CAMRY & I & $\mathrm{F}$ & L4 & 2.2 & G & A & 30.1 & 6.4 & 11.9 \\
\hline $\mathrm{C} 2$ & TOYOTA & $\begin{array}{l}\text { CAMRY SOLARA } \\
\text { CONVERT }\end{array}$ & I & $\mathrm{F}$ & L4 & 2.2 & G & $A$ & 30.1 & 6.4 & 11.9 \\
\hline C5 & TOYOTA & AVALON & I & $\mathrm{F}$ & V6 & 3.0 & G & A & 28.1 & 6.8 & 12.7 \\
\hline $\mathrm{C} 3$ & VWA - AUDI & AUDI A4 & I & $\mathrm{F}$ & L4 & 1.8 & G & M & 31.9 & 6.0 & 11.2 \\
\hline $\mathrm{C} 2$ & VWA & NEW BEETLE & I & $\mathrm{F}$ & L4 & 1.9 & $D$ & M & 52.7 & 4.0 & 6.8 \\
\hline $\mathrm{C} 3$ & VWA & GOLF TDI & I & $\mathrm{F}$ & L4 & 1.9 & $D$ & M & 52.7 & 4.0 & 6.8 \\
\hline $\mathrm{C} 3$ & VWA & JETTA & I & $\mathrm{F}$ & L4 & 1.9 & $D$ & M & 52.7 & 4.0 & 6.8 \\
\hline $\mathrm{C} 2$ & VWA & NEW BEETLE & I & $\mathrm{F}$ & L4 & 1.9 & $D$ & $A$ & 44.7 & 4.7 & 8.0 \\
\hline $\mathrm{C} 3$ & VWA & GOLF TDI & I & $\mathrm{F}$ & L4 & 1.9 & $D$ & A & 44.7 & 4.7 & 8.0 \\
\hline C3 & VWA & JETTA & I & $\mathrm{F}$ & L4 & 1.9 & $D$ & A & 44.7 & 4.7 & 8.0 \\
\hline $\mathrm{C} 2$ & VWA & NEW BEETLE & I & $\mathrm{F}$ & L4 & 1.8 & G & M & 32.3 & 5.9 & 11.1 \\
\hline $\mathrm{C} 4$ & VWA & PASSAT & 1 & $\mathrm{~F}$ & L4 & 1.8 & G & M & 31.3 & 6.1 & 11.4 \\
\hline $\mathrm{C} 8$ & VWA & PASSAT WAGON & I & $\mathrm{F}$ & L4 & 1.8 & $\mathrm{G}$ & M & 31.3 & 6.1 & 11.4 \\
\hline T3 & DCC - CHRYSLER & $\begin{array}{l}\text { TOWN \& CNTRY 2WD } \\
\text { FFV }\end{array}$ & D & $\mathrm{F}$ & V6 & 3.3 & FF & $A$ & 20.0 & 7.0 & 9.1 \\
\hline T4 & DCC - DODGE & B2500 WAGON & D & $\mathrm{R}$ & V8 & 5.2 & NG & A & 15.2 & 10.2 & 3.5 \\
\hline $\mathrm{T} 4$ & DCC - DODGE & B2500 VAN & $D$ & $\mathrm{R}$ & V8 & 5.2 & NG & $A$ & 15.5 & 10.0 & 3.5 \\
\hline T3 & DCC - DODGE & CARAVAN 2WD FFV & $D$ & $\mathrm{~F}$ & V6 & 3.3 & FF & A & 19.9 & 7.1 & 9.2 \\
\hline T3 & DCC - DODGE & CARAVAN 2WD & $\mathrm{D}$ & $\mathrm{F}$ & L4 & 2.4 & G & $A$ & 26.0 & 7.3 & 13.7 \\
\hline T3 & DCC - PLYMOUTH & $\begin{array}{l}\text { CARAVAN/VOYAGER } \\
\text { 2WD }\end{array}$ & $\mathrm{D}$ & $\mathrm{F}$ & L4 & 2.4 & G & A & 25.9 & 7.3 & 13.8 \\
\hline T3 & DCC - PLYMOUTH & $\begin{array}{l}\text { CARAVAN/VOYAGER } \\
\text { 2WD }\end{array}$ & $\mathrm{D}$ & $\mathrm{F}$ & V6 & 3.3 & FF & A & 19.9 & 7.1 & 9.2 \\
\hline T1 & FMC - FORD & RANGER 4X2 EV & $\mathrm{D}$ & $\mathrm{R}$ & E0 & 2.5 & EL & A & 29.7 & 5.2 & 1.8 \\
\hline
\end{tabular}




\begin{tabular}{|c|c|c|c|c|c|c|c|c|c|c|c|}
\hline $\begin{array}{l}\text { Type-Size } \\
\text { Class }\end{array}$ & Manufacturer/Division & Name & $\begin{array}{l}\text { Dom I } \\
\text { Imp }\end{array}$ & DR & Cyl & Disp & Fuel & Trans & MPG & GHG & $\begin{array}{l}\text { Oil } \\
\text { Use }\end{array}$ \\
\hline$\overline{\mathrm{T} 2}$ & FMC - FORD & F150 4X2 NGV & $\mathrm{D}$ & $\bar{R}$ & V8 & 5.4 & NG & $\bar{A}$ & 15.8 & 9.9 & 3.4 \\
\hline T4 & FMC - FORD & E250 VAN NGV & $D$ & $\mathrm{R}$ & V8 & 5.4 & NG & $A$ & 15.5 & 10.1 & 3.5 \\
\hline T1 & FMC - FORD & RANGER 4X2 FFV & $D$ & $\mathrm{R}$ & V6 & 3.0 & FF & M & 19.7 & 7.1 & 9.2 \\
\hline T1 & FMC - FORD & RANGER 4X4 FFV & $\mathrm{D}$ & 4 & V6 & 3.0 & FF & M & 19.2 & 7.3 & 9.5 \\
\hline $\mathrm{T} 1$ & FMC - FORD & RANGER 4X2 FFV & $\mathrm{D}$ & $\mathrm{R}$ & V6 & 3.0 & FF & A & 18.5 & 7.6 & 9.7 \\
\hline $\mathrm{T} 1$ & FMC - FORD & RANGER 4X4 FFV & $\mathrm{D}$ & 4 & V6 & 3.0 & FF & $A$ & 17.7 & 8.0 & 10.4 \\
\hline T1 & FMC - FORD & RANGER 4X2 & $D$ & $\mathrm{R}$ & L4 & 2.5 & G & M & 28.3 & 6.7 & 12.6 \\
\hline T1 & GMC - CHEVROLET & S10 P/U 2WD & $D$ & $\mathrm{R}$ & L4 & 2.2 & FF & M & 23.9 & 5.9 & 7.6 \\
\hline T1 & GMC - CHEVROLET & S10 P/U 2WD & $\mathrm{D}$ & $\mathrm{R}$ & L4 & 2.2 & FF & $A$ & 21.2 & 6.7 & 8.7 \\
\hline T1 & GMC - CHEVROLET & S10 P/U 2WD & $D$ & $\mathrm{R}$ & L4 & 2.2 & G & M & 29.6 & 6.5 & 12.1 \\
\hline T1 & GMC & SONOMA 2WD & I & $\mathrm{R}$ & L4 & 2.2 & FF & M & 23.9 & 5.9 & 7.6 \\
\hline T1 & GMC & SONOMA 2WD & I & $\mathrm{R}$ & L4 & 2.2 & FF & $A$ & 21.2 & 6.7 & 8.7 \\
\hline T1 & GMC & SONOMA 2WD & I & $\mathrm{R}$ & L4 & 2.2 & G & M & 29.6 & 6.5 & 12.1 \\
\hline T3 & GMC - OLDSMOBILE & SILHOUETTE 2WD & $\mathrm{D}$ & $\mathrm{F}$ & V6 & 3.4 & G & A & 25.0 & 7.6 & 14.3 \\
\hline T3 & HONDA & ODYSSEY & I & $\mathrm{F}$ & V6 & 3.5 & G & A & 24.0 & 7.9 & 14.9 \\
\hline T1 & ISUZU & HOMBRE P/U 2WD & I & $\mathrm{R}$ & L4 & 2.2 & FF & M & 23.9 & 5.9 & 7.6 \\
\hline $\mathrm{T} 1$ & ISUZU & HOMBRE P/U 2WD & I & $\mathrm{R}$ & L4 & 2.2 & FF & $A$ & 21.2 & 6.7 & 8.7 \\
\hline T1 & TKM - MAZDA & MAZDA 4X2 FFV & I & $\mathrm{R}$ & V6 & 3.0 & FF & M & 19.7 & 7.1 & 9.2 \\
\hline T1 & TKM - MAZDA & MAZDA 4X4 FFV & I & 4 & V6 & 3.0 & FF & M & 19.2 & 7.3 & 9.5 \\
\hline T1 & TKM - MAZDA & MAZDA 4X2 FFV & I & $\mathrm{R}$ & V6 & 3.0 & FF & A & 18.5 & 7.6 & 9.7 \\
\hline $\mathrm{T} 1$ & TKM - MAZDA & MAZDA 4X4 FFV & I & 4 & V6 & 3.0 & FF & $A$ & 17.7 & 8.0 & 10.4 \\
\hline T1 & TKM - MAZDA & MAZDA 4X2 & I & $\mathrm{R}$ & L4 & 2.5 & G & $M$ & 28.3 & 6.7 & 12.6 \\
\hline
\end{tabular}


Energy Star Method 13 Vehicle List

Top $20 \%$ of vehicles by GHG and oil use per footprint [not normalized]

\begin{tabular}{|c|c|c|c|c|c|c|c|c|c|c|c|}
\hline $\begin{array}{l}\text { Type-Size } \\
\text { Class } \\
\end{array}$ & Manufacturer/Division & Name & $\begin{array}{c}\text { Dom I } \\
\text { Imp }\end{array}$ & DR & Cyl & Disp & Fuel & Trans & MPG & GHG & $\begin{array}{l}\text { Oil } \\
\text { Use }\end{array}$ \\
\hline \multicolumn{12}{|l|}{ Cars } \\
\hline $\mathrm{C} 4$ & DAEWOO & LANOS- 3Dr & I & $\mathrm{F}$ & $\mathrm{L} 4$ & 1.5 & $G$ & $M$ & 34.8 & 5.5 & 10.3 \\
\hline C4 & DAEWOO & LANOS-3Dr & I & $\mathrm{F}$ & $\mathrm{L} 4$ & 1.6 & G & M & 34.6 & 5.5 & 10.3 \\
\hline C4 & DAEWOO & LANOS- 3Dr & I & $\mathrm{F}$ & $\mathrm{L} 4$ & 1.5 & G & A & 32.9 & 5.8 & 10.8 \\
\hline C3 & DCC - CHRYSLER & NEON & $\mathrm{D}$ & $\mathrm{F}$ & $\mathrm{L} 4$ & 2.0 & G & M & 36.1 & 5.3 & 9.9 \\
\hline C4 & DCC - CHRYSLER & CIRRUS & $\mathrm{D}$ & $\mathrm{F}$ & $\mathrm{L} 4$ & 2.0 & G & M & 34.3 & 5.6 & 10.4 \\
\hline C3 & DCC - CHRYSLER & NEON & $\mathrm{D}$ & $\mathrm{F}$ & L4 & 2.0 & G & $A$ & 31.9 & 6.0 & 11.2 \\
\hline C5 & DCC - CHRYSLER & INTREPID & $\mathrm{D}$ & $\mathrm{F}$ & V6 & 2.7 & G & A & 27.3 & 7.0 & 13.1 \\
\hline C5 & DCC - CHRYSLER & CONCORDE & $\mathrm{D}$ & $\mathrm{F}$ & V6 & 2.7 & G & A & 27.3 & 7.0 & 13.1 \\
\hline C5 & DCC - CHRYSLER & INTREPID & $\mathrm{D}$ & $\mathrm{F}$ & V6 & 3.2 & G & $A$ & 25.5 & 7.5 & 14.0 \\
\hline C3 & DCC - DODGE & NEON & $\mathrm{D}$ & $\mathrm{F}$ & $\mathrm{L} 4$ & 2.0 & G & $M$ & 36.1 & 5.3 & 9.9 \\
\hline $\mathrm{C} 4$ & DCC - DODGE & STRATUS & $\mathrm{D}$ & $\mathrm{F}$ & $\mathrm{L} 4$ & 2.0 & G & M & 28.3 & 6.7 & 12.6 \\
\hline C3 & DCC - DODGE & NEON & $\mathrm{D}$ & $\mathrm{F}$ & L4 & 2.0 & G & A & 31.9 & 6.0 & 11.2 \\
\hline C4 & DCC - DODGE & STRATUS & $\mathrm{D}$ & $\mathrm{F}$ & $\mathrm{L} 4$ & 2.0 & G & $\mathrm{M}$ & 28.3 & 6.7 & 12.6 \\
\hline C5 & DCC - DODGE & INTREPID & $\mathrm{D}$ & $\mathrm{F}$ & V6 & 2.7 & G & A & 27.3 & 7.0 & 13.1 \\
\hline C5 & DCC - DODGE & INTREPID & $\mathrm{D}$ & $\mathrm{F}$ & V6 & 2.7 & G & A & 27.3 & 7.0 & 13.1 \\
\hline C5 & DCC - DODGE & INTREPID & $\mathrm{D}$ & $\mathrm{F}$ & V6 & 3.2 & G & A & 25.5 & 7.5 & 14.0 \\
\hline C4 & DCC - MER-BENZ & E 320 & I & $\mathrm{R}$ & V6 & 3.2 & G & A & 27.9 & 6.8 & 12.8 \\
\hline C3 & DCC - PLYMOUTH & NEON & $\mathrm{D}$ & $\mathrm{F}$ & $\mathrm{L} 4$ & 2.0 & G & $M$ & 36.1 & 5.3 & 9.9 \\
\hline $\mathrm{C} 4$ & DCC - PLYMOUTH & BREEZE & $\mathrm{D}$ & $\mathrm{F}$ & $\mathrm{L} 4$ & 2.0 & G & $M$ & 35.4 & 5.4 & 10.1 \\
\hline C3 & DCC - PLYMOUTH & NEON & $\mathrm{D}$ & $\mathrm{F}$ & $\mathrm{L} 4$ & 2.0 & G & A & 31.9 & 6.0 & 11.2 \\
\hline C4 & DCC - PLYMOUTH & BREEZE & $\mathrm{D}$ & $\mathrm{F}$ & L4 & 2.0 & G & $A$ & 28.3 & 6.7 & 12.6 \\
\hline C5 & FMC - FORD & CROWN VICTORIA NG & $\mathrm{D}$ & $\mathrm{R}$ & V8 & 4.6 & NG & $A$ & 20.7 & 7.6 & 2.6 \\
\hline C5 & FMC - FORD & TAURUS FFV & $\mathrm{D}$ & $\mathrm{F}$ & V6 & 3.0 & $\mathrm{FF}$ & A & 24.6 & 5.9 & 7.8 \\
\hline C8 & FMC - FORD & TAURUS WAGON FFV & $\mathrm{D}$ & $\mathrm{F}$ & V6 & 3.0 & $\mathrm{FF}$ & $A$ & 23.3 & 6.2 & 8.3 \\
\hline $\mathrm{C7}$ & FMC - FORD & FOCUS WAGON & $\mathrm{D}$ & $\mathrm{F}$ & $\mathrm{L} 4$ & 2.0 & G & M & 36.2 & 5.3 & 9.9 \\
\hline C3 & FMC - FORD & FOCUS & $\mathrm{D}$ & $\mathrm{F}$ & L4 & 2.0 & G & M & 35.7 & 5.4 & 10.0 \\
\hline C3 & FMC - FORD & ESCORT & $\mathrm{D}$ & $\mathrm{F}$ & $\mathrm{L} 4$ & 2.0 & G & $\mathrm{M}$ & 35.7 & 5.4 & 10.0 \\
\hline C3 & FMC - FORD & FOCUS & $\mathrm{D}$ & $\mathrm{F}$ & $\mathrm{L} 4$ & 2.0 & G & A & 33.9 & 5.7 & 10.5 \\
\hline $\mathrm{C7}$ & FMC - FORD & FOCUS WAGON & $\mathrm{D}$ & $\mathrm{F}$ & $\mathrm{L} 4$ & 2.0 & G & A & 32.6 & 5.9 & 11.0 \\
\hline C3 & FMC - FORD & ESCORT & $\mathrm{D}$ & $\mathrm{F}$ & $\mathrm{L} 4$ & 2.0 & G & $A$ & 33.7 & 5.7 & 10.6 \\
\hline $\mathrm{C} 2$ & FMC - FORD & ESCORT ZX2 & $\mathrm{D}$ & $\mathrm{F}$ & L4 & 2.0 & G & M & 33.0 & 5.8 & 10.8 \\
\hline C3 & FMC - FORD & FOCUS 3D & $\mathrm{D}$ & $\mathrm{F}$ & $\mathrm{L} 4$ & 2.0 & G & $M$ & 33.0 & 5.8 & 10.8 \\
\hline C3 & FMC - FORD & FOCUS 3D & $\mathrm{D}$ & $\mathrm{F}$ & $\mathrm{L} 4$ & 2.0 & G & $A$ & 32.4 & 5.9 & 11.0 \\
\hline $\mathrm{C} 2$ & FMC - FORD & ESCORT ZX2 & $\mathrm{D}$ & $\mathrm{F}$ & $\mathrm{L} 4$ & 2.0 & G & $A$ & 33.1 & 5.8 & 10.8 \\
\hline $\mathrm{C7}$ & FMC - FORD & FOCUS WAGON & $\mathrm{D}$ & $\mathrm{F}$ & L4 & 2.0 & G & $A$ & 32.6 & 5.9 & 11.0 \\
\hline C3 & FMC - FORD & CONTOUR & $\mathrm{D}$ & $\mathrm{F}$ & $\mathrm{L} 4$ & 2.0 & G & $M$ & 32.6 & 5.9 & 11.0 \\
\hline C3 & FMC - FORD & CONTOUR & $\mathrm{D}$ & $F$ & $\mathrm{~L} 4$ & 2.0 & G & $A$ & 30.3 & 6.3 & 11.8 \\
\hline C3 & FMC - FORD & CONTOUR & $\mathrm{D}$ & $\mathrm{F}$ & L4 & 2.0 & G & A & 30.3 & 6.3 & 11.8 \\
\hline C3 & FMC - MAZDA & PROTEGE & I & $\mathrm{F}$ & $\mathrm{L} 4$ & 1.6 & G & $A$ & 33.8 & 5.7 & 10.6 \\
\hline C4 & FMC - MAZDA & 626 & I & $\mathrm{F}$ & $\mathrm{L} 4$ & 2.0 & G & M & 33.5 & 5.7 & 10.6 \\
\hline C3 & FMC - MAZDA & PROTEGE & 1 & $\mathrm{~F}$ & L4 & 1.8 & G & $\mathrm{M}$ & 32.4 & 5.9 & 11.0 \\
\hline C3 & FMC - MAZDA & PROTEGE & I & $\mathrm{F}$ & L4 & 1.6 & G & $M$ & 36.5 & 5.3 & 9.8 \\
\hline C3 & FMC - MERCURY & MYSTIQUE & $\mathrm{D}$ & $\mathrm{F}$ & $\mathrm{L} 4$ & 2.0 & G & M & 32.4 & 5.9 & 11.0 \\
\hline C3 & FMC - MERCURY & COUGAR & $\mathrm{D}$ & $\mathrm{F}$ & $\mathrm{L} 4$ & 2.0 & G & M & 32.4 & 5.9 & 11.0 \\
\hline C3 & FMC - MERCURY & MYSTIQUE & $D$ & $\mathrm{~F}$ & L4 & 2.0 & G & $A$ & 29.6 & 6.5 & 12.1 \\
\hline
\end{tabular}




\begin{tabular}{|c|c|c|c|c|c|c|c|c|c|c|c|}
\hline $\begin{array}{l}\text { Type-Size } \\
\text { Class }\end{array}$ & Manufacturer/Division & Name & $\begin{array}{c}\text { Dom I } \\
\text { Imp }\end{array}$ & DR & Cyl & Disp & Fuel & Trans & MPG & GHG & $\begin{array}{l}\text { Oil } \\
\text { Use }\end{array}$ \\
\hline C5 & GMC - BUICK & LESABRE & D & $\mathrm{F}$ & V6 & 3.8 & G & A & 26.6 & 7.2 & 13.5 \\
\hline C5 & GMC - BUICK & PARK AVENUE & D & $\mathrm{F}$ & V6 & 3.8 & G & A & 26.6 & 7.2 & 13.5 \\
\hline C2 & GMC - CHEVY & METRO & 1 & $\mathrm{~F}$ & L3 & 1.0 & G & M & 48.9 & 4.0 & 7.3 \\
\hline C2 & GMC - CHEVY & METRO & 1 & $\mathrm{~F}$ & L4 & 1.3 & G & M & 45.5 & 4.2 & 7.8 \\
\hline C3 & GMC - CHEVY & PRIZM & 1 & $\mathrm{~F}$ & L4 & 1.8 & G & M & 39.6 & 4.9 & 9.0 \\
\hline C3 & GMC - CHEVY & PRIZM & 1 & $\mathrm{~F}$ & L4 & 1.8 & G & A & 37.5 & 5.1 & 9.5 \\
\hline C2 & GMC - CHEVY & METRO & 1 & $\mathrm{~F}$ & L4 & 1.3 & G & A & 37.6 & 5.1 & 9.5 \\
\hline C3 & GMC - CHEVY & PRIZM & 1 & $\mathrm{~F}$ & L4 & 1.8 & G & A & 34.9 & 5.5 & 10.2 \\
\hline C2 & GMC - CHEVY & CAVALIER & D & $\mathrm{F}$ & L4 & 2.2 & G & M & 32.1 & 6.0 & 11.1 \\
\hline C2 & GMC - CHEVY & CAVALIER Z24 & D & $\mathrm{F}$ & L4 & 2.4 & G & M & 30.8 & 6.2 & 11.6 \\
\hline C5 & GMC - CHEVY & IMPALA & D & $\mathrm{F}$ & V6 & 3.4 & G & A & 27.5 & 6.9 & 13.0 \\
\hline C5 & GMC - CHEVY & IMPALA & D & $\mathrm{F}$ & V6 & 3.8 & G & A & 26.9 & 7.1 & 13.3 \\
\hline C3 & GMC - OLDSMOBILE & ALERO & D & $\mathrm{F}$ & L4 & 2.4 & G & M & 29.5 & 6.5 & 12.1 \\
\hline C3 & GMC - OLDSMOBILE & ALERO & $\mathrm{D}$ & $\mathrm{F}$ & L4 & 2.4 & G & A & 29.4 & 6.5 & 12.1 \\
\hline C2 & GMC - PONTIAC & SUNFIRE & D & $\mathrm{F}$ & L4 & 2.2 & G & M & 32.1 & 6.0 & 11.1 \\
\hline C2 & GMC - PONTIAC & SUNFIRE CNVT & D & $\mathrm{F}$ & L4 & 2.2 & G & A & 30.7 & 6.2 & 11.6 \\
\hline C2 & GMC - PONTIAC & SUNFIRE & D & $\mathrm{F}$ & L4 & 2.4 & G & M & 30.8 & 6.2 & 11.6 \\
\hline C3 & GMC - PONTIAC & GRAND AM & D & $\mathrm{F}$ & L4 & 2.4 & G & M & 29.5 & 6.5 & 12.1 \\
\hline C3 & GMC - PONTIAC & GRAND AM & D & $\mathrm{F}$ & L4 & 2.4 & G & A & 29.4 & 6.5 & 12.1 \\
\hline C4 & GMC - PONTIAC & GRAND PRIX 4Dr & D & $\mathrm{F}$ & V6 & 3.1 & G & A & 27.5 & 6.9 & 13.0 \\
\hline C4 & GMC - PONTIAC & GRAND PRIX & $\mathrm{D}$ & $\mathrm{F}$ & V6 & 3.8 & G & A & 26.9 & 7.1 & 13.3 \\
\hline C5 & GMC - PONTIAC & BONNEVILLE & D & $\mathrm{F}$ & V6 & 3.8 & G & A & 26.6 & 7.2 & 13.5 \\
\hline C3 & GMC - SATURN & SL & D & $\mathrm{F}$ & L4 & 1.9 & G & M & 38.7 & 5.0 & 9.2 \\
\hline C2 & GMC - SATURN & SC & D & $\mathrm{F}$ & L4 & 1.9 & G & M & 35.4 & 5.4 & 10.1 \\
\hline C2 & GMC - SATURN & SC & D & $\mathrm{F}$ & L4 & 1.9 & G & M & 38.1 & 5.0 & 9.4 \\
\hline C3 & GMC - SATURN & SL & D & $\mathrm{F}$ & L4 & 1.9 & G & M & 36.1 & 5.3 & 9.9 \\
\hline C7 & GMC - SATURN & sW & D & $\mathrm{F}$ & L4 & 1.9 & G & M & 36.1 & 5.3 & 9.9 \\
\hline C3 & GMC - SATURN & SL & D & $\mathrm{F}$ & L4 & 1.9 & G & A & 34.4 & 5.6 & 10.4 \\
\hline C2 & GMC - SATURN & SC & D & $\mathrm{F}$ & L4 & 1.9 & G & A & 33.9 & 5.7 & 10.5 \\
\hline C7 & GMC - SATURN & SW & D & $\mathrm{F}$ & L4 & 1.9 & G & A & 35.4 & 5.4 & 10.1 \\
\hline C2 & GMC - SATURN & SC & D & $\mathrm{F}$ & L4 & 1.9 & G & A & 36.1 & 5.3 & 9.9 \\
\hline C3 & GMC - SATURN & SL & D & $\mathrm{F}$ & L4 & 1.9 & G & A & 36.0 & 5.3 & 9.9 \\
\hline C7 & GMC - SATURN & SW & D & $\mathrm{F}$ & L4 & 1.9 & G & A & 33.9 & 5.7 & 10.5 \\
\hline C4 & GMC - SATURN & LS & D & $\mathrm{F}$ & L4 & 2.2 & G & M & 31.9 & 6.0 & 11.2 \\
\hline C4 & GMC - SATURN & LS & D & $\mathrm{F}$ & L4 & 2.2 & G & A & 30.5 & 6.3 & 11.7 \\
\hline C8 & GMC - SATURN & LW & D & $\mathrm{F}$ & L4 & 2.2 & G & A & 29.4 & 6.5 & 12.1 \\
\hline C8 & GMC - SATURN & LW & D & $\mathrm{F}$ & V6 & 3.0 & G & A & 26.2 & 7.3 & 13.6 \\
\hline C2 & HONDA - ACURA & ACURA INTEGRA & 1 & $\mathrm{~F}$ & L4 & 1.8 & G & M & 32.3 & 5.9 & 11.0 \\
\hline C2 & HONDA - ACURA & ACURA INTEGRA -R & 1 & $\mathrm{~F}$ & L4 & 1.8 & G & M & 31.9 & 6.0 & 11.2 \\
\hline C6 & HONDA & INSIGHT & 1 & $\mathrm{~F}$ & L3 & 1.0 & G/EL & M & 76.3 & 2.6 & 4.7 \\
\hline C2 & HONDA & CIVIC HX & 1 & $\mathrm{~F}$ & L4 & 1.6 & G & M & 44.7 & 4.3 & 8.0 \\
\hline $\mathrm{C} 2$ & HONDA & CIVIC HX & 1 & $\mathrm{~F}$ & L4 & 1.6 & G & A & 41.9 & 4.6 & 8.5 \\
\hline C2 & HONDA & CIVIC dx 4Dr & 1 & $\mathrm{~F}$ & L4 & 1.6 & G & M & 37.2 & 5.2 & 9.6 \\
\hline C2 & HONDA & CIVIC ex & 1 & $\mathrm{~F}$ & L4 & 1.6 & G & M & 37.2 & 5.2 & 9.6 \\
\hline $\mathrm{C} 2$ & HONDA & CIVIC vp & 1 & $\mathrm{~F}$ & L4 & 1.6 & G & A & 35.5 & 5.4 & 10.1 \\
\hline $\mathrm{C} 2$ & HONDA & CIVIC dx 4Dr & 1 & $\mathrm{~F}$ & L4 & 1.6 & G & A & 35.6 & 5.4 & 10.0 \\
\hline C2 & HONDA & CIVIC ex & 1 & $\mathrm{~F}$ & L4 & 1.6 & G & A & 35.5 & 5.4 & 10.1 \\
\hline C2 & HONDA & CIVIC si & 1 & $\mathrm{~F}$ & L4 & 1.6 & G & M & 32.8 & 5.8 & 10.9 \\
\hline C4 & HONDA & ACCORD Ix & 1 & $\mathrm{~F}$ & L4 & 2.3 & G & M & 32.1 & 6.0 & 11.1 \\
\hline
\end{tabular}




\begin{tabular}{|c|c|c|c|c|c|c|c|c|c|c|c|}
\hline $\begin{array}{l}\text { Type-Size } \\
\text { Class }\end{array}$ & Manufacturer/Division & Name & $\begin{array}{l}\text { Dom II } \\
\text { Imp }\end{array}$ & DR & Cyl & Disp & Fuel & Trans & MPG & GHG & $\begin{array}{l}\text { Oil } \\
\text { Use }\end{array}$ \\
\hline C4 & HONDA & ACCORD & I & $\mathrm{F}$ & L4 & 2.3 & G & M & 30.4 & 6.3 & 11.8 \\
\hline C4 & HONDA & ACCORD Ix & 1 & $\mathrm{~F}$ & L4 & 2.3 & G & A & 29.9 & 6.4 & 11.9 \\
\hline C3 & HYUNDAI & ACCENT & 1 & $\mathrm{~F}$ & L4 & 1.5 & G & M & 36.6 & 5.3 & 9.8 \\
\hline C2 & MITSUBISHI & MIRAGE 2D & 1 & $\mathrm{~F}$ & L4 & 1.5 & G & M & 41.7 & 4.6 & 8.6 \\
\hline C2 & MITSUBISHI & MIRAGE & I & $\mathrm{F}$ & L4 & 1.8 & G & M & 36.3 & 5.3 & 9.8 \\
\hline C2 & MITSUBISHI & MIRAGE 2D & 1 & $\mathrm{~F}$ & L4 & 1.5 & G & A & 36.7 & 5.2 & 9.7 \\
\hline C2 & MITSUBISHI & MIRAGE & 1 & $\mathrm{~F}$ & L4 & 1.8 & G & A & 33.2 & 5.8 & 10.8 \\
\hline C3 & NISSAN & SENTRA & 1 & $\mathrm{~F}$ & L4 & 1.8 & G & M & 34.9 & 5.5 & 10.2 \\
\hline C3 & NISSAN & SENTRA & 1 & $\mathrm{~F}$ & L4 & 1.8 & G & A & 33.9 & 5.6 & 10.5 \\
\hline C3 & NISSAN & ALTIMA & 1 & $\mathrm{~F}$ & L4 & 2.4 & G & M & 30.9 & 6.2 & 11.6 \\
\hline C2 & SUZUKI & SWIFT & 1 & $\mathrm{~F}$ & L4 & 1.3 & G & M & 45.5 & 4.2 & 7.8 \\
\hline C2 & SUZUKI & ESTEEM & I & $\mathrm{F}$ & L4 & 1.6 & G & M & 38.6 & 5.0 & 9.3 \\
\hline C2 & SUZUKI & SWIFT & 1 & $\mathrm{~F}$ & L4 & 1.3 & G & A & 37.6 & 5.1 & 9.5 \\
\hline C2 & SUZUKI & ESTEEM & 1 & $\mathrm{~F}$ & L4 & 1.8 & G & M & 36.2 & 5.3 & 9.9 \\
\hline $\mathrm{C} 7$ & SUZUKI & ESTEEM WAGON & 1 & $\mathrm{~F}$ & L4 & 1.8 & G & M & 35.1 & 5.5 & 10.2 \\
\hline C2 & SUZUKI & ESTEEM & 1 & $\mathrm{~F}$ & L4 & 1.6 & G & A & 35.0 & 5.5 & 10.2 \\
\hline $\mathrm{C} 7$ & SUZUKI & ESTEEM WAGON & 1 & $\mathrm{~F}$ & L4 & 1.6 & G & A & 33.7 & 5.7 & 10.6 \\
\hline C7 & SUZUKI & ESTEEM WAGON & 1 & $\mathrm{~F}$ & L4 & 1.8 & G & A & 33.7 & 5.7 & 10.6 \\
\hline C2 & SUZUKI & ESTEEM & 1 & $\mathrm{~F}$ & L4 & 1.8 & G & A & 33.7 & 5.7 & 10.6 \\
\hline C4 & TOYOTA & CAMRY CNG & 1 & $\mathrm{~F}$ & L4 & 2.2 & NG & A & 30.2 & 5.4 & 1.8 \\
\hline C3 & TOYOTA & $\mathrm{ECHO}$ & I & $\mathrm{F}$ & L4 & 1.5 & G & M & 43.1 & 4.5 & 8.3 \\
\hline C3 & TOYOTA & COROLLA & 1 & $\mathrm{~F}$ & L4 & 1.8 & G & M & 39.6 & 4.9 & 9.0 \\
\hline C3 & TOYOTA & ECHO & 1 & $\mathrm{~F}$ & L4 & 1.5 & G & A & 39.4 & 4.9 & 9.1 \\
\hline C3 & TOYOTA & COROLLA & 1 & $\mathrm{~F}$ & L4 & 1.8 & G & A & 37.4 & 5.1 & 9.6 \\
\hline C2 & TOYOTA & CELICA & 1 & $\mathrm{~F}$ & L4 & 1.8 & G & M & 35.2 & 5.4 & 10.1 \\
\hline C2 & TOYOTA & CELICA & 1 & $\mathrm{~F}$ & L4 & 1.8 & G & A & 34.9 & 5.5 & 10.2 \\
\hline C3 & TOYOTA & COROLLA & 1 & $\mathrm{~F}$ & L4 & 1.8 & G & A & 34.9 & 5.5 & 10.2 \\
\hline C3 & TOYOTA & CAMRY SOLARA & I & $\mathrm{F}$ & L4 & 2.2 & G & M & 31.0 & 6.2 & 11.5 \\
\hline C4 & TOYOTA & CAMRY & 1 & $\mathrm{~F}$ & L4 & 2.2 & G & M & 31.0 & 6.2 & 11.5 \\
\hline C3 & TOYOTA & CAMRY SOLARA & 1 & $\mathrm{~F}$ & L4 & 2.2 & G & A & 30.1 & 6.4 & 11.9 \\
\hline C4 & TOYOTA & CAMRY & 1 & $\mathrm{~F}$ & L4 & 2.2 & G & A & 30.1 & 6.4 & 11.9 \\
\hline $\mathrm{C} 2$ & TOYOTA & $\begin{array}{l}\text { CAMRY SOLARA } \\
\text { CONVERT }\end{array}$ & I & $\mathrm{F}$ & L4 & 2.2 & G & A & 30.1 & 6.4 & 11.9 \\
\hline C5 & TOYOTA & AVALON & 1 & $\mathrm{~F}$ & V6 & 3.0 & G & A & 28.1 & 6.8 & 12.7 \\
\hline C3 & VWA - AUDI & AUDI A4 & 1 & $\mathrm{~F}$ & L4 & 1.8 & G & M & 31.9 & 6.0 & 11.2 \\
\hline C2 & VWA & NEW BEETLE & 1 & $\mathrm{~F}$ & L4 & 1.9 & D & M & 52.7 & 4.0 & 6.8 \\
\hline C3 & VWA & GOLF TDI & 1 & $\mathrm{~F}$ & L4 & 1.9 & D & M & 52.7 & 4.0 & 6.8 \\
\hline C3 & VWA & JETTA & 1 & $\mathrm{~F}$ & L4 & 1.9 & D & M & 52.7 & 4.0 & 6.8 \\
\hline C2 & VWA & NEW BEETLE & I & $\mathrm{F}$ & L4 & 1.9 & D & A & 44.7 & 4.7 & 8.0 \\
\hline C3 & VWA & GOLF TDI & 1 & $\mathrm{~F}$ & L4 & 1.9 & D & A & 44.7 & 4.7 & 8.0 \\
\hline C3 & VWA & JETTA & 1 & $\mathrm{~F}$ & L4 & 1.9 & D & A & 44.7 & 4.7 & 8.0 \\
\hline C2 & VWA & NEW BEETLE & 1 & $\mathrm{~F}$ & L4 & 1.8 & G & M & 32.3 & 5.9 & 11.1 \\
\hline C4 & VWA & PASSAT & 1 & $\mathrm{~F}$ & L4 & 1.8 & G & M & 31.3 & 6.1 & 11.4 \\
\hline $\mathrm{C} 8$ & VWA & PASSAT WAGON & $\mathrm{I}$ & $\mathrm{F}$ & L4 & 1.8 & G & M & 31.3 & 6.1 & 11.4 \\
\hline \multicolumn{12}{|l|}{ Trucks } \\
\hline T3 & DCC - CHRYSLER & $\begin{array}{l}\text { TOWN \& CNTRY 2WD } \\
\text { FFV }\end{array}$ & D & $\mathrm{F}$ & V6 & 3.3 & FF & A & 20.0 & 7.0 & 9.1 \\
\hline T4 & DCC - DODGE & B2500 WAGON & D & $\mathrm{R}$ & V8 & 5.2 & NG & A & 15.2 & 10.2 & 3.5 \\
\hline T4 & DCC - DODGE & B2500 VAN & D & $\mathrm{R}$ & V8 & 5.2 & $N G$ & A & 15.5 & 10.0 & 3.5 \\
\hline
\end{tabular}




\begin{tabular}{|c|c|c|c|c|c|c|c|c|c|c|c|}
\hline $\begin{array}{l}\text { Type-Size } \\
\text { Class }\end{array}$ & Manufacturer/Division & Name & $\begin{array}{l}\text { Dom I } \\
\text { Imp }\end{array}$ & DR & Cyl & Disp & Fuel & Trans & MPG & GHG & $\begin{array}{l}\text { Oil } \\
\text { Use }\end{array}$ \\
\hline T3 & DCC - DODGE & CARAVAN 2WD FFV & $\mathrm{D}$ & $\mathrm{F}$ & V6 & 3.3 & $\mathrm{FF}$ & $A$ & 19.9 & 7.1 & 9.2 \\
\hline T3 & DCC - DODGE & CARAVAN 2WD & $\mathrm{D}$ & $\mathrm{F}$ & $\mathrm{L} 4$ & 2.4 & G & $A$ & 26.0 & 7.3 & 13.7 \\
\hline T3 & DCC - PLYMOUTH & $\begin{array}{l}\text { CARAVAN/VOYAGER } \\
2 W D\end{array}$ & $\mathrm{D}$ & $\mathrm{F}$ & L4 & 2.4 & G & $A$ & 25.9 & 7.3 & 13.8 \\
\hline T3 & DCC - PLYMOUTH & $\begin{array}{l}\text { CARAVAN/VOYAGER } \\
\text { 2WD }\end{array}$ & $\mathrm{D}$ & $\mathrm{F}$ & V6 & 3.3 & $\mathrm{FF}$ & A & 19.9 & 7.1 & 9.2 \\
\hline T1 & FMC - FORD & RANGER 4X2 EV & $\mathrm{D}$ & $\mathrm{R}$ & E0 & 2.5 & EL & A & 29.7 & 5.2 & 1.8 \\
\hline T2 & FMC - FORD & F150 4X2 NGV & $\mathrm{D}$ & $\mathrm{R}$ & V8 & 5.4 & NG & $A$ & 15.8 & 9.9 & 3.4 \\
\hline $\mathrm{T} 4$ & FMC - FORD & E250 VAN NGV & $\mathrm{D}$ & $\mathrm{R}$ & V8 & 5.4 & NG & $A$ & 15.5 & 10.1 & 3.5 \\
\hline T1 & FMC - FORD & RANGER 4X2 FFV & $\mathrm{D}$ & $\mathrm{R}$ & V6 & 3.0 & $\mathrm{FF}$ & $\mathrm{M}$ & 19.7 & 7.1 & 9.2 \\
\hline T1 & FMC - FORD & RANGER 4X4 FFV & $\mathrm{D}$ & 4 & V6 & 3.0 & $\mathrm{FF}$ & M & 19.2 & 7.3 & 9.5 \\
\hline T1 & FMC - FORD & RANGER 4X2 FFV & $\mathrm{D}$ & $\mathrm{R}$ & V6 & 3.0 & $\mathrm{FF}$ & A & 18.5 & 7.6 & 9.7 \\
\hline T1 & FMC - FORD & RANGER 4X4 FFV & $\mathrm{D}$ & 4 & V6 & 3.0 & $\mathrm{FF}$ & A & 17.7 & 8.0 & 10.4 \\
\hline T1 & FMC - FORD & RANGER 4X2 & $\mathrm{D}$ & $\mathrm{R}$ & $\mathrm{L} 4$ & 2.5 & G & M & 28.3 & 6.7 & 12.6 \\
\hline T1 & GMC - CHEVROLET & S10 P/U 2WD & $\mathrm{D}$ & $\mathrm{R}$ & $\mathrm{L} 4$ & 2.2 & $\mathrm{FF}$ & M & 23.9 & 5.9 & 7.6 \\
\hline T1 & GMC - CHEVROLET & S10 P/U 2WD & $\mathrm{D}$ & $\mathrm{R}$ & $\mathrm{L} 4$ & 2.2 & $\mathrm{FF}$ & $A$ & 21.2 & 6.7 & 8.7 \\
\hline T1 & GMC - CHEVROLET & S10 P/U 2WD & $\mathrm{D}$ & $\mathrm{R}$ & $\mathrm{L} 4$ & 2.2 & G & M & 29.6 & 6.5 & 12.1 \\
\hline T1 & GMC & SONOMA 2WD & I & $\mathrm{R}$ & $\mathrm{L} 4$ & 2.2 & $\mathrm{FF}$ & M & 23.9 & 5.9 & 7.6 \\
\hline T1 & GMC & SONOMA 2WD & I & $\mathrm{R}$ & $\mathrm{L} 4$ & 2.2 & $\mathrm{FF}$ & A & 21.2 & 6.7 & 8.7 \\
\hline T1 & GMC & SONOMA 2WD & I & $\mathrm{R}$ & L4 & 2.2 & G & M & 29.6 & 6.5 & 12.1 \\
\hline T3 & GMC - OLDSMOBILE & SILHOUETTE 2WD & $\mathrm{D}$ & $\mathrm{F}$ & V6 & 3.4 & G & $A$ & 25.0 & 7.6 & 14.3 \\
\hline T3 & HONDA & ODYSSEY & I & $F$ & V6 & 3.5 & G & $A$ & 24.0 & 7.9 & 14.9 \\
\hline T1 & ISUZU & HOMBRE P/U 2WD & I & $\mathrm{R}$ & $\mathrm{L} 4$ & 2.2 & $\mathrm{FF}$ & $M$ & 23.9 & 5.9 & 7.6 \\
\hline T1 & ISUZU & HOMBRE P/U 2WD & I & $\mathrm{R}$ & $\mathrm{L} 4$ & 2.2 & $\mathrm{FF}$ & $A$ & 21.2 & 6.7 & 8.7 \\
\hline T1 & TKM - MAZDA & MAZDA 4X2 FFV & 1 & $\mathrm{R}$ & V6 & 3.0 & $\mathrm{FF}$ & M & 19.7 & 7.1 & 9.2 \\
\hline T1 & TKM - MAZDA & MAZDA 4X4 FFV & 1 & 4 & V6 & 3.0 & $\mathrm{FF}$ & M & 19.2 & 7.3 & 9.5 \\
\hline T1 & TKM - MAZDA & MAZDA 4X2 FFV & I & $\mathrm{R}$ & V6 & 3.0 & $\mathrm{FF}$ & $A$ & 18.5 & 7.6 & 9.7 \\
\hline T1 & TKM - MAZDA & MAZDA 4X4 FFV & I & 4 & V6 & 3.0 & $\mathrm{FF}$ & A & 17.7 & 8.0 & 10.4 \\
\hline T1 & TKM - MAZDA & MAZDA 4X2 & I & $\mathrm{R}$ & $\mathrm{L} 4$ & 2.5 & G & M & 28.3 & 6.7 & 12.6 \\
\hline
\end{tabular}


Energy Star Method 14 Vehicle List

Top $20 \%$ of vehicles by GHG and oil use per footprint [normalized]

\begin{tabular}{|c|c|c|c|c|c|c|c|c|c|c|c|}
\hline $\begin{array}{l}\text { Type-Size } \\
\text { Class }\end{array}$ & Manufacturer/Division & Name & $\begin{array}{l}\text { Dom I } \\
\text { Imp }\end{array}$ & DR & Cyl & Disp & Fuel & Trans & MPG & GHG & $\begin{array}{c}\text { Oil } \\
\text { Use }\end{array}$ \\
\hline \multicolumn{12}{|l|}{ Cars } \\
\hline $\mathrm{C} 4$ & DAEWOO & LANOS- 3Dr & I & $\mathrm{F}$ & L4 & 1.5 & G & M & 34.8 & 5.5 & 10.3 \\
\hline $\mathrm{C} 4$ & DAEWOO & LANOS-3Dr & I & $\mathrm{F}$ & L4 & 1.6 & G & M & 34.6 & 5.5 & 10.3 \\
\hline $\mathrm{C} 4$ & DAEWOO & LANOS- 3Dr & I & $\mathrm{F}$ & L4 & 1.5 & G & A & 32.9 & 5.8 & 10.8 \\
\hline $\mathrm{C} 4$ & DAEWOO & LANOS-4Dr & I & $\mathrm{F}$ & L4 & 1.6 & G & M & 31.8 & 6.0 & 11.2 \\
\hline $\mathrm{C} 4$ & DAEWOO & LANOS-3Dr & I & $\mathrm{F}$ & L4 & 1.6 & G & A & 31.2 & 6.1 & 11.4 \\
\hline $\mathrm{C} 4$ & DAEWOO & NUBIRA & I & $\mathrm{F}$ & L4 & 2.0 & G & M & 30.1 & 6.3 & 11.8 \\
\hline $\mathrm{C7}$ & DAEWOO & NUBIRA WAGON & I & $\mathrm{F}$ & L4 & 2.0 & G & $M$ & 30.1 & 6.3 & 11.8 \\
\hline $\mathrm{C} 7$ & DAEWOO & NUBIRA WAGON & I & $\mathrm{F}$ & L4 & 4.0 & G & $M$ & 30.1 & 6.3 & 11.8 \\
\hline $\mathrm{C} 4$ & DAEWOO & LANOS-4Dr & I & $\mathrm{F}$ & L4 & 1.6 & G & $A$ & 29.7 & 6.4 & 12.0 \\
\hline $\mathrm{C} 4$ & DAEWOO & NUBIRA & 1 & $\mathrm{~F}$ & L4 & 2.0 & G & A & 29.5 & 6.5 & 12.1 \\
\hline $\mathrm{C} 4$ & DAEWOO & NUBIRA & 1 & $\mathrm{~F}$ & L4 & 2.0 & G & M & 30.1 & 6.3 & 11.8 \\
\hline $\mathrm{C} 4$ & DAEWOO & NUBIRA & 1 & $\mathrm{~F}$ & L4 & 2.0 & G & A & 29.5 & 6.5 & 12.1 \\
\hline $\mathrm{C} 3$ & DCC - CHRYSLER & NEON & $\mathrm{D}$ & $\mathrm{F}$ & L4 & 2.0 & G & M & 36.1 & 5.3 & 9.9 \\
\hline $\mathrm{C} 4$ & DCC - CHRYSLER & CIRRUS & $\mathrm{D}$ & $\mathrm{F}$ & L4 & 2.0 & G & M & 34.3 & 5.6 & 10.4 \\
\hline $\mathrm{C} 3$ & DCC - CHRYSLER & NEON & $\mathrm{D}$ & $\mathrm{F}$ & L4 & 2.0 & G & A & 31.9 & 6.0 & 11.2 \\
\hline $\mathrm{C} 3$ & DCC - DODGE & NEON & $\mathrm{D}$ & $\mathrm{F}$ & L4 & 2.0 & G & M & 36.1 & 5.3 & 9.9 \\
\hline C3 & DCC - DODGE & NEON & $\mathrm{D}$ & $\mathrm{F}$ & L4 & 2.0 & G & A & 31.9 & 6.0 & 11.2 \\
\hline C3 & DCC - PLYMOUTH & NEON & $\mathrm{D}$ & $\mathrm{F}$ & L4 & 2.0 & G & M & 36.1 & 5.3 & 9.9 \\
\hline $\mathrm{C} 4$ & DCC - PLYMOUTH & BREEZE & $\mathrm{D}$ & $\mathrm{F}$ & L4 & 2.0 & G & M & 35.4 & 5.4 & 10.1 \\
\hline C3 & DCC - PLYMOUTH & NEON & $\mathrm{D}$ & $\mathrm{F}$ & L4 & 2.0 & G & A & 31.9 & 6.0 & 11.2 \\
\hline C5 & FMC - FORD & CROWN VICTORIA NG & $\mathrm{D}$ & $\mathrm{R}$ & V8 & 4.6 & NG & $A$ & 20.7 & 7.6 & 2.6 \\
\hline $\mathrm{C} 5$ & FMC - FORD & TAURUS FFV & $\mathrm{D}$ & $\mathrm{F}$ & V6 & 3.0 & $\mathrm{FF}$ & A & 24.6 & 5.9 & 7.8 \\
\hline $\mathrm{C} 8$ & FMC - FORD & TAURUS WAGON FFV & $\mathrm{D}$ & $\mathrm{F}$ & V6 & 3.0 & $\mathrm{FF}$ & $A$ & 23.3 & 6.2 & 8.3 \\
\hline $\mathrm{C} 7$ & FMC - FORD & FOCUS WAGON & $\mathrm{D}$ & $\mathrm{F}$ & L4 & 2.0 & G & M & 36.2 & 5.3 & 9.9 \\
\hline C3 & FMC - FORD & FOCUS & $\mathrm{D}$ & $\mathrm{F}$ & L4 & 2.0 & G & M & 35.7 & 5.4 & 10.0 \\
\hline C3 & FMC - FORD & ESCORT & $\mathrm{D}$ & $\mathrm{F}$ & L4 & 2.0 & G & M & 35.7 & 5.4 & 10.0 \\
\hline C3 & FMC - FORD & FOCUS & $\mathrm{D}$ & $\mathrm{F}$ & L4 & 2.0 & G & A & 33.9 & 5.7 & 10.5 \\
\hline $\mathrm{C} 7$ & FMC - FORD & FOCUS WAGON & $\mathrm{D}$ & $\mathrm{F}$ & L4 & 2.0 & G & A & 32.6 & 5.9 & 11.0 \\
\hline $\mathrm{C} 3$ & FMC - FORD & ESCORT & $\mathrm{D}$ & $\mathrm{F}$ & L4 & 2.0 & G & $A$ & 33.7 & 5.7 & 10.6 \\
\hline $\mathrm{C} 2$ & FMC - FORD & ESCORT ZX2 & $\mathrm{D}$ & $\mathrm{F}$ & L4 & 2.0 & G & M & 33.0 & 5.8 & 10.8 \\
\hline C3 & FMC - FORD & FOCUS 3D & $\mathrm{D}$ & $\mathrm{F}$ & L4 & 2.0 & G & M & 33.0 & 5.8 & 10.8 \\
\hline C3 & FMC - FORD & FOCUS 3D & $\mathrm{D}$ & $\mathrm{F}$ & L4 & 2.0 & G & A & 32.4 & 5.9 & 11.0 \\
\hline $\mathrm{C} 2$ & FMC - FORD & ESCORT ZX2 & $\mathrm{D}$ & $\mathrm{F}$ & L4 & 2.0 & G & A & 33.1 & 5.8 & 10.8 \\
\hline $\mathrm{C} 7$ & FMC - FORD & FOCUS WAGON & $\mathrm{D}$ & $\mathrm{F}$ & L4 & 2.0 & G & A & 32.6 & 5.9 & 11.0 \\
\hline C3 & FMC - FORD & CONTOUR & $\mathrm{D}$ & $\mathrm{F}$ & L4 & 2.0 & G & M & 32.6 & 5.9 & 11.0 \\
\hline $\mathrm{C} 3$ & FMC - FORD & CONTOUR & $\mathrm{D}$ & $\mathrm{F}$ & L4 & 2.0 & G & A & 30.3 & 6.3 & 11.8 \\
\hline $\mathrm{C} 3$ & FMC - FORD & CONTOUR & $\mathrm{D}$ & $F$ & L4 & 2.0 & G & $A$ & 30.3 & 6.3 & 11.8 \\
\hline C3 & FMC - MAZDA & PROTEGE & I & $\mathrm{F}$ & L4 & 1.8 & G & A & 30.3 & 6.3 & 11.8 \\
\hline C3 & FMC - MAZDA & PROTEGE & 1 & $\mathrm{~F}$ & L4 & 1.6 & G & A & 33.8 & 5.7 & 10.6 \\
\hline $\mathrm{C} 4$ & FMC - MAZDA & 626 & I & $\mathrm{F}$ & L4 & 2.0 & G & $M$ & 33.5 & 5.7 & 10.6 \\
\hline C3 & FMC - MAZDA & PROTEGE & I & $\mathrm{F}$ & L4 & 1.8 & G & M & 32.4 & 5.9 & 11.0 \\
\hline $\mathrm{C} 6$ & FMC - MAZDA & MX-5 MIATA & 1 & $\mathrm{R}$ & L4 & 1.8 & G & $M$ & 31.4 & 6.1 & 11.4 \\
\hline $\mathrm{C} 3$ & FMC - MAZDA & PROTEGE & I & $\mathrm{F}$ & L4 & 1.6 & G & $M$ & 36.5 & 5.3 & 9.8 \\
\hline $\mathrm{C} 6$ & FMC - MAZDA & MX-5 MIATA & 1 & $\mathrm{R}$ & L4 & 1.8 & G & M & 30.2 & 6.3 & 11.8 \\
\hline $\mathrm{C} 6$ & FMC - MAZDA & MX-5 MIATA & 1 & $\mathrm{R}$ & $\mathrm{L} 4$ & 1.8 & G & A & 28.9 & 6.6 & 12.3 \\
\hline
\end{tabular}




\begin{tabular}{|c|c|c|c|c|c|c|c|c|c|c|c|}
\hline $\begin{array}{l}\text { Type-Size } \\
\text { Class } \\
\end{array}$ & Manufacturer/Division & Name & $\begin{array}{c}\text { Dom I } \\
\text { Imp }\end{array}$ & DR & Cyl & Disp & Fuel & Trans & MPG & GHG & $\begin{array}{l}\text { Oil } \\
\text { Use } \\
\end{array}$ \\
\hline C3 & FMC - MERCURY & MYSTIQUE & D & $\mathrm{F}$ & L4 & 2.0 & G & M & 32.4 & 5.9 & 11.0 \\
\hline C3 & FMC - MERCURY & COUGAR & D & $\mathrm{F}$ & L4 & 2.0 & G & M & 32.4 & 5.9 & 11.0 \\
\hline $\mathrm{C7}$ & FUJI - SUBARU & IMPREZA SW & 1 & 4 & $\mathrm{H} 4$ & 2.2 & G & A & 29.4 & 6.5 & 12.1 \\
\hline C2 & GMC - CHEVY & METRO & 1 & $\mathrm{~F}$ & L3 & 1.0 & G & M & 48.9 & 4.0 & 7.3 \\
\hline $\mathrm{C} 2$ & GMC - CHEVY & METRO & I & $\mathrm{F}$ & L4 & 1.3 & G & M & 45.5 & 4.2 & 7.8 \\
\hline C3 & GMC - CHEVY & PRIZM & 1 & $\mathrm{~F}$ & L4 & 1.8 & G & M & 39.6 & 4.9 & 9.0 \\
\hline C3 & GMC - CHEVY & PRIZM & I & $\mathrm{F}$ & L4 & 1.8 & G & A & 37.5 & 5.1 & 9.5 \\
\hline $\mathrm{C} 2$ & GMC - CHEVY & METRO & I & $\mathrm{F}$ & L4 & 1.3 & G & A & 37.6 & 5.1 & 9.5 \\
\hline C3 & GMC - CHEVY & PRIZM & I & $\mathrm{F}$ & L4 & 1.8 & G & A & 34.9 & 5.5 & 10.2 \\
\hline $\mathrm{C} 2$ & GMC - CHEVY & CAVALIER & D & $\mathrm{F}$ & L4 & 2.2 & G & M & 32.1 & 6.0 & 11.1 \\
\hline $\mathrm{C} 2$ & GMC - CHEVY & CAVALIER & D & $\mathrm{F}$ & $\mathrm{L} 4$ & 2.2 & G & A & 30.7 & 6.2 & 11.6 \\
\hline $\mathrm{C} 2$ & GMC - CHEVY & CAVALIER Z24 & D & $\mathrm{F}$ & L4 & 2.4 & G & M & 30.8 & 6.2 & 11.6 \\
\hline C2 & GMC - PONTIAC & SUNFIRE & D & $\mathrm{F}$ & L4 & 2.2 & G & M & 32.1 & 6.0 & 11.1 \\
\hline $\mathrm{C} 2$ & GMC - PONTIAC & SUNFIRE CNVT & D & $\mathrm{F}$ & L4 & 2.2 & G & A & 30.7 & 6.2 & 11.6 \\
\hline C2 & GMC - PONTIAC & SUNFIRE & D & $\mathrm{F}$ & L4 & 2.4 & G & M & 30.8 & 6.2 & 11.6 \\
\hline C3 & GMC - SATURN & SL & D & $\mathrm{F}$ & L4 & 1.9 & G & M & 38.7 & 5.0 & 9.2 \\
\hline C2 & GMC - SATURN & SC & D & $\mathrm{F}$ & L4 & 1.9 & G & M & 35.4 & 5.4 & 10.1 \\
\hline $\mathrm{C} 2$ & GMC - SATURN & $\mathrm{sc}$ & D & $\mathrm{F}$ & L4 & 1.9 & G & M & 38.1 & 5.0 & 9.4 \\
\hline C3 & GMC - SATURN & $\mathrm{SL}$ & D & $\mathrm{F}$ & L4 & 1.9 & G & M & 36.1 & 5.3 & 9.9 \\
\hline C7 & GMC - SATURN & SW & D & $\mathrm{F}$ & L4 & 1.9 & G & M & 36.1 & 5.3 & 9.9 \\
\hline C3 & GMC - SATURN & SL & D & $\mathrm{F}$ & L4 & 1.9 & G & A & 34.4 & 5.6 & 10.4 \\
\hline C2 & GMC - SATURN & sc & D & $\mathrm{F}$ & L4 & 1.9 & G & A & 33.9 & 5.7 & 10.5 \\
\hline $\mathrm{C} 7$ & GMC - SATURN & sW & D & $\mathrm{F}$ & L4 & 1.9 & G & A & 35.4 & 5.4 & 10.1 \\
\hline C2 & GMC - SATURN & sc & D & $\mathrm{F}$ & L4 & 1.9 & G & A & 36.1 & 5.3 & 9.9 \\
\hline C3 & GMC - SATURN & SL & D & $\mathrm{F}$ & L4 & 1.9 & G & A & 36.0 & 5.3 & 9.9 \\
\hline $\mathrm{C} 7$ & GMC - SATURN & sW & D & $\mathrm{F}$ & L4 & 1.9 & G & A & 33.9 & 5.7 & 10.5 \\
\hline C4 & GMC - SATURN & LS & D & $\mathrm{F}$ & L4 & 2.2 & G & M & 31.9 & 6.0 & 11.2 \\
\hline C4 & GMC - SATURN & LS & D & $\mathrm{F}$ & L4 & 2.2 & G & A & 30.5 & 6.3 & 11.7 \\
\hline $\mathrm{C} 2$ & HONDA - ACURA & ACURA INTEGRA & 1 & $\mathrm{~F}$ & L4 & 1.8 & G & M & 32.3 & 5.9 & 11.0 \\
\hline $\mathrm{C} 2$ & HONDA - ACURA & ACURA INTEGRA -R & 1 & $\mathrm{~F}$ & L4 & 1.8 & G & M & 31.9 & 6.0 & 11.2 \\
\hline $\mathrm{C} 2$ & HONDA - ACURA & ACURA INTEGRA & 1 & $\mathrm{~F}$ & L4 & 1.8 & G & A & 30.7 & 6.2 & 11.6 \\
\hline C6 & HONDA & INSIGHT & 1 & $\mathrm{~F}$ & L3 & 1.0 & $\mathrm{G} / \mathrm{EL}$ & M & 76.3 & 2.6 & 4.7 \\
\hline $\mathrm{C} 2$ & HONDA & CIVIC HX & 1 & $\mathrm{~F}$ & L4 & 1.6 & G & M & 44.7 & 4.3 & 8.0 \\
\hline $\mathrm{C} 2$ & HONDA & CIVIC HX & 1 & $\mathrm{~F}$ & L4 & 1.6 & G & A & 41.9 & 4.6 & 8.5 \\
\hline C2 & HONDA & CIVIC dx 4Dr & 1 & $\mathrm{~F}$ & L4 & 1.6 & G & M & 37.2 & 5.2 & 9.6 \\
\hline $\mathrm{C} 2$ & HONDA & CIVIC ex & 1 & $\mathrm{~F}$ & L4 & 1.6 & G & M & 37.2 & 5.2 & 9.6 \\
\hline $\mathrm{C} 2$ & HONDA & CIVIC vp & 1 & $\mathrm{~F}$ & L4 & 1.6 & G & A & 35.5 & 5.4 & 10.1 \\
\hline C2 & HONDA & CIVIC dx 4Dr & 1 & $\mathrm{~F}$ & L4 & 1.6 & G & A & 35.6 & 5.4 & 10.0 \\
\hline C2 & HONDA & CIVIC ex & 1 & $\mathrm{~F}$ & L4 & 1.6 & G & A & 35.5 & 5.4 & 10.1 \\
\hline $\mathrm{C} 2$ & HONDA & CIVIC si & 1 & $\mathrm{~F}$ & L4 & 1.6 & G & M & 32.8 & 5.8 & 10.9 \\
\hline C4 & HONDA & ACCORD IX & I & $\mathrm{F}$ & L4 & 2.3 & G & M & 32.1 & 6.0 & 11.1 \\
\hline C4 & HONDA & ACCORD & 1 & $\mathrm{~F}$ & L4 & 2.3 & G & M & 30.4 & 6.3 & 11.8 \\
\hline C3 & HYUNDAI & ACCENT & I & $\mathrm{F}$ & L4 & 1.5 & G & M & 36.6 & 5.3 & 9.8 \\
\hline C3 & HYUNDAI & ACCENT & 1 & $\mathrm{~F}$ & L4 & 1.5 & G & A & 34.2 & 5.6 & 10.4 \\
\hline C3 & HYUNDAI & ELANTRA & 1 & $\mathrm{~F}$ & L4 & 2.0 & G & M & 32.1 & 6.0 & 11.1 \\
\hline $\mathrm{C} 7$ & HYUNDAI & ELANTRA WAGON & 1 & $\mathrm{~F}$ & L4 & 2.0 & G & M & 31.3 & 6.1 & 11.4 \\
\hline $\mathrm{C} 2$ & HYUNDAI & TIBURON & 1 & $\mathrm{~F}$ & L4 & 2.0 & G & M & 31.1 & 6.1 & 11.5 \\
\hline C3 & $\mathrm{KIA}$ & SEPHIA L4 & 1 & $\mathrm{~F}$ & L4 & 1.8 & G & A & 29.9 & 6.4 & 12.0 \\
\hline C3 & $\mathrm{KIA}$ & SEPHIA M5 & 1 & $\mathrm{~F}$ & L4 & 1.8 & G & M & 29.5 & 6.5 & 12.1 \\
\hline
\end{tabular}




\begin{tabular}{|c|c|c|c|c|c|c|c|c|c|c|c|}
\hline $\begin{array}{l}\text { Type-Size } \\
\text { Class } \\
\end{array}$ & Manufacturer/Division & Name & $\begin{array}{c}\text { Dom I } \\
\text { Imp }\end{array}$ & DR & Cyl & Disp & Fuel & Trans & MPG & GHG & $\begin{array}{l}\text { Oil } \\
\text { Use } \\
\end{array}$ \\
\hline $\mathrm{C} 2$ & MITSUBISHI & MIRAGE 2D & 1 & $\mathrm{~F}$ & $\mathrm{~L} 4$ & 1.5 & G & M & 41.7 & 4.6 & 8.6 \\
\hline $\mathrm{C} 2$ & MITSUBISHI & MIRAGE & I & $\mathrm{F}$ & L4 & 1.8 & G & M & 36.3 & 5.3 & 9.8 \\
\hline C2 & MITSUBISHI & MIRAGE 2D & I & $\mathrm{F}$ & $\mathrm{L} 4$ & 1.5 & G & A & 36.7 & 5.2 & 9.7 \\
\hline C2 & MITSUBISHI & MIRAGE & 1 & $\mathrm{~F}$ & L4 & 1.8 & G & A & 33.2 & 5.8 & 10.8 \\
\hline $\mathrm{C} 2$ & MITSUBISHI & ECLIPSE & I & $\mathrm{F}$ & L4 & 2.4 & G & M & 30.1 & 6.3 & 11.9 \\
\hline $\mathrm{C} 2$ & MITSUBISHI & ECLIPSE & I & $\mathrm{F}$ & L4 & 2.4 & G & A & 30.1 & 6.3 & 11.9 \\
\hline C3 & NISSAN - INFINITY & INFINITI G20 & I & $\mathrm{F}$ & L4 & 2.0 & G & M & 31.0 & 6.2 & 11.5 \\
\hline C3 & NISSAN - INFINITY & INFINITI G20 & 1 & $\mathrm{~F}$ & L4 & 2.0 & G & A & 30.2 & 6.3 & 11.8 \\
\hline C3 & NISSAN & SENTRA & 1 & $\mathrm{~F}$ & L4 & 1.8 & G & M & 34.9 & 5.5 & 10.2 \\
\hline C3 & NISSAN & SENTRA & I & $F$ & $\mathrm{~L} 4$ & 1.8 & G & A & 33.9 & 5.6 & 10.5 \\
\hline C3 & NISSAN & SENTRA & I & $\mathrm{F}$ & L4 & 2.0 & G & M & 30.9 & 6.2 & 11.6 \\
\hline C3 & NISSAN & ALTIMA & I & $\mathrm{F}$ & L4 & 2.4 & G & M & 30.9 & 6.2 & 11.6 \\
\hline C3 & NISSAN & SENTRA & 1 & $\mathrm{~F}$ & L4 & 2.0 & G & A & 30.8 & 6.2 & 11.6 \\
\hline $\mathrm{C} 2$ & SUZUKI & SWIFT & 1 & $\mathrm{~F}$ & L4 & 1.3 & G & M & 45.5 & 4.2 & 7.8 \\
\hline C2 & SUZUKI & ESTEEM & 1 & $\mathrm{~F}$ & L4 & 1.6 & G & M & 38.6 & 5.0 & 9.3 \\
\hline C2 & SUZUKI & SWIFT & I & $\mathrm{F}$ & L4 & 1.3 & G & A & 37.6 & 5.1 & 9.5 \\
\hline C2 & SUZUKI & ESTEEM & 1 & $\mathrm{~F}$ & L4 & 1.8 & G & M & 36.2 & 5.3 & 9.9 \\
\hline $\mathrm{C} 7$ & SUZUKI & ESTEEM WAGON & I & $\mathrm{F}$ & L4 & 1.8 & G & M & 35.1 & 5.5 & 10.2 \\
\hline C2 & SUZUKI & ESTEEM & 1 & $\mathrm{~F}$ & L4 & 1.6 & G & A & 35.0 & 5.5 & 10.2 \\
\hline $\mathrm{C} 7$ & SUZUKI & ESTEEM WAGON & I & $\mathrm{F}$ & L4 & 1.6 & G & A & 33.7 & 5.7 & 10.6 \\
\hline $\mathrm{C} 7$ & SUZUKI & ESTEEM WAGON & 1 & $\mathrm{~F}$ & L4 & 1.8 & G & A & 33.7 & 5.7 & 10.6 \\
\hline C2 & SUZUKI & ESTEEM & 1 & $\mathrm{~F}$ & $\mathrm{~L} 4$ & 1.8 & G & A & 33.7 & 5.7 & 10.6 \\
\hline C4 & TOYOTA & CAMRY CNG & I & $\mathrm{F}$ & L4 & 2.2 & NG & A & 30.2 & 5.4 & 1.8 \\
\hline C3 & TOYOTA & $\mathrm{ECHO}$ & 1 & $\mathrm{~F}$ & L4 & 1.5 & G & M & 43.1 & 4.5 & 8.3 \\
\hline C3 & TOYOTA & COROLLA & I & $\mathrm{F}$ & L4 & 1.8 & G & M & 39.6 & 4.9 & 9.0 \\
\hline C3 & TOYOTA & $\mathrm{ECHO}$ & I & $\mathrm{F}$ & L4 & 1.5 & G & A & 39.4 & 4.9 & 9.1 \\
\hline C3 & TOYOTA & COROLLA & I & $\mathrm{F}$ & L4 & 1.8 & G & A & 37.4 & 5.1 & 9.6 \\
\hline C2 & TOYOTA & CELICA & 1 & $\mathrm{~F}$ & L4 & 1.8 & G & M & 35.2 & 5.4 & 10.1 \\
\hline $\mathrm{C} 2$ & TOYOTA & CELICA & 1 & $\mathrm{~F}$ & L4 & 1.8 & G & A & 34.9 & 5.5 & 10.2 \\
\hline C3 & TOYOTA & COROLLA & 1 & $\mathrm{~F}$ & L4 & 1.8 & G & A & 34.9 & 5.5 & 10.2 \\
\hline $\mathrm{C} 6$ & TOYOTA & MR2 & 1 & $\mathrm{~F}$ & L4 & 1.8 & G & M & 31.8 & 6.0 & 11.2 \\
\hline C3 & TOYOTA & CAMRY SOLARA & 1 & $\mathrm{~F}$ & L4 & 2.2 & G & M & 31.0 & 6.2 & 11.5 \\
\hline C4 & TOYOTA & CAMRY & I & $\mathrm{F}$ & $\mathrm{L} 4$ & 2.2 & G & M & 31.0 & 6.2 & 11.5 \\
\hline $\mathrm{C} 2$ & TOYOTA & CELICA & 1 & $\mathrm{~F}$ & L4 & 1.8 & G & M & 30.8 & 6.2 & 11.6 \\
\hline C2 & TOYOTA & CELICA & 1 & $\mathrm{~F}$ & L4 & 1.8 & G & A & 30.1 & 6.4 & 11.9 \\
\hline C3 & VWA - AUDI & AUDI A4 & I & $\mathrm{F}$ & L4 & 1.8 & G & M & 31.9 & 6.0 & 11.2 \\
\hline C2 & VWA & NEW BEETLE & 1 & $\mathrm{~F}$ & L4 & 1.9 & D & M & 52.7 & 4.0 & 6.8 \\
\hline C3 & VWA & GOLF TDI & 1 & $\mathrm{~F}$ & L4 & 1.9 & D & M & 52.7 & 4.0 & 6.8 \\
\hline C3 & VWA & JETTA & 1 & $\mathrm{~F}$ & L4 & 1.9 & D & M & 52.7 & 4.0 & 6.8 \\
\hline C2 & VWA & NEW BEETLE & I & $\mathrm{F}$ & L4 & 1.9 & D & A & 44.7 & 4.7 & 8.0 \\
\hline C3 & VWA & GOLF TDI & I & $\mathrm{F}$ & L4 & 1.9 & D & A & 44.7 & 4.7 & 8.0 \\
\hline C3 & VWA & JETTA & 1 & $\mathrm{~F}$ & L4 & 1.9 & D & A & 44.7 & 4.7 & 8.0 \\
\hline C2 & VWA & NEW BEETLE & I & $\mathrm{F}$ & L4 & 1.8 & G & M & 32.3 & 5.9 & 11.1 \\
\hline C2 & VWA & CABRIO & 1 & $\mathrm{~F}$ & L4 & 2.0 & G & M & 31.2 & 6.1 & 11.5 \\
\hline $\mathrm{C} 2$ & VWA & NEW BEETLE & I & $\mathrm{F}$ & L4 & 2.0 & G & M & 31.2 & 6.1 & 11.5 \\
\hline C3 & VWA & GTI & 1 & $\mathrm{~F}$ & L4 & 2.0 & G & M & 31.2 & 6.1 & 11.5 \\
\hline C3 & VWA & GTI & 1 & $\mathrm{~F}$ & L4 & 1.8 & G & M & 31.3 & 6.1 & 11.4 \\
\hline C3 & VWA & GOLF & I & $\mathrm{F}$ & L4 & 1.8 & G & M & 31.3 & 6.1 & 11.4 \\
\hline C3 & VWA & JETTA & I & $\mathrm{F}$ & L4 & 1.8 & G & M & 31.3 & 6.1 & 11.4 \\
\hline
\end{tabular}




\begin{tabular}{|c|c|c|c|c|c|c|c|c|c|c|c|}
\hline $\begin{array}{l}\text { Type-Size } \\
\text { Class }\end{array}$ & Manufacturer/Division & Name & $\begin{array}{l}\text { Dom I } \\
\text { Imp }\end{array}$ & DR & Cyl & Disp & Fuel & Trans & MPG & GHG & $\begin{array}{l}\text { Oil } \\
\text { Use }\end{array}$ \\
\hline $\mathrm{C} 3$ & VWA & GOLF & I & $\mathrm{F}$ & $\mathrm{L} 4$ & 2.0 & G & M & 31.2 & 6.1 & 11.5 \\
\hline C3 & VWA & JETTA & I & $\mathrm{F}$ & L4 & 2.0 & G & M & 31.2 & 6.1 & 11.5 \\
\hline C4 & VWA & PASSAT & 1 & $\mathrm{~F}$ & L4 & 1.8 & G & M & 31.3 & 6.1 & 11.4 \\
\hline C8 & VWA & PASSAT WAGON & 1 & $\mathrm{~F}$ & L4 & 1.8 & G & M & 31.3 & 6.1 & 11.4 \\
\hline \multicolumn{12}{|l|}{ Trucks } \\
\hline T3 & DCC - DODGE & CARAVAN 2WD FFV & $\mathrm{D}$ & $\mathrm{F}$ & V6 & 3.3 & $\mathrm{FF}$ & $A$ & 19.9 & 7.1 & 9.2 \\
\hline T3 & DCC - PLYMOUTH & $\begin{array}{l}\text { CARAVAN/VOYAGER } \\
\text { 2WD }\end{array}$ & D & $\mathrm{F}$ & V6 & 3.3 & FF & $A$ & 19.9 & 7.1 & 9.2 \\
\hline T1 & FMC - FORD & RANGER 4X2 EV & $\mathrm{D}$ & $\mathrm{R}$ & E0 & 2.5 & EL & A & 29.7 & 5.2 & 1.8 \\
\hline T1 & FMC - FORD & RANGER 4X2 FFV & $D$ & $\mathrm{R}$ & V6 & 3.0 & $\mathrm{FF}$ & M & 19.7 & 7.1 & 9.2 \\
\hline T1 & FMC - FORD & RANGER 4X4 FFV & $\mathrm{D}$ & 4 & V6 & 3.0 & $\mathrm{FF}$ & M & 19.2 & 7.3 & 9.5 \\
\hline T1 & GMC - CHEVROLET & S10 P/U 2WD & $\mathrm{D}$ & $\mathrm{R}$ & L4 & 2.2 & $\mathrm{FF}$ & M & 23.9 & 5.9 & 7.6 \\
\hline T1 & GMC - CHEVROLET & S10 P/U 2WD & $D$ & $\mathrm{R}$ & L4 & 2.2 & $\mathrm{FF}$ & A & 21.2 & 6.7 & 8.7 \\
\hline $\mathrm{T} 7$ & GMC - CHEVROLET & TRACKER CONV 2WD & D & $\mathrm{R}$ & L4 & 1.6 & G & M & 31.1 & 6.2 & 11.5 \\
\hline T7 & GMC - CHEVROLET & TRACKER CONV 4X4 & D & 4 & L4 & 1.6 & G & M & 30.7 & 6.2 & 11.6 \\
\hline $\mathrm{T} 7$ & GMC - CHEVROLET & TRACKER CONV 2WD & D & $\mathrm{R}$ & L4 & 2.0 & G & A & 29.1 & 6.6 & 12.3 \\
\hline T1 & GMC & SONOMA 2WD & I & $\mathrm{R}$ & L4 & 2.2 & $\mathrm{FF}$ & M & 23.9 & 5.9 & 7.6 \\
\hline $\mathrm{T} 1$ & GMC & SONOMA 2WD & I & $\mathrm{R}$ & L4 & 2.2 & $\mathrm{FF}$ & A & 21.2 & 6.7 & 8.7 \\
\hline T1 & ISUZU & HOMBRE P/U 2WD & 1 & $\mathrm{R}$ & L4 & 2.2 & $\mathrm{FF}$ & M & 23.9 & 5.9 & 7.6 \\
\hline T1 & ISUZU & HOMBRE P/U 2WD & 1 & $\mathrm{R}$ & L4 & 2.2 & $\mathrm{FF}$ & A & 21.2 & 6.7 & 8.7 \\
\hline $\mathrm{T7}$ & SUZUKI & VITARA CONV 2WD & 1 & $\mathrm{R}$ & L4 & 1.6 & G & M & 31.1 & 6.1 & 11.5 \\
\hline $\mathrm{T7}$ & SUZUKI & VITARA CONV 4WD & 1 & 4 & L4 & 1.6 & G & M & 30.7 & 6.2 & 11.6 \\
\hline T1 & TKM - MAZDA & MAZDA 4X2 FFV & 1 & $\mathrm{R}$ & V6 & 3.0 & $\mathrm{FF}$ & M & 19.7 & 7.1 & 9.2 \\
\hline T1 & TKM - MAZDA & MAZDA 4X4 FFV & I & 4 & V6 & 3.0 & $\mathrm{FF}$ & M & 19.2 & 7.3 & 9.5 \\
\hline T7 & TOYOTA & RAV4 2WD & 1 & $\mathrm{~F}$ & L4 & 2.0 & G & M & 30.9 & 6.2 & 11.6 \\
\hline $\mathrm{T} 7$ & TOYOTA & RAV4 2WD & I & $\mathrm{F}$ & L4 & 2.0 & $\mathrm{G}$ & $A$ & 30.5 & 6.3 & 11.7 \\
\hline
\end{tabular}


Energy Star Technology-Based Method Vehicle List

Top $20 \%$ of vehicles by technology use score

\begin{tabular}{|c|c|c|c|c|c|c|c|c|c|c|c|}
\hline $\begin{array}{l}\text { Type-Size } \\
\text { Class } \\
\end{array}$ & Manufacturer/Division & Name & $\begin{array}{l}\text { Doml } \\
\text { Imp }\end{array}$ & DR & Cyl & Disp & Fuel & Trans & MPG & GHG & $\begin{array}{l}\text { Oil } \\
\text { Use }\end{array}$ \\
\hline \multicolumn{12}{|l|}{ Cars } \\
\hline$\overline{\mathrm{C} 3}$ & BMW & $323 \mathrm{iA}$ & $\mathrm{I}$ & $\mathrm{R}$ & L6 & 2.5 & $\mathrm{G}$ & $A$ & 26.0 & 7.3 & 13.7 \\
\hline C3 & BMW & $323 \mathrm{iA}$ & I & $\mathrm{R}$ & L6 & 2.5 & G & A & 25.2 & 7.3 & 13.7 \\
\hline $\mathrm{C} 2$ & BMW & 323iA CONV. & I & $\mathrm{R}$ & L6 & 2.5 & G & A & 23.3 & 8.2 & 15.4 \\
\hline $\mathrm{C7}$ & BMW & 323iA TOURING & 1 & $\mathrm{R}$ & L6 & 2.5 & G & A & 25.2 & 7.5 & 14.2 \\
\hline C5 & BMW & 740iLA & I & $\mathrm{R}$ & V8 & 4.4 & G & A & 22.7 & 8.4 & 15.8 \\
\hline C6 & BMW & M-COUPE & I & $\mathrm{R}$ & L6 & 3.2 & G & M & 25.1 & 7.6 & 14.2 \\
\hline C6 & BMW & Z3 COUPE 2.8 & I & $\mathrm{R}$ & L6 & 2.8 & G & M & 25.1 & 7.6 & 14.2 \\
\hline C6 & BMW & Z3A COUPE 2.8 & I & $\mathrm{R}$ & L6 & 2.8 & $\mathrm{G}$ & $A$ & 25.2 & 7.6 & 14.2 \\
\hline C4 & DAEWOO & LANOS-3Dr & I & $\mathrm{F}$ & L4 & 1.6 & G & M & 34.6 & 5.5 & 10.3 \\
\hline C4 & DAEWOO & LANOS-4Dr & I & $\mathrm{F}$ & L4 & 1.6 & G & M & 31.8 & 6.0 & 11.2 \\
\hline C4 & DAEWOO & LANOS-4Dr & I & $\mathrm{F}$ & L4 & 1.6 & G & A & 29.7 & 6.4 & 12.0 \\
\hline C5 & DAEWOO & LEGANZA & I & $\mathrm{F}$ & L4 & 2.2 & G & M & 27.6 & 6.9 & 12.9 \\
\hline C5 & DAEWOO & LEGANZA & I & $\mathrm{F}$ & L4 & 2.2 & G & M & 26.4 & 6.9 & 12.9 \\
\hline C5 & DAEWOO & LEGANZA & I & $\mathrm{F}$ & L4 & 2.2 & G & A & 26.7 & 7.1 & 13.4 \\
\hline C5 & DAEWOO & LEGANZA & I & $\mathrm{F}$ & L4 & 2.2 & G & A & 25.9 & 7.1 & 13.4 \\
\hline $\mathrm{C} 4$ & DAEWOO & NUBIRA & I & $\mathrm{F}$ & L4 & 2.0 & G & M & 30.1 & 6.3 & 11.8 \\
\hline C4 & DAEWOO & NUBIRA & I & $\mathrm{F}$ & L4 & 2.0 & G & M & 29.3 & 6.3 & 11.8 \\
\hline $\mathrm{C} 4$ & DAEWOO & NUBIRA & I & $\mathrm{F}$ & L4 & 2.0 & G & A & 29.5 & 6.5 & 12.1 \\
\hline $\mathrm{C} 4$ & DAEWOO & NUBIRA & I & $\mathrm{F}$ & L4 & 2.0 & G & A & 29.2 & 6.5 & 12.1 \\
\hline $\mathrm{C} 7$ & DAEWOO & NUBIRA WAGON & I & $\mathrm{F}$ & L4 & 2.0 & G & M & 30.1 & 6.3 & 11.8 \\
\hline $\mathrm{C} 7$ & DAEWOO & NUBIRA WAGON & I & $\mathrm{F}$ & L4 & 2.0 & G & A & 29.5 & 6.5 & 12.1 \\
\hline $\mathrm{C} 4$ & DCC-CHRYSLER & CIRRUS & D & $\mathrm{F}$ & L4 & 2.0 & G & M & 34.3 & 5.6 & 10.4 \\
\hline $\mathrm{C} 4$ & DCC-DODGE & STRATUS & $\mathrm{D}$ & $\mathrm{F}$ & L4 & 2.0 & G & M & 34.3 & 6.7 & 12.6 \\
\hline $\mathrm{C} 4$ & DCC-DODGE & STRATUS & $\mathrm{D}$ & $\mathrm{F}$ & L4 & 2.0 & G & M & 27.5 & 6.7 & 12.6 \\
\hline $\mathrm{C} 4$ & DCC-PLYMOUTH & BREEZE & $\mathrm{D}$ & $\mathrm{F}$ & L4 & 2.0 & G & A & 27.5 & 6.7 & 12.6 \\
\hline $\mathrm{C} 4$ & FMC-MAZDA & 626 & I & $\mathrm{F}$ & L4 & 2.0 & G & M & 33.5 & 5.7 & 10.6 \\
\hline C3 & FMC-MAZDA & MILLENIA S & I & $\mathrm{F}$ & V6 & 2.3 & G & A & 26.3 & 7.3 & 13.8 \\
\hline C3 & FMC-MAZDA & PROTEGE & I & $\mathrm{F}$ & L4 & 1.6 & G & M & 30.3 & 5.3 & 9.8 \\
\hline C3 & FMC-MAZDA & PROTEGE & I & $\mathrm{F}$ & L4 & 1.6 & G & A & 33.8 & 5.7 & 10.6 \\
\hline C3 & FMC-MAZDA & PROTEGE & 1 & $\mathrm{~F}$ & L4 & 1.8 & G & M & 32.4 & 5.9 & 11.0 \\
\hline C3 & FMC-MAZDA & PROTEGE & I & $\mathrm{F}$ & L4 & 1.8 & G & A & 36.4 & 6.3 & 11.8 \\
\hline C3 & FMC-VOLVO & $\mathrm{S} 40$ & I & $\mathrm{F}$ & L4 & 1.9 & G & A & 28.0 & 6.8 & 12.8 \\
\hline C4 & FMC-VOLVO & S70 & I & $\mathrm{F}$ & L5 & 2.4 & G & A & 25.8 & 7.4 & 13.9 \\
\hline C4 & FMC-VOLVO & $\mathrm{S} 70$ & I & $\mathrm{F}$ & L5 & 2.4 & G & A & 27.2 & 7.0 & 13.1 \\
\hline C4 & FMC-VOLVO & S80 T6 & 1 & $\mathrm{~F}$ & L6 & 2.8 & G & A & 23.7 & 8.1 & 15.3 \\
\hline $\mathrm{C} 7$ & FMC-VOLVO & V40 & I & $\mathrm{F}$ & L4 & 1.9 & G & A & 28.0 & 6.8 & 12.8 \\
\hline C8 & FMC-VOLVO & V70 & I & $\mathrm{F}$ & L5 & 2.4 & G & A & 25.8 & 7.4 & 13.8 \\
\hline $\mathrm{C} 8$ & FMC-VOLVO & V70 & I & $\mathrm{F}$ & L5 & 2.4 & G & A & 27.2 & 7.0 & 13.1 \\
\hline C8 & FMC-VOLVO & V70 R AWD & I & 4 & L5 & 2.3 & G & A & 23.6 & 8.0 & 15.0 \\
\hline C5 & GMC-CADILLAC & DEVILLE & D & $\mathrm{F}$ & V8 & 4.6 & G & A & 24.1 & 7.9 & 14.8 \\
\hline $\mathrm{C} 2$ & GMC-CHEVY & METRO & I & $\mathrm{F}$ & L3 & 1.0 & G & M & 50.8 & 4.0 & 7.3 \\
\hline C4 & GMC-SAAB & SAAB 9-3 & 1 & $\mathrm{~F}$ & L4 & 2.0 & G & M & 28.3 & 6.7 & 12.5 \\
\hline C4 & GMC-SAAB & SAAB 9-3 & I & $\mathrm{F}$ & L4 & 2.0 & G & A & 25.7 & 7.4 & 13.9 \\
\hline $\mathrm{C} 2$ & GMC-SAAB & SAAB 9-3 CVT & I & $\mathrm{F}$ & L4 & 2.0 & G & M & 28.3 & 6.7 & 12.5 \\
\hline $\mathrm{C} 2$ & GMC-SAAB & SAAB 9-3 CVT & I & $\mathrm{F}$ & L4 & 2.0 & G & A & 25.7 & 7.4 & 13.9 \\
\hline $\mathrm{C} 4$ & GMC-SAAB & SAAB 9-3 VIGGEN & I & $\mathrm{F}$ & L4 & 2.3 & G & M & 27.5 & 6.9 & 13.0 \\
\hline$\overline{\mathrm{C} 4}$ & GMC-SAAB & SAAB 9-3 VIGGEN & $\mathrm{I}$ & $F$ & L4 & 2.3 & $\mathrm{G}$ & $\mathrm{M}$ & 27.5 & 6.9 & 13.0 \\
\hline
\end{tabular}




\begin{tabular}{|c|c|c|c|c|c|c|c|c|c|c|c|}
\hline $\begin{array}{l}\text { Type-Size } \\
\text { Class }\end{array}$ & Manufacturer/Division & Name & $\begin{array}{l}\text { Dom/ } \\
\text { Imp }\end{array}$ & DR & Cyl & Disp & Fuel & Trans & MPG & GHG & $\begin{array}{c}\text { Oil } \\
\text { Use } \\
\end{array}$ \\
\hline $\mathrm{C} 4$ & GMC-SAAB & SAAB 9-5 & 1 & $\mathrm{~F}$ & $\mathrm{~L} 4$ & 2.3 & $G$ & $M$ & 26.7 & 6.9 & 12.9 \\
\hline C4 & GMC-SAAB & SAAB 9-5 & I & $\mathrm{F}$ & L4 & 2.3 & G & A & 25.3 & 7.2 & 13.5 \\
\hline $\mathrm{C} 8$ & GMC-SAAB & SAAB 9-5 WAGON & I & $\mathrm{F}$ & L4 & 2.3 & G & M & 26.7 & 7.3 & 13.8 \\
\hline $\mathrm{C} 8$ & GMC-SAAB & SAAB 9-5 WAGON & 1 & $\mathrm{~F}$ & L4 & 2.3 & G & A & 25.3 & 7.7 & 14.4 \\
\hline $\mathrm{C} 2$ & GMC-SATURN & SC & D & $\mathrm{F}$ & L4 & 1.9 & G & M & 38.2 & 5.4 & 10.1 \\
\hline $\mathrm{C} 2$ & GMC-SATURN & sc & D & $\mathrm{F}$ & L4 & 1.9 & G & A & 35.4 & 5.7 & 10.5 \\
\hline C3 & GMC-SATURN & SL & D & $\mathrm{F}$ & L4 & 1.9 & G & M & 36.1 & 5.3 & 9.9 \\
\hline $\mathrm{C} 2$ & HONDA-ACURA & ACURA INTEGRA -R & 1 & $\mathrm{~F}$ & L4 & 1.8 & G & M & 31.9 & 6.0 & 11.2 \\
\hline C4 & HONDA & ACCORD & I & $\mathrm{F}$ & L4 & 2.3 & G & M & 30.4 & 6.3 & 11.8 \\
\hline C4 & HONDA & ACCORD & I & $\mathrm{F}$ & L4 & 2.3 & G & A & 28.6 & 6.7 & 12.5 \\
\hline C4 & HONDA & ACCORD Ix & 1 & $\mathrm{~F}$ & L4 & 2.3 & G & M & 32.1 & 6.0 & 11.1 \\
\hline C4 & HONDA & ACCORD Ix & 1 & $\mathrm{~F}$ & L4 & 2.3 & G & A & 29.9 & 6.4 & 11.9 \\
\hline $\mathrm{C} 2$ & HONDA & CIVIC dx 4Dr & I & $\mathrm{F}$ & L4 & 1.6 & G & M & 39.5 & 5.2 & 9.6 \\
\hline $\mathrm{C} 2$ & HONDA & CIVIC dx 4Dr & I & $\mathrm{F}$ & L4 & 1.6 & G & A & 35.8 & 5.4 & 10.0 \\
\hline $\mathrm{C} 2$ & HONDA & CIVIC HX & 1 & $\mathrm{~F}$ & L4 & 1.6 & G & M & 44.7 & 4.3 & 8.0 \\
\hline $\mathrm{C} 2$ & HONDA & CIVIC HX & 1 & $\mathrm{~F}$ & L4 & 1.6 & G & A & 41.9 & 4.6 & 8.5 \\
\hline $\mathrm{C} 2$ & HONDA & CIVIC vp & 1 & $\mathrm{~F}$ & L4 & 1.6 & G & A & 35.9 & 5.4 & 10.1 \\
\hline C3 & HYUNDAI & ACCENT & I & $\mathrm{F}$ & L4 & 1.5 & G & M & 36.5 & 5.3 & 9.8 \\
\hline $\mathrm{C} 7$ & HYUNDAI & ELANTRA WAGON & I & $\mathrm{F}$ & L4 & 2.0 & G & M & 31.3 & 6.1 & 11.4 \\
\hline $\mathrm{C} 2$ & HYUNDAI & TIBURON & I & $\mathrm{F}$ & L4 & 2.0 & G & M & 31.1 & 6.1 & 11.5 \\
\hline $\mathrm{C} 2$ & HYUNDAI & TIBURON & 1 & $\mathrm{~F}$ & L4 & 2.0 & G & A & 28.9 & 6.6 & 12.4 \\
\hline C3 & $\mathrm{KIA}$ & SEPHIA L4 & 1 & $\mathrm{~F}$ & L4 & 1.8 & G & A & 29.9 & 6.4 & 12.0 \\
\hline C3 & $\mathrm{KIA}$ & SEPHIA M5 & I & $\mathrm{F}$ & L4 & 1.8 & G & M & 29.5 & 6.5 & 12.1 \\
\hline $\mathrm{C} 2$ & MITSUBISHI & MIRAGE & I & $\mathrm{F}$ & L4 & 1.8 & G & M & 36.8 & 5.3 & 9.8 \\
\hline $\mathrm{C} 2$ & MITSUBISHI & MIRAGE & I & $\mathrm{F}$ & L4 & 1.8 & G & A & 33.2 & 5.8 & 10.8 \\
\hline $\mathrm{C} 2$ & MITSUBISHI & MIRAGE 2D & I & $\mathrm{F}$ & L4 & 1.5 & G & M & 41.5 & 4.6 & 8.6 \\
\hline $\mathrm{C} 2$ & MITSUBISHI & MIRAGE 2D & 1 & $\mathrm{~F}$ & L4 & 1.5 & G & A & 36.6 & 5.2 & 9.7 \\
\hline C6 & PORSCH & BOXSTER & 1 & $\mathrm{R}$ & $\mathrm{H} 6$ & 2.5 & G & A & 23.5 & 8.1 & 15.2 \\
\hline C6 & PORSCH & BOXSTER S & I & $\mathrm{R}$ & $\mathrm{H} 6$ & 2.5 & G & M & 23.8 & 8.0 & 15.0 \\
\hline C6 & PORSCH & BOXSTER S & I & $\mathrm{R}$ & $\mathrm{H} 6$ & 2.5 & G & A & 22.5 & 8.4 & 15.9 \\
\hline $\mathrm{C} 2$ & SUZUKI & ESTEEM & I & $\mathrm{F}$ & L4 & 1.6 & G & M & 38.6 & 5.0 & 9.3 \\
\hline $\mathrm{C} 2$ & SUZUKI & ESTEEM & 1 & $\mathrm{~F}$ & L4 & 1.6 & G & A & 35.0 & 5.5 & 10.2 \\
\hline $\mathrm{C} 2$ & SUZUKI & ESTEEM & I & $\mathrm{F}$ & L4 & 1.8 & G & M & 36.2 & 5.3 & 9.9 \\
\hline $\mathrm{C} 2$ & SUZUKI & ESTEEM & I & $\mathrm{F}$ & L4 & 1.8 & G & A & 33.3 & 5.7 & 10.6 \\
\hline $\mathrm{C} 7$ & SUZUKI & ESTEEM WAGON & 1 & $\mathrm{~F}$ & L4 & 1.6 & G & A & 33.7 & 5.7 & 10.6 \\
\hline C4 & TOYOTA-LEXUS & GS 300 & 1 & $\mathrm{R}$ & L6 & 3.0 & G & A & 24.9 & 7.7 & 14.4 \\
\hline $\mathrm{C} 2$ & TOYOTA-LEXUS & SC 300/SC 400 & 1 & $\mathrm{R}$ & L6 & 3.0 & G & A & 24.1 & 7.9 & 14.8 \\
\hline $\mathrm{C} 2$ & TOYOTA-LEXUS & SC 400 & 1 & $\mathrm{R}$ & V8 & 4.0 & G & A & 24.3 & 7.8 & 14.7 \\
\hline C4 & TOYOTA & CAMRY & 1 & $\mathrm{~F}$ & L4 & 2.2 & G & M & 31.0 & 6.2 & 11.5 \\
\hline $\mathrm{C} 2$ & TOYOTA & CELICA & 1 & $\mathrm{~F}$ & L4 & 1.8 & G & M & 35.2 & 5.4 & 10.1 \\
\hline $\mathrm{C} 2$ & TOYOTA & CELICA & 1 & $\mathrm{~F}$ & L4 & 1.8 & G & A & 34.9 & 5.5 & 10.2 \\
\hline $\mathrm{C} 2$ & TOYOTA & CELICA & I & $\mathrm{F}$ & L4 & 1.8 & G & M & 30.8 & 6.2 & 11.6 \\
\hline C2 & TOYOTA & CELICA & I & $\mathrm{F}$ & L4 & 1.8 & G & A & 30.1 & 6.4 & 11.9 \\
\hline C3 & TOYOTA & COROLLA & 1 & $\mathrm{~F}$ & L4 & 1.8 & G & A & 34.9 & 5.5 & 10.2 \\
\hline C3 & TOYOTA & COROLLA & 1 & $\mathrm{~F}$ & L4 & 1.8 & G & M & 39.6 & 4.9 & 9.0 \\
\hline C3 & TOYOTA & COROLLA & I & $\mathrm{F}$ & L4 & 1.8 & G & A & 37.4 & 5.1 & 9.6 \\
\hline C3 & TOYOTA & $\mathrm{ECHO}$ & I & $\mathrm{F}$ & L4 & 1.5 & G & M & 43.1 & 4.5 & 8.3 \\
\hline C3 & TOYOTA & $\mathrm{ECHO}$ & I & $\mathrm{F}$ & L4 & 1.5 & G & A & 39.5 & 4.9 & 9.1 \\
\hline C3 & AUDI & AUDI A4 & I & $\mathrm{F}$ & L4 & 1.8 & G & M & 31.9 & 6.0 & 11.2 \\
\hline$\overline{\mathrm{C} 3}$ & AUDI & AUDI A4 & $\mathrm{T}$ & $\mathrm{F}$ & L4 & 1.8 & $G$ & $A$ & 28.0 & 6.8 & 12.8 \\
\hline
\end{tabular}




\begin{tabular}{|c|c|c|c|c|c|c|c|c|c|c|c|}
\hline $\begin{array}{l}\text { Type-Size } \\
\text { Class }\end{array}$ & Manufacturer/Division & Name & $\begin{array}{l}\text { Doml } \\
\text { Imp }\end{array}$ & DR & Cyl & Disp & Fuel & Trans & MPG & GHG & $\begin{array}{l}\text { Oil } \\
\text { Use }\end{array}$ \\
\hline$\overline{\mathrm{C} 3}$ & AUDI & AUDI A4 TT C QUATTRO & 1 & 4 & L4 & 1.8 & $\bar{G}$ & $\bar{M}$ & 27.2 & 7.0 & $\overline{13.1}$ \\
\hline $\mathrm{C} 4$ & VWA & PASSAT & I & $\mathrm{F}$ & L4 & 1.8 & G & A & 27.4 & 7.0 & 13.1 \\
\hline C8 & VWA & PASSAT WAGON & I & $\mathrm{F}$ & L4 & 1.8 & G & A & 27.4 & 7.0 & 13.1 \\
\hline \multicolumn{12}{|l|}{ Trucks } \\
\hline T5 & BMW & $\mathrm{X} 5$ & $\mathrm{I}$ & 4 & V8 & 4.4 & $\mathrm{G}$ & $A$ & 17.5 & 10.8 & $\overline{20.4}$ \\
\hline T4 & DCC-DODGE & B1500 WAGON & $\mathrm{D}$ & $\mathrm{R}$ & V6 & 3.9 & G & $A$ & 17.7 & 10.7 & 20.2 \\
\hline T4 & DCC-DODGE & B1500 WAGON & $\mathrm{D}$ & $\mathrm{R}$ & V8 & 5.2 & G & $A$ & 17.5 & 10.8 & 20.4 \\
\hline T3 & DCC-DODGE & CARAVAN 2WD & $\mathrm{D}$ & $\mathrm{F}$ & L4 & 2.4 & G & $A$ & 26.0 & 7.3 & 13.7 \\
\hline T3 & DCC-DODGE & CARAVAN 2WD & $\mathrm{D}$ & $\mathrm{F}$ & V6 & 3.0 & G & $A$ & 24.0 & 7.9 & 14.9 \\
\hline T3 & DCC-DODGE & CARAVAN 2WD & $\mathrm{D}$ & $\mathrm{F}$ & V6 & 3.3 & G & $A$ & 24.3 & 7.8 & 14.7 \\
\hline T3 & DCC-DODGE & CARAVAN 2WD FFV & $\mathrm{D}$ & $F$ & V6 & 3.3 & $\mathrm{FF}$ & $A$ & 40.4 & 7.1 & 9.2 \\
\hline T1 & DCC-DODGE & DAKOTA 2WD & $\mathrm{D}$ & $\mathrm{R}$ & L4 & 2.5 & G & M & 25.7 & 7.4 & 13.9 \\
\hline T2 & DCC-DODGE & RAM 1500 4WD & $\mathrm{D}$ & 4 & V8 & 5.2 & G & M & 17.0 & 11.8 & 22.4 \\
\hline T2 & DCC-DODGE & RAM 1500 4WD & $\mathrm{D}$ & 4 & V8 & 5.2 & G & $A$ & 16.0 & 11.1 & 21.0 \\
\hline T5 & DCC-JEEP & CHEROKEE 2WD & I & $\mathrm{R}$ & L4 & 2.5 & G & $A$ & 23.7 & 8.0 & 15.1 \\
\hline T5 & DCC-JEEP & CHEROKEE 2WD & I & $\mathrm{R}$ & L4 & 2.5 & G & M & 25.7 & 7.4 & 13.9 \\
\hline T5 & DCC-JEEP & CHEROKEE 4WD & I & 4 & L4 & 2.5 & G & M & 22.2 & 8.5 & 16.1 \\
\hline T5 & DCC-MER-BENZ & ML 320 & 1 & 4 & V6 & 3.2 & G & $A$ & 20.8 & 9.1 & 17.2 \\
\hline T3 & DCC-PLYMOUTH & $\begin{array}{l}\text { CARAVAN/VOYAGER } \\
2 W D\end{array}$ & D & $F$ & L4 & 2.4 & G & $A$ & 26.0 & 7.3 & 13.8 \\
\hline T3 & DCC-PLYMOUTH & $\begin{array}{l}\text { CARAVAN/VOYAGER } \\
\text { 2WD }\end{array}$ & $\mathrm{D}$ & $\mathrm{F}$ & V6 & 3.0 & G & A & 24.0 & 7.9 & 14.9 \\
\hline T3 & DCC-PLYMOUTH & $\begin{array}{l}\text { CARAVAN/VOYAGER } \\
\text { 2WD }\end{array}$ & D & $\mathrm{F}$ & V6 & 3.3 & $\mathrm{FF}$ & $A$ & 24.3 & 7.1 & 9.2 \\
\hline T3 & DCC-PLYMOUTH & $\begin{array}{l}\text { CARAVAN/VOYAGER } \\
2 W D\end{array}$ & $\mathrm{D}$ & $\mathrm{F}$ & V6 & 3.3 & G & $A$ & 40.4 & 7.9 & 14.9 \\
\hline T6 & FMC-FORD & EXPEDITION 4X2 & $\mathrm{D}$ & $\mathrm{R}$ & V8 & 4.6 & G & $A$ & 19.4 & 9.7 & 18.4 \\
\hline T2 & FMC-FORD & $\mathrm{F} 1504 \mathrm{X} 4$ & $\mathrm{D}$ & 4 & V8 & 4.6 & G & $A$ & 18.7 & 10.0 & 19.0 \\
\hline T2 & FMC-FORD & $\mathrm{F} 1504 \mathrm{X} 4$ & $\mathrm{D}$ & 4 & V8 & 4.6 & G & M & 18.5 & 10.3 & 19.5 \\
\hline T2 & GMC-CHEVROLET & C1500 SILVERADO 2WD & $\mathrm{D}$ & $\mathrm{R}$ & V8 & 5.3 & G & $A$ & 18.6 & 10.2 & 19.2 \\
\hline T6 & GMC-CHEVROLET & C1500 TAHOE 2WD & $\mathrm{D}$ & $\mathrm{R}$ & V8 & 4.8 & G & $A$ & 18.5 & 9.7 & 18.3 \\
\hline T2 & GMC-CHEVROLET & C2500 SILVERADO 2WD & $\mathrm{D}$ & $\mathrm{R}$ & V8 & 6.0 & G & $A$ & 16.0 & 11.9 & 22.5 \\
\hline T2 & GMC-CHEVROLET & C2500 SILVERADO 2WD & $\mathrm{D}$ & $\mathrm{R}$ & V8 & 5.3 & G & $A$ & 18.6 & 10.2 & 19.2 \\
\hline T4 & GMC-CHEVROLET & G15/25 CHEV EXPR & $\mathrm{D}$ & $\mathrm{R}$ & V6 & 4.3 & G & $A$ & 18.2 & 10.3 & 19.5 \\
\hline T4 & GMC-CHEVROLET & G15/25 CHEV EXPR & $\mathrm{D}$ & $\mathrm{R}$ & V8 & 5.0 & G & $A$ & 17.9 & 10.5 & 20.0 \\
\hline T4 & GMC-CHEVROLET & G15/25 CHEV EXPR & $\mathrm{D}$ & $\mathrm{R}$ & V8 & 5.7 & G & $A$ & 16.8 & 11.1 & 21.1 \\
\hline T1 & GMC-CHEVROLET & S10 P/U 2WD & $\mathrm{D}$ & $\mathrm{R}$ & L4 & 2.2 & $\mathrm{FF}$ & $A$ & 40.1 & 6.7 & 8.7 \\
\hline T1 & GMC-CHEVROLET & S10 P/U 2WD & $\mathrm{D}$ & $\mathrm{R}$ & L4 & 2.2 & $\mathrm{FF}$ & M & 41.6 & 5.9 & 7.6 \\
\hline T1 & GMC-CHEVROLET & S10 P/U 2WD & $\mathrm{D}$ & $\mathrm{R}$ & L4 & 2.2 & G & $A$ & 26.2 & 7.5 & 14.2 \\
\hline T1 & GMC-CHEVROLET & S10 P/U 2WD & $\mathrm{D}$ & $\mathrm{R}$ & L4 & 2.2 & G & M & 29.4 & 6.5 & 12.1 \\
\hline $\mathrm{T} 7$ & GMC-CHEVROLET & TRACKER CONV 2WD & $\mathrm{D}$ & $\mathrm{R}$ & L4 & 1.6 & G & M & 31.1 & 6.2 & 11.5 \\
\hline $\mathrm{T} 7$ & GMC-CHEVROLET & TRACKER CONV 2WD & $\mathrm{D}$ & $\mathrm{R}$ & L4 & 2.0 & G & $A$ & 28.6 & 6.6 & 12.3 \\
\hline T7 & GMC-CHEVROLET & TRACKER CONV 2WD & $\mathrm{D}$ & $\mathrm{R}$ & L4 & 2.0 & G & M & 28.4 & 6.8 & 12.8 \\
\hline T7 & GMC-CHEVROLET & TRACKER CONV 4X4 & $\mathrm{D}$ & 4 & L4 & 1.6 & G & M & 30.7 & 6.2 & 11.6 \\
\hline $\mathrm{T} 7$ & GMC-CHEVROLET & TRACKER CONV 4X4 & $\mathrm{D}$ & 4 & L4 & 2.0 & G & $A$ & 27.8 & 6.8 & 12.8 \\
\hline $\mathrm{T} 7$ & GMC-CHEVROLET & TRACKER CONV 4X4 & $\mathrm{D}$ & 4 & L4 & 2.0 & G & M & 27.6 & 6.9 & 13.0 \\
\hline T6 & GMC & C1500 YUKON 2WD & I & $\mathrm{R}$ & V8 & 4.8 & G & $A$ & 18.5 & 9.7 & 18.3 \\
\hline T2 & GMC & C2500 SIERRA 2WD & I & $\mathrm{R}$ & V8 & 5.3 & G & $A$ & 18.6 & 10.2 & 19.2 \\
\hline T4 & GMC & G15/25SAVANA(P) & I & $\mathrm{R}$ & V6 & 4.3 & G & $A$ & 18.2 & 10.3 & 19.5 \\
\hline T4 & GMC & G15/25SAVANA(P) & I & $\mathrm{R}$ & V8 & 5.0 & G & $A$ & 17.9 & 10.5 & 19.9 \\
\hline T2 & GMC & K1500 SIERRA 4WD & I & 4 & V8 & 4.8 & G & $A$ & 19.0 & 9.9 & 18.7 \\
\hline$\overline{T 2}$ & GMC & K1500 SIERRA 4WD & $\mathrm{I}$ & 4 & V8 & 4.8 & $\mathrm{G}$ & $\mathrm{M}$ & 19.1 & 9.8 & $\overline{18.5}$ \\
\hline T1 & GMC & SONOMA 2WD & I & $\mathrm{R}$ & L4 & 2.2 & G & M & 29.4 & 6.5 & 12.1 \\
\hline
\end{tabular}




\begin{tabular}{|c|c|c|c|c|c|c|c|c|c|c|c|}
\hline $\begin{array}{l}\text { Type-Size } \\
\text { Class }\end{array}$ & Manufacturer/Division & Name & $\begin{array}{l}\text { Dom/ } \\
\text { Imp }\end{array}$ & DR & Cyl & Disp & Fuel & Trans & MPG & GHG & $\begin{array}{l}\text { Oil } \\
\text { Use }\end{array}$ \\
\hline T5 & ISUZU & AMIGO 2WD & $\mathrm{I}$ & $\mathrm{R}$ & L4 & 2.2 & $\bar{G}$ & $\mathrm{M}$ & 24.7 & 7.6 & $\overline{14.4}$ \\
\hline T1 & ISUZU & HOMBRE P/U 2WD & I & $\mathrm{R}$ & L4 & 2.2 & G & M & 27.5 & 6.7 & 12.6 \\
\hline T1 & ISUZU & HOMBRE P/U 2WD & I & $\mathrm{R}$ & L4 & 2.2 & G & $A$ & 23.8 & 7.7 & 14.5 \\
\hline T1 & ISUZU & HOMBRE P/U 2WD & I & $\mathrm{R}$ & L4 & 2.2 & FF & $A$ & 41.4 & 6.7 & 8.7 \\
\hline T1 & ISUZU & HOMBRE P/U 2WD & I & $\mathrm{R}$ & L4 & 2.2 & FF & M & 43.3 & 5.9 & 7.6 \\
\hline T5 & ISUZU & RODEO 2WD & I & $\mathrm{R}$ & L4 & 2.2 & $G$ & M & 24.9 & 7.6 & 14.4 \\
\hline T5 & MITSUBISHI & $\begin{array}{l}\text { MONTERO } \\
\text { SPORT/NATIVA }\end{array}$ & I & $\mathrm{R}$ & L4 & 2.3 & G & $A$ & 24.2 & 7.8 & 14.7 \\
\hline T5 & MITSUBISHI & $\begin{array}{l}\text { MONTERO } \\
\text { SPORT/NATIVA }\end{array}$ & I & $\mathrm{R}$ & L4 & 2.3 & G & M & 26.5 & 7.2 & 13.5 \\
\hline T1 & NISSAN & FRONTIER TRUCK 2WD & I & $\mathrm{R}$ & L4 & 2.4 & G & $A$ & 24.8 & 7.6 & 14.3 \\
\hline T1 & NISSAN & FRONTIER TRUCK 2WD & I & $\mathrm{R}$ & L4 & 2.4 & G & M & 27.6 & 6.8 & 12.7 \\
\hline T5 & NISSAN & XTERRA 2WD & I & $\mathrm{R}$ & L4 & 2.4 & G & M & 23.9 & 7.9 & 14.8 \\
\hline T5 & SUBARU & FORESTER & I & 4 & $\mathrm{HO} 4$ & 2.5 & G & $A$ & 27.8 & 6.8 & 12.8 \\
\hline T5 & SUBARU & FORESTER & I & 4 & $\mathrm{HO} 4$ & 2.5 & G & M & 27.9 & 6.8 & 12.8 \\
\hline $\mathrm{T} 7$ & SUZUKI & VITARA CONV 2WD & I & $\mathrm{R}$ & L4 & 1.6 & G & M & 31.1 & 6.1 & 11.5 \\
\hline $\mathrm{T} 7$ & SUZUKI & VITARA CONV 4WD & I & 4 & L4 & 1.6 & G & M & 30.7 & 6.2 & 11.6 \\
\hline T5 & TOYOTA-LEXUS & LX 470 & I & 4 & V8 & 4.7 & G & A & 17.1 & 11.0 & 20.9 \\
\hline $\mathrm{T7}$ & TOYOTA-LEXUS & RX 300 2WD & I & $\mathrm{F}$ & V6 & 3.0 & G & A & 24.2 & 7.8 & 14.8 \\
\hline $\mathrm{T} 7$ & TOYOTA-LEXUS & RX 300 4WD & I & 4 & V6 & 3.0 & G & $A$ & 23.4 & 8.1 & 15.3 \\
\hline T5 & TOYOTA & 4RUNNER 2WD & I & $\mathrm{R}$ & L4 & 2.7 & G & $A$ & 25.0 & 7.6 & 14.3 \\
\hline T5 & TOYOTA & 4RUNNER 2WD & I & $\mathrm{R}$ & L4 & 2.7 & G & M & 23.8 & 8.0 & 15.0 \\
\hline T5 & TOYOTA & 4RUNNER 4WD & I & 4 & L4 & 2.7 & G & $A$ & 23.2 & 8.2 & 15.3 \\
\hline T5 & TOYOTA & 4RUNNER 4WD & I & 4 & L4 & 2.7 & G & M & 22.0 & 8.7 & 16.3 \\
\hline T1 & TOYOTA & TOYOTA TACOMA 2WD & I & $\mathrm{R}$ & L4 & 2.4 & G & $A$ & 26.2 & 7.2 & 13.6 \\
\hline $\mathrm{T} 1$ & TOYOTA & TOYOTA TACOMA 2WD & I & $\mathrm{R}$ & L4 & 2.4 & G & M & 27.2 & 7.0 & 13.1 \\
\hline
\end{tabular}

